\title{
Estimating Chronic wasting disease infectivity in cell culture
}

In partial fulfilment of the requirements for the degree

"Doctor rerum naturalium (Dr. rer. nat.)“
in the Molecular Medicine Study Program
at the Georg-August University Göttingen

submitted by

Ann-Christin Schmädicke

born in Potsdam, Germany

Göttingen, October 2011 
Für meinen Vater 


\section{Thesis Committee}

\section{Supervisor}

Prof. Dr. Gerhard Hunsmann

Head (retired), Department of Virology and Immunology

German Primate Center

Leibniz-Institute for primate research

Kellnerweg 4

D-37077 Göttingen

and

Head (retired), Department of Virology

University Medical Center

Kreuzbergring 57

D-37075 Göttingen

\section{Second member of the thesis committee}

Prof. Dr. Thomas Bayer

Head, Division of Molecular Psychiatry

University Medical Center

Georg-August University

Von-Siebold-Straße 5

D-37075 Göttingen

\section{Third member of the thesis committee}

Prof. Dr. Uwe Groß

Head, Institute for Medical Microbiology

University Medical Center

Georg-August University

Kreuzbergring 57

D-37075 Göttingen 


\section{Affidavit}

I hereby declare that my doctoral thesis entitled "Estimating Chronic wasting disease infectivity in cell culture" has been written independently with no other aids or sources than those quoted.

Ann-Christin Schmädicke

October 2011

Göttingen, Germany 


\section{Related publications}

Within this thesis, the following publication has been released. I am grateful to all coauthors for the inspiring collaboration.

Greenwood AD, Vincendeau M, Schmädicke AC, Montag J, Seifarth W and Motzkus D Molecular Neurodegeneration published 2011, June 23

- "Bovine spongiform encephalopathy infection alters endogenous retrovirus expression in distinct brain regions of cynomolgus macaques (Macaca fascicularis)."

Parts of this thesis have been presented at international conferences. I want to thank all people who contributed to these studies.

- Poster "Comprehensive risk assessment of CWD transmission to humans using non-human primates"

PrP Canada 2009, Edmonton March 2009

- Poster "Estimation of Chronic Wasting disease (CWD) infectivity in cell culture"

Prion 2009, Thessaloniki/Chalkidiki September 2009

- Poster "A cell culture based model to simulate PrPSc infection in nonhuman primates"

PrP Canada 2010, Ottawa March 2010

- Poster "Estimating Chronic Wasting disease infectivity in cell culture" Summer School "From Pluripotency to Senescence: Molecular Mechanisms of Development, Disease and Ageing" Spetses Island, August 2010

- Poster "Differential gene expression in BSE-inoculated macaques"

Prion 2010, Salzburg September 2010

- Poster "A cell culture based model to simulate PrPSc infection in nonhuman primates"

PrP Canada 2011, Montreal May 2011

- Group meeting “Update on 'Comprehensive risk assessment of Chronic wasting disease transmission to humans using non-human primates' " PrP Canada 2011, Montreal May 2011 


\section{Acknowledgment}

This work was conducted in the Unit of Infection Models (formally part of the Dept. of Virology and Immunology), German Primate Center GmbH, Göttingen, Germany. I would like to thank Prof. Dr. Gerhard Hunsmann as my supervisor for giving me the opportunity to conduct this work. I also want to acknowledge my thesis committee members Prof. Dr. Thomas Bayer and Prof. Dr. Uwe Groß for their interest in my project and intellectual support. I would like to thank Dr. Christiane Stahl-Hennig, head of the unit of infection models, for the opportunity to complete my work within her group.

I express my gratitude to Dr. Dirk Motzkus. Thank you for your constant excellent supervision and your scientific guidance.

I sincerely thank our collaborators: Prof. Dr. Hermann Schätzl and Dr. Sabine Gilch for support in prion cell culture, Dr. Walter Schulz-Schaeffer, Dr. Wiebke Wemheuer and Johanna Breyer for support in neuropathological questions and Western blot, Dr. Michael Beekes and Dr. Katja Wagenführ for support in ultracentifugation techniques and Dr. Ina Vorberg for support in retroviral transduction techniques, the supply of cell lines and fruitful scientific discussions. Additionally, I would like to thank Prof. Dr. Jürgen Richt for providing the animal tissue used for infection experiments, Prof. Dr. T. Onodera for providing the HpL3-4 cell line, and Prof. Dr. Stefanie Czub for the coordination of the whole CWD risk assessment study.

I also would like to thank Dr. Tina Schultheiß and Dr. Katharina Töpfer for flow cytometry applications, Wiebke Ibing for support in cloning techniques, Dr. Judith Montag for education under BSL-3 conditions and Katrin Donde for help during infection experiments. My special thanks go to Sabine Borchert, Georgia Hesse and Mareike Curth for excellent technical assistance.

I am very grateful to all members of the Units of Infection Models and Infection Biology for their help and the enjoyable atmosphere in the lab.

I want to acknowledge the Molecular Medicine PhD program for administrative support and last but not least the Alberta Prion Research Institute and the Lower Saxony Ministry of Science and Culture for funding this project. 


\section{Content}

I List of Figures ........................................................................................

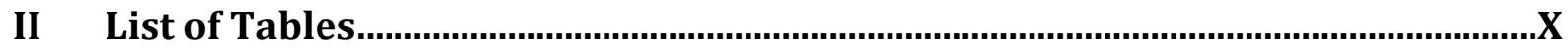

III List of Abbreviations............................................................................................

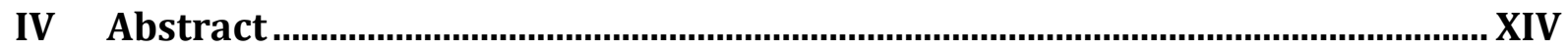

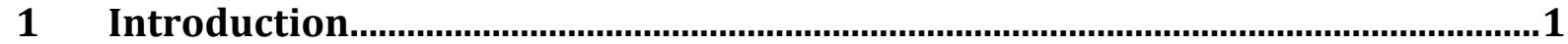

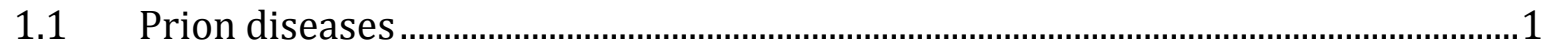

1.2 The causative agent of prion diseases ....…………………………………………...

1.3 Prion diseases of animals ........................................................................................6

1.4 Prion diseases in humans......................................................................................... 10

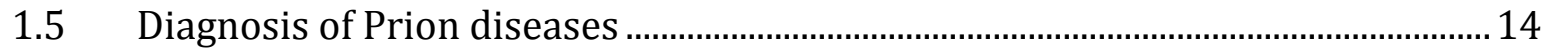

1.6 Animal models in Prion research ........................................................................... 17

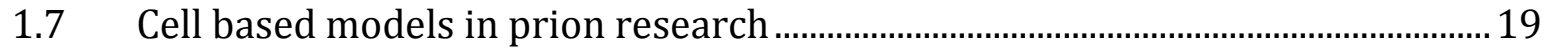

$1.8 \quad$ Outline of the thesis..............................................................................................22

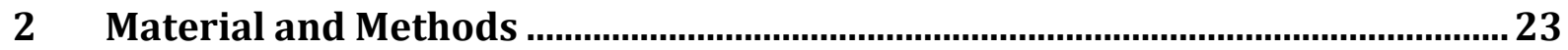

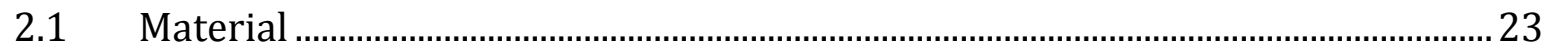

2.1.1 Consumables and Equipment...............................................................................2

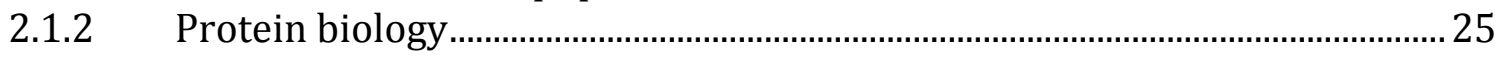

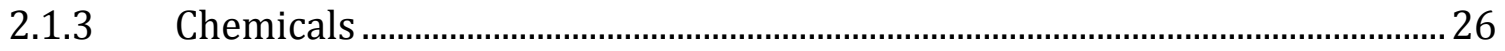

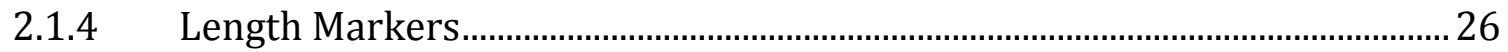

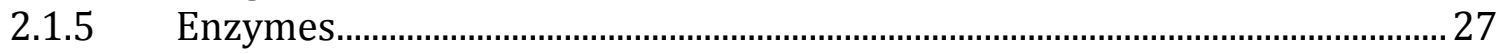

2.1.6 Reaction components and commercial kits....................................................2.

2.1.7 Antibodies for protein detection...........................................................................2

2.1.8 Oligonucleotides for PCR ……………………………………………………….. 31

2.1.9 Plasmids...........................................................................................................

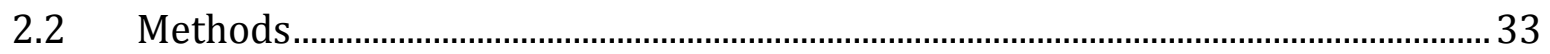

2.2.1 Cell biology ………………………………………………………………………..... 33

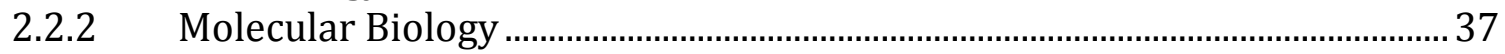

2.2.3 Protein biochemistry .......................................................................................... 46

2.2.4 Computational biology..................................................................................... 50

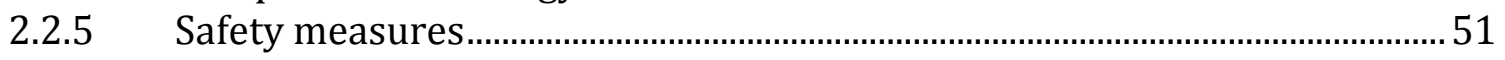

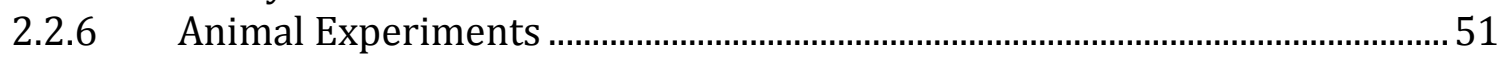

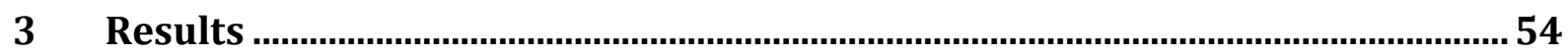

3.1 Generation of retroviral expression plasmids encoding the prion protein of

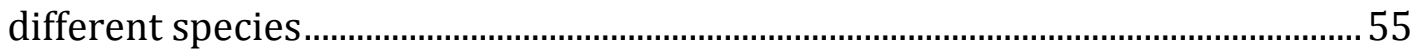

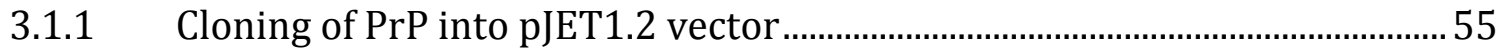

3.1.2 Cloning of PrP cDNAs into retroviral expression vector (pBABE) ................57

3.2 Generation of cell lines overexpressing the prion protein of different species 58

3.2.1 Choice of cell lines suitable to examine the zoonotic potential of prioninfectious agents................................................................................................... 58

3.2.2 Generation and transduction of replication defective retroviral particles

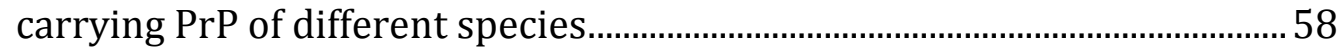

3.2.3 Estimating the transduction efficiency of replication-defective retroviral particles carrying EGFP transcript..

3.2.4 Acute toxicity test of puromycin in HpL3-4 cells and SH-SY5Y ......................61

3.2.5 Generation of transgenic PrP HpL3-4 and SH-SY5Y cell lines........................64 
3.3 Molecular and biochemical characterisation of PrP transgenic HpL3-4 and SH-

SY5Y cell lines

3.3.1 Quantification of transgene integration sites into the host cell genome of HpL3-4 and SH-SY5Y cells

3.3.2 Expression of transgenic PrP in HpL3-4 and SH-SY5Y cells.............................68

3.3.3 Expression of prion protein in transgenic HpL3-4 and SH-SY5Y cell lines70

3.3.4 Surface presentation of transgenic PrP in HpL3-4 and SH-SY5Y cells........ 73

3.3.5 Summary of transgenic PrP cell line characterisation ........................................... 82

3.4 Infection of transgenic cell lines with prion-infectious agents...............................83

3.4.1 Detection of cell culture-adapted 22L Scrapie PrPres in permanently

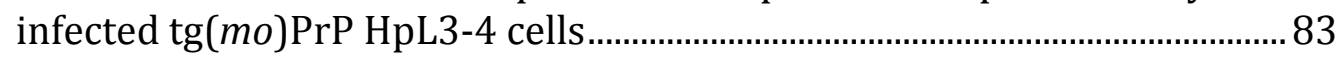

3.4.2 Influence of fetal calf serum on PrPres propagation in $\operatorname{tg}(m o) \operatorname{PrP} H p L 3-4$ cells

3.4.3 Infection conditions for cell culture adapted 22L Scrapie in $\operatorname{tg}(m o) \operatorname{PrP}$

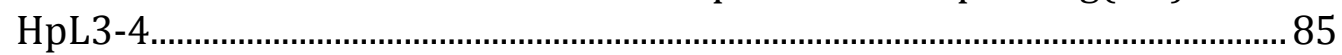

3.4.4 Inoculation of transgenic PrP expressing HpL3-4 and SH-SY5Y cell lines with cell culture adapted $22 \mathrm{~L}$

3.4.5 Inoculation of transgenic PrP expressing HpL3-4 cell lines with CWD ...... 91

3.5 Inoculation of cynomolgus macaques with CWD tissue using different inoculation routes

\section{Discussion.}

4.1 CWD risk assessment using non-human primate in vivo and in vitro models.. 94

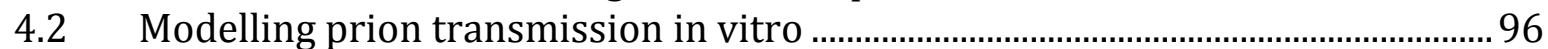

4.3 Overexpression of PrP in prion susceptible cell lines.............................................97

4.4 The amount of prion protein expression correlates with RNA expression data 99

4.5 Flow cytometric quantification of PrP presenting HpL3-4 and SH-SY5Y cell

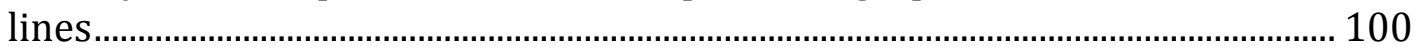

4.6 The tg(sai)PrP HpL3-4 cell line is susceptible to cell culture adapted 22L ..... 101

4.7 Susceptibility to prion infectious agents is cell line-dependent ......................... 102

4.8 Tg(sai)PrP HpL3-4 cells are susceptible to infection with primary CWD

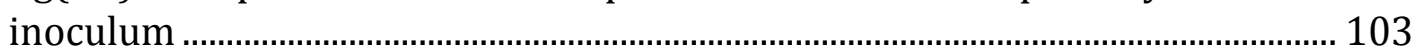

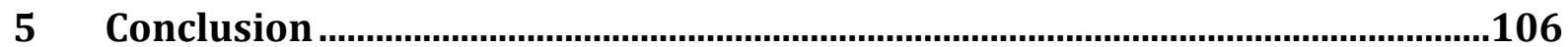

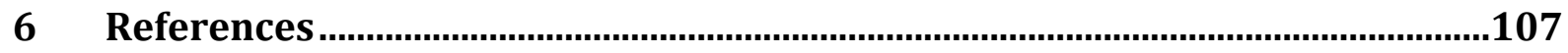

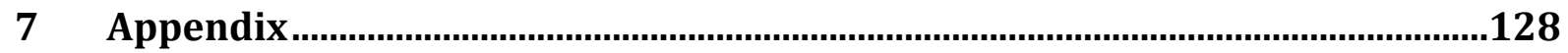

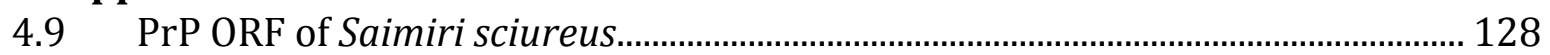

4.10 Prion protein sequence of Saimiri sciureus .......................................................... 128

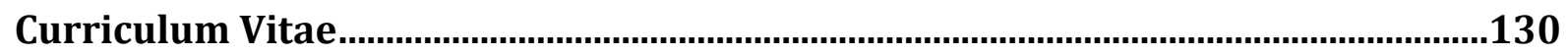

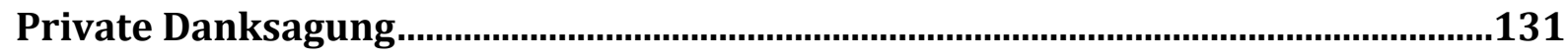




\section{List of Figures}

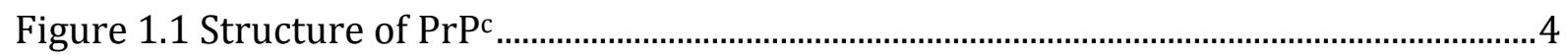

Figure 1.2 Diagnostic test for prion diseases..............................................................................

Figure 1.3 Chronic wasting disease (CWD) in North America ...............................................

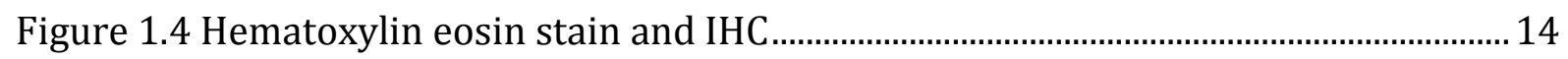

Figure 3.1 Amino acid alignment of squirrel monkey (S. sciureus) PrP ...............................56

Figure 3.2 EGFP expressing HEK293FT after transfection with pBABE-EGFP ...................59

Figure 3.3 Transduction efficiency of viral particles carrying EGFP transgene in HpL3-4

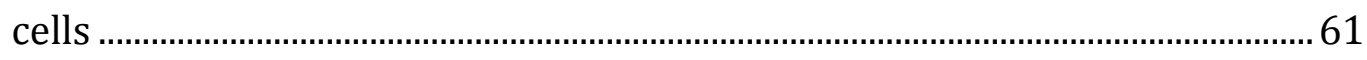

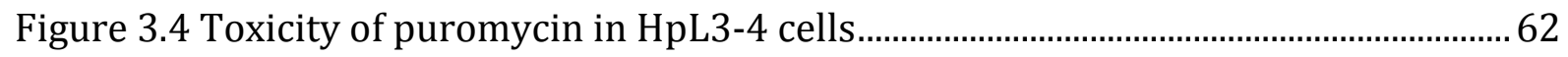

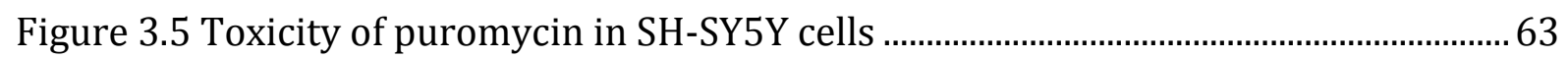

Figure 3.6 Expression of EGFP in HpL3-4 and SH-SY5Y cells after retroviral transduction

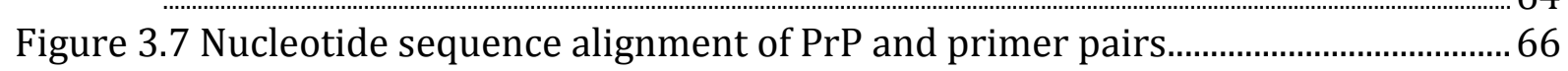

Figure 3.8 Number of transgenic PrP insertions in HpL3-4 and SH-SY5Y cells ................... 68

Figure 3.8 Ectopic expression of transgenic PrP in HpL3-4 and SH-SY5Y cells ................... 69

Figure 3.9 Alignment of ICSM-18 epitope ................................................................................... 70

Figure 3.10 Prion protein expression in transgenic HpL3-4 and SH-SY5Y cells................. 72

Figure 3.11 Conservation of monoclonal antibody epitopes among different species ..... 74

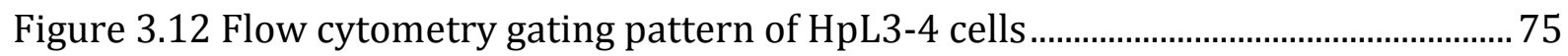

Figure 3.13 Specific detection of murine prion protein on $\operatorname{tg}(\mathrm{mo}) \operatorname{PrP}$ HpL3-4 cells by

flow cytometry 76

Figure 3.14 Influence of cell detachment procedures on the detection of surface-bound

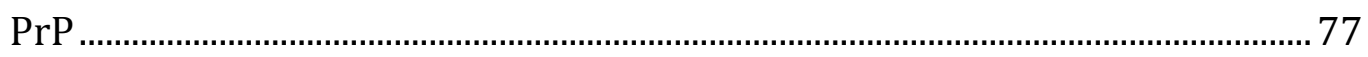

Figure 3.15 Surface abundance of prion protein in transgenic HpL3-4 cells........................ 79

Figure 3.16 Surface abundance of prion protein in transgenic SH-SY5Y cells ..................... 81

Figure 3.17 Detection of PrPres in 22L infected $\operatorname{tg}(\mathrm{mo}) \mathrm{PrP}$ HpL3-4 cells............................... 84

Figure 3.18 Influence of fetal calf serum on PrPres propagation in 22L infected

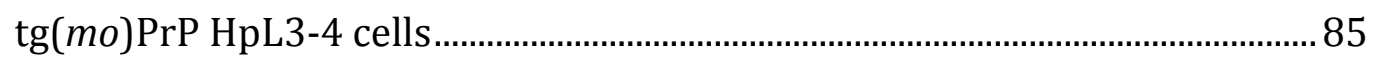

Figure 3.18 Infection of $\operatorname{tg}(\mathrm{mo}) \mathrm{PrP}$ HpL3-4 cells with cell culture adapted 22L................. 86

Figure 3.19 Infection of tg PrP HpL3-4 cells with cell culture adapted 22L.......................... 88

Figure 3.20 Inoculation of $\operatorname{tg}($ sai) PrP SH-SY5Y cells with cell culture adapted 22L .......... 90

Figure 3.21 Infection of $\operatorname{tg}($ sai $)$ PrP HpL3-4 cells with CWD brain ......................................... 92 


\section{List of Tables}

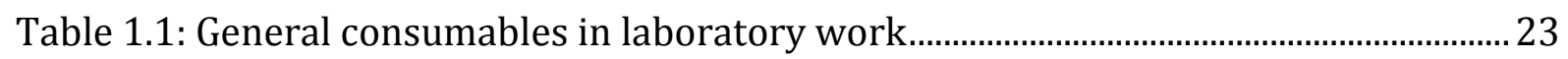

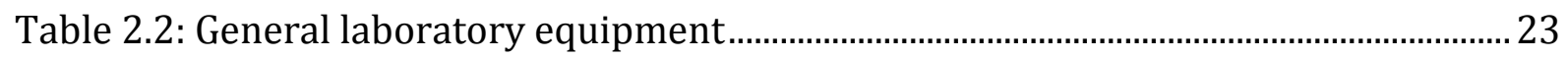

Table 2.3: General consumables and components for cell culture.......................................... 24

Table 2.4: General consumables for protein biology ............................................................. 25

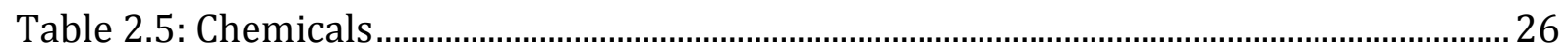

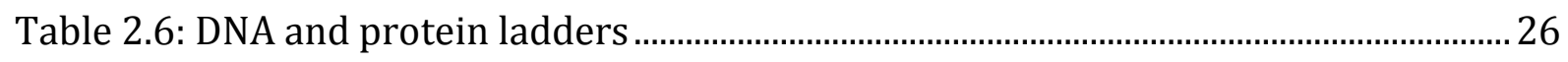

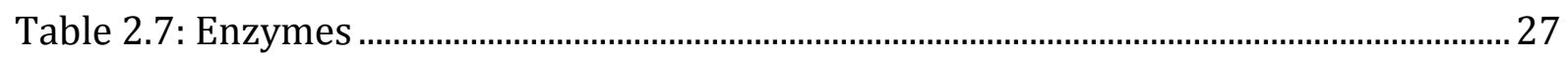

Table 2.8: Reaction components and commercial kits ............................................................ 27

Table 2.9: Antibodies with corresponding reference and application ................................... 29

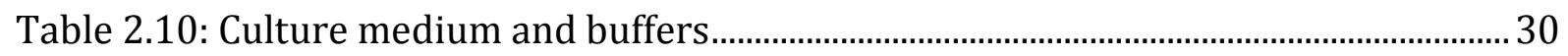

Table 2.11: Oligonucleotides for amplification............................................................................... 31

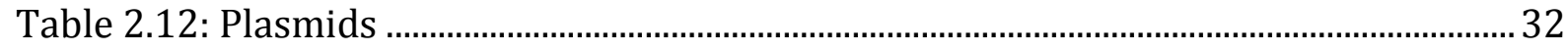

Table 2.13: Cell lines with corresponding reference and culture conditions ......................... 33

Table 2.14: Data analyses and software ........................................................................................ 51

Table 2.15: CWD inoculation study in M. fascicularis........................................................... 53

Table 3.1: Primer pairs for the amplification of PrP .................................................................. 55

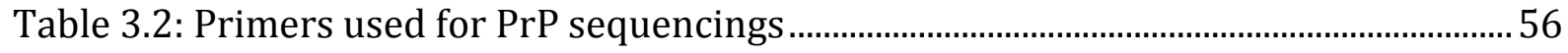

Table 3.3: Cloning primers for the amplification of PrP ……................................................... 57

Table 3.4: PrP transgenic cell lines generated by retroviral transduction ............................65

Table 3.5: Primer pairs for qPCR with corresponding standardisation plasmid ................66

Table 3.6: Mean fluorescence intensity for transgenic PrP presenting HpL3-4 cells ........ 80

Table 3.7: Mean fluorescence intensities for transgenic PrP presenting SH-SY5Y cells... 82 


\section{List of Abbreviations}

\begin{tabular}{|c|c|}
\hline Abbreviation & Explanation \\
\hline$\circ$ & Degree \\
\hline ‘ & Minute \\
\hline “ & Second \\
\hline AA & Amino acid \\
\hline Amp & Ampicillin \\
\hline APRI & Alberta Prion Research Institute \\
\hline $\mathrm{bp}$ & Base pair(s) \\
\hline BSE & Bovine spongiform encephalopathy \\
\hline BSL & Biosecurity level \\
\hline $\mathrm{C}$ & Celsius \\
\hline CD & Cluster of differentiation \\
\hline cDNA & Complementary DNA \\
\hline CJD & Creutzfeldt-Jakob-disease \\
\hline CMV & Cytomegalovirus \\
\hline $\mathrm{CO}_{2}$ & Carbon dioxide \\
\hline CSF & Cerebrospinal fluid \\
\hline CWD & Chronic wasting disease \\
\hline CWD ${ }^{\mathrm{ELK}}$ & Chronic wasting disease in elk \\
\hline CWDWTD & Chronic wasting disease in WTD \\
\hline $\mathrm{d}$ & $\operatorname{Day}(\mathrm{s})$ \\
\hline DEAE & Diethylaminoethyl cellulose \\
\hline DI $\mathrm{H}_{2} \mathrm{O}$ & Deionized water \\
\hline DMEM & Dulbecco's modified eagle medium \\
\hline DMSO & Dimethyl sulphoxide \\
\hline DNA & Deoxyribonucleic acid \\
\hline DNase I & DNA-hydrolyzing enzyme \\
\hline dNTP & Deoxynucleotide triphosphate \\
\hline DOC & Deoxycholic acid \\
\hline DPBS & Dulbecco's phosphate buffered saline \\
\hline dpi & Days post infection \\
\hline dpt & Days post transduction \\
\hline DPZ & Deutsches Primatenzentrum GmbH \\
\hline dsDNA & Double stranded DNA \\
\hline DSMZ & Deutsche Sammlung für Mikroorganismen und Zellkultur \\
\hline E.coli & Escherichia coli \\
\hline EDTA & Ethylenediamine tetraacetic acid \\
\hline EGFP & Enhanced green fluorescent protein \\
\hline EMC & Empty vector control \\
\hline
\end{tabular}




\begin{tabular}{|c|c|}
\hline env & Retroviral envelope protein \\
\hline FCS & Fetal calf serum \\
\hline FIU & Fluorescence intensity units \\
\hline FSC & Forward scatter \\
\hline Gag & Group-specific antigen, retroviral structure protein \\
\hline GAPDH & Glyceraldehyde-3-phosphate dehydrogenase \\
\hline gDNA & Genomic deoxyribonucleic acid \\
\hline GP & Gag, Pol (viral proteins) \\
\hline GPI & Glycosylphosphatidylinositol \\
\hline $\mathrm{h}$ & $\operatorname{Hour}(\mathrm{s})$ \\
\hline $\mathrm{H}_{2} \mathrm{O}$ & Water \\
\hline HEK & Human embryonic kidney (cell line) \\
\hline Homo & Human, Homo sapiens \\
\hline i.c. & Intracerebral \\
\hline IHC & Immunhistochemistry \\
\hline IRES & Internal ribosome entry site \\
\hline $\mathrm{kb}$ & Kilo base pairs \\
\hline $\mathrm{kDa}$ & Kilo Dalton \\
\hline LB & Lysogeny broth \\
\hline LDS & Lithium dodecyl sulfate \\
\hline LPS & Lipopolysaccharide \\
\hline LTR & Long terminal repeat \\
\hline $\mathrm{mAb}$ & Monoclonal antibody \\
\hline Mafa & Cynomolgus monkey, Macaca fascicularis \\
\hline MCS & Multiple cloning site \\
\hline $\min$ & Minute \\
\hline mRNA & Messenger RNA \\
\hline NCBI & National Centre for Biotechnology Information \\
\hline $\mathrm{NF}$ & Nuclease-free \\
\hline ORF & Open-reading frame \\
\hline PAGE & Polyacrylamide gel electrophoresis \\
\hline PBS & Phosphate-buffered saline \\
\hline PCR & Polymerase chain reaction \\
\hline $\mathrm{pH}$ & Potentia hydrogenii \\
\hline PK & Proteinase K \\
\hline pol & $\begin{array}{l}\text { Retroviral gene cassette encoding for reverse transcriptase, } \\
\text { integrase and protease }\end{array}$ \\
\hline PRNP & Gene coding fort he prion protein \\
\hline $\operatorname{PrP}$ & Prion protein \\
\hline $\mathrm{PrPc}^{\mathrm{c}}$ & Cellular prion protein \\
\hline PrPres & PK resistant prion protein \\
\hline PrPSc & Disease-associated prion protein \\
\hline puro & Puromycin \\
\hline
\end{tabular}




\begin{tabular}{ll} 
PVDF & Polyvinylidene fluoride \\
qPCR & Quantitative PCR \\
RKI & Robert-Koch Institute, Berlin \\
RNA & Ribonucleic acid \\
RNase & RNA-hydrolyzing enzyme \\
RT & Room temperature $\left(21^{\circ} \mathrm{C}\right)$ \\
RT & Reverse transcriptase \\
Sai & Squirrel monkey, Saimiri sciureus \\
SDS & Sodium dodecyl sulphate \\
SSC & Side scatter \\
SSDNA & Single stranded DNA \\
TBS & TRIS-buffered saline \\
TBS-T & TBS-Tween \\
tg & Transgene, transgenic \\
TRIS & Tris(hydroxymethyl)aminomethane \\
TSE & Transmissible spongiform encephalopathy \\
U & Unit \\
v/v & Volume/volume \\
VSV-G & Vesicular stomatitis virus G protein \\
w/v & Weight/volume \\
WB & Western blot \\
WT & Wild type \\
WTD & White-tailed deer, Odocoileus virginianus \\
Xg & Gravitation force \\
\hline
\end{tabular}




\section{Abstract}

The rapid spread and high prevalence of CWD in North American cervids have raised concerns about a potential risk to human health. Experimental transmission of CWD to various species has been reported. It was demonstrated by others, that CWD is transmissible to squirrel monkey but so far not to cynomolgus macaques. We investigated prion propagation in cell lines, overexpressing transgenic PrP from humans (Homo sapiens), cynomolgus macaques (Macaca fascicularis), squirrel monkeys (Saimiri Sciureus) and white-tailed deer (Odocoileus Virginianus) to simulate interspecies transmission of CWD in vitro. The permissive HpL3-4 and SH-SY5Y cell lines were retrovirally transduced with $\operatorname{PrP}$ of the respective species. We characterised the produced cell lines in detail for transgene integration, transcription, protein expression and cell surface abundance of ectopically expressed PrP. The relative and absolute number of integrated genomic copies and the transcription rate of transgenic PrP was comparable among all cell lines according to quantification by qPCR. Analysis of the glycopattern of transgenic prion protein expressing cell lines confirmed that glycosylation is not dependent on PrP donor species, but on the recipient host cell line. The abundance of transgenic PrP on the cell surface was validated by FACS analysis. All generated cell lines were inoculated with different prion infections agents and tested for prion propagation in vitro. The studies showed that only HpL3-4 cell lines, expressing the prion protein of mouse or squirrel monkey, were susceptible to infection with cell culture adapted Scrapie 22L cell lysate. In comparison, the tg PrP overexpressing SHSY5Y cell lines were not susceptible to $22 \mathrm{~L}$ infection. Additionally, the macaque and human HpL3-4 cell lines were not susceptible to infection with CWD However, the overexpression of PrP from the squirrel monkey in HpL3-4 cell line led to permissibility of the cells to primary CWD brain inoculum. This is the first report demonstrating that a transgenic PrP overexpressing cell line could be infected with a primary inoculate derived from CWD-infected cervids. The established in vitro assay partially reflects the in vivo transmissibility of CWD, which has been successfully transmitted to squirrel monkeys, but to date infection of cynomolgus macaques or humans by CWD has not been reported. In conjunction with a high number of replicates this assay provides a powerful tool to assist in vivo experiments in non-human primates, which could lead to a reduction and refinement of animal experiments. 


\section{Introduction}

\subsection{Prion diseases}

Prion diseases, also termed transmissible spongiform encephalopathies (TSEs), are fatal neurodegenerative diseases of the central nervous system (CNS) that affect a variety of animals as well as humans. The disease is accompanied by gliosis, neuronal loss and spongiosis and inevitably leads to the death of the host. Another characteristic of TSEs is the long incubation period, which can last for years or even decades (Collinge et al., 2006). TSEs are generally transmissible by infection. The pathogenesis is linked to the misfolding of the so-called prion protein, which is the causative agent of all known prion diseases.

TSEs were first observed in the $18^{\text {th }}$ century when sheep and goats developed a disease called Scrapie because of their rubbing and scratching against trees (Rüdiger, 1710). The most well-known TSE is bovine spongiform encephalopathy (BSE) in cattle, also known as "mad cow disease". First discovered in 1987 (Wells et al., 1987), the disease gained high public interest during the "BSE crisis" of the 1990's, when the United Kingdom reported hundreds of thousands of BSE cases. BSE was most probably transmitted to cattle via contaminated meat and bone meal (MBM). Consumption of cattle BSE products via diet can lead to variant Creutzfeldt-Jakob disease in humans. Spongiform encephalopathy not only affects cattle but is also found in mink (Marsh et al., 1969) and cats (Aldhous, 1990) as transmissible mink and feline spongiform encephalopathy. Chronic wasting disease (CWD) is classified as a prion disease and affects cervid species. Discovered in the 1970's CWD very recently became epidemic in North America (Williams and Young, 1980); the zoonotic transmission of CWD to humans is of particular concern.

Prion diseases also affect humans. These include Creutzfeldt-Jakob disease (Creutzfeldt, 1920; Jakob, 1921), Gerstmann-Sträussler-Scheinker syndrome (Gerstmann, 1936), fatal familial insomnia (Lugaresi et al., 1986) and Kuru (Gajdusek and Zigas, 1957). They can be sub-classified into familial, sporadic, acquired and iatrogenic forms of prion diseases, where the classification is related to the respective transmission route. Intraspecies transmission of Creutzfeldt-Jakob disease can occur. 


\subsection{The causative agent of prion diseases}

First described in 1982 by Stanley B. Prusiner, prions are small proteinaceous infectious particles with high stability against common inactivation procedures (Prusiner, 1982). The term "prion" has since been used as an acronym for proteinaceous infectious particle, which are assumed to be the causative agent of prion diseases. In contrast to common infectious agents like viruses, bacteria, fungi or parasites, Prusiner proposed that prions were infectious proteins due to the fact that the infectious agent was sensitive to alkali or lipase treatment and insensitive to antiviral treatment, e.g. methods that destroy or modify nucleic acids (Alper, Haig, and Clarke, 1978; Caughey and Raymond, 1991; Diener, McKinley, and Prusiner, 1982; Neary et al., 1991; Prusiner et al., 1981; Ward, Porter, and Stevens, 1974). It was further proposed, by Prusiner, that a misfolded prion could induce the misfolding of another prion (Prusiner et al., 1978). This induction of misfolding can lead to the accumulation of insoluble amyloids, which then aggregate to form fibrils and finally polymerise into large aggregates, facilitating the transmission and propagation of prion diseases.

The misfolded prion was discovered in 1982 as an insoluble protein that copurified with the Scrapie agent (Bolton, McKinley, and Prusiner, 1982). It exists in two different isoforms with distinct biochemical properties. The cellular prion protein $\left(\mathrm{PrP}^{\mathrm{c}}\right)$ is encoded by the host and expressed ubiquitously (Linden et al., 2008). The diseaseassociated, misfolded form of this protein is termed PrPSc (the Sc stands for "Scrapie", the first recognised prion disease). Both proteins, $\mathrm{PrP}^{\mathrm{c}}$ and $\mathrm{PrPs}^{\mathrm{s}}$, originate from the same primary amino acid sequence (Stahl et al., 1993).

The cellular prion protein, $\mathrm{PrPc}^{\mathrm{c}}$, is a highly conserved membrane-bound cell surface sialoglycoprotein (see figure 1.1) with a molecular weight of $\sim 35 \mathrm{kDa}$. All mammalian species, that have so far been analysed, show high similarity of the amino acid (AA) sequence and protein structure (Calzolai et al., 2005; Gossert et al., 2005; Lysek et al., 2005).

The human $\mathrm{PrP}^{\mathrm{c}}$ is encoded by the PRNP gene chromosome at $20 \mathrm{p} 13$ and consists of two exons (Basler et al., 1986; Oesch et al., 1985). The protein-coding region on exon 2 
results in a primary protein sequence of 253 AA in humans. Due to the posttranslational modifications, the AA sequence is shortened from 253 to 209 AA (human $\mathrm{PrP}^{\mathrm{c}}$ 23-231). The N-terminal signal peptide guides $\mathrm{PrP}^{c}$ to the endoplasmic reticulum. After cleavage $\mathrm{PrP}^{\mathrm{c}}$ is guided to the Golgi apparatus before it finally reaches the cell surface (Taraboulos et al., 1992). The prion protein is connected to the surface via a glycophosphatidyl inositol anchor (GPI-anchor), which is posttranslationally attached to the C-terminal AA serine 231 in human $\mathrm{PrP}^{c}$. Furthermore, the prion protein has two Nlinked glycosylation sites at asparagines 181 and 197 in human $\mathrm{PrPc}^{\mathrm{c}}$ (Rogers et al., 1990), which results in three different glycosylation forms: diglycosylated, monoglycosylated and unglycosylated. At the N-terminus of the protein, a repetitive region of eight AA (octarepeat) facilitates the binding of copper ions. In its secondary structure, monomeric $\mathrm{PrP}^{\mathrm{c}}$ forms a globular structure consisting of three $\alpha$-helices with two short antiparallel $\beta$-sheets. A disulfide bond is formed between helix two and three. The N-terminal polypeptide chain forms a random coil (Riek et al., 1997). PrPc can be endocytosed from the cell surface, cleaved and recycled to the surface (Caughey et al., 1991).

Although $\mathrm{PrP}^{c}$ is ubiquitously expressed, the highest expression of $\mathrm{PrP}^{\mathrm{c}}$ has been detected in the central nervous system (Oesch et al., 1985), with varying amounts found in distinct brain regions (Brown et al., 1998a; Brown, Schmidt, and Kretzschmar, 1996; Giese et al., 1998). In brain regions $\mathrm{PrP}^{c}$ is predominantly located at synaptic membranes (Herms et al., 1999). In addition, $\mathrm{PrP}^{c}$ is expressed in a variety of nonneuronal cells (Brown et al., 1998b; Brown et al., 1990).

Due to the lack of a clear and instructive phenotype of $\mathrm{PrP} / 0$ mice (Tremblay et al., 1998) the ultimate function of $\mathrm{PrP}^{\mathrm{c}}$ is still debated. It is known that $\mathrm{PrPc}^{\mathrm{c}}$ can bind to copper ions in physiological concentrations (Brown et al., 1997; Kramer et al., 2001). This presumably provides an anti-oxidative effect against superoxids and reactive oxygen species in neurons (Brown, Schmidt, and Kretzschmar, 1997). The prion protein is also involved in the metabolism of other metal ions such as zinc (Watt and Hooper, 2003) or manganese (Kralovicova et al., 2009). A detailed description of recent findings on the proposed physiological function of $\mathrm{PrP}^{c}$ and an entire expression overview is given in the review by Rafael Linden and colleagues (Linden et al., 2008). 


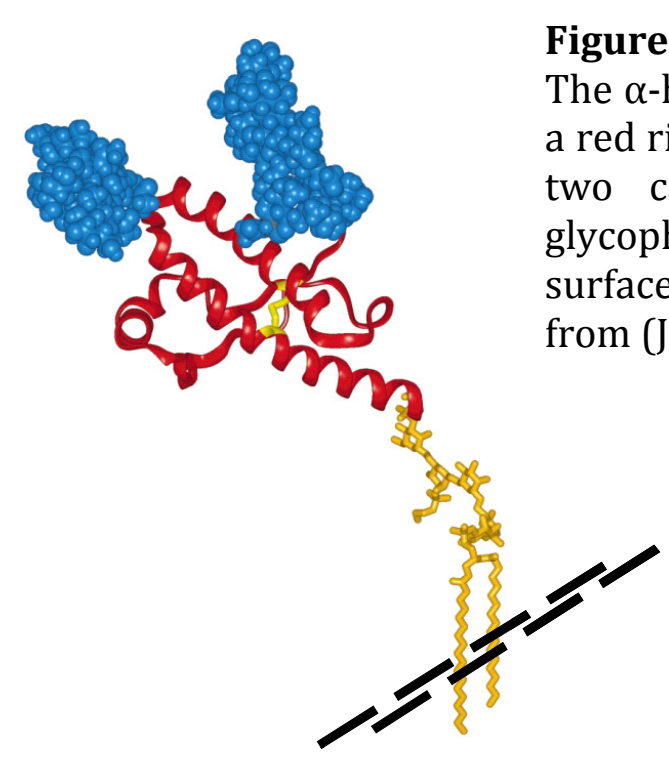

The pathological isoform of the prion protein is termed $\operatorname{PrPscrapie}\left(\mathrm{PrPs}^{\mathrm{Sc}}\right)$, diseaseassociated PrP $\left(\mathrm{PrP}^{\mathrm{d}}\right)$ or proteinase $\mathrm{K}(\mathrm{PK})$ resistant PrP (PrPres). According to the classification of Victoria Lawson and colleagues (Lawson et al., 2005) PrPSc describes a disease-associated isoform of PrP, which is directly linked to infectivity. In comparison, PrPres describes a PK resistant isoform of $\mathrm{PrP}$, which is generated in vitro, implying that it is not necessarily associated with infectivity. It is generally believed that the pathological isoform $\mathrm{PrPSc}^{\mathrm{Sc}}$ is generated by a conformational change that converts $\mathrm{PrP}^{\mathrm{c}}$ to PrPSc.

In contrast to the $\alpha$-helical-rich structure of $\mathrm{PrP}^{\mathrm{c}}$, the tertiary structure of PrPsc shows a higher content of $\beta$-sheets (Riesner, 2003). The misfolded PrPSc molecule seems to trigger formation of oligomeric structures, which then tend to form fibrils (Silveira et al., 2005; Walsh and Selkoe, 2004). The conversion to PrPSc therefore induces the aggregation of PrPSc in the CNS. Several reports provide evidence that nucleic acids and nucleic acid binding proteins could be involved (Caughey and Kocisko, 2003; Deleault, Lucassen, and Supattapone, 2003; Lima et al., 2006; Marques et al., 2009; Sklaviadis et al., 1993). The triggering mechanism or identity of supportive co-factors of this misfolding remains largely unknown (Fasano, Campana, and Zurzolo, 2006; Kupfer, Hinrichs, and Groschup, 2009). Due to the conversion to a misfolded $\beta$-sheet-rich structure and subsequent aggregate formation, the $\operatorname{PrPsc}$ molecule gains partial resistance to PK (Jendroska et al., 1991; Johnson et al., 2011). This partial resistance is used to discriminate between $\mathrm{PrPc}^{c}$ and $\mathrm{PrPSc}$ (see figure 1.2). 


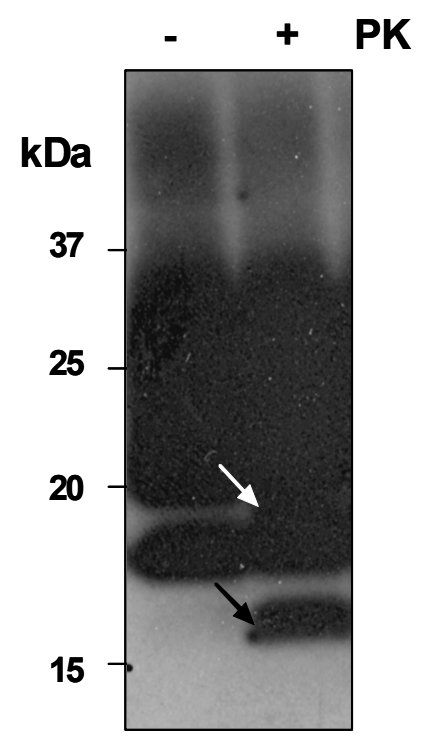

Figure 1.2: Diagnostic test for prion diseases

A scrapie infected cell lysate was processed and treated with proteinase $\mathrm{K}(\mathrm{PK})$. After electrophoretic separation (PAGE) and immunoblotting with an anti-prion antibody, PK resistant $\mathrm{PrPSc}^{(+)}$can still be detected due to the partial resistance of the misfolded protein. The shift in the molecular mass indicates the shortening of the PK resistant prion protein. 


\subsection{Prion diseases of animals}

As discussed above, prion diseases can affect animals, like sheep and goats (Scrapie), cattle (BSE) and cervids (CWD). Furthermore, TSEs can also occur in feline species (FSE), mink (TME) and zoo animals (Kirkwood and Cunningham, 1994).

The first written English scientific reference for a prion disease was already made in the 1920s for Scrapie in sheep and goats (McGowan, 1922). Actually the first description was already given in 1710's in German (Rüdiger, 1710). Scrapie only affects sheep and goats and since its discovery in the 1710's has spread all over the world, mainly in countries with large sheep populations. The endemic and infectious etiology, which leads to the classification as a transmissible disease, was demonstrated by the transmission of Scrapie from one sheep to another (Cuille, 1939). It has also been demonstrated that Scrapie can cross the species barrier with transmission to rodents and squirrel monkeys (Gibbs et al., 1980; Zlotnik and Rennie, 1962; Zlotnik and Rennie, 1965).

The Scrapie agent is widely used in TSE research. Scrapie strains, such as 22L (Kim et al., 1990a), RML (Carlson et al., 1994) and others, were generated by the transmission of Scrapie inoculum from infectious sheep to susceptible mice or hamster. The Scrapie strains provide a model system for the estimation of conversion from $\mathrm{PrPc}^{\mathrm{c}}$ to $\mathrm{PrPsc}$ (Kocisko et al., 1995), generation of amyloid fibrils (Prusiner et al., 1983), co-factor analysis (Fasano, Campana, and Zurzolo, 2006), transmission studies across the species barrier (Race et al., 1995; Scott et al., 2005) and also treatment strategies (Dealler and Rainov, 2003; May et al., 2003; Pocchiari, Schmittinger, and Masullo, 1987).

The clinical signs of Scrapie in sheep and goats are diverse and include loss of coordination and behavioural changes. The most characteristic symptom is the pruritus (scraping or scratching) that led to the naming of the disease. The histopathology of the brain shows neuronal degeneration and loss and PrPres deposition primarily in the medulla oblongata (Wood et al., 1997). For sheep Scrapie the PrP genotype influences the susceptibility (Dawson, Moore, and Bishop, 2008). Therefore huge genotyping approaches with selection for Scrapie 'resistant' genotypes had been carried out. This 
enabled spreading of atypical forms. Besides the classical Scrapie, another variant of Scrapie was detected in 1998 and termed as atypical/Nor98 Scrapie (Benestad et al., 2003). Compared to classical Scrapie, atypical Scrapie shows distinct genetic, biochemical and histopathological features. Due to these properties, the diagnosis of atypical Scrapie has been challenging.

The bovine spongiform encephalopathy (BSE), also called 'mad cow disease', is a fatal neurodegenerative disease, which affects cattle. The disease was first discovered in 1987 (DEFRA, 2000; Wells et al., 1987). During the 1990's there were a number of large scale BSE outbreaks, when a huge number of animals got infected by the consumption of meat-and bone meal (MBM) from infected cattle (Nathanson, Wilesmith, and Griot, 1997). The most affected country during the BSE epidemic was the United Kingdom with nearly 200,000 affected animals diagnosed in 1992 alone (VLA, 2011). To date BSE cases have occurred world-wide in 24 different countries. Due to extensive eradication programs all over Europe, more than 4.4 million cattle were culled. Bovine offal and MBM has also been banned from farm animal feeds since 1996. Fifteen years after the feed ban there are still BSE cases being diagnosed, suggesting that there are additional routes for BSE transmission such as environmental reservoirs (Gough and Maddison, 2010). The first symptoms of BSE are abnormalities in gait, tremors, loss of body weight and behavioural abnormalities (Braun et al., 1998; Cockcroft, 2004; Wilesmith et al., 1988). Histopathological evaluation reveals vacuolar lesions in the brain stem, gliosis and neuronal loss (Simmons et al., 1996).

Interspecies transmission of cattle BSE was shown in several other animal models (Bruce et al., 1994; Collinge et al., 1995; Foster et al., 1996). A vertical maternal transmission of BSE to new-borns has not yet been detected, but transmission via milk replacers, which contain animal fats, was observed (Clauss et al., 2006). The exposure of to BSE-contaminated products is causally linked to variant Creutzfeldt-Jakob disease in humans.

In comparison to human and sheep, genetic predisposition for BSE was not observed in cattle, although two genotypes of the bovine PRNP with varying length in the octapeptide region have been reported (Hunter et al., 1994). An influence in progression 
and age of onset cannot be excluded (Castilla et al., 2004; Croes et al., 2004). The diagnosis of BSE in cattle is still not possible in live animals due the lack of a blood test with sufficient sensitivity. As with Scrapie, atypical forms of BSE, that produce abnormal symptoms, have been demonstrated (Buschmann et al., 2006). So far, two atypical BSE forms have been described (Biacabe et al., 2004; Casalone et al., 2004) These forms of BSE are transmissible to primates (Comoy et al., 2008). The origin of BSE is unclear, but it has been suggested that BSE arose due to interspecies transmission of Scrapie to cattle (Bruce et al., 1994).

Chronic wasting disease (CWD) was first discovered in 1967 in captive mule deer at a research facility in Colorado (Williams and Young, 1980). CWD is the only TSE that has so far been detected in wild animals. In 1985 it was classified as a TSE in cervids, including mule deer (O. hemionus), white-tailed deer (O. virginianus), Rocky Mountain elk (C. elaphus nelsoni) and less frequently moose (A. alces). Currently, CWD has been diagnosed in 14 American and Canadian provinces in captive and wild cervid populations (see figure 1.3). Over the last decade, the geographical spread of CWD has increased tremendously. Endemic areas with a prevalence of up to $90 \%$ in captive and $50 \%$ in free-ranging cervids have been reported (Williams, 2005). Programs to lower or eradicate CWD have not, so far, been successful, most likely due to the long asymptomatic preclinical phase, the unpredictable migration of the shy animals and the environmental stability of CWD-infectivity. The clinical signs of CWD include progressive weight loss, salivation, ataxia with head tremor (Spraker et al., 1997) at the mean age of 2-8 years. The slowly progressing symptomatic phase lasts 5-12 months (Miller, Wild, and Williams, 1998) with males and females being equally affected.

The histopathology of CWD is generally consistent with other animal prion diseases. The histopathological changes can be found in the CNS with spongiform lesions, neuronal loss, astrocytic hypertrophy and amyloid plaques (Williams and Young, 1993). In addition, abnormalities in $\mathrm{PrPSc}$ deposition along the small and large intestine and spleen have been demonstrated (Sigurdson et al., 1999). In experimentally infected animals, accumulation of $\mathrm{PrPs}$ in tonsils and lymph nodes was already detectable after 90 days post infection. Furthermore, deposition of PrPSc was measurable in the muscle tissue of infected animals (Angers et al., 2006; Jewell et al., 2006) as well as blood, saliva, 
urine, faeces and antler velvet (Angers et al., 2009; Mathiason et al., 2006; Safar et al., 2008), implicating an environmental contamination with cervid prions. Genetic polymorphism of cervid PRNP (Q95H, G96S, A116G, M132L, S138N) has not been correlated with complete resistance to CWD-infection (Johnson et al., 2003; O'Rourke et al., 1999). Recently, two different strains of CWD were discovered in cervidized mice causing distinct neuropathological profiles with similar biochemical signatures (Angers et al., 2010). A remarkably high proportion of horizontal transmission seems to occur by direct contact between the animals or via contact with contaminated saliva or faeces (Miller and Williams, 2003). Interspecies transmission to transgenic mice (Raymond et al., 2007), ferrets (Perrott et al., 2011) and squirrel monkeys (Race et al., 2009) was reported. The origin of CWD remains unclear. It has been suggested that CWD occurred spontaneously (Miller et al., 2004) or arose due to the consumption of contaminated material in feeding stations or was the result of interspecies transmission from Scrapieinfected sheep (Hamir et al., 2004b).

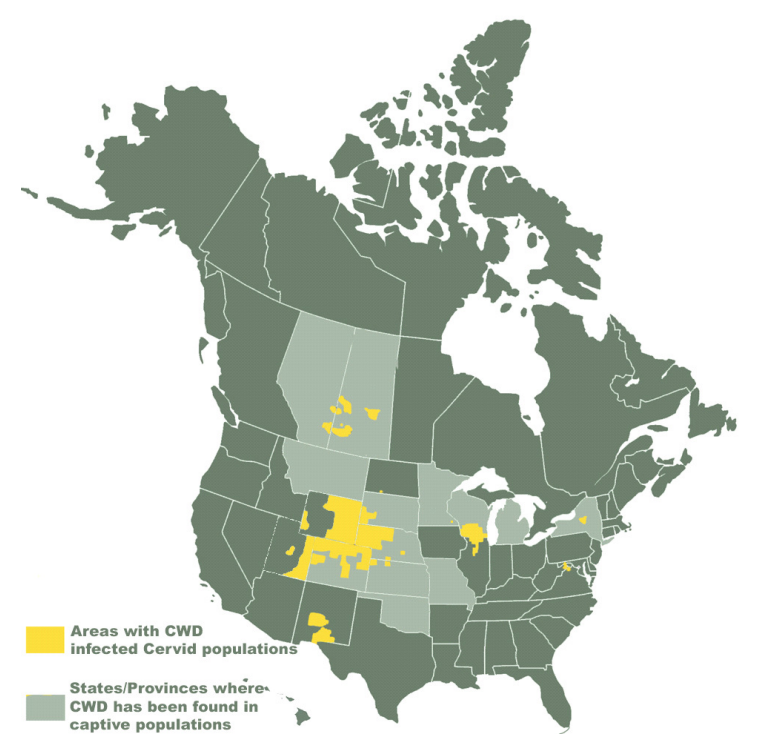

\section{Figure 1.3: Chronic wasting disease (CWD) in North America}

The map shows the distribution of CWD on the North American continent for February 2011. Yellow indicates areas with CWD-infected free-ranging cervid populations. Light grey indicates 17 states (USA) and 2 provinces (Canada) where CWD has been diagnosed in the captive cervid populations. The map was adapted from www.cwd-info.org. 


\subsection{Prion diseases in humans}

Human prion diseases are fatal neurodegenerative disorders. The most abundant form is sporadic Creutzfeldt-Jakob-disease (sCJD). The physicians Hans Gerhard Creutzfeldt and Alfons Maria Jakob first discovered CJD and described the disease pattern as a "particular disease of the central nervous system" (Creutzfeldt, 1920; Jakob, 1921). The disease comes in four different forms: sporadic (sCJD), familial (fCJD), iatrogenic (iCJD) and variant (VCJD). The most common form of the disease in the human population is sCJD, with a incidence of 1-2:1,000,000 (Masters et al., 1979), for people aged 60-70 years and an equal sex distribution. The disease pattern is characterised by a long asymptomatic incubation period of 10-30 years, followed by a preclinical phase with continuous loss of cognitive and motoric abilities over a period of 4-6 months, which inevitably leads to death.

The most relevant polymorphism in the PRNP gene encoding for the prion protein was detected at codon 129 (Owen et al., 1990) encoding for Methionine or Valine with a distribution of $38 \% \mathrm{MM}, 11 \% \mathrm{VV}$ and $51 \% \mathrm{MV}$ in the northern European population (Budka, 2007). More than 30 pathogenic mutations are known for the human PRNP gene including octapeptide repeat insertions, deletions and various point mutations causing missense or stop substitutions (Kovacs et al., 2002; Mead, 2006). Most of the mutations are associated with hereditary prion diseases and can be monitored by sequencing of the PRNP gene.

Using molecular criteria such as the different size of the unglycosylated prion protein fragment after Proteinase K (PK) digestion (Collinge, 1997; Collinge, 2005; Parchi et al., 1996; Parchi et al., 1999), CJD can be divided into two different types (CJD type I/II). Type I and type II have different clinicopathological patterns and distinct neuropathological lesion profiles. The combination of both CJD types (type I and type II) together with the different genotypes at codon 129 (MM, VV, or MV) results in six clinical relevant forms of CJD (Owen et al., 1990; Parchi et al., 1999). The terminology 'strain' and 'type' will frequently be used in this thesis. So far, there is no standard definition for either term (Bruce, 2003; Vorberg and Priola, 2002; Wadsworth et al., 2008). For the purpose of this thesis the term 'strain' will solely be used when the 
incubation time and the lesion profile of a prion-infectious agent have been defined in a different species. In contrast, the term prion "type" will be used when a prion-infectious agent shows distinct biochemical properties in one species, e.g. glycopattern, length variation, stability or deposition pattern in the brain (Wemheuer et al., 2009).

The clinically relevant parameters for CJD are the same as for all TSEs, including the histopathological characteristics of spongiosis, gliosis and neuronal decay. The resulting symptoms include progressive dementia, cerebellar ataxia, pyramidal signs, chorea, myoclonus and others that appear in variable combinations. A detailed description of the phenotypes with corresponding PrPSc deposition and distribution has been given elsewhere (Budka, 2007; Watzlawik et al., 2006). The diagnostic discrimination between sporadic and acquired forms of CJD relies on the neuropathological examination of the PrPSc deposition in the CNS.

Gerstmann-Sträussler-Scheinker syndrome (GSS) can be transmitted by autosomal dominant inheritance. The name originates from its three discoverers Josef Gerstmann, Ernst Sträussler and Ilya Scheinker (Gerstmann, 1936). It is caused by a mutation in the PRNP gene at codon 102 that leads to the exchange of proline to leucine. In addition, GSS can also be caused by other point mutations within the gene (P105L, A117V, G131V, F198S, D202N), octapeptide insertions or nonsense mutations (Jansen et al., 2011; Piccardo et al., 1998; Piccardo et al., 2001; Vital et al., 2011). GSS is typified by the early onset of cerebella ataxia with a late development of dementia (Mastrianni, 1998). A hallmark of this hereditary encephalopathy is the formation of multi-centric PrPSc plaques (Liberski and Budka, 2004) and the shorter (7-14 kDa) unglycosylated fragment of PrP. Besides full-length PrP, a truncated form of PrP (PrP $58-150)$ can be detected in amyloid plaques (Chen et al., 1995; Parchi et al., 1998a; Piccardo et al., 1995). The clinical pattern is highly polymorphous, even among families carrying the same genotype and makes the diagnosis and classification difficult. The prevalence is estimated to $1-10$ cases/100,000,000.

Fatal familial insomnia (FFI) is an autosomal dominant inherited human prion disease, first described in 1986 in an Italian family and is described as a thalamic dementia. It is predominantly caused by mutations in the PRNP gene at codon 178 (D178N) with 
linkage to methionine homozygosity at codon 129 (Medori et al., 1992). The onset of disease occurs during middle age, starting with sleeplessness, dysautonomia and disturbance in the circadian rhythm (Benarroch and Stotz-Potter, 1998; Lugaresi et al., 1986; Montagna et al., 2003) rapidly leading to fatal insomnia. In comparison to other forms of CJD widespread spongiform degeneration does not occur and PrPres is hardly detectable. Immunoblotting shows an underrepresented unglycosylated PrP fragment (Parchi et al., 1998b). According to experimental and clinical data a prion protein dysfunction has been proposed (Tateishi et al., 1995). Besides the inherited form of FFI, the disease can also occur sporadically (Gambetti and Parchi, 1999).

Prion diseases such as variant CJD (vCJD), iatrogenic CJD (iCJD) and Kuru are acquired diseases. They are transmitted by the consumption of prion-infectious agents, in the case of vCJD and Kuru or by intra- or interspecies iatrogenic transplantation in iatrogenic CJD (iCJD). In comparison to the sporadic forms of CJD, iCJD occurs via transmission of contaminated biological products during medical procedures, such as treatment with growth hormone, transplantation of dura mater graft or cornea, or general contamination of surgical instruments with PrPsc (Brown, 2000). Medical products derived from cattle such as collagen and somatropin are also potential risk factors for iCJD.

Since 1996 an additional form, variant CJD (vCJD) has been observed, with 224 vCJD cases recorded worldwide (EUROCJD, 2011). This form is most probably caused by the consumption of BSE-contaminated cattle products. The histopathological features of vCJD are highly consistent and differ from other human prion diseases (Ironside and Head, 2004; Will et al., 1996). In addition, the median age for disease onset is at 28 years much lower than that of CJD (Belay and Schonberger, 2002). The transmission by blood transfusion was observed in four preclinical vCJD blood donors (Llewelyn et al., 2004; Peden et al., 2004), but is hotly debated. The post mortem investigations were not consistent, showing clinical vCJD related symptoms in 2 cases, abnormal prion protein in the spleen and lymph node, but not in the brain and no pathological features of vCJD in another case. All four patients received transfusions of non-leucodepleted red blood cells. For this reason leucodepletion is mandatory for all blood transfusions in the UK since October 1999. The need for a sensitive blood test to detect preclinical vCJD 
carriers is under debate (Edgeworth et al., 2011). The genotype at codon 129 affects susceptibility to vCJD and has also a huge impact on disease progression in SCJD (SchulzSchaeffer et al., 1996). Only the genotype MM has been found in patients affected by vCJD.

Kuru is another acquired TSE that was first discovered in the 1950's among cannibalistic groups in the Fore tribe of the eastern highlands of Papua New Guinea. A group of physicians including Daniel C. Gajdusek and Vincent Zigas did the first detailed research on Kuru (Gajdusek and Zigas, 1957). The clinical hallmarks of this disease are shivering (original Fore translation of "Kuru") and severe cerebella ataxia accompanied with tremors (Hornabrook, 1968; Hornabrook and Field, 1969). Gajdusek postulated that the spread of the disease within the tribe may have been caused by their cannibalistic funeral practices. The female and young Fore people consumed the viscera (including CNS tissue) of deceased relatives, leading to an annual prevalence of up to $10 \%$ in some Fore villages, mainly in woman (the men ate less infectious tissues like muscle). The transmissibility of Kuru to mice and to primates has also been demonstrated by Gajdusek and colleagues (Field, 1968; Gajdusek, Gibbs, and Alpers, 1966). In 1976 Gajdusek and Baruch S. Blumberg received the Nobel Prize in Physiology for "discoveries concerning new mechanisms for the origin and dissemination of infectious diseases". Since the funeral practice of the eating of the deceased was banned, the incidence of Kuru has continuously decreased. However, 40 years later single cases of Kuru could still be diagnosed (Collinge et al., 2008). 


\subsection{Diagnosis of Prion diseases}

To date, final diagnosis of TSE in animals or humans is only possible post mortem on examination of the brain. Depending on the classification, each TSE exhibits characteristic features, which confirm or exclude certain types and strains of prion diseases. However, TSEs in some animals can be diagnosed ante mortem after the appearance of clinical symptoms. In these cases, biopsies taken from the lymphoreticular system, e.g. recto-anal mucosa associated lymphoid tissue (RAMALT) for CWD (Keane et al., 2009) or lymph node biopsies taken in classical Scrapie or vCJD cases (Diener, McKinley, and Prusiner, 1982; Gonzalez et al., 2008; Kubler, Oesch, and Raeber, 2003; O'Rourke et al., 2000; Schreuder et al., 1998; Soto, 2004; Wild et al., 2002; Wolfe et al., 2007), are used to detect PrPSc. Nevertheless, rapid and versatile non invasive intra vitam tests have not yet been developed. The following section provides an overview of the most common techniques for the validation of prion diseases.

Histopathological examination of the brain or CNS sections (see figure 1.4) can be used to identify characteristic spongiform lesions, gliosis, and the presence of plaques (Wells, 1993). The processing and fixation in diagnostics requires 3-14 days and is not appropriate for rapid and large-scale diagnostics.
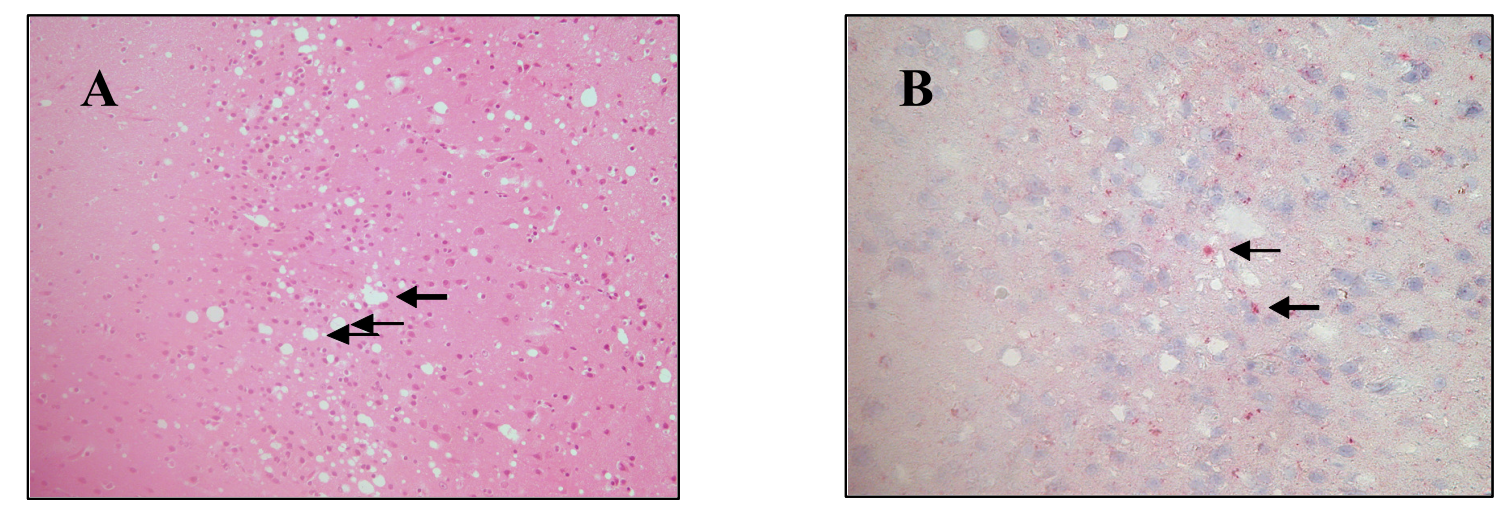

Figure 1.4 A: Hematoxylin and eosin staining of gyrus cinguli in BSE-infected macaque brain. Arrows mark Spongiform lesions. B: IHC staining of perivacuolar and intraneuronal disease associated Prion deposits ( $\mathrm{PrPSc}$ ) are located next to the spongiform lesions (arrows). The pictures were kindly provided by Drs. Motzkus and Schulz-Schaeffer. 
Immunohistochemistry (IHC) is based on the microscopic examination of CNS sections, either cryo-fixed or embedded in paraffin, which are cut into thin sections. PrPres accumulation is subsequently detected in the tissue sections by immunoreactivity to a prion specific antibody. Non-infectious $\mathrm{PrP}^{\mathrm{c}}$ is discriminated from PrPres by additional proteinase K digestion (Brown, Schmidt, and Kretzschmar, 1996). The sensitivity and specificity of this technique strongly depends on the preparation, fixation and choice of prion antibody (Hardt, Baron, and Groschup, 2000).

Enzyme-linked immunosorbant assay (ELISA) tests have been developed and commercialised. The ELISA is an immunological method to demonstrate the presence or absence of a certain antigen by the binding of an antigen-specific immunoreactive antibody and its detection via a chromogenic substrate (Engvall and Perlmann, 1971). This method is an accredited technique and is widely used in prion research for rapid testing of animal TSEs. The advantages are a high sample throughput and a fast result. The main disadvantageous is the missing detailed information about the nature of PrPres, e.g. strain or type.

Biochemically, PrPres can be detected by Western blot analysis. This technique was developed to demonstrate and visualise the abundance of a certain protein in a complex sample (Burnette, 1981; Laemmli, 1970) by the use of specific antibodies. The prion protein has three different glycosylation forms (di-, mono-, and unglycosylated). The glycoprotein can be visualised after electrophoretic separation, blotting to a membrane and subsequent immunoreactivity to a prion specific antibody. To discriminate $\mathrm{PrP}^{\mathrm{c}}$ from PrPres the sample is digested with PK. The core of the disease-associated PrPres is partially resistant to the treatment with PK. The N-terminally shortened form of PrPres and its respective glycoforms $\operatorname{PrP}(27-30 \mathrm{kDa})$ can be detected whereas $\operatorname{PrP}^{\mathrm{c}}$ is completely degraded (Madec et al., 1997). The ratio of the three glycoforms is used to distinguish between certain prion types of TSE (Parchi et al., 1996; Vorberg and Priola, 2002). The unglycosylated fragment has varying lengths after PK digestion, which is also a characteristic feature of different prion strains and TSEs (Parchi et al., 1999). Furthermore, different antibodies specifically bind to certain epitopes of the prion protein and can be used to discriminate between prion proteins derived from different species. 
The above-mentioned diagnostic tests are used to detect a PK resistant PrP isoform (PrPres). PrPSc associated infectivity can only be confirmed by in vivo bioassays. Therefore, the analysed tissue of interest is homogenised and injected into the brain of a recipient animal in decreasing dilutions. After observation of clinical signs detection of $\mathrm{PrP}^{\mathrm{Sc}}$ is accomplished by methods such as IHC or Western blot and the infectivity of the analysed tissue can be determined. The incubation time is in general proportional to the inoculated infectious dose. The bioassay can also be used to distinguish prion strains (Aguzzi, Heikenwalder, and Polymenidou, 2007; Bruce, 2003) (Thackray et al., 2007), or to investigate the potency of anti-prion drugs (Di Bari et al., 2008). Usually the recipient animals are rodent species, but evolutionary higher animals, like cattle or cervid species, have been used for titration of infectious tissue as well as the sensitivity of the bioassay has been increased by the use of transgenic mouse models that overexpress the respective $\mathrm{PrPc}^{\mathrm{c}}$ of a certain species (Buschmann et al., 2000; Le Dur et al., 2005). The advantage of bioassays is the sensitive detection of infectivity, the sensitivity also depends on the mouse strain used (Safar et al., 2002). 


\subsection{Animal models in Prion research}

To elucidate the physiological function of PrP the most instructive model to date far was the generation of a PrPo/0 mice (Bueler et al., 1992; Weissmann et al., 1994b). These mice exhibited a mild phenotype with learning deficits, irregularities in circadian rhythm and others (Brown et al., 1997; Tobler et al., 1996; Tremblay et al., 1998). The mice have demonstrated resistance to prion infection, which identified the TSE associated gene (Bueler et al., 1993; Weissmann et al., 1994a).

Prions, or the pathological prion protein, are postulated to be transmissible by proteinmediated transfer. Although intensive research has been accomplished using rodent models, the mechanism of propagation or factors, which may contribute to the interspecies crossing of a TSE have not been identified so far. Transgenic mouse models have also been used to analyse the species barrier of TSEs.

Knock-in of PrPc from the analysed species of interest into PrP ablated mice are used for TSE interspecies transmission experiments. These animals express transgenic PrP and are further inoculated with prion-infectious agent to test interspecies transmission (Foster et al., 1994; Windl et al., 2005). These models, however, have their limitations when used to simulate interspecies transmission to humans (Scott et al., 1993). So far, it was not possible to transmit BSE to bovinized mice (Bishop et al., 2006), contrary to the in vivo situation. Therefore, other animal models were used to ascertain or exclude the transmissibility to another species.

Non-human primate models are useful to evaluate and investigate the risk of nonhuman TSEs to human health. This was demonstrated with the BSE susceptibility of cynomolgus macaques (Lasmezas et al., 1996). Intensive research has been carried out to model prion infectivity in primates. The use of primates to test the transmissibility of TSEs to humans was pioneered by Gajdusek and colleagues. They were the first to estimate the potential risk of Scrapie, Kuru and CJD (Gibbs et al., 1980) in humans by inoculating squirrel monkeys with these infectious agents. Subsequent studies were accomplished in a wide range of primate and non-human primate species, including cynomolgus macaques, rhesus macaques, African green monkeys, squirrel monkeys and 
chimpanzees (Gibbs et al., 1980; Gibbs and Gajdusek, 1972; Gibbs et al., 1968). Although chimpanzees show the closest genetic relationship to humans, TSE transmission was banned for ethical reasons (Novakofski et al., 2005). Since then, intense research has been accomplished in non-human primates to estimate the transmissibility to humans. To date, all known TSEs have been successfully transmitted to non-human primates. The results show that the transmissibility is strictly dependent on the origin of the infectious agent and on the recipient species. Successful transmission of Scrapie, BSE, CJD and CWD has been demonstrated in squirrel monkeys (Gibbs et al., 1980; Marsh et al., 2005) indicating a low species barrier for this model organism.

For evaluation of the zoonotic potential of TSE to humans, the cynomolgus macaque has been used (Comoy et al., 2008; Gibbs and Gajdusek, 1972; Herzog et al., 2005; Herzog et al., 2004; Lasmezas et al., 2005). Transmission of atypical BSE to cynomolgus macaques was also demonstrated, indicating the importance of non-human primate models (Comoy et al., 2008). Such cases are often not detected in surveillance programs, due to their non-characteristic features and may easily get into the human food chain. Limiting factors for the use of non-human primate models are the small group number, the noninbred genetic background, the long preclinical incubation time (up to 10 years) and the highly elaborate and cost intensive animal husbandry under BSL-2 or BSL-3 conditions. 


\subsection{Cell based models in prion research}

Cell cultures are versatile and useful experimental models that provide a very controlled system to investigate cellular processes. Prion research cell culture models are considered to be powerful tools in studying the biology of PrPres including conversion, aggregation and degradation. Furthermore, interspecies transmissibility may be simulated in cell based assays as an alternative to in vivo models.

The first reports describing cell based assays were published in the 1970's (Clarke and Haig, 1970), when primary cells derived from Scrapie infected mice were cultured and PrPres was detected at a low level. In the early 2000's a few cell lines were described, which were permissive for rodent-adapted Scrapie strains (Solassol, Crozet, and Lehmann, 2003). The N2a (Butler et al., 1988), CAD (Mahal et al., 2007) and GT1 (Schatzl et al., 1997) cell lines were shown to be permissive for a wide range of Scrapie agents derived from laboratory mouse strains, including 22L, Me7, RML (Mahal et al., 2007). These cell lines were also used to discriminate between different mouse prion strains in the standard Scrapie cell assay (SSCA) due to the ex vivo cell specific tropism of each strain. In addition to differentiated neuronal cell lines, it was shown that neural stem cells from transgenic mice can also propagate prion infectivity (Milhavet et al., 2006). In addition, the neuronal cell line HpL3-4, established from $\mathrm{PrP}^{0 / 0}$ mice, was demonstrated to be permissive for Scrapie 22L infection when overexpressing murine $\operatorname{PrP}^{c}$ (Maas et al., 2007).

The first transmission of a primary agent, that was not previously adapted to rodents, was achieved in 2001 when sheep Scrapie was inoculated in transgenic RK13 cells, overexpressing a highly permissive variant of ovine PrP (Vilette et al., 2001). In addition glial cell lines (Archer et al., 2004), primary neurons and astrocytes (Cronier, Laude, and Peyrin, 2004) from ovine PrP transgenic mice were demonstrated to be also susceptible to primary sheep scrapie agents. Transmission of chronic wasting disease to a mule deer cell line was also shown (Raymond et al., 2006). Interestingly, the cell metabolism of some cell lines has the capacity to process and degrade prion aggregates (Luhr et al., 2004b; Yadavalli et al., 2004). Exposure to anti PrP antibodies can stop accumulation, replication and propagation of PrPres (Enari, Flechsig, and Weissmann, 2001; Feraudet et 
al., 2005; Peretz et al., 2001). Degradation of PrPres was also modulated by protease inhibitors (Luhr et al., 2004a; Luhr et al., 2004b), which suggests a disturbance of the proteasome degradation system in TSEs (Nunziante et al., 2011). Cell culture models identified heparan sulphates (Ben-Zaken et al., 2003) and the laminin receptor (Gauczynski et al., 2001; Leucht et al., 2003; Rieger et al., 1997) to be involved in the biogenesis of prions, although the underlying molecular mechanism remains elusive. Additionally the spread of infectivity has been demonstrated in cell cultures. Prions have been found hijacking the surface of exosomes (Fevrier et al., 2004), travelling on tunnelling nanotubes between neuronal cells (Gousset et al., 2009) and in cell-free preparations (Leblanc et al., 2006). To date, the neuronal cell death observed in vivo could not be simulated in cell culture systems due to the absence of any obvious cytopathic effects in permanently infected cells, although neuronal apoptosis has been shown in primary Scrapie infected cells (Cronier, Laude, and Peyrin, 2004). By the establishment of transgenic PrP expression in cell culture models, it has been possible to investigate the PrP genotype of different species for interspecies transmissibility. For example, it was shown that only the highly permissive ovine VRQ genotype is susceptible to infection with primary Scrapie inoculum (Sabuncu et al., 2003). The exchange of amino acid at helix 2 and 3 in the mouse PrP dramatically impaired the infection with the Scrapie 22L strain (Maas et al., 2007) in HpL3-4 cells. These studies imply that reverse genetic approaches in cell culture are useful in assessing prion multiplication and could also be used for large scale drug screening approaches (Kocisko et al., 2003). The identification of amphotericin B (Mange et al., 2000) and the polyanion pentosan polysuphate (Caughey and Raymond, 1993) indicats that the systems could also be used to identify antiprion drugs. However, treatment of TSE-infected rodents shows only a delay of disease onset and does not affect the mortality rate (Pocchiari, Schmittinger, and Masullo, 1987). Heparan sulphate mimetics (Adjou et al., 2003; Schonberger et al., 2003), phosphorothioate oligonucleotides (Kocisko et al., 2006), congo red (Caughey and Race, 1992) and active immunisation (Enari, Flechsig, and Weissmann, 2001; Peretz et al., 2001) with anti-prion antibodies are effective in cell culture, but do not cure prion-infected rodents.

Cell culture models have also been used for the titration of the infectivity of tissue. Bioassays have been widely utilised to estimate the infectivity of an analysed tissue. 
These assays are expensive, time-consuming and require a certain number of animals for statistical validation. The establishment of alternative cell culture models to titrate infectivity had been impaired for years due to a low sensitivity. Since the selection of highly permissive cell lines, e.g. N2a subclones (Bosque and Prusiner, 2000), in combination with ultrasensitive PrPres detection methods, cell models have become suitable for the titration of infectious agents. As shown by the group of Charles Weissmann, the standard scrapie cell assay (SSCA) is almost as sensitive as the mouse bioassay (Klohn et al., 2003). An important limitation was drawn for the SSCA regarding its non-permissiveness for naturally occurring prion strains. Although the infection of cell lines has been a powerful tool to study the biology of PrPres, the molecular and cellular reason for the propagation ability of prions remains unclear and impairs the development of cell culture models that are permissive for primary prion agents.

Using transgenic techniques in cell lines, which were previously shown to be permissive, can help to investigate the interspecies transmission of prion diseases. Unfortunately, transient transgenic expression cell systems may not provide constant expression of PrP for the experimental observation periods of several weeks post inoculation. As a result, there is a necessity for stable transgenic PrP expressing cell lines to get closer to the in vivo TSE situation and to unravel the interacting factors, which influence the conversion and aggregation of PrPres. This would further allow systematic analysis of the zoonotic potential of TSEs. Although eradication programs have been established for prion diseases in animals, TSEs remain a subtle but permanent public health issue mainly in captive, but also in wild animals. The transmission capability of TSEs, especially of CWD, needs to be further investigated to clarify the zoonotic potential and species barriers of this prion disease. Transgenic in vitro cell assays would be useful to assist in vivo experiments analysing the interspecies barrier of CWD. So far, the transmissibility of CWD to humans cannot be excluded, mainly due to insufficient transmission data in nonhuman primates. 


\subsection{Outline of the thesis}

This thesis was accomplished within the study "Comprehensive risk assessment of chronic wasting disease (CWD) transmission to non-human primates". The aim of this thesis was to develop a cell culture assay, which could be used to simulate the interspecies transmission of CWD. This was done to assist an in vivo CWD inoculation study in cynomolgus macaques as a non-human primate model for humans. Permissive cell lines were produced by retroviral transduction overexpressing the cellular PrP of a certain species. The species were chosen with regard to the interspecies transmission of CWD to humans. The produced cell lines were characterised for $\mathrm{PrP}^{\mathrm{c}}$ transgene integration in the host cell line, PrPc transgene transcription, PrP expression and surface abundance of $\mathrm{PrP}^{\mathrm{c}}$ as a prerequisite for infection. Once overexpression of transgenic $\mathrm{PrP}$ was confirmed, the cell lines were inoculated with prion-infectious agents. The proof of principle was tested by inoculation with mouse-passaged Scrapie infectious agents. Permissive cell lines were finally used for inoculation with primary CWD-infectious agents to estimate the zoonotic potential of CWD in vitro. 


\section{Material and Methods}

\subsection{Material}

\subsubsection{Consumables and Equipment}

Reaction tubes, pipette tips and laboratory glassware were autoclaved prior to use. Only sterile disposable consumables were used in cell culture.

Table 1.1: General consumables in laboratory work

\begin{tabular}{ll}
\hline name & manufacturer \\
\hline MicroAmp optical 96-well reaction plate & Applied Biosystems \\
MicroAmp optical reaction tubes and caps & Applied Biosystems \\
pistil & Biozym \\
scalpel (carbon steel, \#10, \#15) & B. Braun \\
parafilm M & Carl Roth \\
drigalski spatula & Carl Roth \\
reaction tube $(1.5 \mathrm{ml}, 2 \mathrm{ml})$ & Eppendorf \\
pipettor & Hirschmann \\
centrifugation tube $(15 \mathrm{ml}, 50 \mathrm{ml})$ & Greiner Bio-One \\
agar dishes $(100 \mathrm{~mm})$ & Sarstedt \\
pipette tips RNase/DNase free $(10 \mu \mathrm{l}, 100 \mu \mathrm{l}, 200 \mu \mathrm{l}, 1000 \mu \mathrm{l})$, & Sarstedt \\
reaction tube $(0.2 \mathrm{ml}, 0.5 \mathrm{ml}, 1.5 \mathrm{ml})$ & Sarstedt
\end{tabular}

Table 2.2: General laboratory equipment

\begin{tabular}{ll}
\hline name & manufacturer \\
\hline biological safety cabin HeraSafeKSP & ThermoScientific \\
Real-Time PCR System 7500 & Applied Biosystems \\
LSRII (multi-laser flow cytometer) & BD bioscience \\
thermal cycler My cycler & Bio-Rad \\
centrifuge $5415 \mathrm{R}, 5417 \mathrm{R}, 5424$, concentrator plus & Eppendorf \\
thermo mixer 5436 & Eppendorf \\
pipettes $(1-10 \mu \mathrm{l}, 10-100 \mu \mathrm{l}, 2-200 \mu \mathrm{l}, 100-1000 \mu \mathrm{l})$ & Eppendorf
\end{tabular}


centrifuge $3 \mathrm{~S}-\mathrm{R}$

chemiluminescence imager

shaker Multi Bio 3D, Mini Rocker MR-1

microbiological safety cabinet

$-85^{\circ} \mathrm{C}$ freezer UltraLow U57085

incubator series 5400

microscope IX70 with CCD device and fluorescence imaging unit

mixer VTX-3000L

spectrophotometer Nanodrop

power supply

agarose gel electrophoresis system PerfectBlue

$-20^{\circ} \mathrm{C}$ freezer, $4^{\circ} \mathrm{C}$ fridge

centrifuge Sorvall discovery 90 with rotor TFT 80.4
Heraeus

Intas

Kisker

KOJAIR Ltd

Labotect

NAPCO

Olympus

Omnilab

PEQLAB

PEQLAB

PEQLAB

Privileg

ThermoScientific

Table 2.3: General consumables and components for cell culture

\begin{tabular}{ll}
\hline name & manufacturer \\
\hline dishes (60 mm, 353004; $100 \mathrm{~mm}, 353003)$ & BD Falcon \\
multi well plates (12-well, 353043; 24-well, & BD Falcon \\
353047) & \\
multi well plate (6-well plate 657160) & Carl Roth \\
freezing device "Mr. Frosty” & \\
Neubauer counting chamber & Carl Roth \\
water bath & GFL \\
D-MEM GlutaMAX (61965) & Gibco (Invitrogen life technologies) \\
D-MEM/F-12 GlutaMAX (31331 & Gibco \\
DPBS & Gibco \\
MEM non essential amino acids (10 mM, 11140) & Gibco \\
trypsin, trypsin/EDTA 0.25\% & Gibco \\
tryple select & Invitrogen life technologies \\
cell scraper & Kisker \\
syringe (10 ml) & Norm-Ject \\
cryoTubes (1.8 ml) & NUNC (ThermoScientific) \\
accutase & PAA \\
&
\end{tabular}


penicillin/streptomycin PAA

fetal calf serum, batch numbers: A10106-1008 PAA

(Ref), A10109-0927 (1), A10110-2432 (2),

A10109-2381 (3), A10110-1631 (4), A10110-

3055 (5), A10110-2732 (6), A 10110-1773 (7),

A16010-3199 (8)

flasks $\left(250 \mathrm{~mm}^{2}, 750 \mathrm{~mm}^{2}, 1750 \mathrm{~mm}^{2}\right) \quad$ Sarstedt

serological pipette $(2 \mathrm{ml}, 5 \mathrm{ml}, 10 \mathrm{ml}, 25 \mathrm{ml}) \quad$ Sarstedt

cover slips ThermoScientific

filter $(0.45 \mu \mathrm{m}) \quad$ Sarstedt

\subsubsection{Protein biology}

Unless stated otherwise, all consumables were purchased from Invitrogen life technologies. Protein separation and blotting was performed using the XCell SureLock Mini-Cell and XCell Blot module (Invitrogen life technologies).

Table 2.4: General consumables for protein biology

\begin{tabular}{ll}
\hline name & further information \\
\hline development folder & Applied Biosystems \\
iBlock protein-based blocking reagent & Applied Biosystems \\
$5 \mathrm{ml}$ polysterol tube & BD Falcon \\
ultracentrifugation tube, PA thinwall 4.4 ml & Kisker \\
Immobilon-P transfer membran PVDF & Millipore \\
NuPAGE MOPS SDS running buffer (20x) & diluted in $\mathrm{DI}_{2} \mathrm{O}$ \\
NuPAGE transfer buffer (20x) & diluted in DI $\mathrm{H}_{2} \mathrm{O}, 10 \%$ methanol \\
NuPAGE 12 \% Bis-Tris precast gel, 12-well & \\
LDS sample buffer (4x) & \\
\hline
\end{tabular}




\subsubsection{Chemicals}

Unless stated otherwise, all chemicals were purchased from Carl Roth (Karlsruhe, Germany).

Table 2.5: Chemicals

\begin{tabular}{ll}
\hline name & manufacturer \\
\hline dimethyl sulphoxide & AppliChem \\
ethidium bromide (diluted in $\mathrm{NF} \mathrm{H}_{2} \mathrm{O}$ ) & AppliChem \\
ampicillin (diluted in $\mathrm{NF} \mathrm{H}_{2} \mathrm{O}$ ) & Boehringer \\
puromycin (diluted in $\mathrm{NF} \mathrm{H}_{2} \mathrm{O}$ ) & Invivogen \\
$\mathrm{NF}_{2} \mathrm{O}$ & Qiagen \\
pefabloc (diluted in $\mathrm{NF} \mathrm{H}_{2} \mathrm{O}$ ) & Roche Diagnostic \\
polybrene (diluted in $\mathrm{NF} \mathrm{H}_{2} \mathrm{O}, 107689$ ) & Sigma-Aldrich \\
DEAE-dextran (diluted in $\left.\mathrm{NF} \mathrm{H}_{2} \mathrm{O}, \mathrm{D} 9885\right)$ & Sigma-Aldrich \\
formaldehyde solution $37 \%$ (diluted in PBS) & Sigma-Aldrich \\
$\mathrm{N}$-laurylsarcosine sodium salt (diluted in DI $\mathrm{H}_{2} \mathrm{O}$ ) & Sigma-Aldrich \\
trypan blue (diluted in DPBS) & Sigma-Aldrich \\
Tween-20 & Sigma-Aldrich \\
\hline
\end{tabular}

\subsubsection{Length Markers}

Ladders for length estimation were purchased from Fermentas (ThermoScientific).

\section{Table 2.6: DNA and protein ladders}

name

GeneRuler 1kb plus DNA ladder

GeneRuler low range DNA ladder

Spectra multicolour broad range protein ladder 


\subsubsection{Enzymes}

Unless stated otherwise, enzymes were purchased from Fermentas (ThermoScientific).

Table 2.7: Enzymes

\begin{tabular}{ll}
\hline name & concentration \\
\hline BamHI restriction enzyme & $10 \mathrm{U} / \mu \mathrm{l}$ \\
EcoRI restriction enzyme & $10 \mathrm{U} / \mu \mathrm{l}$ \\
SalI restriction enzyme & $10 \mathrm{U} / \mu \mathrm{l}$ \\
DNase I (Applichem) & Aliquoted à $10 \mathrm{mg} / \mathrm{ml}$ \\
DNase I (Fermentas) & $1 \mathrm{U} / \mu \mathrm{l}$ \\
Phusion High Fidelity DNA Polymerase (Finnzymes) & $2 \mathrm{U} / \mu \mathrm{l}$ \\
Proteinase K (\# 1092766, Boehringer) & Aliquoted à $10 \mathrm{mg} / \mathrm{ml}$ \\
Reverse transcriptase (Bioscript, Bioline) & $200 \mathrm{U} / \mu \mathrm{l}$ \\
RiboLock RNAse inhibitor & $40 \mathrm{U} / \mu \mathrm{l}$ \\
T4 DNA Ligase & $5 \mathrm{U} / \mu \mathrm{l}$ \\
\hline
\end{tabular}

\subsubsection{Reaction components and commercial kits}

Unless stated otherwise, the technical procedure was performed according to the manufacturers' instructions.

Table 2.8: Reaction components and commercial kits

\begin{tabular}{ll}
\hline name & manufacturer \\
\hline innuPrep DNA & analytikJena Life Science \\
BigDye Cycle Sequencing Kit & Applied Biosystems \\
Power SYBR-green PCR Master Mix & Applied Biosystems \\
$\alpha$-select competent cells gold & Bioline \\
CloneJET1.2 PCR Cloning Kit & Fermentas \\
dNTPs (10 mM each) & Fermentas \\
Loading dye (6x) & Fermentas \\
MgCl $_{2}(10 \mathrm{mM})$ & Fermentas \\
TRIzol & Invitrogen life technologies \\
Lipofectamin2000 & Invitrogen life technologies
\end{tabular}


NucleoSpin Plasmid

PAXgene blood RNA kit and tube

QIAshredder

RNAeasy plus mini

MinElute gel extraction kit

EndoFree plasmid maxi kit

CDP-Star, ready-to-use
Macherey-Nagel

Preanalytix

Qiagen

Qiagen

Qiagen

Qiagen

Roche 


\subsubsection{Antibodies for protein detection}

Monoclonal antibodies were used for immunodetection by Western blot (WB) analysis or flow cytometry.

Table 2.9: Antibodies with corresponding reference and application

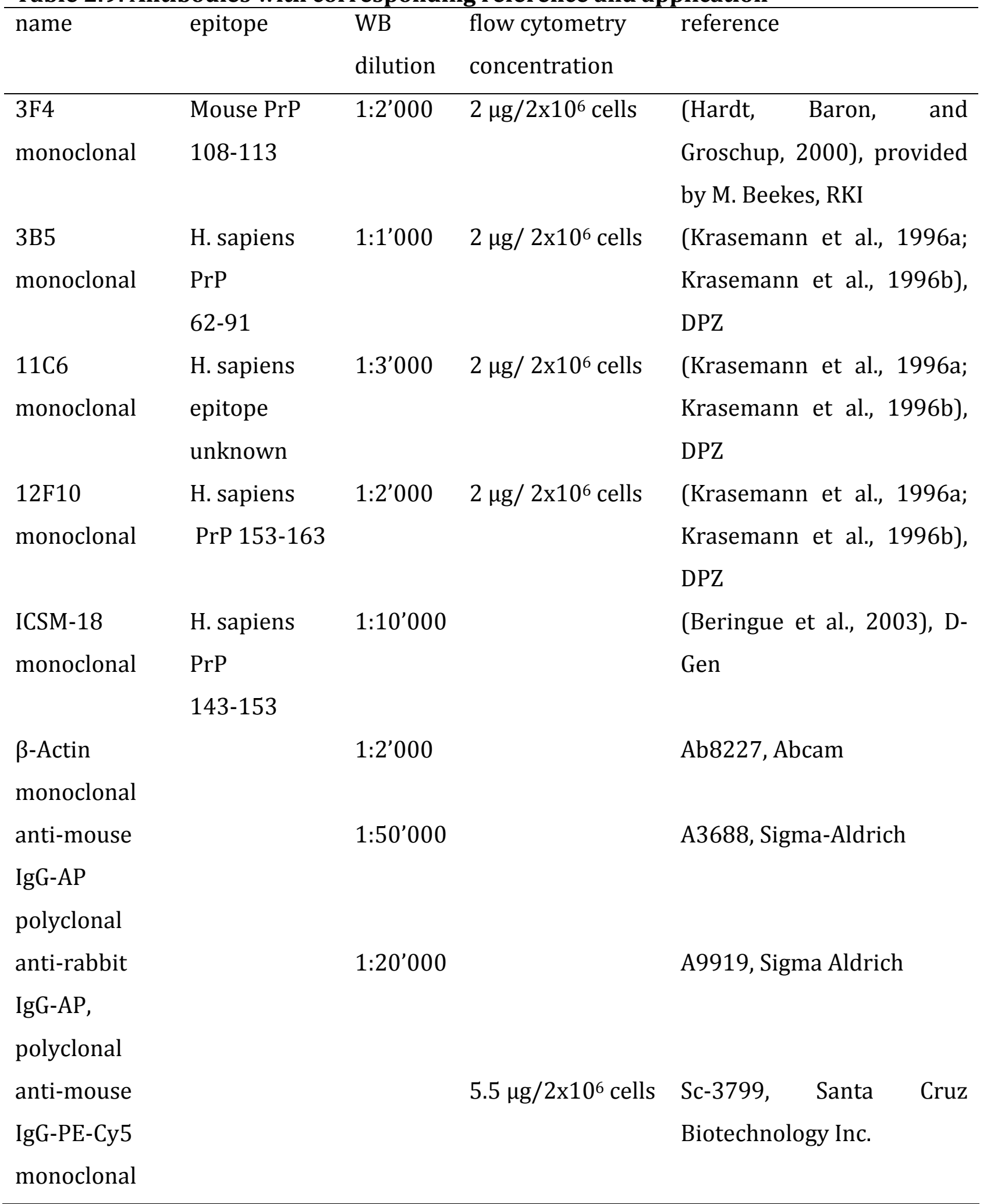


Table 2.10: Culture medium and buffers

name composition

293FT culture medium

$10 \%(\mathrm{v} / \mathrm{v})$ FCS, $1 \%(\mathrm{v} / \mathrm{v})$ penicillin $\left(10^{\prime} 000 \mathrm{U} / \mathrm{ml}\right)$ and streptomycin (10 mg/ml), $1 \%$ (v/v) MEM non-essential amino acids (10 $\mathrm{mM}$ ) filled to $500 \mathrm{ml}$ D-MEM

HpL3-4 culture medium $10 \%(\mathrm{v} / \mathrm{v})$ FCS, $1 \%(\mathrm{v} / \mathrm{v})$ penicillin $\left(10^{\prime} 000 \mathrm{U} / \mathrm{ml}\right)$ and streptomycin (10 mg/ml) filled to $500 \mathrm{ml} \mathrm{D-MEM}$

SH-SY5Y culture medium $15 \%$ (v/v) FCS, $1 \%(\mathrm{v} / \mathrm{v})$ penicillin $\left(10^{\prime} 000 \mathrm{U} / \mathrm{ml}\right)$ and streptomycin $(10 \mathrm{mg} / \mathrm{ml})$ filled to $500 \mathrm{ml}$ D-MEM/F-12

freezing medium $20 \%(\mathrm{v} / \mathrm{v})$ FCS, $10 \%(\mathrm{v} / \mathrm{v})$ DMSO, $80 \%$ (v/v) culture medium of respective cell line

LB-medium $1 \%(\mathrm{w} / \mathrm{v})$ tryptone/peptone, $1 \%(\mathrm{w} / \mathrm{v})$ yeast extract, 0.5 $\%(\mathrm{w} / \mathrm{v})$ sodium chloride (Bertani, 1951)

LB-Amp medium LB-medium supplemented with $100 \mu \mathrm{g} / \mathrm{ml}$ ampicillin

LB-Amp agar

PBS LB-Amp medium supplemented with $1.5 \%$ (w/v) agar $120 \mathrm{mM} \mathrm{NaCl}, 17 \mathrm{mM} \mathrm{Na}_{2} \mathrm{HPO}_{4}, 3 \mathrm{mM} \mathrm{KH}_{2} \mathrm{PO}_{4}$ in $\mathrm{DI} \mathrm{H}_{2} \mathrm{O}$, $\mathrm{pH} 7.2$

TBS 100 mM NaCl, 10 mM Tris-HCl in DI $\mathrm{H}_{2} \mathrm{O}$, pH 7.6

TBS-T TBS supplemented with $0.1 \%(\mathrm{v} / \mathrm{v})$ Tween-20

TAE (10x) $400 \mathrm{mM}$ Tris-HCl, $10 \mathrm{mM}$ EDTA, $200 \mathrm{mM}$ acetic acid in DI

stripping buffer $\mathrm{H}_{2} \mathrm{O}, \mathrm{pH} 8.0$ $200 \mathrm{mM}$ glycine, $3.46 \mathrm{mM}$ SDS, $1 \%$ Tween20 in DI $\mathrm{H}_{2} \mathrm{O}$, $\mathrm{pH} 2.2$ 


\subsubsection{Oligonucleotides for PCR}

All oligonucleotides used were delivered HPLC-purified from TIB Molbiol. Random hexamers for cDNA synthesis were purchased from Fermentas (ThermoScientific).

Table 2.11: Oligonucleotides for amplification

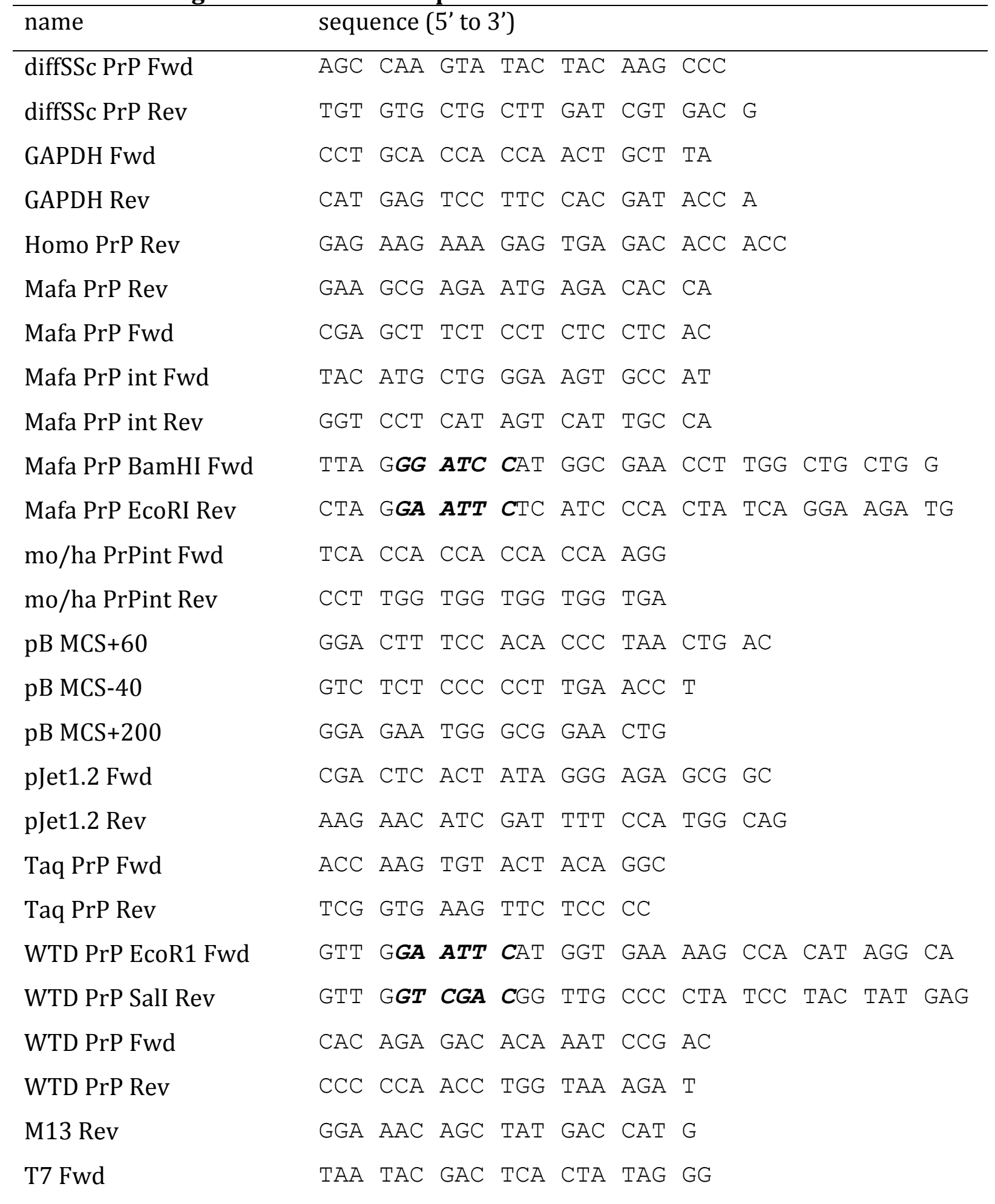

Restriction cleavage sites are italic 


\subsubsection{Plasmids}

Vectors were used for different purposes. The pJet1.2 vector was used for subcloning of PCR products. The pVPack vector system (Stratagene) was used for the generation of retroviral particles. Both are coding for viral proteins, which are necessary to generate viral particles. The pVPack-GP expression vector carries the gag-pol genes and encodes for the trans-acting internal structural proteins, the reverse transcriptase (RT) and integrase. A CMV promoter drives the expression of the gag-pol elements from the pVPack-GP vector and the env elements from the pVPack-VSV-G. They also contain an internal ribosome entry site (IRES) linked to a downstream drug-resistance cassette that enables selection. Additionally to the pVPack packaging vectors, the retroviral expression vector pBABE was used for the expression of transgenic PrP. It is derived from the moloney murine leukemia virus (MMLV) and carries the viral cis-acting RNA packaging signal $\psi^{+}$(psi) which is required for the viral RNA to be packaged into assembled particles by interaction with gag (Clever, Sassetti, and Parslow, 1995) and env (Narayanan and Makino, 2001). Other cis-acting elements encoded on the plasmid are the long terminal repeats (LTR's). Furthermore, it contains a bacterial origin of replication, a multiple cloning site (MCS) for the insertion of a transgene, and ampicillin and puromycin resistance genes as selection markers. As a visual reporter for transfection and transduction, respectively, pBABE-EGFP was used. It encodes for the enhanced green fluorescent protein. The green fluorescent protein, originally purified from Aequorea victoria, is a protein that emits light at a wavelength of $395 \mathrm{~nm}$ (major excitation peak) if excited by blue light (Prasher et al., 1992; Prendergast and Mann, 1978; Shimomura, Johnson, and Saiga, 1962). The enhanced GFP has single mutations in the active core of the protein and therefore emits light at a wavelength of $488 \mathrm{~nm}$, which could be used to visualize transfected or transduced cells, respectively.

Table 2.12: Plasmids

\begin{tabular}{ll}
\hline name & manufacturer \\
\hline pJet1.2 & Fermentas \\
pGEM-Teasy-GAPDH & Promega, kindly provided by Dipl. Biol. Wiebke Ibing \\
pVPack-GP, pVPack-VSV-G & Stratagene \\
pBABE-Puro & Cellbiolabs \\
pBABE-EGFP & Cellbiolabs \\
\hline
\end{tabular}




\subsection{Methods}

\subsubsection{Cell biology}

\subsubsection{Cell culture cultivation}

Unless stated otherwise, cells were grown subconfluent in their respective culture medium (see table 2.10) preferentially in a $75 \mathrm{~cm}^{2}$ cell culture flask. Cells were incubated in a $5 \% \mathrm{CO}_{2}$ atmosphere and 95-100\% humidity. All liquids used in cell culture were sterile and equilibrated to $37^{\circ} \mathrm{C}$ in a water bath prior to use. Cells were passaged when they reached the respective confluency (see table 2.13). Therefore, culture medium was removed; monolayers were rinsed with DPBS and detached with trypsin/EDTA for approximately 3'. Trypsin was inactivated by addition of $4 \mathrm{x}$ volume of FCS-containing culture medium. Depending on the cell line, $1 / 3-1 / 6$ of the cells were transferred to a new flask and covered with up to $20 \mathrm{ml}$ culture medium.

Table 2.13: Cell lines with corresponding reference and culture conditions

\begin{tabular}{lllll}
\hline cell line & confluency & trypsin & dilution & reference \\
\hline HEK293FT, & $90 \%$ & $0.25 \%$ & $1: 3$ & (Graham et al., 1977), \\
subclone from human & & (without & & purchased from Invitrogen \\
embryonic kidney cells & & EDTA) & & life technologies \\
HpL3-4, & $95 \%$ & $0.25 \%$ & $1: 5$ & (Kuwahara et al., 1999), \\
hippocampus neurons & & & & kindly provided by Prof. T. \\
from PrPo/0 mice & & & & Onodera \\
tg(mo)PrP HpL3-4, & $95 \%$ & $0.25 \%$ & $1: 5$ & kindly provided by Prof. I. \\
HpL3-4 cells expressing & & & & Vorberg (DZNE Bonn) \\
transgenic mouse PrP & & & & \\
SH-SY5Y, & & & & (Biedler, Helson, and \\
subclone from human & & $0.125 \%$ & $2: 3$ & Spengler, 1973; Biedler et \\
neuroblastoma cell line & & & & al., 1978), DSMZ \\
SK-N-SH & & & &
\end{tabular}




\subsubsection{Determination of cell density}

An aliquot of the cell suspension was diluted $1 / 10$ in a total volume of $100 \mu$ l of PBS supplemented with $0.05 \%(\mathrm{w} / \mathrm{v})$ trypan blue. $10 \mu \mathrm{l}$ of this suspension was applied to a Neubauer counting chamber and cell density was calculated excluding the stained cells using the below stated formula.

$\frac{\# \text { cells }}{\text { \#squares }} \cdot$ dilutionfactor $\cdot 10^{4}=$ cells $/ \mathrm{ml}$

\subsubsection{Freezing of cells}

For later use, cells were expanded to an adequate amount and vitally deep frozen. Cells were harvested by trypsin detachment. The cell suspension was pelleted by centrifugation at 700xg for 7' at RT. The pellet was resuspended in freezing medium (see table 2.10 ), adjusted to a density of $5 \times 10^{6}$ cells $/ \mathrm{ml}$ and aliquoted in cryo vials. The vials were placed in a cryo-freezing device filled with isopropyl alcohol to ensure slow cooling with $-1^{\circ} \mathrm{C} / \mathrm{min}$. After $24 \mathrm{~h}$ at $-80^{\circ} \mathrm{C}$, vials were transferred to liquid nitrogen for longterm storage.

\subsubsection{Revitalizing of cells}

After 40 cycles of passaging, cells were removed from the permanent cell culture and a new aliquot of the respective cell line was thawed. One cryo vial was removed from liquid nitrogen and quickly thawed in a $37^{\circ} \mathrm{C}$ warmed water bath. The warmed cell suspension was transferred to a $50 \mathrm{ml}$ falcon adding $40 \mathrm{ml}$ culture medium. Cells were sedimented at 700xg for 7' at RT. The pellet was resuspended in $20 \mathrm{ml}$ culture medium and transferred to a $75 \mathrm{~cm}^{2}$ flask. Cells were passaged for at least $7 \mathrm{~d}$ prior to use.

\subsubsection{Acute antibiotic toxicity test}

Acute toxicity of puromycin to cultured cells was measured. The puromycin gene encodes for the puromycin $\mathrm{N}$-acetyl-transferase (PAC) and is derived from the 
bacterium Streptomyces alboniger (Vara, Perez-Gonzalez, and Jimenez, 1985). The nucleoside antibiotic inhibits protein synthesis due to premature chain termination at the ribosome entry site during protein translation (Darken, 1964). Additionally, it blocks the protein import into mitochondria by the interference with an intramitochondrial ATP-dependent reaction (Price and Verner, 1993). Cells, which gain the resistance gene for the puromycin, resist the treatment because they can degrade the antibiotic. $10^{5}$ HpL3-4 or 2x105 SH-SY5Y cells, respectively, were seeded in a 12-well plate and incubated for $24 \mathrm{~h}$. The following day, culture medium was removed and fresh culture medium was added including puromycin in concentrations ranging from 0-10 $\mu \mathrm{g} / \mathrm{ml}$. Cells were incubated for the next 5 consecutive days, with medium exchange at day 2 and 4. Images of cells were taken at day 2, 3 and 5.

\subsubsection{Infection of cells}

In order to infect cells with prions, transgenic cell lines were inoculated with infectious brain or cell homogenate. $4 \times 10^{5}$ transgenic HpL3-4 cells or $4 \times 10^{5}$ transgenic SH-SY5Y cells, respectively, were seeded in triplicates in 6-well plates and grown to 60-70\% confluence. Prior inoculation, cells were washed with DPBS. Cell homogenate and brain homogenate was diluted in the respective cell culture medium. Then, target cells were inoculated with $5 \times 10^{6}$ cells or $1 \%$ brain homogenate in a total volume of $500 \mu \mathrm{l}$ for $6 \mathrm{~h}$. Subsequently $500 \mu \mathrm{l}$ of culture medium-homogenate mix was added to each well. After $6 \mathrm{~h} 3 \mathrm{ml}$ cell culture medium was added to each well. Cells were passaged at a confluence of $90 \%$ (HpL3-4) or $80 \%$ (SH-SY5Y) according to the cultivation procedure of the respective cell line (see table 2.13). An additional aliquot was plated in $60 \mathrm{~mm}$ dishes after 9, 16, 23 and 40 days post infection (dpi) and grown to confluency.

\subsubsection{Generation of retroviral particles}

\subsection{Transfection of cells}

Transfection in molecular biology is a technique used to introduce nucleic acids to eukaryotic cells. Beside many other reagents, cells can be transfected by lipofection (Felgner et al., 1987). DNA is included into small cationic vesicles, which comprise the similar phospholipid bilayer structure as compared to the cell membrane. The 
liposomes then fuse with the cell membrane thereby transferring the plasmid DNA into the cytoplasm.

For transfection experiments, 3x106 HEK 293FT cells were plated in $10 \mathrm{~cm}$ dishes in culture medium without addition of penicillin/streptomycin. Cells were cultivated for 24 $\mathrm{h}$ to reach a confluency of maximal $60-70 \% .5 \mu \mathrm{g}$ of pVPack-GP, pVPack-VSV-G and pBABE-puro plasmids were prepared in a total volume of $100 \mu \mathrm{l}$ Opti-MEM. $22.5 \mu \mathrm{l}$ of Lipofectamin 2000 was diluted in a $100 \mu \mathrm{l}$ Opti-MEM and incubated according to the manufacturer's instructions. Plasmid DNA and transfection reagent was mixed and incubated for 20' at RT. Meanwhile, culture medium was removed from the 293FT cells and replaced by $3 \mathrm{ml}$ Opti-MEM (without addition of FCS and antibiotics). DNA/transfection mix was added to the HEK 293 FT cells, removed after $5 \mathrm{~h}$ of incubation at $37^{\circ} \mathrm{C}$ and replaced by culture medium. Successful transfection was monitored after $24 \mathrm{~h}$ by EGFP expression when pBABE-EGFP was used in a control experiment.

\subsection{Harvest of retroviral particles}

Once viral RNA is encapsulated, viral particles can bud off from the packaging cells and are released to the supernatant. The supernatant of transiently transfected cells was collected and used to transduce target cells.

Prior to the harvest of viral particles, HEK 293 FT cells were cotransfected with pVPackGP, pVPack-VSV-G and pBABE-puro (or pBABE-EGFP as internal control). Twenty-four hours after cotransfection, culture medium was replaced. After $48 \mathrm{~h}$ of incubation, $10 \mathrm{ml}$ supernatant including the viral particles was harvested and collected in a $15 \mathrm{ml}$ tube. To remove cell debris, supernatant was passed through a sterile $0.45 \mu \mathrm{m}$ filter using a sterile $10 \mathrm{ml}$ syringe, and directly used or stored in aliquots at $-80^{\circ} \mathrm{C}$.

\subsection{Retroviral transduction}

Transduction in molecular biology is a technique to transfer nonviral genetic material to a target cell via a viral particle, that delivers the genetic material (Miller, 1997) into the recipient cell. This method requires the production of intact viral particles by a virus packaging cell line (see above). For successful assembly of viral particles, cells need to express viral genes coding for gag- pol and env. Upon transduction as gene transfer, viral RNA that also encodes for the transgene is flanked by long terminal repeats (LTRs), 
which enable stable integration into the host genome (Shoemaker et al., 1981). These retroviral elements flank functional genes and mediate the insertion of viral DNA into host genomes. Due to the arrangement of viral genes on different plasmids, the particles are only capable to infect a target cell once and are not able to replicate or propagate intact virus. An antibiotic resistant gene on the expression vector enables selection for positively transduced cells.

Two different protocols were tested for their transduction efficiency. In the first setup, $10^{5}$ target cells were seeded in 6-well plates and incubated for $1 \mathrm{~d}$. At the following day, $1 \mathrm{ml}$ of retroviral supernatant was mixed with $10 \mu \mathrm{g} / \mathrm{ml}$ DEAE-dextran and transferred to the monolayer of the target cells. The viral particles were incubated on the cells for $3 \mathrm{~h}$ at $37^{\circ} \mathrm{C}$. After the incubation time, the viral supernatant was removed and fresh culture medium was added to the cells. For the second setup, later referred to as spinduction, target cells were detached by trypsinisation. Two $\mathrm{ml}$ of retroviral supernatant was transferred to a $50 \mathrm{ml}$ tube and polybrene was added to a final concentration of $3 \mu \mathrm{g} / \mathrm{ml} .10^{5} \mathrm{HpL} 3-4$ cells/500 $\mu \mathrm{l}$ culture medium or $2 \times 10^{5} \mathrm{SH}-\mathrm{SY} 5 Y$ cells $/ 500 \mu \mathrm{l}$ culture medium, respectively, were transferred to the retroviral supernatant. The mixture was sedimented at $1500 x \mathrm{xg}$ for $3 \mathrm{~h} 30^{\prime}$ at $32^{\circ} \mathrm{C}$ (Suter et al., 2006). After centrifugation, $3 \mathrm{ml}$ of culture medium was added; the remaining pellet was resuspended vigorously and subsequently transferred to a 6-well plate. Puromycin treatment was accomplished $72 \mathrm{~h}$ after transduction for $7 \mathrm{~d}$ to select for successfully transduced cells.

\subsubsection{Molecular Biology}

\subsubsection{Isolation of ribonucleic acids (RNA)}

Total RNA can be isolated from biological samples in multiple ways. Two different isolation methods were carried out according to the quality requirements of the downstream applications. Isolation of RNA from cultured cells was accomplished using a spin-column based method. RNA binds selectively to the silica membrane of the column depending on $\mathrm{pH}$ and salt conditions and can then be eluted from the membrane after stringent washing steps. Isolation of RNA from tissues was accomplished using the trizol reagent. It is based on a phenol-chloroform extraction combined with a protein denaturation step by the use of guanidine thiocyanate (Chomczynski, 1993; Chomczynski and Sacchi, 1987). It relies on a phase separation of an aqueous phase 
(that contains the RNA) and an organic phase (that contains DNA and proteins) by centrifugation. RNA is then precipitated from the aqueous phase with isopropyl alcohol.

\subsection{Isolation of RNA from blood}

For isolation of RNA, blood from M. fascicularis was collected in a collection tube provided with the PAXgene blood RNA kit and incubated for $2 \mathrm{~h}$ at RT to ensure total lyses of blood cells. Further processing was accomplished according to the manufacturer's instructions. The quantity of purified RNA was assessed by spectrophotometry and RNA sample was stored at $-80^{\circ} \mathrm{C}$.

\subsection{Isolation of RNA from cultured cells}

For isolation of RNA, cells were harvested by trysinisation and cell density was determined. RNA was isolated from $5 \times 10^{6}$ cells using the RNeasy Plus kit according to the manufacturer's instruction. In order to disrupt cells, an additional homogenization step was accomplished by using Qiashredder columns. To ensure total removal of genomic DNA, gDNA eliminator columns were used (Qiagen). Isolated RNA was eluted in $30 \mu \mathrm{l}$ nuclease-free water. The quantity of purified RNA was assessed by spectrophotometry. RNA samples were stored at $-80^{\circ} \mathrm{C}$.

\subsection{Isolation of RNA from brain tissue}

For isolation of RNA, pieces of brain tissue were weighted to ensure that the maximal amount of $100 \mathrm{mg}$ was not exceeded. Brain tissue was transferred to a reaction tube and $1 \mathrm{ml}$ trizol reagent was added. The tissue was homogenized using a disposable pistil and incubated for 5' at RT. The sample was centrifuged at $2000 \mathrm{xg}$ for $10^{\prime}$ at $4^{\circ} \mathrm{C}$ and the supernatant was transferred to a new reaction tube. $200 \mu \mathrm{l}$ chloroform was added, gently mixed and incubated for 3' at RT. The sample was centrifuged at $12^{\prime} 000 \mathrm{xg}$ for $15^{\prime}$ at $4^{\circ} \mathrm{C}$. The upper aqueous phase was transferred to a new tube and $500 \mu \mathrm{l}$ isopropyl alcohol was added. The mix was incubated for 10' at RT and subsequently centrifuged at $12,000 \mathrm{xg}$ for $10^{\prime}$ at $4^{\circ} \mathrm{C}$. The supernatant was removed and $1 \mathrm{ml} 75 \%$ ethanol was added to wash the pellet. The sample was centrifuged at $7,500 \mathrm{xg}$ for $5^{\prime}$ at $4^{\circ} \mathrm{C}$. The supernatant was removed completely; the pellet resuspended in $30 \mu \mathrm{l}$ nuclease-free water, and the 
quantity of purified RNA was assessed by spectrophotometry. To reduce gDNA contamination, RNA was digested by DNase I $(\rightarrow)$. RNA samples were stored at $-80^{\circ} \mathrm{C}$.

\subsubsection{Isolation of gDNA from cultured cells}

Isolation of gDNA was accomplished to quantify the integration efficiency of the retroviral expression vectors. The used protocol involves disruption and lysis of the starting material, followed by removal of contaminants such as proteins (proteinase $\mathrm{K}$ digest) and recovery of the DNA. gDNA was extracted from cells using a column-based method, where DNA can be bound and eluted from a silica membrane under high salt and $\mathrm{pH}$ conditions.

For isolation of gDNA cells were harvested by trypsinisation and cell density was determined. Genomic DNA was isolated from $5 \times 10^{6}$ cells using the innuPREP DNA kit according to the manufacturer's instructions. The gDNA was eluted in $100 \mu \mathrm{l}$ nucleasefree water, concentration was determined and samples were stored at $-20^{\circ} \mathrm{C}$.

\subsubsection{DNase I digestion}

DNase I is an endonuclease enzyme that hydrolyzes single and double stranded DNA by cleavage of phosphodiester bonds producing mono- and oligodeoxyribonucleotides with 5'-phosphate and 3'-OH groups.

To reduce contamination of genomic DNA that co-precipitated with the RNA a DNase I digest was performed. $1 \mu \mathrm{g}$ RNA was digested by $1 \mathrm{U}$ DNase I with addition of $40 \mathrm{U}$ RNase inhibitor and $1 \mathrm{x}$ DNase I buffer (including $\mathrm{MgCl}_{2}$ ) in a total volume of $10 \mu \mathrm{l}$. In contrast to the manufacturer's instructions, samples were incubated at $37^{\circ} \mathrm{C}$ for $30^{\prime}$ and DNase was inactivated at $95^{\circ} \mathrm{C}$ for $5^{\prime}$. The DNase I digested RNA samples were subsequently used for cDNA synthesis or stored at $-80^{\circ} \mathrm{C}$ for later use.

\subsubsection{Ethanol/sodium precipitation}

In order to concentrate or desalt nucleic acids precipitation was performed. Nucleic acids form ionic bonds with sodium-acidic acid that precipitate in $96 \%$ ethanol. If the concentration of ethanol decreases (70\%) the sodium-acidic acids are solubilised in the 
aqueous phase while the nucleic acids remain precipitated as long as the temperature is below $0^{\circ} \mathrm{C}$. The purified pellet can then be resolved in $\mathrm{NF}_{2} \mathrm{O}$.

For precipitation $1 / 10 \mathrm{x}(\mathrm{v} / \mathrm{v})$ of $3 \mathrm{M}$ sodium acetate $(\mathrm{pH} 5.5)$ and $2.5 \mathrm{x}(\mathrm{v} / \mathrm{v})$ ethanol (96\%) was added to the samples and incubated for $2 \mathrm{~h}$ at $-20^{\circ} \mathrm{C}$. Afterwards, samples were centrifuged at $16^{\prime} 000 \mathrm{xg}$ for $30^{\prime}$ at $4^{\circ} \mathrm{C}$ and, after removing the supernatants, pellets were washed twice in icecold $70 \%$ ethanol at $16^{\prime} 000 \mathrm{xg}$ for $15^{\prime}$ at $4^{\circ} \mathrm{C}$. The pellets were dried in a SpeedVac at RT and resolved in a suitable volume of $\mathrm{NF}_{2} \mathrm{O}$.

\subsubsection{5 cDNA synthesis}

In order to perform PCR or qPCR on the extracted RNA, cDNA synthesis was accomplished. During cDNA synthesis a single stranded DNA molecule is produced from a single stranded RNA molecule by a reverse transciptase (RT). First a primer anneals to the RNA molecule that produces a short DNA/RNA-hybrid. This hybrid is recognized by the RT and the RNA strand is reversely transcribed.

For reverse transcription of RNA to cDNA, samples contained 100 ng RNA, 10 ng random hexamer primer, $1 \mathrm{x}$ buffer, $2 \mathrm{mM}$ dNTPs, $0.5 \mathrm{U}$ RNase inhibitor and $5 \mathrm{U}$ reverse transcriptase diluted in $\mathrm{NF} \mathrm{H}_{2} \mathrm{O}$ to a final volume of $20 \mu \mathrm{l}$. For denaturation and hybridization, RNA and primer were preincubated for $5^{\prime}$ at $70^{\circ} \mathrm{C}$ followed by $10^{\prime}$ at $4^{\circ} \mathrm{C}$ before the addition of all other components. Elongation and transcription of the template was performed at $42^{\circ} \mathrm{C}$ for $60^{\prime}$. Thereafter, the enzyme was inactivated at $70^{\circ} \mathrm{C}$ for $10^{\prime}$. For each sample a negative control was carried along during the experiments by omission of the reverse transcriptase during cDNA synthesis (-RT control). The cDNA samples were stored at $-20^{\circ} \mathrm{C}$.

\subsubsection{Polymerase chain reaction}

Polymerase chain reaction (PCR) was performed in order to amplify a certain gene product to several orders of magnitude (Mullis et al., 1986) or to quantify the expression of a gene (quantitative PCR). It is based on annealing of two sequence-specific oligonucleotides (sense/antisense primer) to a certain DNA and complementary elongation of the oligonucleotides due to the activity of a thermostable DNA polymerase. Repeating cycles of annealing and elongation then lead to the amplification of the 
fragment. The quantification of the product can be performed by measurement of an intercalating fluorescent dye that binds to the minor groove of dsDNA. The fluorescence intensity increases proportional to the amount of newly synthesised DNA. Specificity of the primers was tested by two different negative controls, i.e. by replacing template DNA by $\mathrm{NF}_{2} \mathrm{O}$, or by a -RT control. Correct fragment size of the PCR products was checked by standard agarose gel electrophoresis.

Amplification of the open reading frame (ORF) of PrP from cDNA (2.2.2.5) for further cloning into vectors was accomplished by PCR using a proof reading DNA polymerase (Phusion DNA Polymerase). Therefore, $1 \mu \mathrm{l}$ cDNA was mixed with $1 \mathrm{x}$ PCR-buffer GC, $1 \mathrm{mM} \mathrm{MgCl}$, $3.2 \mathrm{mM}$ dNTP's, $0.02 \%$ DMSO, $400 \mathrm{nM}$ sense primer, $400 \mathrm{nM}$ antisense primer and $1 \mathrm{U}$ Phusion DNA Polymerase in a final volume of $25 \mu \mathrm{l}$. The initial denaturation step was performed for $3^{\prime}$ at $98^{\circ} \mathrm{C}$, followed by 35 cycles of $10^{\prime \prime}$ at $98^{\circ} \mathrm{C}$ (denaturation), $30^{\prime \prime}$ at $62^{\circ} \mathrm{C}$ (annealing) and $40^{\prime \prime}$ at $72^{\circ} \mathrm{C}$ (elongation). The reaction was terminated by $5^{\prime}$ at $72^{\circ} \mathrm{C}$ followed by an on-hold cooling step at $4^{\circ} \mathrm{C}$. Subsequently, sample was diluted in $1 \mathrm{x}$ loading dye, separated by DNA gel electrophoresis (2.2.2.7) and target fragment was excised for gel extraction (2.2.2.8).

\subsection{Quantitative PCR (qPCR)}

For quantification of PrP in transgenic cells qPCR was performed. Therefore, $1 \mu \mathrm{l}$ cDNA or 100 ng gDNA, respectively, was diluted in 2x Power SYBR green mix including $400 \mathrm{nM}$ sense and antisense primer, respectively, in a final volume of $50 \mu \mathrm{l}$. The sample was then divided in two technical replicates and transferred to an optical 96-well plate. QPCR conditions were the following: $10^{\prime}$ at $95^{\circ} \mathrm{C}$ for polymerase activation, followed by 45 cycles of $15^{\prime \prime}$ at $95^{\circ} \mathrm{C}$ (denaturation) and $45^{\prime \prime}$ at $58^{\circ} \mathrm{C}$ (primer annealing and elongation). The data were collected as fluorescence alterations to a manual threshold cycle $\left(\mathrm{C}_{\mathrm{t}}\right)$ of 0.2. To allow normalization of data, expression of GAPDH was quantified in each sample. Absolute quantification of transgene abundance or expression was assessed by simultaneous measurement of serial dilutions of plasmid DNA $\left(10^{6}-10^{3}\right.$ copies $\left./ \mu \mathrm{l}\right)$ carrying the PrP (pBABE-(mafa)PrP, pBABE-(sai)PrP) insert or a GAPDH fragment (pGEM-Teasy-GAPDH, cloned by Dipl.Biol. Wiebke Ibing). For analyses, the total number of transgenic genomic integrations or mRNA copies per 100 copies GAPDH was calculated (3.3.1). The SDS 7500 system software accomplished the processing of data. The qPCR product was applied to DNA gel electrophoresis (2.2.2.7). 


\subsubsection{Agarose gel electrophoresis for separation of DNA}

Agarose gel electrophoresis is used to separate DNA fragments according to their size in an electric field. In order to visualize the DNA an intercalating agent with a fluorescent tag is commonly used. We have used ethidium bromide that intercalates into doublestranded DNA and is then visualized at $285-320 \mathrm{~nm}$. Gel electrophoresis was used subsequent to PCR, qPCR or restriction enzyme digest.

With respect to the expected length of the product a 1, 1.5 or $2 \%(\mathrm{w} / \mathrm{v})$ agarose gel was prepared. The agarose was dissolved in TAE-buffer and boiled in a microwave oven. For fluorescence detection of DNA $1 \%(\mathrm{v} / \mathrm{v})$ ethidium bromide was added. The liquid was cast into a gel chamber and left for 30' at RT to solidify. The gel was transferred to an electrophoresis chamber filled with TAE-buffer. Samples were diluted in $6 x$ loading dye and applied to the gel slots. A suitable DNA ladder according to the expected fragment size was used for length determination. The separation of DNA was performed at $80 \mathrm{~V}$ for 40-60'. Visualization was performed under UV light at a wavelength of $314 \mathrm{~nm}$. If necessary for downstream applications, DNA fragment was removed from the gel by gel extraction (2.2.2.8).

\subsubsection{Purification of PCR products by gel extraction}

Agarose gel extraction is a molecular technique to isolate a DNA fragment from an agarose matrix. We have used a spin column based extraction that is based on binding of DNA to a silica membrane under low $\mathrm{pH}$ and high chaotropic salt conditions, washing of column to remove salt and elution of DNA in a small volume of $\mathrm{NF}_{2} \mathrm{O}$.

Linearised plasmid DNA and PCR fragments were separated by gel electrophoresis according to their respective size. Target fragments were excised from the agarose gel using a disposable scalpel. The gel fragment was weighted and dissolved in 3x QG buffer (provided with gel extraction kit). The following steps were performed according to the manufacturer's instructions. The purified DNA was eluted in $10 \mu \mathrm{NF} \mathrm{H}_{2} \mathrm{O}$ and concentration was determined. 


\subsubsection{Restriction cleavage}

DNA can be cleaved by hydrolysis due to the activity of a restriction endonuclease (see table 2.7), leaving ds DNA pieces with a ss overhanging end. These ends are "sticky" and can later be ligated by the enzyme DNA ligase (2.2.2.10) to another piece of DNA with a complementary ss overhang.

The purified PCR product or $5 \mu \mathrm{g}$ of the target expression plasmid, respectively, were cleaved by $1 \mu \mathrm{l}$ fast digest restriction endonuclease (see table 2.7) in fast digest green buffer in a final volume of $20 \mu \mathrm{l}$ for $30^{\prime}$ at $37^{\circ} \mathrm{C}$. The enzyme was thermally inactivated for $5^{\prime}$ at $80^{\circ} \mathrm{C}$. For double cleavage, $1 \mu \mathrm{l}$ of the second restriction enzyme was added to the pre-mix after intermediated cooling for 5'. The reaction mix was again incubated for $30^{\prime}$ at $37^{\circ} \mathrm{C}$ and inactivated for $5^{\prime}$ at $80^{\circ} \mathrm{C}$. Subsequently, the sample was separated by gel electrophoresis to excise the target fragment generated by restriction cleavage.

\subsubsection{Ligation of DNA}

Ligation is a technique to ligate two DNA fragments. It is directed by the enzyme DNA ligase requiring ATP, which then forms two covalent phosphodiester bonds between the 3' hydroxyl end and the 5' phosphate end.

Purified DNA fragment and the linearised plasmid were ligated. With respect to the fragment size, a certain amount of insert DNA (usually in the range of $20 \mathrm{ng}$ ) and $50 \mathrm{ng}$ plasmid DNA were mixed in a molar ratio of 3:1 with 5 U T4 DNA ligase and 1x ligase buffer (including ATP) in a final volume of $10 \mu \mathrm{l}$ at RT for 30'. T4 DNA ligase activity was inactivated by incubation at $65^{\circ} \mathrm{C}$ for $10^{\prime}$. Ligated plasmid was subsequently transformed into E.coli or stored at $-20^{\circ} \mathrm{C}$ for later transformation.

\subsection{Plasmid transformation of competent Escherichia coli (E.coli)}

Transformation of bacteria is based on the direct uptake of exogenous DNA through the membrane and is used for the amplification of plasmid DNA The plasmid usually encodes for a bacterial antibiotic resistance gene and enables selection of positively transformed clones (later referred to as E.coli clones). 
For plasmid transformation, chemically competent E.coli (see table 2.8) were removed from $-80^{\circ} \mathrm{C}$ and thawed on ice. For transformation of freshly ligated plasmid DNA, 3-5 $\mu \mathrm{l}$ of ligation mix (corresponds to 20-40 ng DNA) was added to $5 \times 10^{7} \mathrm{cfu} / \mu \mathrm{g}$ of pUC19. For re-transformation of purified plasmid DNA, $10 \mathrm{ng}$ was added to $5 \times 10^{7} \mathrm{cfu} / \mu \mathrm{g}$ of pUC19. The DNA/cell mix was incubated for 30' on ice. Subsequently, the mix was incubated at $42^{\circ} \mathrm{C}$ for $45^{\prime \prime}$ and then replaced on ice for 2'. One ml LB-agar was added, the transformed cells were agitated at $200 \mathrm{rpm}$ at $37^{\circ} \mathrm{C}$ for $30^{\prime}$. The cells were plated on two LB-Ampagar plates. Therefore, $100 \mu \mathrm{l}$ or $400 \mu \mathrm{l}$, of cell transformation mixture, respectively, were transferred to a solid nutrient agar-Amp plate and evenly dispersed using a Drigalski spatula. The plates were incubated at $37^{\circ} \mathrm{C}$ over night and subsequently stored at $4^{\circ} \mathrm{C}$. Dehydration of the plates was avoided by parafilm sealing.

\subsubsection{Plasmid isolation from bacterial clones}

Besides other methods, plasmid DNA can be isolated by adsorption to a silica membrane under certain salt conditions. Small-scale isolation of plasmid DNA was accomplished for further molecular analysis. For cell culture applications the endotoxin-free large scale plasmid isolation was performed.

\subsection{Small-scale plasmid isolation}

Positive clones were picked from the LB-Amp-agar plate with a sterile pipette tip and transferred to a $15 \mathrm{ml}$ tube including $3 \mathrm{ml}$ selective LB-medium including $100 \mu \mathrm{g} / \mathrm{ml}$ ampicillin. The bacterial culture was incubated at $250 \mathrm{rpm}$ for $14-16 \mathrm{~h}$ at $37^{\circ} \mathrm{C}$. On the following day $2 \mathrm{ml}$ of bacterial culture was transferred to a $2 \mathrm{ml}$ tube and pelleted at $6800 x$ for 3' at RT followed by an alkaline lysis of the cells (Birnboim, 1983) using a commercial kit (Qiagen plasmid mini kit) according to the manufacturer's instruction. The plasmid DNA was eluted in $50 \mu \mathrm{l}$ NF H2O and concentration was determined by spectrophotometry. The remaining culture was kept at $4^{\circ} \mathrm{C}$ for later applications (see next section) or long-term storage.

\subsection{Large-scale plasmid isolation}

In order to isolate large amounts of endotoxin-free plasmid DNA from positive clones $100 \mathrm{ml}$ selective LB-medium including $100 \mu \mathrm{g} / \mathrm{ml}$ ampicillin was inoculated with $100 \mu \mathrm{l}$ 
small scale culture (see above) and incubated at $250 \mathrm{rpm}$ for $14-16 \mathrm{~h}$ at $37^{\circ} \mathrm{C}$. Bacterial cells were harvested at $6000 \mathrm{xg}$ for $15^{\prime}$ at $4^{\circ} \mathrm{C}$. The pellet was resuspended in $10 \mathrm{ml}$ buffer 1 and further steps were performed according to the manufacturer's instructions (Qiagen EndoFree plasmid purification kit). As an exception to these instructions, precipitation and washing of DNA was accomplished at $6000 \mathrm{xg}$ for $1 \mathrm{~h}$ at $4^{\circ} \mathrm{C}$. The pellet was resuspended in $200 \mu \mathrm{l} N \mathrm{H}_{2} \mathrm{O}$ and concentration was determined using spectrophotometry.

\subsubsection{Long-term storage of E.coli strains}

For long-term storage $1 \mathrm{ml}$ bacterial culture was pelleted at 5000xg for 1', LB-medium was slightly discarded and the pellet was resuspended in $500 \mu \mathrm{l} 80 \%$ glycerol. The respective E.coli clone was immediately stored at $-80^{\circ} \mathrm{C}$. For recovery of a strain from $80^{\circ} \mathrm{C}$, a small part of the frozen solid was scraped off with a sterile pipette tip and transferred to $3 \mathrm{ml}$ selective LB medium.

\subsubsection{Sanger sequencing}

Sequencing was performed in order to determine correct primary sequence of the transgene. It is based on the chain determination method developed by Frederick Sanger (Sanger, Nicklen, and Coulson, 1977), modified to the dye terminator sequencing (Applied Biosystems). It uses extension of a template DNA by a DNA polymerase starting from a complementary oligonucleotide. The four desoxyribonucleotides are mixed with small amounts of differently fluorescently labelled di-desoxynucleotides, which terminate the replication reaction after being incorporated. During repeating cycles, terminator fragments accumulate which are separated by capillary electrophoresis and then detected.

Purified plasmids carrying PrP transgene were sequenced using the BigDye terminator sequencing kit. Therefore, $250 \mathrm{ng}$ plasmid was diluted in $0.75 \mathrm{x}$ sequencing buffer including $1 \mu \mathrm{l}$ big dye and $330 \mathrm{nM}$ sequencing primer to a final volume of $10 \mu \mathrm{l}$. The reaction conditions were the following: 25 repeating cycles of $30^{\prime \prime}$ at $96^{\circ} \mathrm{C}, 15^{\prime \prime}$ at $50^{\circ} \mathrm{C}$

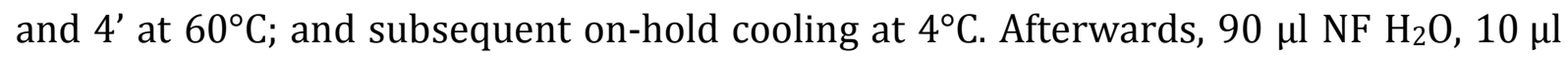
$3 \mathrm{M}$ sodium acetate (pH 5.5) and $250 \mu \mathrm{l} 96 \%$ ethanol was added to the sequencing 
reaction and precipitated at $16^{\prime} 000 \mathrm{xg}$ for $10^{\prime}$ at RT. The pellet was washed in $250 \mu \mathrm{l}$ $70 \%$ ethanol at $16^{\prime} 000 \mathrm{xg}$ for 5' at RT. Afterwards, the pellet was dried in a SpeedVac for $5^{\prime}$ at RT and sequenced by the ABI PRISM 3130xL Genetic Analyzer (Applied Biosystems).

\subsubsection{Protein biochemistry}

\subsubsection{Homogenization}

\subsection{Homogenization of brain tissue}

Brain homogenate was used for inoculation of cells. Therefore, 8 CWD infected whitetailed deer, were necropsied after showing characteristically CWD-associated clinical signs. The brain tissue of these animals was previously tested for PrPCWD by histopathology, IHC and Western blot (Hamir et al., 2008) and distributed to the DPZ for inoculation studies. $100 \mathrm{mg}$ amount of frozen brain tissue was cut in small pieces, weighted and transferred to a reaction tube. A $10 \% \mathrm{w} / \mathrm{v}$ homogenate was produced by dissolving the pieces in 1x DPBS, and homogenization with a sterile disposable pistil. The homogenates of 8 CWD-positive animals were pooled and stored at $-80^{\circ} \mathrm{C}$. Additionally, a pool of 3 non-infected animals was produced by the same procedure.

\subsection{Homogenization of cultured cells}

In order to infect transgenic cells with cell homogenate, cells were pelleted and cell density was determined. The pellet was resuspended in PBS and adjusted to $10^{7}$ cells/ml. Subsequently, the cell suspension was homogenized using a disposable pistil. The sample was subjected to 3 repetitive freeze-thaw cycles, each 30 '.

\subsubsection{Ultracentrifugation preparation}

In order to detect PrPres in infected cultures, cells were harvested, DNAse I digested and applied to ultracentrifugation. Therefore, cells were scraped from the well/dish in $1 \mathrm{ml}$ DPBS, an aliquot for cell density determination was counted and cells were pelleted at 2'000xg for 2' at RT. The pellet was resuspended in $1 \mathrm{ml} 1 \% \mathrm{~N}$-lauroylsarcosine 
(dissolved in PBS) and $10 \mu \mathrm{l}$ DNase I was added to a final concentration of $10 \mu \mathrm{g} / \mathrm{ml}$. The mix was incubated for $30^{\prime}$ at $37^{\circ} \mathrm{C}$. The DNase I addition was repeated and incubation was prolonged for another 30'. Afterwards, the sample was transferred to ultracentrifugation tubes, inserted into the ultracentifugation rotor TFT 80.4 with correct balancing and centrifuged at $100^{\prime} 000 \times \mathrm{xg}\left(39^{\prime} 000 \mathrm{rpm}\right)$ for $2.5 \mathrm{~h}$ at $4^{\circ} \mathrm{C}$. The supernatant was removed and the pellet was resuspended in $100 \mu \mathrm{l}$ DPBS. The sample was applied to PK digest (2.2.3.3) or stored at $-20^{\circ} \mathrm{C}$.

\subsubsection{Proteinase $\mathrm{K}$ digest}

Proteinase $\mathrm{K}(\mathrm{PK})$ is a serine endopeptidase belonging to the peptidase family S8 (Ebeling et al., 1974). Due to PK digestion under certain conditions endogenous PrPc (PK sensitive) and infectious PrPsc (PK-resistant) can be discriminated by immunoblotting (McKinley, Bolton, and Prusiner, 1983). Adding a proteinase inhibitor can terminate the hydrolysis.

Purified samples were thawed and again incubated with $5 \mu$ g DNAse I for $10^{\prime}$ at $37^{\circ} \mathrm{C}$ to dissolve the pellet. Afterwards, $50 \mu \mathrm{l}$ of sample (corresponds to $\sim 10^{6}$ cells) was incubated with $1 \mu \mathrm{g} / \mathrm{ml}$ or $10 \mu \mathrm{g} / \mathrm{ml} \mathrm{PK}$, respectively, and $5 \mu \mathrm{l} 1 \% \mathrm{~N}$-laurylsarcosine in a total volume of $56 \mu \mathrm{l}$. The PK digest was performed for $1 \mathrm{~h}$ at $37^{\circ} \mathrm{C}$ with gentle agitation. The reaction was terminated by adding $4 \mu \mathrm{l} 1 \%$ pefabloc $\left(1 \%\right.$ in $\left.\mathrm{H}_{2} \mathrm{O}\right)$. Samples were directly diluted in 4x PAGE LDS-loading buffer to a final volume of $80 \mu \mathrm{l}$ to $1 \mathrm{x}$ and stored at $-20^{\circ} \mathrm{C}$ prior PAGE.

\subsubsection{Preparation of lysate from non-infected cells}

A cryo vial with transgenic cells $\left(5 \times 10^{6}\right.$ cells $)$ was thawed and pelleted. The pellet was resuspended in $1 \mathrm{ml}$ lysis buffer containing $10 \mathrm{mM}$ TRIS HCl (pH 7.4), $150 \mathrm{mM} \mathrm{NaCl}, 1 \%$ (v/v) Triton-X $100(\mathrm{w} / \mathrm{v}), 0.1 \%$ DOC and $1 \%$ SDS. The lysate was passed through a QIAshredder column and the flow-through was incubated for $15^{\prime}$ at $95^{\circ} \mathrm{C}$ with intense shaking. The lysates were diluted in 4x PAGE LDS-loading buffer, $10^{\prime}$ incubated at $96^{\circ} \mathrm{C}$ and directly applied to Western blot analysis or stored at $-20^{\circ} \mathrm{C}$. 


\subsubsection{Western blot}

Western blotting is used as a method to detect specific proteins in a sample by antibodies. It is based on gel electrophoretic separation (PAGE), the transfer of proteins to a membrane with subsequent blocking to prevent non-specific binding of the antibodies. The following immunostaining with specific antibodies and chemiluminescent detection is accomplished to detect the protein of interest (Burnette, 1981; Renart, Reiser, and Stark, 1979).

\subsection{Polyacrylamide gel electrophoresis for separation of proteins}

Polyacrylamide gel electrophoresis (PAGE) is a widely used technique in molecular biology and biochemistry for separation of proteins (or nucleic acids) according to their electrophoretic properties (Davis BJ, 1959; Raymond and Weintraub, 1959). Here, PAGE was used for the separation of lysates from cells.

Therefore, samples which were diluted in PAGE-LDS loading buffer were boiled for 10' at $95^{\circ} \mathrm{C}$ and then cooled on ice. Precast $12 \%$ Bis-Tris $1 \mathrm{~mm}$ gels fixed into the PAGE device according to the manufacturer's instructions. The inner and the outer chamber of the device were filled with MOPS-SDS buffer. The samples were load into the slots of the gel and a protein marker was used for mass weight determination. The electrophoretic separation per gel was performed at $70 \mathrm{~mA}$ for $90^{\prime}$.

\subsection{Electroblotting}

Proteins are transferred to a membrane after PAGE. It is based on current flow in combination with a protein transfer buffer to mobilise proteins from the gel to the membrane.

Therefore, PVDF membrane was cut into the size of the PAGE gel. PVDF membrane was activated by incubation in $100 \%$ methanol for 10", short washing in DI H2O and 10' incubation in transfer buffer at RT. The gel (2.2.3.5.1) was removed from the PAGE device and taken off from the plastic cassette using a gel knife. The gel and the membrane were layered in filter papers and sponges and transferred to the blotting device according to the manufacturer's instructions. The inner blotting chamber was

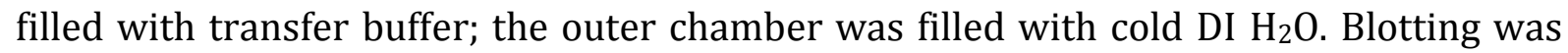
performed at $135 \mathrm{~mA}$ for $70^{\prime}$. After blotting, the membrane was rinsed with TBS and 
then incubated in $4 \mathrm{M} \mathrm{GdnSCN}$ for 30' with gentle agitation and then 3 times for 5 " and 3 times for 10' in $30 \mathrm{ml}$ TBS-Tween at RT with gentle agitation.

\subsection{Immunostaining}

Detection of specific proteins was accomplished by immunostaining. It is based on the recognition and binding of a specific antibody to a specific target protein and the following detection. First, the membrane is placed in a protein solution and the membrane is saturated at all places, where no protein is attached. Then, a target protein specific primary $\mathrm{Ab}$ is incubated on the membrane. Consecutively incubation with the secondary antibody recognised the primary antibody. It is coupled to an enzyme, which converts a metabolic substrate leading to the chemiluminescent emission of light. The emission can then expose a light sensitive X-ray film or can be detected by a CCD camera connected to an imager device.

The membrane was incubated in 1x TBS-T (for later detection using mAB ICSM-18) or $0.2 \%$ Casein (all other primary antibodies) for 60' at RT. After the blocking step, membranes were incubated with primary antibody diluted in TBS-T (for detection using mAB ICSM-18) or $0.02 \%$ Casein-TBS (all other primary antibody) in a total volume of $15 \mathrm{ml}$ over night at $4^{\circ} \mathrm{C}$. The following day, the membrane was washed 3 times 5", 3 times in TBS-T for 5', one time for 10' and three times 20' and afterwards incubated with secondary antibody diluted in TBS-T (if prior use of ICSM-18) or $0.02 \%$ Casein-TBS (remaining antibodies) in a total volume of $15 \mathrm{ml}$ for $1 \mathrm{~h}$ at RT. The secondary antibody (A3688 for primary mouse antibodies, A9919 for primary rabbit antibody) was washed out as described for the primary antibody. All steps were performed on a shaker with gentle agitation (at 40-60 rpm). Subsequently, the membrane was dried in paper towels and put inside a development folder. Following the manufacturer's instructions step-bystep, 20 drops of CDP-Star substrate were used to cover the membrane and visualise the target proteins. After 30' incubation light emission was recorded by exposure to a luminescent imager or X-ray films. If membrane was reprobed with another primary antibody, membrane was incubated in TBS-T for $1 \mathrm{~d}$ at $4^{\circ} \mathrm{C}$ with gently agitation. Afterwards membrane was incubated in stripping buffer for 10' at RT and exchanged with fresh buffer for additional 10' with agitation. The stripping buffer was washed out twice in PBS for 10' and consecutive twice in TBST for 5'. After this washing procedure, membrane was ready for initial blocking step. 


\subsubsection{Flow cytometry}

Flow cytometry is a technique to examine single cells in a continuous flow by passing them alongside light beams (usually laser light). One laser light is focused in line with the cell stream (forward scatter, FSC), which detects the cell volume; and a second is positioned perpendicular to the stream (side scatter, SSC) detecting the inner complexity of the cell. Due to excitation of a fluorophore, light is emitted and a fluorescent detection device collects the information.

Here, flow cytometry was used for the detection of PrP on the cell surface of trangenic cell lines. Therefore, cells were rinsed with DPBS, detached by scraping and cell density was determined. One million cells were pelleted at 100xg for 2' at RT, resuspended in $50 \mu \mathrm{l}$ DPBS and incubated with titrated amounts of primary antibody for 30' at RT. Residual antibodies were washed off by the addition of $1 \mathrm{ml}$ DPBS and subsequent centrifugation. The pellet was again resuspended in $50 \mu \mathrm{l}$ DPBS and incubated with $5.5 \mu \mathrm{g}$ of secondary antibody for $30^{\prime}$ at RT, which is coupled to the fluorophore Rphycoerythrin-Cy5 (see table 2.9,). Residual secondary antibody was washed off and cells were fixed in $150 \mu$ freshly prepared $0.37 \%$ paraformaldehyde. The suspension was transferred to flow cytometry tubes and stored at $4^{\circ} \mathrm{C}$ prior use. Subsequently, cell suspensions were measured in the flow cytometer LSRII. The data were processed by the software DIVA and FlowJo 5.0.

\subsubsection{Computational biology}

\subsubsection{Sequence analysis}

In order to compute nucleotide sequences for primer design the software Vector NTI advanced 11.0 was used. Directly sequenced parts of plasmid DNA were processed and further analyzed by ContigExpress and AlignX (included in the Vector NTI installation package). Homology of protein sequences was analyzed using Vector NTI and AlignX. Data base sequences were retrieved from the server of the National Center for Biotechnology Information (NCBI). 
Table 2.14: Data analyses and software

\begin{tabular}{ll}
\hline application & name \\
\hline microscopic images & CellF (Olympus) \\
flow cytometry & DIVA 6.0 (BD bioscience), FlowJo 8.7.1 (Tree Star Inc.) \\
reference data base & Endnote X (Thomson Reuters) \\
gel documentation & INTAS application software (INTAS) \\
membrane documentation & INTAS application software (INTAS) \\
graphical processing & GraphPad Prism 5.01 (GraphPad software, Inc.) \\
QPCR & SDS 7500 system software (Applied Biosystems) \\
data processing & Microsoft Office Excel, Word, Power Point 2003 \\
& (Microsoft Cooperation) \\
\hline
\end{tabular}

\subsubsection{Safety measures}

The production of transgenic cell lines and the infection of the cells was authorized by the trading standards office in Göttingen with the permit number Az. 40611/0501/655. Infection of human transgenic cells with prion-infectious agents and Western blot analysis was accomplished in a bio safety level 3 laboratory (BSL-3). For this purpose, the lab staff was dressed in water resistant lab coats and wore two pairs of medical gloves and protection goggles. During production of homogenates from infected brain tissue, a protective mask was worn. Potentially contaminated laboratory equipment was decontaminated by incubation in a $1 \mathrm{M} \mathrm{NaOH}$ bath for at least 60'. After immunoblotting the PVDF membrane was inactivated by incubation in $4 \mathrm{M} \mathrm{GdnSCN}$ for 60 ' and then was further processed under BSL-2 conditions. Laboratory waste was inactivated at $134^{\circ} \mathrm{C}$ for 60'. Molecular biology methods were accomplished under BSL-2 conditions.

\subsubsection{Animal Experiments}

Cynomolgus macaques (Macaca fascicularis) were maintained in social groups of three monkeys and housed in a microbiological containment level BSB3 facility. Ethics approval for the study was issued by the Lower Saxony Ministry for consumer protection and food safety (Az. 33.9.42502-04/070/07). Animal experimentation was performed in accordance with section 8 of the German Animal Protection Law in 
compliance with EC Directive 86/609. Primate experiments were carried out at the German Primate Centre. To evaluate interspecies transmission of CWD to non-human primates, 22 monkeys were inoculated with CWD tissue or mock tissue, respectively, via different inoculation routes. Brain and muscle tissue was analysed for their infectivity in the well-established TSE cynomolgus macaque model modelling the human risk in CWD transmissibility. The brain inoculum was prepared as described in section.2.2.3.1.1. The muscle tissue was weighted, cut into pieces and $100 \%$ (w/v) PBS was added. Next, the tissue was homogenized by a hand mixer, aliquoted á $80 \mathrm{ml}$ per syringe and stored at $80^{\circ} \mathrm{C}$ prior use. $21 \mathrm{M}$. fascicularis were inoculated by CWDWTD agent via oral, intracerebral, blood transfusion route and by scarification. Two animals were orally inoculated by a stomach tube with $2 \mathrm{~g}$ of brain homogenate $(40 \mathrm{ml}$ of $5 \%(\mathrm{w} / \mathrm{v})$ homogenate) for 5 weeks and 1 animal with $5 \times 2$ g mock brain homogenate, respectively. 3 animals were orally inoculated with $80 \mathrm{ml}$ muscle homogenate twice a week, which corresponds to $80 \mathrm{~g}$ muscle tissue per week. Two animals were intracerebrally inoculated with $10 \mathrm{mg}$ CWD WTD brain homogenate $(100 \mu \mathrm{l}$ of $10 \%$ (w/v) homogenate) and 1 animal with $10 \mathrm{mg}$ mock brain homogenate by the use of a Hamilton needle. Additionally, 3 animals were inoculated via surgical implantation of a CWDWTD brain homogenate contaminated steel wire and in 1 animal a mock steel wire. 2 animals were inoculated by implantation of a CWD ${ }^{E L K}$ brain homogenate contaminated steel wire with 1 corresponding mock animal. For the intracerebral inoculation experiments, the cranium was fixated in a stereotactic device. Drs. Christiane Stahl-Hennig and Walter Schulz-Schaeffer, together with Dirk Prüße (anaesthesia) accomplished the surgical procedure. Furthermore, in 2 animals a dermal scarification was accomplished by microdermal lesions and subsequent surface application of CWDWTD brain homogenate. Additionally, 3 animals were intravenously inoculated with up to $15 \mathrm{ml}$ plasma of a preclinical CWD inoculated cynomolgus macaque (kindly provided by B. Race, National Institute of Allergy and Infectious Diseases, NIH, Hamilton, MT, USA). The blood transfusion experiment is carried out at the French Atomic Energy Commission (CEA), Fontenay-aux-Roses, France by Drs. J.P. Deslys and E. Comoy. Table 2.15 summarises the CWD animal inoculation study. If not stated otherwise, the inoculation procedure was developed, modified and finally accomplished in close cooperation with the veterinary team and the animal keepers of the German Primate Center. 
Table 2.15: CWD inoculation study in M. fascicularis

\begin{tabular}{|c|c|c|c|c|}
\hline $\begin{array}{l}\text { inoculation } \\
\text { route }\end{array}$ & inoculum & amount & \# animals & $\begin{array}{l}\text { start } \\
\text { inoculation }\end{array}$ \\
\hline oral & muscle CWDWTD & $80 \mathrm{~g} /$ week & 3 & September 2009 \\
\hline oral & brain CWDWTD & $\begin{array}{l}2 \mathrm{~g} / \text { week for } 5 \\
\text { weeks }\end{array}$ & 2 & April 2009 \\
\hline oral & brain mock & $\begin{array}{l}2 \mathrm{~g} / \text { week for } 5 \\
\text { weeks }\end{array}$ & 1 & April 2009 \\
\hline intracerebral & brain CWDWTD & $10 \mathrm{mg}$ & 2 & July 2009 \\
\hline intracerebral & brain mock & $10 \mathrm{mg}$ & 1 & July 2009 \\
\hline $\begin{array}{l}\text { intracerebral } \\
\text { steel wire }\end{array}$ & $\begin{array}{l}\text { brain CWDWTD } \\
\text { contaminated } \\
\text { steel wire }\end{array}$ & - & 3 & October 2009 \\
\hline $\begin{array}{l}\text { intracerebral } \\
\text { steel wire }\end{array}$ & WTD mock brain & - & 1 & October 2009 \\
\hline $\begin{array}{l}\text { intracerebral } \\
\text { steel wire }\end{array}$ & $\begin{array}{l}\text { brain } \text { CWD }^{\text {ELK }} \\
\text { contaminated } \\
\text { steel wire }\end{array}$ & - & 3 & November 2010 \\
\hline $\begin{array}{l}\text { intracerebral } \\
\text { steel wire }\end{array}$ & ELK mock brain & - & 1 & November 2010 \\
\hline $\begin{array}{l}\text { dermal } \\
\text { scarification }\end{array}$ & brain CWDWTD & - & 2 & May 2009 \\
\hline intravenous & $\begin{array}{l}\text { preclinical } \\
\text { plasma }\end{array}$ & $15 \mathrm{ml}$ & 3 & May 2009 \\
\hline
\end{tabular}

Bimonthly, $1 \mathrm{ml}$ cerebrospinal fluid (CSF) and $15 \mathrm{ml}$ citrate blood was collected from each animal. Blood was fractioned by ficoll gradient separation and the fractions were stored at $-80^{\circ} \mathrm{C}$. 


\section{Results}

The aim of this thesis was the establishment of a cell culture based system to estimate whether a prion-infectious agent from one species can lead to the infection of another species. Due to the zoonotic character of prion diseases latent prion reservoirs pose a latent risk to consumers of animal products. In particular, bovine spongiform encephalopathy (BSE) and chronic wasting disease (CWD) in captive animal herds remain an issue. To address this question we aimed to generate transgenic cell lines that stably overexpress the prion protein derived from different species. With subsequent inoculation of the cell lines with different prion-infectious agents, we intended to mimic the respective species barrier. To estimate the transmissibility of CWD, cell lines overexpressing PrP from different species were tested for their susceptibility. First, respective genes from $H$. sapiens as a potential recipient, next from $M$. fascicularis and $S$. sciureus as non-human primate model species and last from O. virginianus as CWD susceptible species were chosen.

The squirrel monkey (S. sciureus) has been used as non-human primate model in prion research and it was possible to transfer sheep Scrapie and CWD to this new world monkey (Gibbs et al., 1980; Marsh et al., 2005; Race et al., 2009). The white-tailed deer (O. virginianus) is affected by CWD and was therefore chosen as a positive control. It was also shown, that the cynomolgus macaque (M. fascicularis) is a suitable non-human primate model. This old world monkey species have been used to estimate the zoonotic potential of BSE and was the first proof that BSE was causative for human vCJD (Lasmezas et al., 2005). The use of non-human primate models is highly elaborate but necessary due to its close relation humans. Nevertheless, due to the long preclinical incubation time of in vivo experiments, prion research is very time-intense. Therefore, cell culture-based inoculation experiments can be an elegant and shorter way to predict the transmissibility of prion diseases such as CWD. 


\subsection{Generation of retroviral expression plasmids encoding the prion protein of different species}

\subsubsection{Cloning of PrP into pJET1.2 vector}

In order to generate stable transgenic PrP cell lines using retroviral transduction, first PrP had to be inserted into a retroviral expression vector. This gene delivery system leads to the efficient and stable integration of the transgene into the genome of a target cell. Therefore, total RNA was isolated from whole blood from M. fascicularis and $H$. sapiens and from brain tissue derived from S. sciureus and O. virginianus, respectively, (2.2.2.1). When the RNA concentration was below $10 \mathrm{ng} / \mu \mathrm{l}$ or the salt concentration was too high, ethanol/sodium precipitation was performed (2.2.2.4). Subsequently, the RNA was used for cDNA synthesis (2.2.2.5). Amplification of PrP ORF was performed by PCR using a proof reading DNA polymerase (2.2.2.6) and the corresponding primer pairs for each species (table 3.1).

\section{Table 3.1: Primer pairs for the amplification of PrP}

\begin{tabular}{lll}
\hline species & primer pair & amplicon size \\
\hline M. fascicularis, S. sciureus & Mafa PrP Fwd, Mafa PrP Rev & 905,925 \\
H. sapiens & Mafa PrP Fwd, Homo PrP Rev & 907 \\
O. virginianus & WTD PrP Fwd, WTD PrP Rev & 872 \\
\hline
\end{tabular}

The primer pairs were designed to bind the 5'-upstream and 3'-downstream region of the PrP transcript and enabled full-length translation from start to stop codon without the interference of species-specific transcriptional regulation signals. The amplified fragments were separated by agarose gel electrophoresis (2.2.2.7), excised and purified (2.2.2.8). Next, the PCR fragments were ligated into the pJET1.2 plasmid (2.2.2.10) and the newly generated plasmids were then transferred into E.coli via transformation (2.2.2.11). These cells were grown on agar plates with selection for ampicillin resistance, which is encoded by the plasmid. Grown E. coli clones were picked and expanded. Next, plasmid DNA was isolated in small scale (2.2.2.12). The cloned sequence was checked for accuracy by Sanger sequencing (2.2.2.14). The sequencing primers (table 3.2) were designed to bind upstream and downstream of the MCS to enable full-length sequencing 
of the PrP inserts including start and stop codon. Furthermore, two internal primers were designed to ensure whole sequence coverage.

Table 3.2: Primers used for PrP sequencings

vector construct

sequencing primer

pJET1.2-(homo)PrP, pJET1.2-(mafa)PrP, pJET1.2 Fwd, pJET1.2 Rev, Mafa PrP int

pJET1.2-(sai)PrP, pJET1.2-(wtd)PrP Fwd, Mafa PrP int rev

pBABE-(homo)PrP, pBABE-(mafa)PrP, pB MCS-40, pB MCS+200, Mafa PrP int pBABE-(sai)PrP, pBABE-(wtd)PrP Fwd, Mafa PrP int rev

The sequencing results were aligned to their respective reference sequences from public databases and the accuracy of the sequence was assessed. All chosen clones, carrying a plasmid coding for PrP from the species H. sapiens (reference sequence: NM183079), $M$. fascicularis (reference sequence: AB125193), and O. virginianus (reference sequence: AY360089) were identical to their reference sequence. In contrast, the PrP sequence from S. sciureus was not identical to any of the four available NCBI database entries (U15165, FJ796964, FJ796962 and U08310). The corresponding nucleotide sequence (7.1) was in silico translated into an amino acid sequence (7.2) and aligned to the four different database sequences. As shown in figure 3.1 the newly identified PrP sequence has an insertion of $8 \mathrm{AA}$ within the octarepeat region of $\operatorname{PrP}$ and three sequence variations in comparison to other database sequences. This new PrP genotype of a squirrel monkey was submitted to the NCBI (National Centre for Biotechnology Information) database (submission ID: 1485013).

$\begin{array}{lllllll} & (1) & (10) & {[]} & (90) & {[]} & (170) \\ \text { DPZ } & \text { MANLGYWML } & \text { VLFVATWSDL } & {[]} & \text { QPHGGGWGQG } & {[]} & \text { YKPPVDQYSNQ } \\ \text { U15165 } & \text { MANLGYWML } & \text { VLFVATWSDL } & {[]} & -------Q G & {[]} & \text { YKPPVDQYSNQ } \\ \text { U08310 } & \text { MANLGCWML } & \text { VLFVATWSDL } & {[]} & \text { QPHGGGWGQG } & {[]} & \text { YRPPVDQYSNQ } \\ \text { FJ796964 } & \text { MANLGCWML } & \text { VLFVATWSDV } & {[]} & \text { QPHGGGWGQG } & {[]} & \text { YKPPVDQYSNQ } \\ \text { FJ796962 } & \text { MANLGCWML } & \text { VLFVATWSDL } & {[]} & \text { QPHGGGWGQG } & {[]} & \text { YKPPVDQYSNQ }\end{array}$

Figure 3.1 Amino acid alignment of squirrel monkey (S. sciureus) PrP

Parts of the protein sequence of all four available squirrel monkey PrP database entries (U15165, U08310, FJ796964 and FJ796962) were aligned to the DPZ PrP sequence cloned from a squirrel monkey from the Max-Planck Institute Munich. (\#) indicate the position of amino acid; square brackets indicate excluded sequence parts. Letters in bold indicate variations among the sequences. 


\subsubsection{Cloning of PrP cDNAs into retroviral expression vector (pBABE)}

In order to generate stable transgenic cell lines expressing $\mathrm{PrP}$, the respective PrP ORF was inserted into the retroviral expression vector by using sticky-end cloning (2.2.2.9). PrP was amplified by PCR (2.2.2.6) using JET1.2-PrP of the respective species as template and the corresponding cloning primer pairs (table 3.3). These cloning primers include a cleavage site for a restriction enzyme identical to a corresponding cleavage site in the target vector and absent from the DNA insert.

\section{Table 3.3: Cloning primers for the amplification of PrP}

\begin{tabular}{lll}
\hline species & primer pairs & restriction enzymes \\
\hline H. sapiens, M. fascicularis, & Mafa PrP BamH1 fwd, BamH1, EcoR1 \\
S. sciureus & Mafa PrP EcoR1 rev \\
O. virginianus & WTD PrP EcoR1 fwd, EcoR1, Sal1 \\
& WTD PrP Sal1 rev & \\
\hline
\end{tabular}

The PCR products were separated by agarose gel electrophoresis (2.2.2.7), excised from the gel and purified (2.2.2.8). The PrP DNA fragment and the target vector pBABE were then applied to repeated restriction cleavage (2.2.2.9) leaving dsDNA pieces with ss protruding ends. The cleaved PrP fragments and linearised vectors were again separated by agarose gel electrophoresis. After purification of the fragments, both DNA ends were ligated (2.2.2.10) and subsequently transformed into E.coli (2.2.211). The transformed bacterial clones were grown on antibiotic selective agar plates, and clones were picked and expanded. Plasmid DNA was isolated (2.2.2.12.1) and sequencing with $3 x$ coverage (2.2.2.14) was accomplished to check correct insertion. In addition the DNA sequence was reassessed for mutations in the ORF. As described for subcloning of PrP into pJet1.2, all sequences were aligned to the corresponding reference sequence. In total, 64 sequencing reactions for 20 clones for 4 species ( 5 clones of each species) were performed. For each species, one mutation-free clone was chosen that was identical to the full-length PrP reference sequence of the respective species. For S. sciureus we took the clone with the highest consistency within the sequence alignment. In order to transfect the retroviral plasmid into a eukaryotic target cell lines which later induces the production of retroviral particles, high amounts of plasmid DNA from the tested clones 
were produced using an endotoxin-free plasmid isolation kit (2.2.2.12.2). Endotoxins are cell membrane components from bacteria, which can copurify during the plasmid isolation procedure. The endotoxin-free isolation exerts increased removal of endotoxins, which provides consistent transfection rates and comparable quantity and quality of the generated retroviral virus particles for subsequent transductions.

\subsection{Generation of cell lines overexpressing the prion protein of different species}

\subsubsection{Choice of cell lines suitable to examine the zoonotic potential of prion-infectious agents}

To analyse the species barrier of a prion disease it is necessary to minimise interference by the expression of endogenous prion protein. Thus, the murine hippocampus cell line HpL3-4, derived from the hippocampus region of a $\mathrm{PrP}^{0 / 0}$ mouse (Kuwahara et al., 1999) was chosen (kindly provided from Prof. T. Onodera, Tokyo). This cell line was previously shown to be permissive for infection with prion agents (Maas et al., 2007). The absence of the prion gene and thus the prion protein was validated by qPCR and immunoreactivity as described later.

Furthermore, the human neuroblastoma cell line SH-SY5Y was chosen for inoculation studies. Due to its neuronal origin this cell line should closely mimic the transmissibility of prion diseases to humans. Additionally, this cell line was already demonstrated to be susceptible for sCJD (Ladogana et al., 1995).

\subsubsection{Generation and transduction of replication defective retroviral particles carrying PrP of different species}

In order to establish stable transgenic cell lines, retroviral particles had to be generated. A highly efficient transfection protocol is necessary to ensure the uniformity and comparability among particle production to transduce the target cells. Technical optimisation should enhance the comparability of the subsequent experiments.

For high titre production of retroviral particles, HEK293FT cells were co-transfected (2.2.1.7) with three plasmids (Morgenstern and Land, 1990). The packaging plasmids pVPack-GP and pVPack-VSV-G encode for viral structure proteins. 


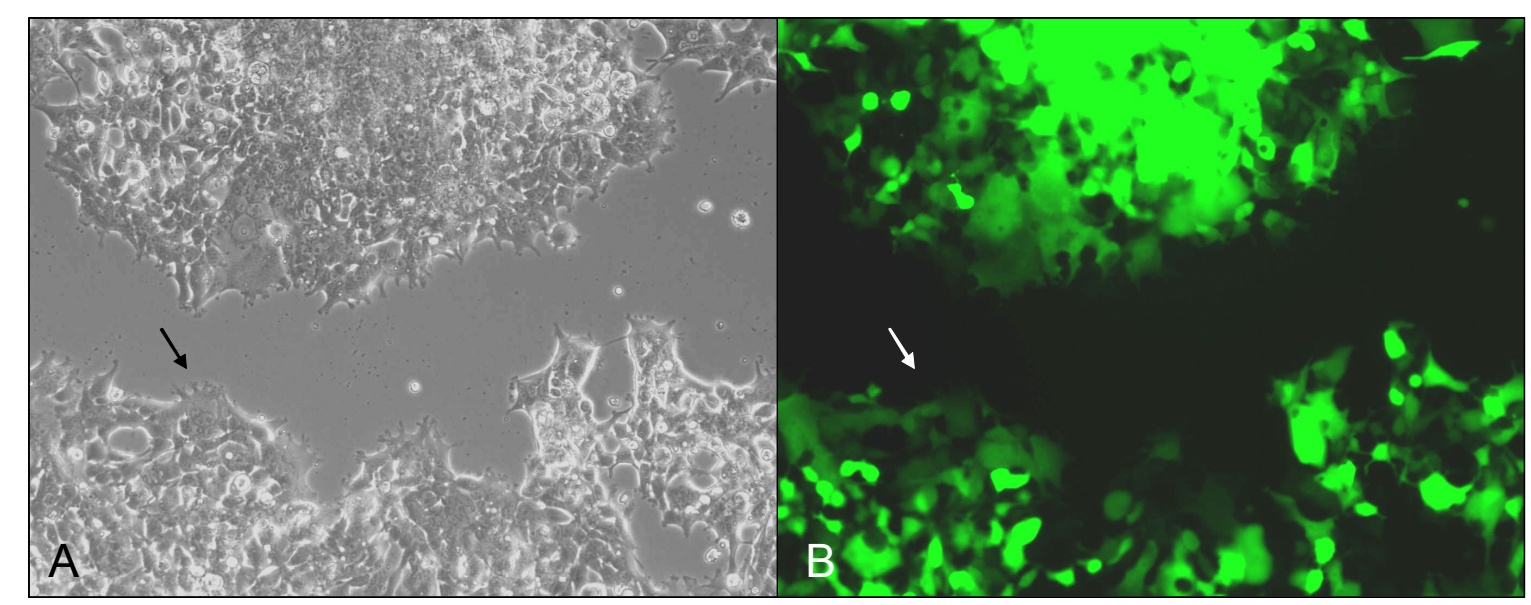

Figure 3.2 EGFP expressing HEK293FT after transfection with pBABE-EGFP

HEK293FT human embryonic kidney cells were transfected with pBABE-EGFP and analysed after $2 \mathrm{~d}$ for EGFP expression. Cells were visualised by phase contrast (A) and fluorescence (B) microscopy at $400 \mathrm{x}$ magnification. The arrows denote equivalent areas of the images.

The third retroviral plasmid (pBABE) carries the transgene that encodes for the prion protein from the species $H$. sapiens, $M$. fascicularis, $S$. sciureus or $O$. virginianus.

Successful transfection of pVPack-GP, pVPack-VSV-G and pBABE leads to the production of replication-defective viral particles that carry the prion transgene from these four different species. In addition to pBABE-PrP, HEK293 FT cells were co-transfected with the empty pBABE vector (later referred to as empty vector control $(E M C)$ ) or with pBABE-EGFP carrying the full-length sequence for the enhanced green fluorescent protein (EGFP). EMC was used to generate a more suitable internal negative control for qPCR, protein analysis and infection experiments than the conventional non-transduced wild type cells. They undergo the same experimental procedures such as retroviral transduction and antibiotic selection. The EGFP expression vector was used as both, a visual reporter and a transfection and transduction efficiency control. Due to the transfection with this vector, HEK293 FT cells express EGFP (figure 3.2), which was visualized by fluorescence microscopy.

Once the viral RNA carrying the PrP transgene is encapsulated together with gag, pol and $e n v$, the viral particles bud from the packaging HEK293FT cells and are released into the cell culture medium. The viral particles were collected and separated from cell debris. The combination of viral packaging vectors and retroviral expression vector coding for PrP generates retroviral particles, which are capable to transduce cells with transgenic PrP. As viral envelope (env) protein the VSV-G protein from the vesicular 
stomatitis virus was used. The VSV-G recognises a phospholipid (Yee, Friedmann, and Burns, 1994) that is present on all eukaryotic cells. It should mediate the transduction of either murine HpL3-4 or human SH-SY5Y cells. The viral particles bind to the target cell surface and release the viral RNA genomes carrying the full-length PrP ORF of the species H. sapiens, M. fascicularis, S. sciureus or O. virginianus. The PrP is flanked by LTRs, which are necessary for the integration into the host cell genome. The integrase protein, encoded in the pol gene of the pVPack-GP, mediates this process. Due to the separate arrangement of cis and trans-acting genes in the used transduction system, the produced retroviral particles are deficient in replication and only one round of infection can be completed. Thus transduced cells cannot propagate intact virus avoiding a safety concern while working with permanently prion infected cells.

\subsubsection{Estimating the transduction efficiency of replication-defective retroviral particles carrying EGFP transcript}

The efficiency of retroviral transduction was determined by transduction of cells with retroviral particles carrying the EGFP transgene. For this purpose packaging cells were transfected (2.2.1.7.1) with the packaging vectors pVPack-GP and pVPack-VSV-G and the retroviral expression plasmid pBABE-EGFP. The generated particles were harvested and purified (2.2.1.7.2). Two different transduction protocols were compared to estimate the maximum transduction efficiency. The efficiency of each protocol was monitored by EGFP expression in the HpL3-4 cells using fluorescence microscopy. In the first set-up, cells were transduced by covering the surface of the adherent cells with viral supernatant including DEAE-dextran used to facilitate binding of nucleic acids to the cell membrane and the entry via endocytosis (Gregory et al., 2003). Alternatively, cells were transduced by spinduction in combination with the addition of $3 \mu \mathrm{g} / \mathrm{ml}$ polybrene (Aubin et al., 1997; Rousculp, Goldsmith, and Garver, 1992). The EGFP expression was measured $4 \mathrm{~d}$ after transduction when cells show the highest expression of EGFP. DEAEdextran transduced cells show approximately $20 \%$ EGFP positive cells $4 \mathrm{~d}$ post transduction (figure 3.3-B). In comparison, polybrene-assisted spinduction increased the transduction efficiency to up $80 \%$ (figure 3.3-D). Thus, spinduction with polybrene was used to generate PrP transgenic cell lines. 


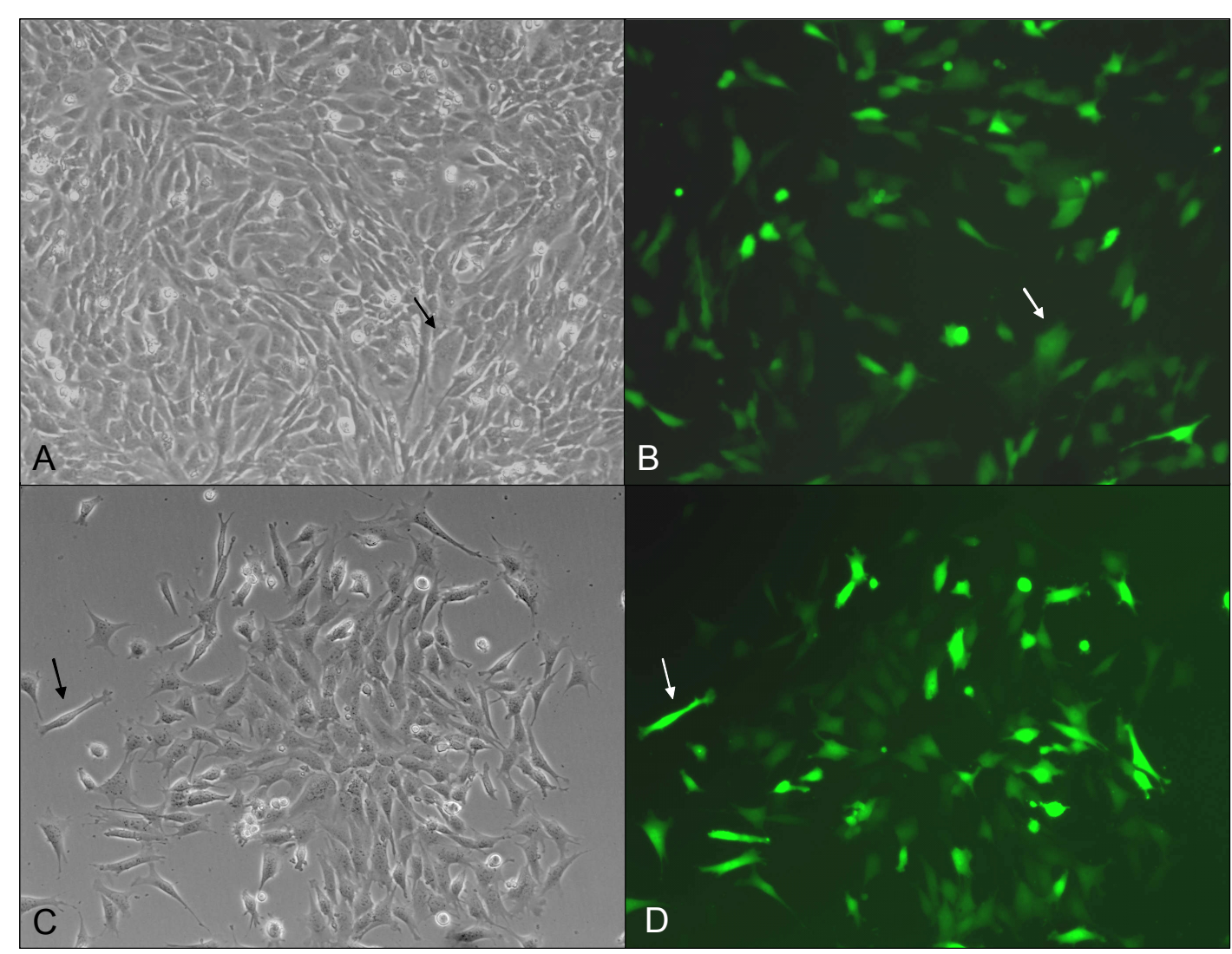

Figure 3.3 Transduction efficiency of viral particles carrying EGFP transgene in HpL3-4 cells

HpL3-4 cells were either transduced with retroviral particles carrying the EGFP transgene with DEAE-dextran (A, B) or polybrene with subsequent spinduction (C, D). A, C: Representative phase contrast images of HpL3-4 cells 4 days post transduction. B, D: corresponding fluorescence image of A, C. Cells were photographed at 400x magnification.

\subsubsection{Acute toxicity test of puromycin in HpL3-4 cells and SH-SY5Y}

The retroviral expression vector coding for PrP exhibits a selection marker that conveys resistance against the eukaryotic antibiotic puromycin. To select positively transduced cells, cells were treated with puromycin 4 days post transduction. To identify the minimal toxic dose for each cell line not inducing metabolic side effects, a suitable puromycin concentration had to be evaluated. But this dose had to delete nontransduced cells. HpL3-4 cells and SH-SY5Y were treated with increasing concentrations of puromycin. The maximal and minimal concentrations were chosen according to those used in HeLa (Ukekawa et al., 2007) and HEK293 cells (Pick et al., 2007). After addition of puromycin to HpL3-4 and SH-SY5Y cells were passaged for $4 \mathrm{~d}$ in culture medium. 


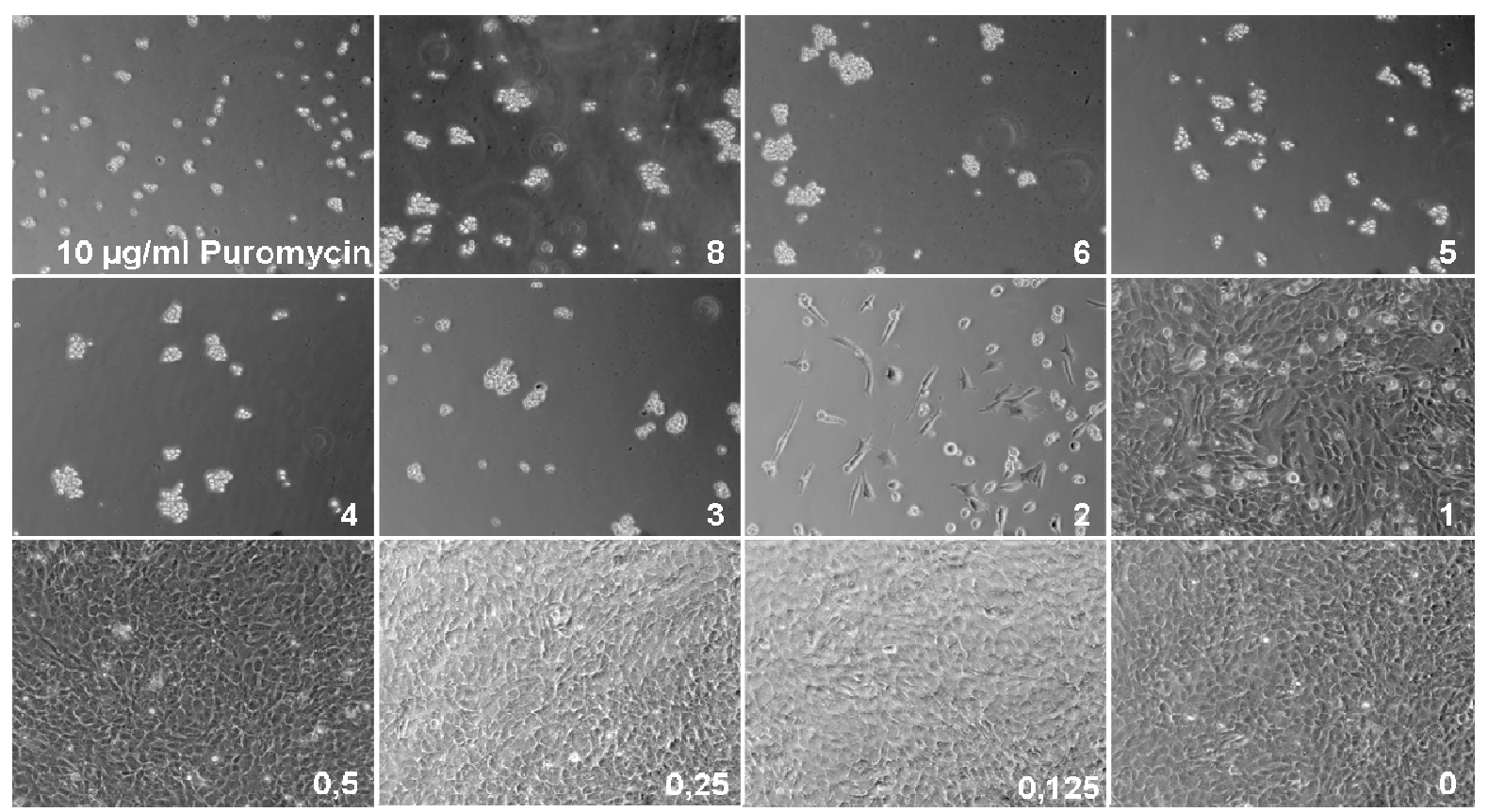

Figure 3.4 Toxicity of puromycin in HpL3-4 cells

HpL3-4 hippocampus neuronal cells were treated with decreasing concentrations of puromycin (10-0.125 $\mu \mathrm{g} / \mathrm{ml})$ or left untreated $(0 \mu \mathrm{g} / \mathrm{ml})$ for $4 \mathrm{~d}$ and imaged by phase contrast microscopy. Final concentrations of puromycin in $\mu \mathrm{g} / \mathrm{ml}$ were stated in white numbers in the lower right in each picture. All pictures were photographed at $400 \mathrm{x}$ magnification.

For this time cells did not survive in the highest puromycin concentration used $(10 \mu \mathrm{g})$ and cells were completely vital in the untreated negative control (figure 3.4). HpL3-4 cells showed no inhibition in growth using puromycin concentrations below $1 \mu \mathrm{g} / \mathrm{ml}$. Addition of $1 \mu \mathrm{g} / \mathrm{ml}$ puromycin decreased growth with a low percentage of detached cell bodies. $2 \mu \mathrm{g} / \mathrm{ml}$ puromycin induced strong inhibition of growth and the cells became shaped irregularly. In addition, a large percentage of cells were detached. At a concentration of $3 \mu \mathrm{g} / \mathrm{ml}$ puromycin all cells were detached and tend to form clusters..

Thus we have chosen $3 \mu \mathrm{g} / \mathrm{ml}$ puromycin for the following retroviral transduction of HpL3-4 cells as the minimal dose completely inhibiting cell growth and survival. Using a higher concentration of puromycin may also cause toxic side effects in resistant cells that may interfere with prion protein expression 


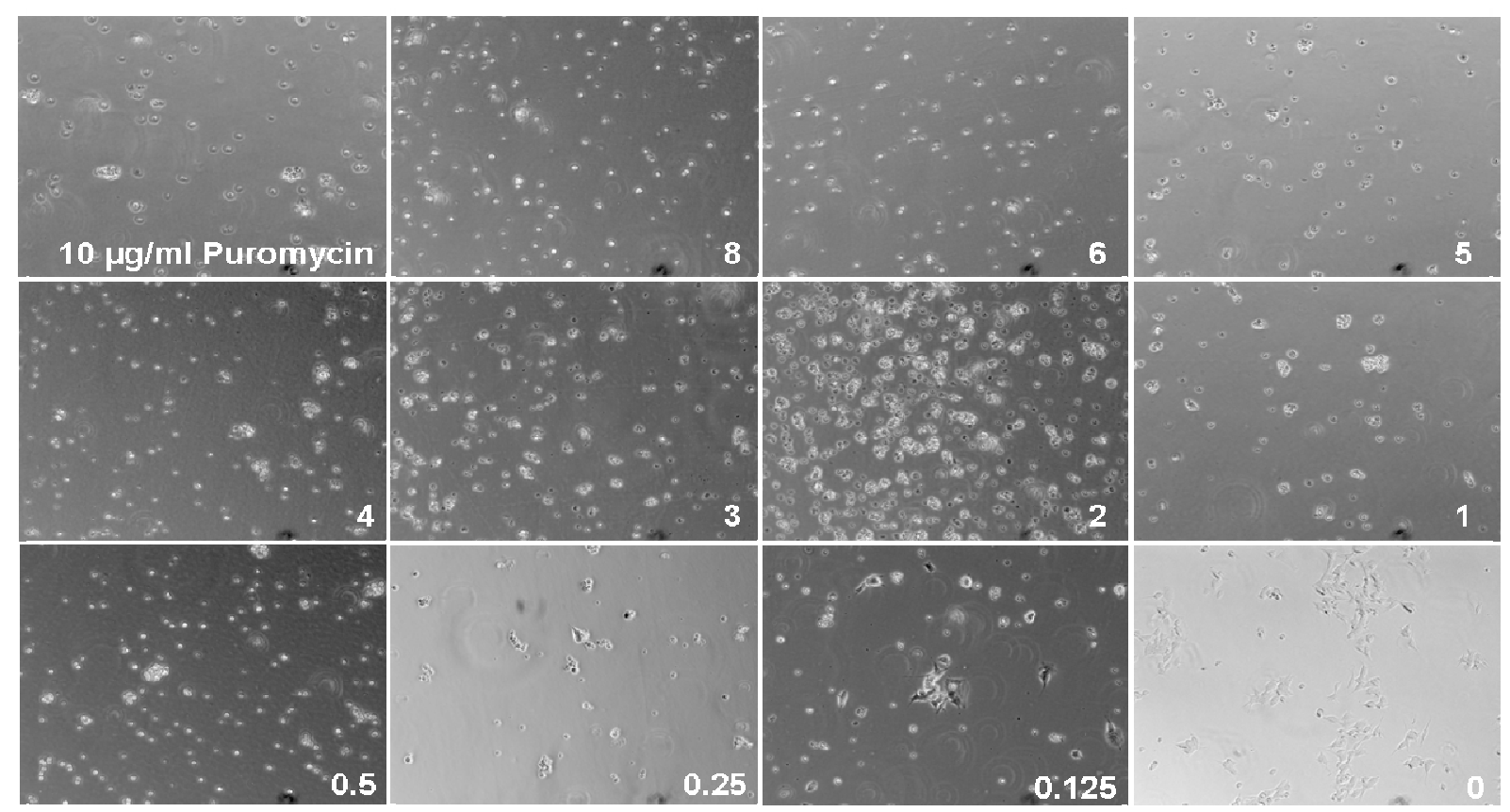

\section{Figure 3.5: Toxicity of puromycin in SH-SY5Y cells}

SH-SY5Y human neuroblastoma cells were treated with decreasing concentrations of puromycin $(10-0.125 \mu \mathrm{g} / \mathrm{ml})$ or were left untreated $(0 \mu \mathrm{g} / \mathrm{ml})$ for $4 \mathrm{~d}$. The puromycin concentrations in $\mu \mathrm{g} / \mathrm{ml}$ are shown in white numbers in the lower right in each picture. The pictures are imaged by phase contrast microscopy at magnification of 400x.

The same experimental set-up was chosen to evaluate the minimal toxic concentration of puromycin for SH-SY5Y cells. In contrast to HpL3-4 cells, SH-SY5Y, cells showed already inhibition of growth after the addition of $0.125 \mu \mathrm{g} / \mathrm{ml}$ puromycin (figure 3.5). At $0.25 \mu \mathrm{g} / \mathrm{ml}$ puromycin, all cells were completely detached. Thus, successfully transduced SH-SY5Y cells were selected with $0.25 \mu \mathrm{g} / \mathrm{ml}$ puromycin. This concentration virtually excluded the survival of non-transduced cells, and metabolic side effects were not detected. The determined minimal toxic concentration in SH-SY5Y was more than one order of magnitude lower than for HpL3-4 cells. Consequently, the minimal toxic dose of a given antibiotic has to be evaluated empirically for each cell line. 


\subsubsection{Generation of transgenic PrP HpL3-4 and SH-SY5Y cell lines}

According to the transduction efficiency experiments, we chose to use polybrene assisted spinduction to generate PrP transgenic cell lines.

Purified particles were harvested and HpL3-4 and SH-SY5Y were infected with retroviral particles carrying the transgene for $\operatorname{PrP}$ of $H$. sapiens, M.fascicularis, $S$. sciureus, O. virginianus, EMC or EGFP, respectively, as a positive control for transduction (figure 3.6). Only when these cells showed EGFP expression $4 \mathrm{~d}$ post transduction (dpt), the puromycin selection of stably PrP expressing cells was accomplished. This was achieved by culturing in antibiotic selection medium for 7 consecutive days with $3 \mu \mathrm{g}$ puromycin for transduced HpL3-4 or $0.25 \mu \mathrm{g} / \mathrm{ml}$ for transduced SH-SY5Y.

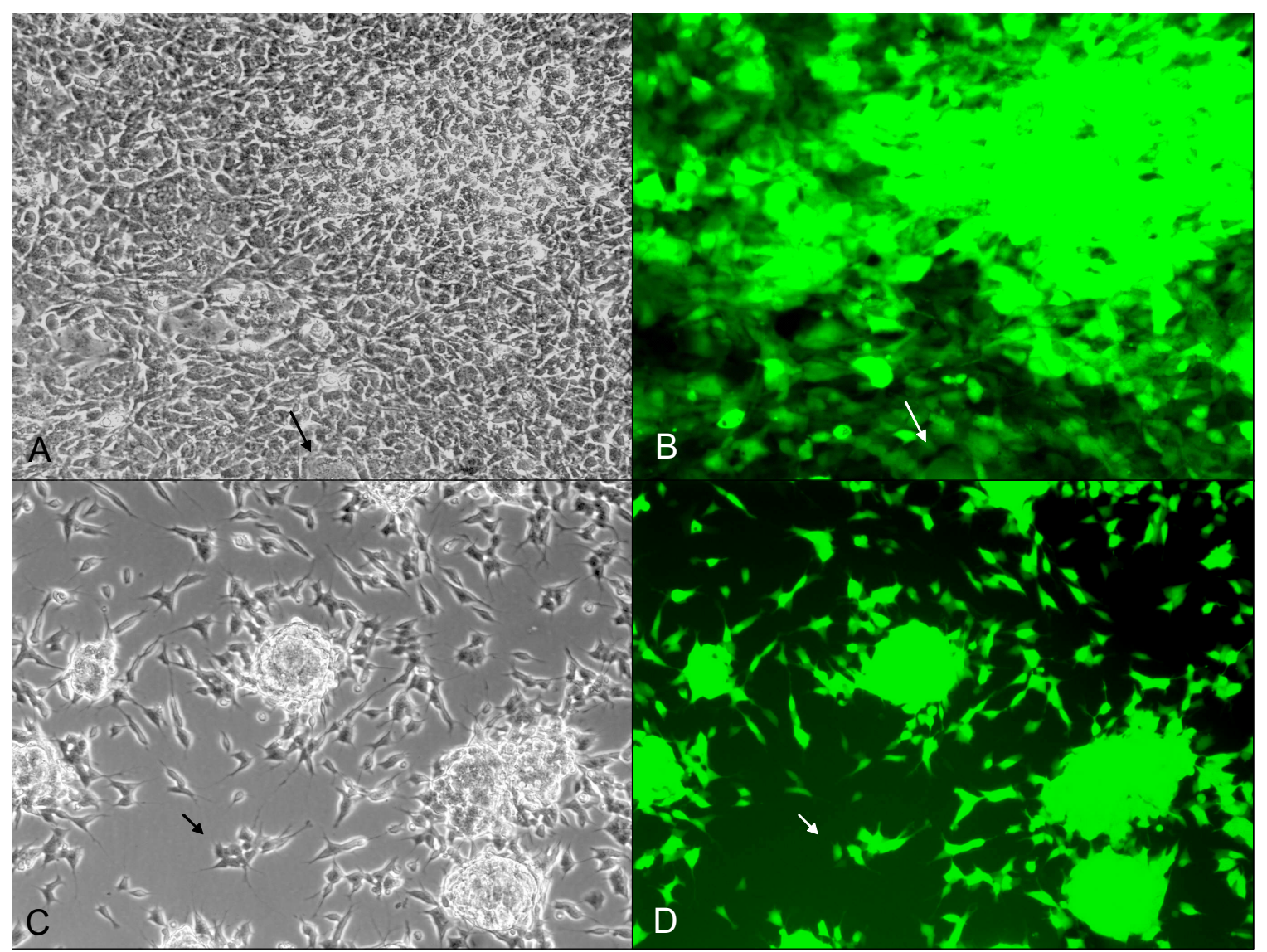

Figure 3.6: Expression of EGFP in HpL3-4 and SH-SY5Y cells after retroviral transduction

HpL3-4 (A,B) and SH-SY5Y (C, D) were transduced with retroviral particles carrying the EGFP transgene. EGFP expression was assessed by fluorescence microscopy (B, D) at 400x magnification. A, C: same image as B, D in phase contrast. 
After selection, cells were expanded and vitally deep-frozen. In total, PrP from four different species (and EMC control) was transduced into two different cell lines. The produced cell lines were named according to their transgene, i.e. $\operatorname{tg}($ species) PrP HpL3-4 or tg(species)PrP SH-SY5Y. Table 3.4 lists all 10 cell lines generated and characterised for prion infection experiments.

Table 3.4: PrP transgenic cell lines generated by retroviral transduction

\begin{tabular}{lll}
\hline denotation & PrP donor species & host cell line \\
\hline $\operatorname{tg}($ homo $)$ PrP HpL3-4 & H. sapiens & murine HpL3-4 \\
$\operatorname{tg}($ mafa $)$ PrP HpL3-4 & M. fascicularis & \\
$\operatorname{tg}($ sai $)$ PrP HpL3-4 & S. sciureus & \\
$\operatorname{tg}($ wtd $)$ PrP HpL3-4 & O. virginianus & \\
EMC HpL3-4 & empty vector control & \\
$\operatorname{tg}($ homo $)$ PrP SH-SY5Y & H. sapiens & \\
$\operatorname{tg}($ mafa $)$ PrP SH-SY5Y & M. fascicularis & \\
$\operatorname{tg}($ sai $)$ PrP SH-SY5Y & S. sciureus & \\
$\operatorname{tg}($ wtd $)$ PrP SH-SY5Y & O. virginianus \\
EMC SH-SY5Y & empty vector control & \\
\hline
\end{tabular}

\subsection{Molecular and biochemical characterisation of PrP transgenic HpL3-4 and SH-SY5Y cell lines}

Before starting infection experiments with PrP transgenic cell lines, we characterised the cell lines for the integration of PrP into the genome and expression of PrP. Furthermore, the surface presentation of $\operatorname{PrP}$ as a requirement for prion infection was evaluated (Borchelt, Taraboulos, and Prusiner, 1992; Feraudet et al., 2005; Taraboulos et al., 1992).

\subsubsection{Quantification of transgene integration sites into the host cell genome of HpL3-4 and SH-SY5Y cells}

To validate the efficacy of retroviral integration of transgenic PrP, gDNA was isolated from transduced and puromycin selected cells. The integration of PrP into the genome of 
HpL3-4 and SH-SY5Y cells was monitored by quantitative PCR. A cryo aliquot of each transgenic cell line (see table 3.4) was used to isolate gDNA (2.2.2.2). QPCR was performed with a SYBR green based assay (2.2.2.6.2) with transgene specific primer pairs (table 3.5). Due to nucleotide sequence homology of $H$. sapiens, $M$. fascicularis and O. virginianus identical primer pairs could be used (figure 3.7). Only for S. sciureus transgenic cells a different primer pair had to be selected (see table 3.5).

(490)

TaqPrP fw
H. sapiens
M. fascicularis
O. virginianus
S. sciureus
rcTaqPrP rev

TaqPrP fw

rcTaqPrP rev
ACCAAGTGTACTACAGGC

CCCAACCAAGTGTACTACAGGCCCATGG

CCCAACCAAGTGTACTACAGGCCTGTGG

CCCAACCAAGTGTACTACAGGCCAGTGG

CCCAGCCAAGTA-ATACTACA_AGCCCGTGG
[ ] (590)

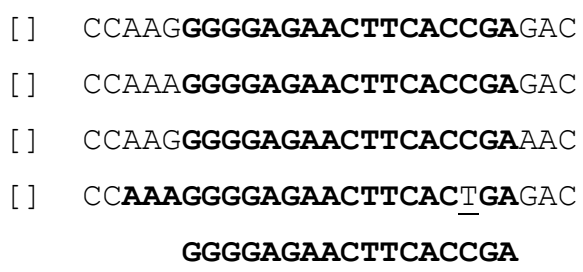

Figure 3.7: Nucleotide sequence alignment of $\operatorname{PrP}$ and primer pairs

DNA fragments of PrP from H. sapiens, M. fascicularis, O. virginianus and S. sciureus were aligned to the primer pair TaqPrP fw and the reverse complementary sequence of TaqPrP rev (rcTaqPrP rev). Identical sequences are in bold letters, mismatches are underlined. [] indicate omitted nucleotide regions.

The total number of gene copies per cell was evaluated to compare the transgene integration in all ten different cell lines (table 3.4). Absolute quantification of transgene from $H$. sapiens, $M$. fascicularis and $O$. virginianus was carried out by the standard curve methods using a serial dilution of pBABE-(mafa)PrP. Transgenic PrP from S. sciureus was quantified with pBABE-(sai)PrP to generate the standard curve (table 3.5).

Table 3.5: Primer pairs for qPCR with corresponding standardisation plasmid

\begin{tabular}{lll}
\hline species & primer pair & standard plasmid \\
\hline H. sapiens, M. fascicularis, & Taq PrP Fw, Taq PrP Rev, & pBABE-(mafa)PrP \\
O. virginianus & & \\
S. sciureus & diffSSc PrP Fw, diffSSc PrP Rev & pBABE-(sai)PrP \\
H. sapiens, M. fascicularis, & GAPDH Fw, GAPDH Rev & pGEM-TEasy-GAPDH \\
S. sciureus, O. virginianus & & \\
\hline
\end{tabular}

The abundance of the transgene was normalised to GAPDH DNA copies in each transgenic cell line. This was necessary since transgenic PrP was quantified with two 
different primer pairs. The absolute GAPDH copy number was evaluated by quantification to the plasmid pGEM-TEasy-GAPDH. The number of transgenes was calculated as tg copies per 100 copies GAPDH. A dilution factor (DF) was calculated from the amount of isolated DNA to DNA in one measured sample. To calculate the number of

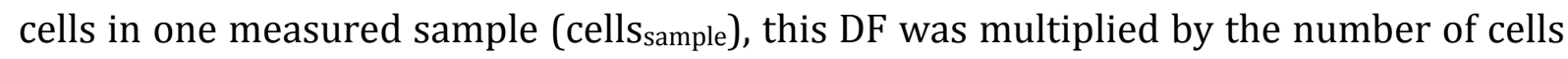
DNA isolated from. The ratio of absolute quantities of PrP/GAPDH of each cells sample

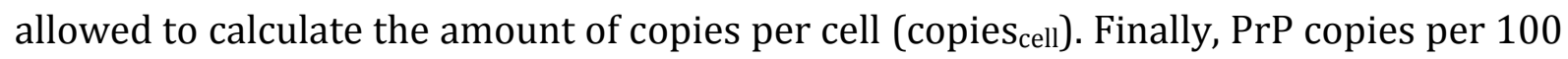
copies GAPDH was calculated by the ratio of tg copies cell $_{\text {to }}$ GAPDH copies cell $_{\text {multiplied }}$ by 100 . On the basis of transgene copies per GAPDH cell lines could be compared.

$$
\text { cell }_{\text {tube }}=\# \text { cells } \cdot \text { dilutionfactor } r_{R N A / g D N A} \quad \text { copies }_{\text {cell }}=\frac{\text { quantification }_{q P C R}}{\text { cell }_{\text {tube }}}
$$

transgene 100 copies GAPDH $=\frac{\text { transgene_copies }_{\text {cell }}}{\text { GAPDH_copies }} \cdot 100$

The PrP transgene integration sites were calculated for all produced HpL3-4 and SHSY5Y cell lines.

Due to their origin from $\mathrm{PrP}^{0 / 0}$ knockout mice, amplification of PrP in HpL3-4 cells is not possible. Thus, the signal derived from EMC HpL-3-4 cells was set as the background level at 0.1 transgene copies/100 copies GAPDH in all five $\operatorname{tg}(\mathrm{PrP}) \mathrm{HpL3}-4$ cell lines. GAPDH copies were compared, which revealed that all cell lines had a similar GAPDH copy number with approx. 3 copies per cell. Transgenic (sai)PrP HpL3-4 cells showed the highest number of integrated transgenes per 100 copies GAPDH, whereas the lowest copy number was measured for $\operatorname{tg}($ mafa)PrP HpL3-4 (figure 3.8).

In comparison, transgenic SH-SY5Y cell lines had a similar number of transgene copies/100 copies GAPDH, and the variation among the different transgenic cell lines was lower than among the tg HpL3-4 cell lines. In contrast to the tg HpL3-4 cell lines, in $\operatorname{tg}$ SH-SY5Y cell lines a smaller amount of GAPDH copies with a mean of 0.23 copies per cell was measured. The highest number of genomic PrP copies was present in $\operatorname{tg}(w t d) \operatorname{PrP}$ SH-SY5Y, the lowest number in $\operatorname{tg}($ sai $) \operatorname{PrP}$ SH-SY5Y. The number of transgene integration sites varied 3 fold at maximum. Due to its human origin, SH-SY5Y cells transduced with an empty virus (EMC) also amplified endogenous human PrP. 


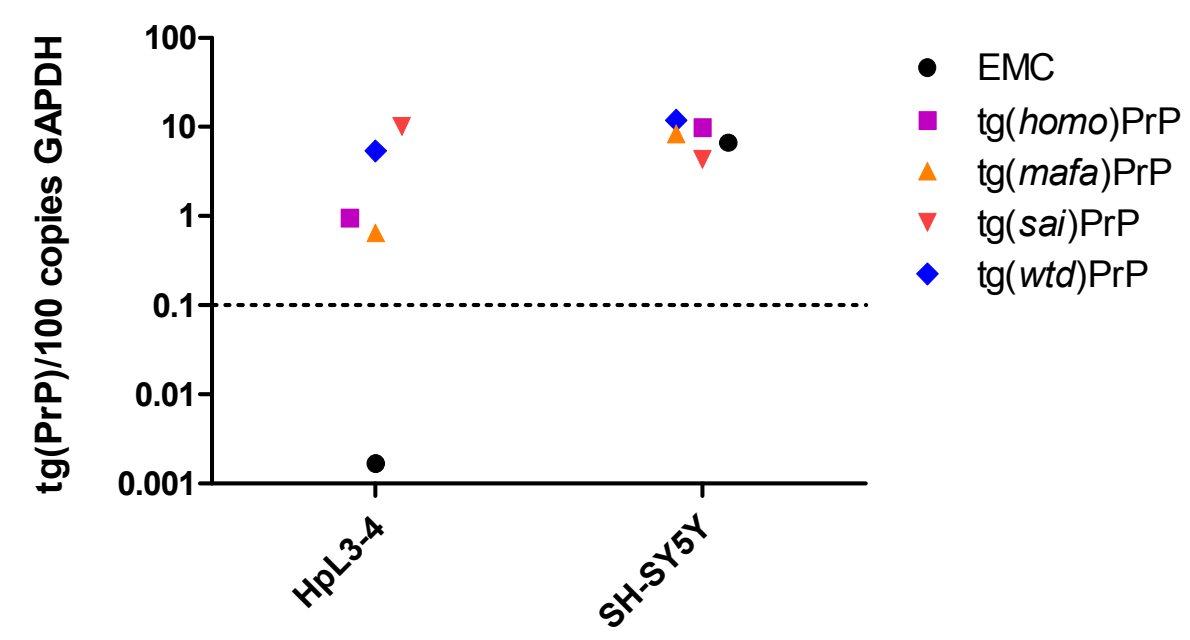

Figure 3.8: Number of transgenic PrP insertions in HpL3-4 and SH-SY5Y cells.

HpL3-4 and SH-SY5Y cells were retrovirally transduced with transgenic PrP from H. sapiens, M. fascicularis, S. sciureus, O. virginianus or EMC. Genomic DNA was isolated from each cell line and transgenes amplified by qPCR with sequence-specific primer pairs for PrP and GAPDH. The number of genomic insertions was calculated as ratio of tgPrP copies/100 GAPDH copies.

Independent of transgene donor or target cell species all cell lines had comparable copy numbers of respective transgenes. The transgene copy number among all cell lines varied in the range of 1-10 copies/100 copies GAPDH. This supports the robustness and reproducibility of the used transduction protocol.

\subsubsection{Expression of transgenic PrP in HpL3-4 and SH-SY5Y cells}

For a successful production of transgenic PrP not only the effective integration of the retroviral construct is necessary. The random insertion of a transgene into genomic DNA may alter the transcription rate of each insert due to epigenetic effects. Therefore, the relative transcription rate of each transgene was quantified. The RNA was then used for cDNA synthesis, and quantified by qPCR (figure 3.8). The qPCR was performed with sequence-specific primer pairs used to asses gDNA copy numbers in a SYBR green based assay (table 3.5). copy numbers were calculated as described.

All transgenic HpL3-4 cell lines showed comparable expression of GAPDH. Transgenic HpL3-4 cell lines contained 200-2500 copies of tg PrP/100 copies GAPDH . The highest mRNA expression level was detected for $\operatorname{tg}($ sai $) \operatorname{PrP}$ HpL3-4 with approximately 2500 
copies of $\operatorname{tgPrP} / 100$ copies GAPDH, the lowest for $\operatorname{tg}(w t d) \operatorname{PrP}$ HpL3-4. For the EMC cell line, the PrP signal was similar to the background as expected for $\mathrm{PrP}^{0 / 0}$ cells.

Comparable results were obtained for the transgenic SH-SY5Y cells. As for HpL-3-4, all SH-SY5Y cell lines showed consistent expression of GAPDH. The highest expression of PrP transgene on RNA level was detected for $\operatorname{tg}($ homo $) \operatorname{PrP}$ SH-SY5Y, the lowest for $\operatorname{tg}(w t d)$ PrP SH-SY5Y cells. The EMC SH-SY5Y cells did not show tg PrP or endogenous PrP mRNA expression above background level indicating that the PRNP gene is not transcribed in this neuroblastoma cell line. Compared to the HpL3-4 cells, a lower relative expression of PrP transgene was measured in transgenic SH-SY5Y cell lines. This is due to the normalisation dependent on the expression level of GAPDH in each cell line. Comparison of transgenic PrP without normalization to GAPDH revealed similar PrP RNA expression levels among all 10 HpL3-4 and SH-SY5Y cell lines.

In summary, all lines showed a constant and comparable level of transgene insertion and transcription, independent of the respective donor species or the target cell line. The used transduction procedure was highly efficient for the generation of stably $\operatorname{PrP}$ expressing cells. The quantification using qPCR offered the exact calculation of copy numbers per cells, which should correspond to the protein expression level, described in the next chapter. This is a necessary basis for the reproducibility of the following infection experiments.
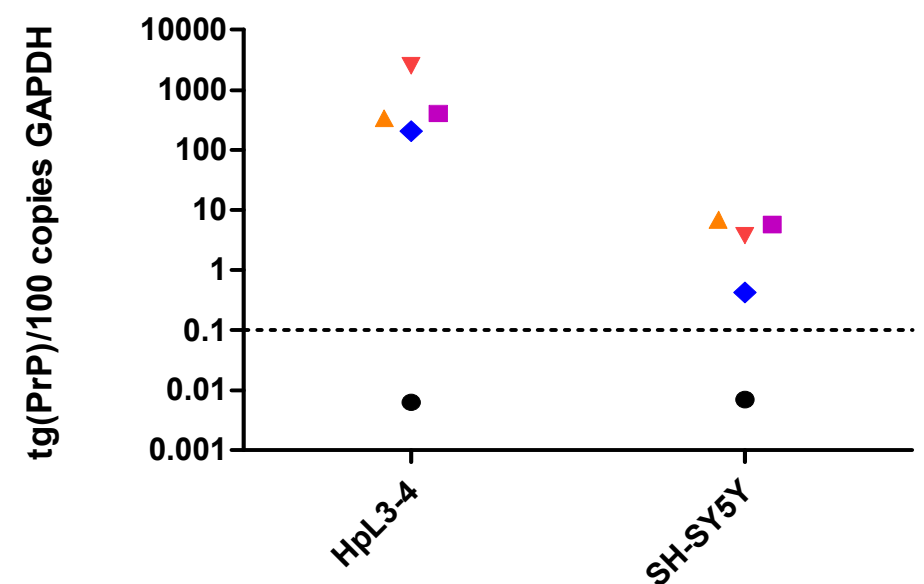

- EMC

- $\operatorname{tg}($ homo $) \operatorname{PrP}$

$\Delta \quad \operatorname{tg}($ mafa $) \operatorname{PrP}$

$\nabla \operatorname{tg}(s a i) \operatorname{PrP}$

$-\operatorname{tg}(w t d) \operatorname{PrP}$

Figure 3.8: Ectopic expression of transgenic PrP in HpL3-4 and SH-SY5Y cells HpL3-4 and SH-SY5Y cells were retrovirally transduced with transgenic PrP from H. sapiens, M. fascicularis, S. sciureus, O. virginianus or EMC. Total RNA was isolated, cDNA synthesised and qPCR was performed with PrP and GAPDH specific primer pairs. Copies were calculated as transgene copies PrP per 100 copies GAPDH. 


\subsubsection{Expression of prion protein in transgenic HpL3-4 and SH-SY5Y cell lines}

After comparative quantification of transgene integration and transcription levels in all generated cell lines, prion protein expression was measured to correlate transcription with translation. Cell lysates from all tgPrP HpL3-4 and SH-SY5Y cell lines were prepared (2.2.3.4) and samples separated on PAGE (2.2.3.5). A highly conserved PrP epitope among all species is a prerequisite for the comparative affinity of the used antibody to PrP. The membrane was incubated with mAb ICSM-18. The epitope of the mAb covers human PrP sequence AA 143-153 (GSDYEDRYYR) in helix 1 (Beringue et al., 2003). As shown in figure 3.9, this epitope is conserved in all species used except for serine 144 exchanged to asparagine in the macaque, sqirrel monkey and cervid prion protein. The high conservation of the epitope should allow comparative quantification.

(140) (150)
GSDYEDRYYR
IHFGSDYEDRYYRENM
IHFGNDYEDRYYRENM
IHFGNDYEDRYYRENM
IHFGNDYEDRYYRENM

\section{Figure 3.9: Alignment of ICSM-18 epitope}

A prion protein sequence part of $H$. sapiens, M. fascicularis, $S$. sciureus and $O$. viriginianus was aligned to the epitope of the mAb ICSM18. Identical amino acids within the epitope are bold letters, non-identical amino acids are underlined.

The blots were reprobed with anti- $\beta$-actin antibody as a loading control. Thus, comparability could be validated and relative quantification of prion protein was possible. For the HpL3-4 and SH-SY5Y cells equal amounts of $\beta$-actin were detected among all cell lines independently of transgene donor species (figure 3.10 middle panel) indicating comparable levels of protein lysate applied to each lane. Furthermore, $\operatorname{PrP}$ was detectable at comparable levels in most of the PrP overexpressing cell lines (figure 3.10 upper panel). The prion protein was detectable in PrP transgenic human, macaque, squirrel monkey and cervid cells. Prion protein expression was not detectable in EMC $\mathrm{PrP}^{0 / 0} \mathrm{HpL} 3-4$ cells transduced with empty retroviral expression vector demonstrating the specificity of the ICSM-18 mAb. In HpL3-4 cells the highest PrP expression was 
detectable for $\operatorname{tg}($ sai $)$ PrP cells, the lowest for $\operatorname{tg}(w t d)$ PrP cells. Transgenic SH-SY5Y did show transgene expression in $\operatorname{tg}($ homo $) \operatorname{PrP}, \operatorname{tg}(m a f a) \operatorname{PrP}$ and $\operatorname{tg}($ sai $) \operatorname{PrP}$ with the highest expression level for $\operatorname{tg}(m a f a)$ PrP. In $\operatorname{tg}(w t d) \mathrm{HpL3}-4$ cells the amount of PrP was markedly lower. Comparable results were obtained for $\operatorname{tg}(w t d) \operatorname{PrP}$ SH-SY5Y cells. The SH-SY5Y cell line does not have a $\mathrm{PrP}^{0 / 0}$ background as the HpL3-4 cell line and can express endogenous human $\mathrm{PrP}^{c}$. However, according to the RNA expression results with no amplification in the EMC SH-SY5Y cells the faint bands may result from unspecific binding. This is in line with the banding pattern that does not match to the banding pattern of the other tg PrP overexpressing SH-SY5Y cell lines. The banding pattern of $\operatorname{tg}(w t d)$ PrP SH-SY5Y appeared to be identical to that of EMC SH-SY5Y. Thus, the cells produce no or only low levels of white-tailed deer PrP PrP.

The prion protein has two glycosylation sites equivalent to a glycopattern with three predominant isoforms, the di-, mono- and un-glycosylated prion protein. Whether the glycosylation pattern depends on the donor species or the host cell line was further analysed. Both HpL3-4 and SH-SY5Y host cell lines, overexpressing transgenic PrPc from certain species, were investigated for their PrP glycosylation pattern. All transgenic $\mathrm{PrP}^{\mathrm{c}}$ within one host cell line showed a comparable glycopattern. In comparison, both neuronal cell lines (HpL3-4 and SH-SY5Y) differed marked in the distribution of di-, mono and unglycosylated PrP isoforms independent of the transgene donor species, but on the recipient cell line species. In the HpL3-4 cell lines the di- and mono glycosylated isoform is predominant, whereas in the SH-SY5Y cells the diglycosylated band is most abundant (see figure 3.10). Either the different host cell species or the different cell background can cause this for both HpL3-4 (murine hippocampus neurons) and SHSY5Y (human neuroblastoma) leading to different cellular processes such as posttranslational modification.

The total protein expression was further compared to RNA expression in each cell line. Prion protein expression was normalised to $\beta$-actin expression and PrP RNA copies to GAPDH copies. In general, the transgenic $\mathrm{PrP}^{\mathrm{c}}$ protein expression correlates well with that of RNA (figure 3.10 lower panel). Cell lines with low PrP RNA copy number expressed less prion protein. In summary, Western blot analysis confirmed the expression of transgenic prion protein, a pre-requisite for the infection of the transgenic cell lines with prion-infectious agents (Bueler et al., 1993). Additionally, the surface 
presentation of $\operatorname{PrP}$ is necessary to successfully infect cells with prion-infectious agent (McNally, Ward, and Priola, 2009), which was then examined.

A

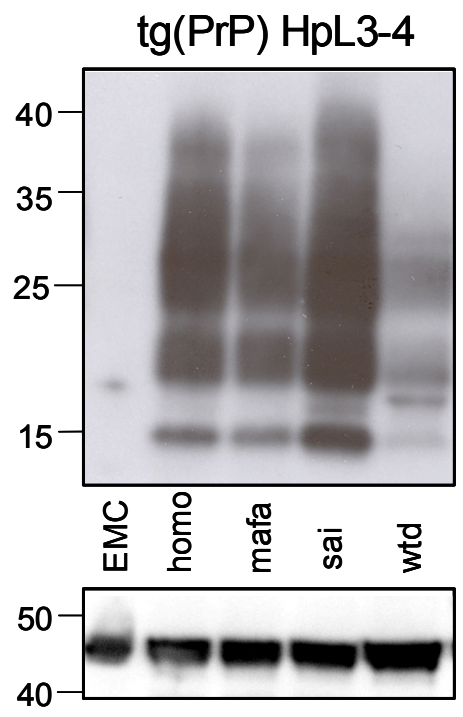

$\operatorname{PrPc}$

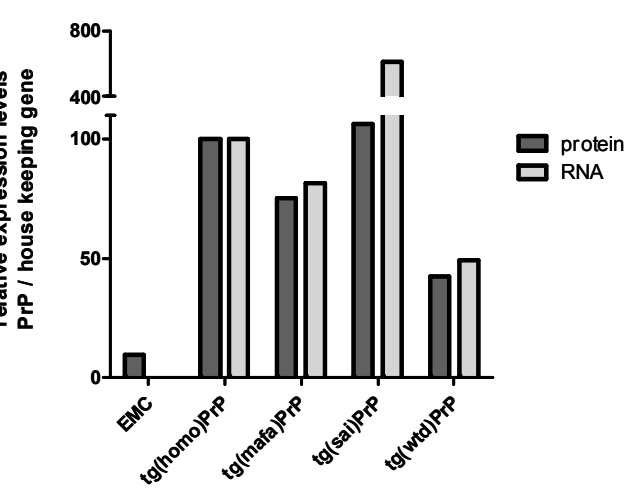

B
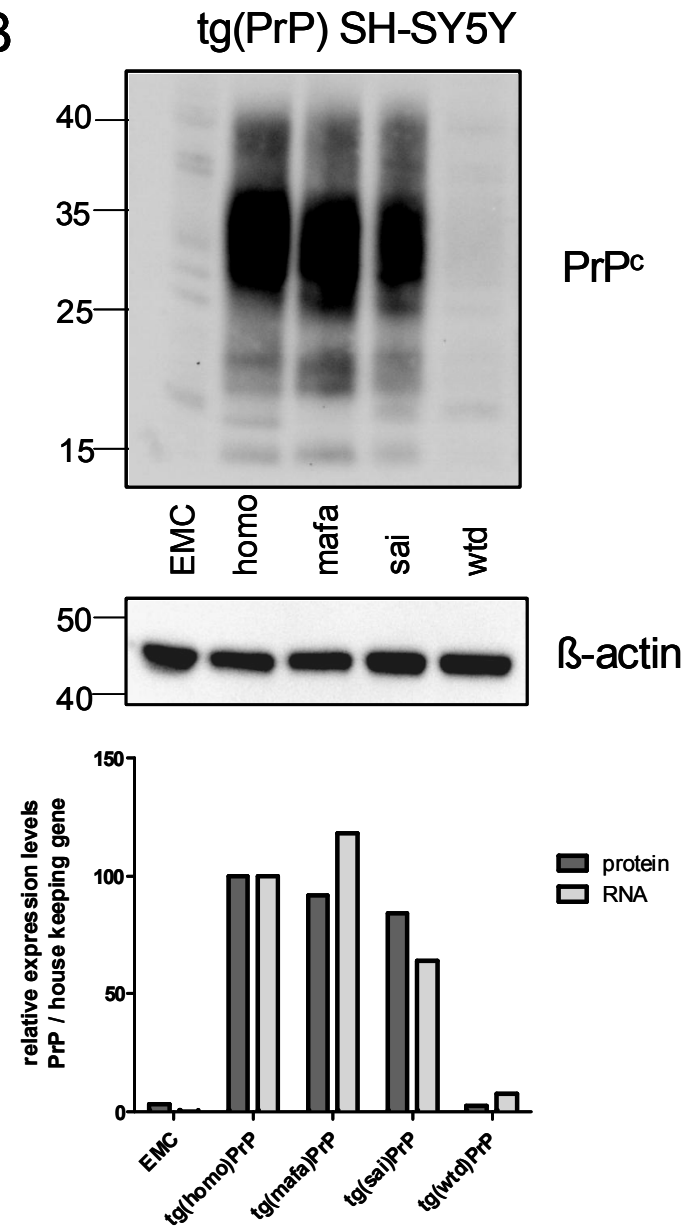

Figure 3.10: Prion protein expression in transgenic HpL3-4 and SH-SY5Y cells

Cell lysate was prepared from transgenic (homo)PrP-, (mafa)PrP-, (sai)PrP-, (wtd) PrP and EMC HpL3-4 (A) and SH-SY5Y (B) cells and analysed by Western blot. Prion protein was detected with mAb ICSM-18. The same membrane was probed with anti- $\beta$-actin Ab as loading control (middle panel). The lower panel shows the correlation of protein to RNA expression in all cell lines ( $\mathrm{x}$-axis) as relative expression levels of PrP to the respective housekeeping gene ( $\beta$-actin for protein expression, GAPDH for RNA expression, $\mathrm{y}$-axis). The molecular weight in $\mathrm{kDa}$ is depicted on the left. 


\subsubsection{Surface presentation of transgenic PrP in HpL3-4 and SH-SY5Y cells}

\subsubsection{Titration of primary anti-PrP antibody in $\operatorname{tg}(\mathrm{mo}) \operatorname{PrP} \mathrm{HpL3}-4$ cells}

Although transgenic prion protein expression was confirmed by Western blot and comparable levels of protein were detected among the generated cell lines, the surface abundance of the protein had to be compared. The presentation of the protein at the cell surface by binding via a GPI anchor to the cell membrane, is a requirement for the infection with PrPres (Klingeborn et al., 2011; McNally, Ward, and Priola, 2009; Priola and McNally, 2009). A sophisticated method detecting PrP surface presentation was chosen. Flow cytometry allows the measurement of cell surface protein abundance and subsequent quantitative comparison of all transgenic PrP cell lines. A set of anti-prion antibodies was chosen to exclude species-specific and epitope-dependent effects. The mAb 3B5 binds to the octarepeat region of human PrP at AA 62-91, an epitope highly conserved among all analysed species. Due to its repetition within the prion primary structure it can bind manifold to the protein depending on the exposition of the domain in the tertiary or quaternary structure. The mAb 12F10 binds to the epitope of human $\operatorname{PrP}$ at AA 143-157 located in the non-structured region N-terminally of helix 2. For macaque, squirrel monkey and cervid PrP two AA are exchanged within mAb 12F10 epitope (figure 3.11). One additional amino acid exchange in squirrel monkey $\operatorname{PrP}$ (serine to asparagines) and in mouse PrP (tyrosine to tryptophan) may interfere with antibody binding. In addition, the anti-prion mAb 11C6 was also used although the epitope is unknown since peptide mapping of the epitope failed so far (D. Motzkus, personal communication). 


\begin{tabular}{|c|c|c|c|}
\hline & $(70)$ & & $(140)$ \\
\hline $\mathrm{mAb} 3 \mathrm{~B} 5$ & GWGQPHGGGWG & mAb $12 \mathrm{~F} 10$ & GSDYEDRYYRENMHR \\
\hline H. sapiens & GGWGQPHGGGWG $Q P H$ & & IHFGSDYEDRYYRENMHRY \\
\hline M. fascicularis & GGWGQPHGGGWG $Q P H$ & & IHFGNDYEDRYYRENMYRY \\
\hline O. virginianus & GGWGQPHGGGWGQPH & & IHFGNDYEDRYYRENMYRY \\
\hline S. sciureus & GGWGQPHGGGWGQPH & & IHFGNDYEDRYYRENMYRY \\
\hline M. musculus & GTWGQPHGGGWGQPH & & IHFGNDWEDRYYRENMYRY \\
\hline
\end{tabular}

Figure 3.11: Conservation of monoclonal antibody epitopes among different species

Selected sequences of the prion protein from $H$. sapiens, M. fascicularis, $S$. sciureus, $O$. virginianus and $M$. musculus were aligned to the epitopes of the mAbs 3B5 (left) and 12F10 (right). Identical sequences are in bold, variations are underlined.

To detect PrPc, also referred to as CD 230 on the surface of transgenic cell lines, generated cell lines were examined by flow cytometry (2.2.3.6). Therefore, $\operatorname{tg}(m o) \operatorname{PrP}$ HpL3-4 cells (kindly provided by Prof. I. Vorberg, DZNE Bonn) were detached, pelleted and fixed for flow cytometry. First, the cell population was defined to exclude background signals derived from cell debris. The densest population was gated in the forward (FSC) and sideward scatter (SSC). This characterises all cells according to their size (FSC), and granularity (SSC, see figure 3.12-A). Next, this densest population was selected to further discriminate single signals from doublets (figure 3.12-B), which could result from cell clusters. Signals from doublets would interfere in subsequent quantification of surface PrP. Here, as well, the densest population was further depicted (figure 3.12-C). The resulting cell population was investigated for its autofluorescent emission in the fluorescence channel later used for the detection of cell surface-bound CD230. This population was finally plotted as a histogram, showing cell counts against fluorescence intensity (figure 3.12-D). This illustration was chosen to easily visualise an increase in fluorescence leading to a shift from autofluorescence to actual detection of cell surface-associated PrP. 
A

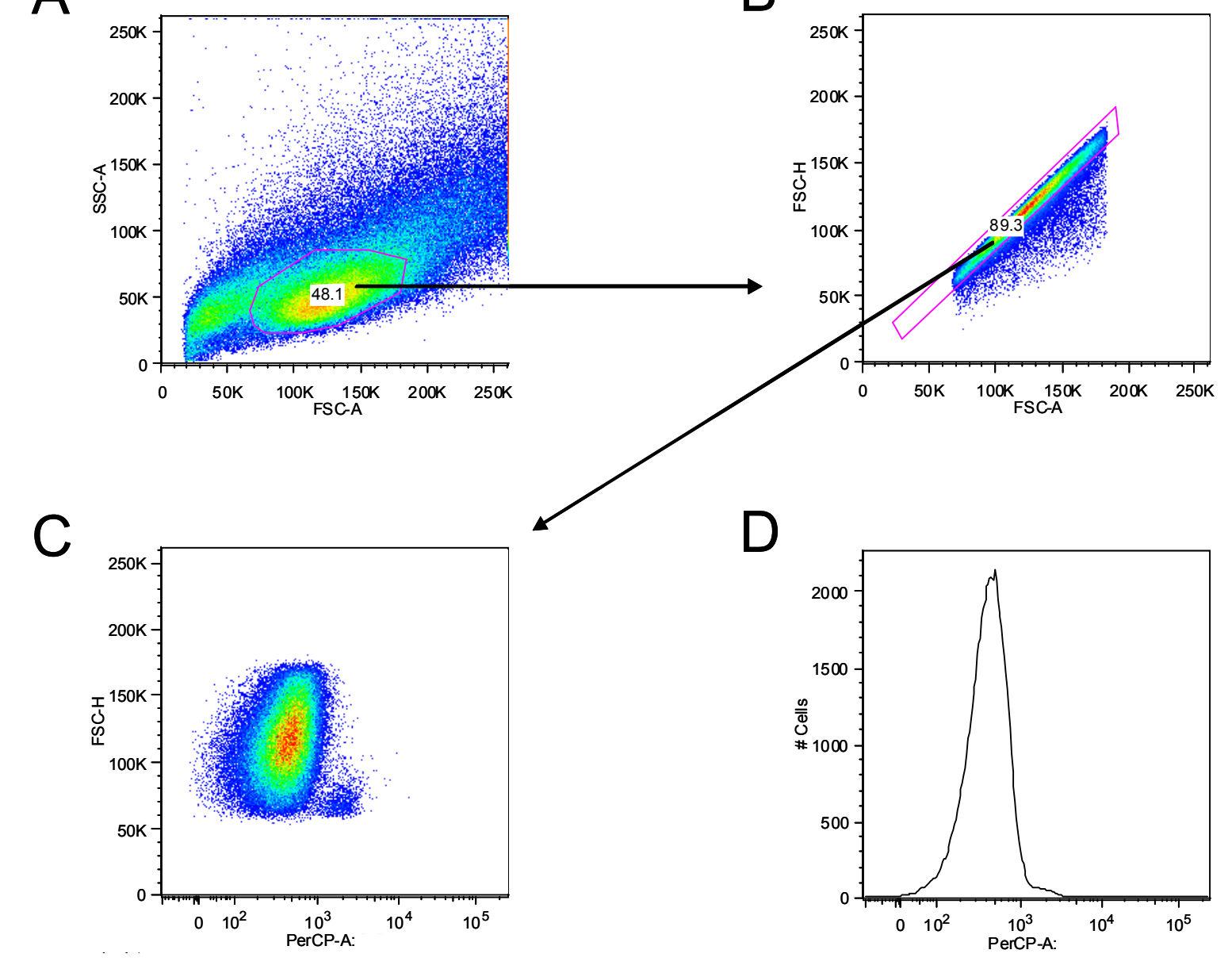

B

Figure 3.12: Flow cytometry gating pattern of HpL3-4 cells

Transgenic ( $m o$ ) PrP HpL3-4 cells were detached and analysed by flow cytometry.

A shows all signals in FSC and SSC with the gate for the densest population (purple line with \% of total signal counts). B shows gated signals from A in FSC-A versus FSC-H with the selection of single signals (purple line with \% of selected signals from the gate in A). C shows the resulting fluorescent singlet cell population (PerCP-A), also depicted in $\mathbf{D}$ as a histogram for cell counts against fluorescence. Dots represent particles according to their density (blue<green<yellow $<$ red). Arrows indicate the gating sequence.

Next, PrP cell surface abundance was determined with a prion specific antibody. First, a suitable antibody concentration had to be evaluated. First the primary anti-PrP antibody 12F10 was chosen directed against the human PrP epitope AA 143-157. The suitability of this antibody for flow cytometry was already shown for blood fraction analysis of BSE-infected macaques (Holznagel et al., 2010). The cell population was characterised and autofluorescence was defined. To detect tg PrP on the surface, $\operatorname{tg}(m o) \operatorname{PrP}$ HpL3-4 cells were detached, pelleted and incubated with serial dilutions of mAb 12F10. 


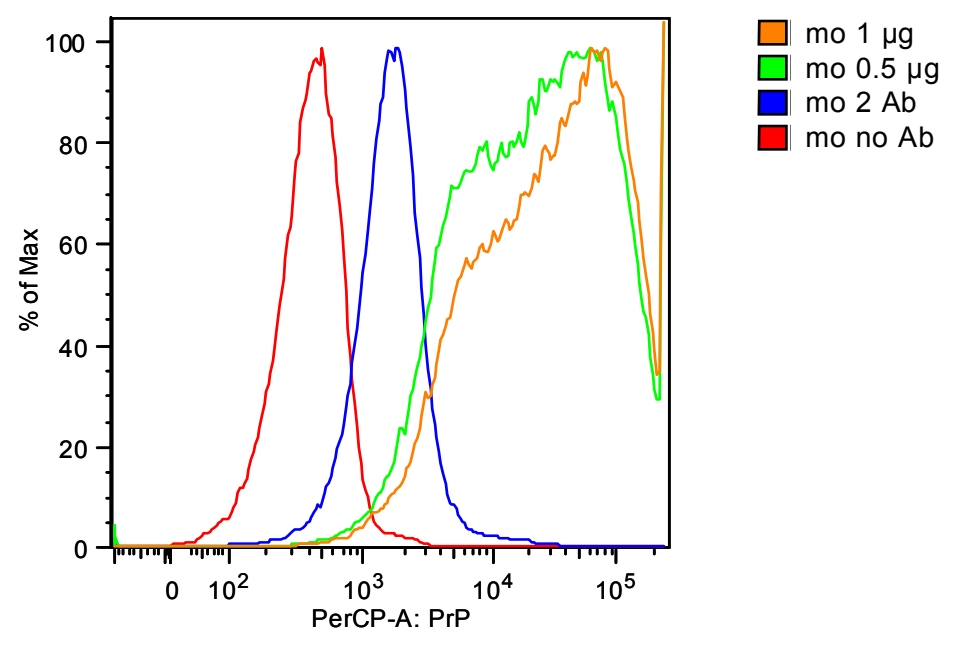

Figure 3.13: Specific detection of murine prion protein on $\operatorname{tg}(\mathrm{mo}) \mathrm{PrP}$ HpL3-4 cells by flow cytometry

Transgenic (mo)PrP HpL3-4 cells were detached and incubated without antibodies (red), secondary PE-Cy5 coupled antibody alone (blue) or with 0.5 (green) or $1 \mu \mathrm{g} / 10^{6}$ $\mathrm{mAb} 12 \mathrm{~F} 10$ (orange). The established gating pattern (see text) was employed. The histogram depicts the relative frequency of all measured cells (\% of Maximum), plotted against the logarithm of fluorescence intensity (PerCP-A: PrP).

The sample was further incubated with a secondary anti-mouse antibody coupled to the tandem conjugate PE-Cy5. Unspecific binding of secondary antibody was quantified by omission of primary antibody. This revealed an increasing shift in fluorescence intensity when compared to the control lacking both antibodies, indicating that the secondary antibody itself has a low capacity to bind unspecifically to $\operatorname{tg}(m o) \operatorname{PrP}$ HpL3-4 cells.

The flow cytometric analysis with mAb 12F10 detected cell surface-attached PrP on $\operatorname{tg}(m o) \operatorname{PrP} H p L 3-4$ cells for both concentrations $0.5 \mu \mathrm{g} / 10^{6}$ cells and $1 \mu \mathrm{g} / 10^{6}$ cells (figure 3.13). Since the signal-to-noise-ratio was best at $1 \mu \mathrm{g} 12 \mathrm{~F} 10 / 10^{6}$ cells, this concentration was chosen for further quantitative detection of transgenic PrP by flow cytometry.

\subsubsection{Influence of cell detachment procedures on the detectability of surface bound transgenic PrP}

The detachment procedure could influence the detectability of $\operatorname{PrP}$ on the cell membrane of the adherent transgenic HpL3-4 and SH-SY5Y cells. This was examined by flow cytometry according to the established protocol. Depending on the used detachment procedure, surface proteins can be removed or degraded by enzymatic activity. We compared three different enzymatic reagents (trypsin, accutase and tryple 
select) according to the manufacturers' instructions with mechanical scraping of cells from the surface of the culture dishes. Accutase and tryple select reagents should be gentle detachment procedures especially useful for the detection of surface-proteins by flow cytometry. We used the same cell line as above $(\operatorname{tg}(m o) \operatorname{PrP} \mathrm{HpL3}-4)$, together with the established experimental conditions and gating schemes (3.3.4.1). If detachment procedure does not influence PrP detection, the histograms should not differ. The different reagents had a profound influence on the detectability of PrP (see figure3.14). The loss of PrP signal was equivalent to the difference between the signals derived from treated cells compared to the non-treated scraped cell population. After mechanical scraping the highest PrP surface presentation was detectable. This procedure was suited best to analyse surface PrP in this cell line. After enzymatic detachment by trypsin, less surface PrP was detectable and even less remained after tryple select or accutase. These results are in conflict with the suppliers' statements. Thus, for further flow cytometry experiments detachment via scraping was chosen.

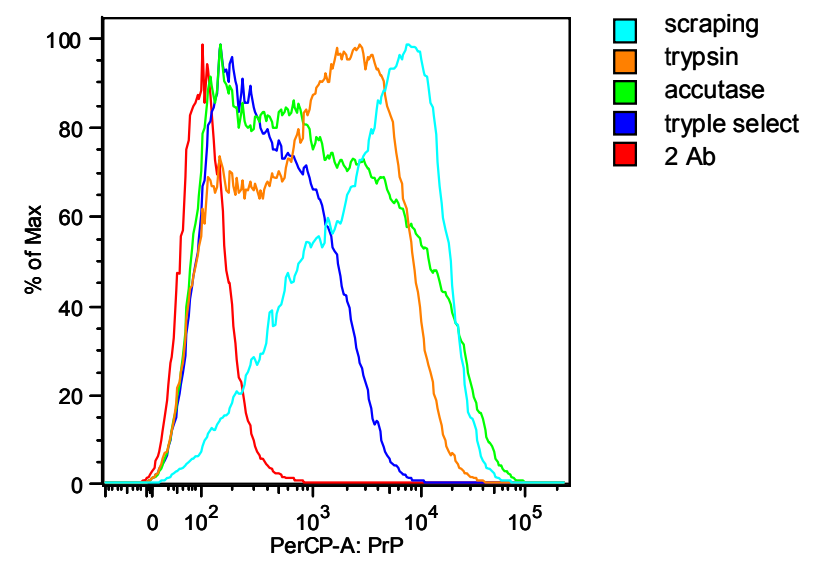

Figure 3.14: Influence of cell detachment procedures on the detection of surfacebound PrP

Transgenic (mo)PrP HpL3-4 cells were detached by four different detachment techniques (tryple select, accutase, trypsin and scraping). Cells were incubated with anti-prion mAb 12F10, and PE-Cy5 as secondary $\mathrm{Ab}$ and then measured by flow cytometry following the gating pattern established (figure 3.12). The $\mathrm{x}$-axis depicts the relative fluorescence intensity in arbitrary units in a logarithmic scale (PerCP-A: PrP); the $y$-axis the relative frequency of all measured cells in percentage ( $\%$ of Max). 


\subsubsection{Quantification of surface-bound PrP in transgenic HpL3-4 and SH-SY5Y cell lines}

All transgenic HpL3-4 and SH-SY5Y cell lines were compared for surface PrP presentation. All transgenic HpL3-4 and SH-SY5Y cell lines were incubated with $1 \mu \mathrm{g} / \mathrm{x} 10^{6}$ cells of the mAb $12 \mathrm{~F} 10$. All transgene donor species were tested (H. sapiens, $M$. fascicularis, S. sciureus, O. virginianus) and compared to the non-PrP expressing EMC cells.

First, the EMC HpL3-4 cell line was tested. Due to the lack of PrP expression in HpL3-4 $\mathrm{PrP}^{0 / 0}$ cells, the unspecific binding of both primary and secondary antibody defines the detection limit of the procedure. Using mAb 12F10, CD230 was detected on $\operatorname{tg}(w t d) \operatorname{PrP}$ HpL3-4 cells indicated by a slight shift in fluorescence intensity when compared to the EMC cells (figure 3.15-A). PrP surface abundance was detectable but low, concurrent with the RNA and Western blot data for this cell line showing low expression of cervid PrP (figure 3.15.-A). Human, macaque and squirrel monkey PrP transgenic cell lines showed higher signal intensities also in line with the transcription and Western blot results. Interestingly, FACS analysis detected two distinct signal peaks (arrows exemplarily in $\operatorname{tg}($ sai $)$ PrP HpL3-4 cells in figure 3.15-A). These distinct signal peaks most probably result from different cell populations harbouring varying amounts of PrP on their cell surface.

One possibility is that this may have been caused by the gene delivery method. Retroviral transduction leads to a random integration of the transgene and a heterogeneous cell population with different transcription and translation performances. Alternatively, one single cell can be transduced several times resulting in higher gene density and expression of PrP. However, this would rather broad the peak areas than producing two distinct peaks. Although puromycin selection was accomplished, the antibiotic treatment only selects for all transduced cells. Moreover, the distinct populations may be explained by the cell cycle when a dividing cell has the PrP surface amount apportioned for two cells. These two different populations would not have been detected on genomic, transcriptional or protein expression level assessed by qPCR and Western blot. These techniques examine cellular populations as a whole.

A further possibility is that the distinct populations of PrP presenting cells might be caused by the choice of antibody. As indicated in figure 3.11 the epitope of the antibody slightly differs among the species. In addition, staining of the cells for flow cytometry 
analysis allows binding of the antibodies to native PrP, thus the appearance of different cell populations may also be due to native binding partners of PrP that are differently expressed. For human, macaque and squirrel monkey cells, the first population presents more surface PrP than non-PrP expressing EMC cells. In the second population of tg (homo)PrP, $\operatorname{tg}(m a f a) \operatorname{PrP}$ and $\operatorname{tg}($ sai $) \operatorname{PrP}$ cells, higher amounts of surface-presented $\operatorname{PrP}$ were detected.

To examine the potential antibody effect, a panel of $\mathrm{mAb}$ raised against different prP epitopes was employed. Therefore, two additional mAbs (mAb 3B5 and 11C6) were tested for surface PrP presenting cells according to the established protocol and gating pattern. Prior to use, we confirmed that, for mAb 3B5 and 11C6, $1 \mu \mathrm{g} / 10^{6}$ cells was also suitable to detect surface PrP in the transgenic cell lines (data not shown).
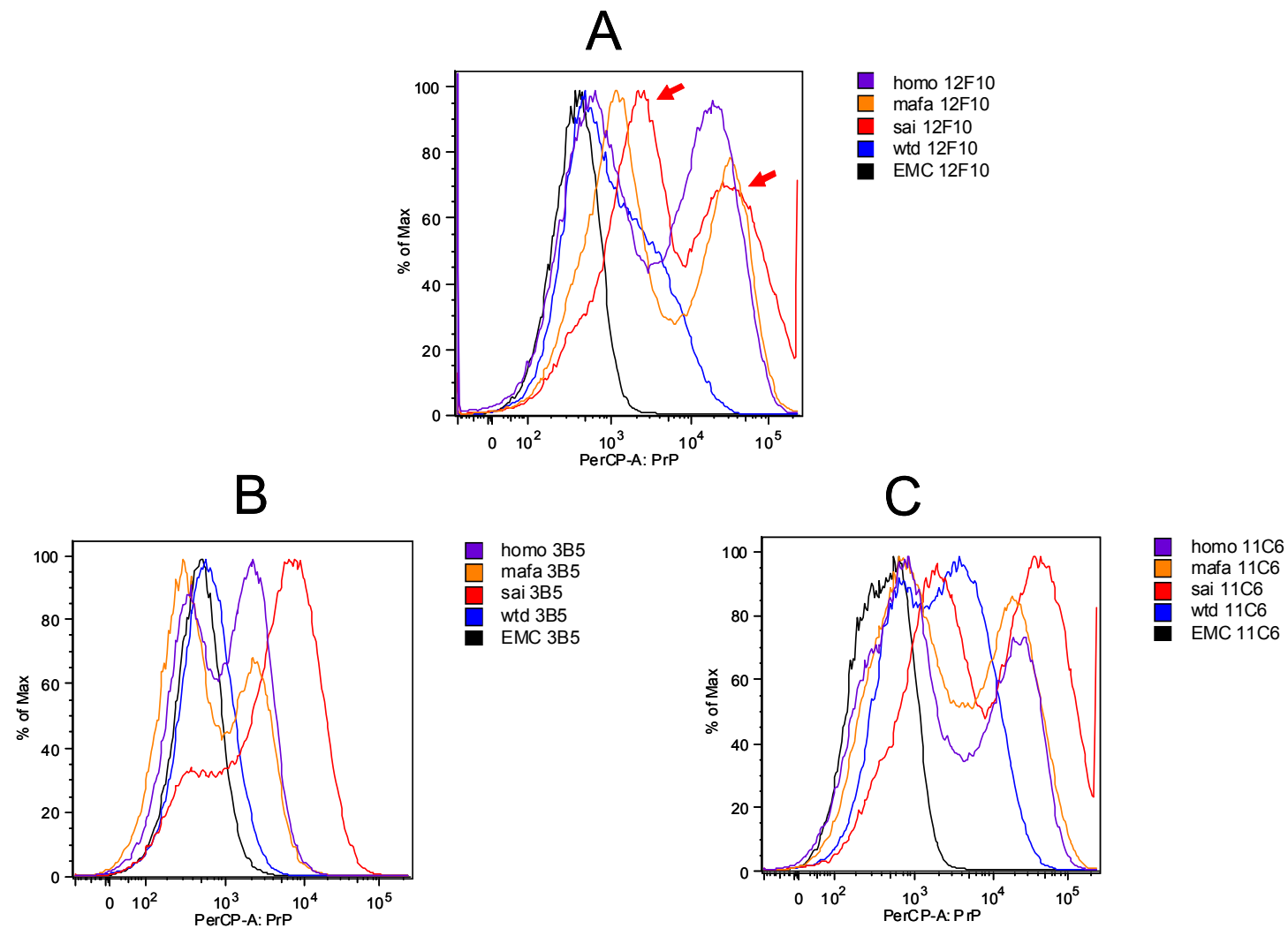

Figure 3.15: Surface abundance of prion protein in transgenic HpL3-4 cells

Transgenic (homo)PrP-, (mafa)PrP-, (sai)PrP-, (wtd) PrP- and EMC HpL3-4 cells were detached by scraping from the cell culture dish. Cells were incubated with primary antiprion protein mAbs 12F10 (A), 3B5 (B), 11C6 (C). All signals derived by omission of the primary antibodies were lower than signals on non-PrP expressing EMC cells (data not shown). The secondary antibody was PE-Cy5 labeled. The x-axis shows fluorescence intensity units, the $y$-axis the relative cell count in percent. Red arrows indicate peaks of different populations in $\operatorname{tg}($ sai $)$ PrP cells (see text). 
Again, all HpL3-4 cell lines showed two distinct populations of PrP surface abundance. However, on $\operatorname{tg}(w t d) \operatorname{PrP}$ HpL3-4 cells PrP was only slightly detectable. The mAb 11C6 revealed comparable PrP surface abundance for all tested cell lines (figure 3.15-C) apart from cervid PrP cells where a cell population with high PrP expression compared to EMC cells was discriminated. But in comparison to human, macaque and squirrel monkey PrP cells, abundance of PrP was lower in cervid PrP expressing cells as confirmed with three different antibodies.

Table 3.6 summarises the relative cell abundance of PrP as mean fluorescence intensity calculated on the PerCP signal in all HpL3-4 cell lines analysed with the antibodies, used for the detection of cell surface bound transgenic PrP. Comparison of cervid PrP presenting cells with EMC cells indicated that only mAbs $11 \mathrm{C} 6$ and 3B5 discriminate by one order of magnitude between non-expressing (EMC) and PrP expressing cell. Furthermore, human and macaque cells showed highly comparable PrP surface presentation levels. The highest PrP surface abundance was measured in squirrel monkey PrP cells. In general, the detection of PrP in transgenic cell lines was one order of magnitude higher by using mAbs $11 \mathrm{C} 6$ and 3B5 as compared to mAb $12 \mathrm{~F} 10$.

Table 3.6: Mean fluorescence intensity for transgenic PrP presenting HpL3-4 cells

\begin{tabular}{llll}
\hline cell line & mAb 12F10 & mAb 11C6 & mAb 3B5 \\
\hline $\operatorname{tg}($ homo $)$ PrP HpL3-4 & $1.57 \times 10^{3}$ & $1.00 \times 10^{4}$ & $1.17 \times 10^{4}$ \\
$\operatorname{tg}($ mafa $)$ PrP HpL3-4 & $1.33 \times 10^{3}$ & $1.16 \times 10^{4}$ & $1.53 \times 10^{4}$ \\
$\operatorname{tg}($ sai $)$ PrP HpL3-4 & $7.46 \times 10^{3}$ & $3.23 \times 10^{4}$ & $2.76 \times 10^{4}$ \\
$\operatorname{tg}($ wtd $)$ PrP HpL3-4 & $7.50 \times 10^{2}$ & $4.27 \times 10^{3}$ & $2.30 \times 10^{3}$ \\
EMC HpL3-4 & $6.17 \times 10^{2}$ & $5.44 \times 10^{2}$ & $4.89 \times 10^{2}$ \\
\hline
\end{tabular}

To examine, if the results obtained from tg HpL3-4 cell lines may attribute to the transgenic PrP expression, the tg SH-SY5Y cell lines were analysed by flow cytometry with the established PrP surface detection conditions. In contrast to the HpL3-4 cell line, the human SH-SY5Y cell line encodes for endogenous prion protein. But according to the results obtained for RNA (3.3.2) and protein expressions (3.3.3), PrP should not be detectable at the surface of these cells. A new gating pattern was designed for the size, granularity and autofluorescence of SH-SY5Y cells (data not shown). As shown in 
figure 3.16, presentation of PrP on transgenic SH-SY5Y was comparable to that found on HpL3-4 cells. The PrP signals on SH-SY5Y cells obtained with mAbs 12 F10 (figure 3-16A) and 3B5 (figure 3-16-B) were comparable. Thus, the antibodies are suitable for further analyses on the different transgenes. Again, the shape of histograms indicated a heterogeneous population of PrP presenting cells as already observed for transgenic HpL3-4 cells. MAb 11C6 (figure 3.16-C) confirmed the results obtained earlier with mAbs 12F10 and 3B5. However, the antibody had an unfavourable signal-to-noise-ratio in EMC SH-SY5Y cells. Since directed against a human epitope it could cross-react with other cell surface proteins on SH-SY5Y at the used concentration.
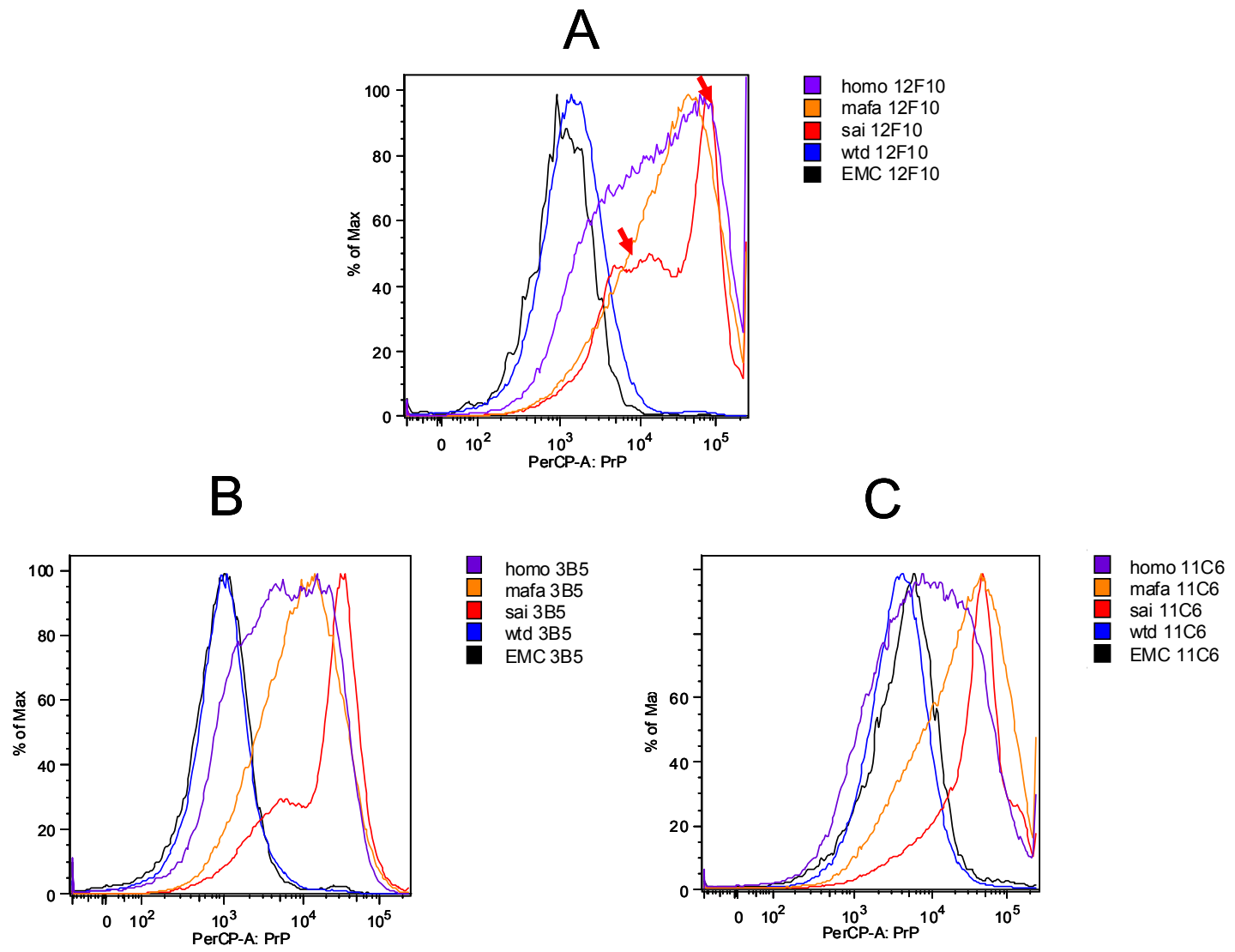

\section{Figure 3.16: Surface abundance of prion protein in transgenic SH-SY5Y cells}

Transgenic (homo)PrP-, (mafa)PrP-, (sai)PrP-, (wtd)PrP- and EMC SH-SY5Y cells were detached by scraping from the cell culture dish. Cells were incubated with primary antiprion protein mAbs 12F10 (A), 3B5 (B), 11C6 (C). All signals derived by omission of the primary antibodies werelower than signals on non-PrP expressing EMC cells (data not shown). The secondary antibody was coupled to PE-Cy5. The x-axis shows fluorescence intensity units (PerCP-A: PrP), the y-axis the relative cell count in percent ( $\%$ of Max). 
Table 3.7 summarises the surface results of the transgenic SH-SY5Y cell lines with corresponding antibodies shown by mean fluorescence intensity values. The results are highly consistent to the results obtained in HpL3-4 cells where human and macaque PrP expressing cells showed comparable PrP surface expression. Furthermore, squirrel monkey transgenic cells had the highest PrP surface density. Cervid PrP was not observed on tg SH-SY5Y cells but the mAb 3B5 gave a very low abundance of PrP signal comparable to that of EMC cells.

Table 3.7: Mean fluorescence intensities for transgenic PrP presenting SH-SY5Y cells

\begin{tabular}{llll}
\hline cell line & $\mathrm{mAb} 12 \mathrm{~F} 10$ & $\mathrm{mAb} 11 \mathrm{C} 6$ & $\mathrm{mAb} 3 \mathrm{~B} 5$ \\
\hline $\operatorname{tg}($ homo $)$ PrP SH-SY5Y & $1.14 \times 10^{4}$ & $2.23 \times 10^{4}$ & $4.17 \times 10^{4}$ \\
$\operatorname{tg}($ mafa $)$ PrP SH-SY5Y & $1.57 \times 10^{4}$ & $4.61 \times 10^{4}$ & $4.53 \times 10^{4}$ \\
$\operatorname{tg}($ sai $)$ PrP SH-SY5Y & $2.87 \times 10^{4}$ & $5.24 \times 10^{4}$ & $4.79 \times 10^{4}$ \\
$\operatorname{tg}($ wtd $)$ PrP SH-SY5Y & $1.28 \times 10^{3}$ & $5.19 \times 10^{3}$ & $2.05 \times 10^{3}$ \\
EMC SH-SY5Y & $1.27 \times 10^{3}$ & $6.90 \times 10^{3}$ & $1.36 \times 10^{3}$ \\
\hline
\end{tabular}

\subsubsection{Summary of transgenic PrP cell line characterisation}

To examine the species barrier for TSE transmission in vitro, transgenic cell lines were generated by retroviral transduction. This technique facilitates transgene insertion into the target cell genome and subsequent expression. The insertion was validated for H. sapiens, M. fascicularis, S. sciureus and O. virginianus PrP transgenes. All transgenes were integrated to comparable numbers into the genomes of HpL3-4 and SH-SY5Y target cell lines. For all species except O. virginianus, transgenic PrP mRNA and protein expression was high and on comparable levels. Similar amounts of PrP were demonstrated on human and macaque PrP expressing cells. The highest PrP levels were demonstrated with all mAb used on cells expressing squirrel monkey PrP. This is in line with the RNA and PrP expression data. All cell lines showed two distinct populations with low and high PrP surface abundance. This finding was confirmed with three different anit-prion mAb all binding to distinct PrP epitopes. Since surface bound PrP is a prerequisite for prion infection in cell culture(McNally, Ward, and Priola, 2009), all cell lines generated could be used for infection experiments to estimate in vitro species barrier for prion-infectious agents. 


\subsection{Infection of transgenic cell lines with prion-infectious agents}

\subsubsection{Detection of cell culture-adapted 22L Scrapie PrPres in permanently infected $\operatorname{tg}(\mathrm{mo}) \operatorname{PrP} \mathrm{HpL3}-4$ cells}

Detection of infectious prion protein $\mathrm{PrPSc}$ is based on its partial proteinase K (PK) resistance (McKinley, Bolton, and Prusiner, 1983). PrPres, the in vitro generated isoform of PrPSc, is also PK resistant but less stable (Neary et al., 1991). 22L is a cell culture adapted, mouse-passaged sheep scrapie strain (Maas et al., 2007) extensively used to model prion infection in mice (Kim et al., 1990b; Pan et al., 2005) and cell culture (Atarashi et al., 2006; Baron et al., 2006; McNally, Ward, and Priola, 2009; Milhavet et al., 2006; Nishida et al., 2000; Vorberg, Raines, and Priola, 2004). The $\operatorname{tg}(\mathrm{mo}) \mathrm{HpL3}-4$ cell line (kindly provided by Prof. Ina Vorberg, DZNE, Bonn) is known to permanently propagate Scrapie 22L PrPres and thus is very useful to establish suitable PK concentrations later applied in infection experiments.

$6 \times 10^{6}$ permanently 22L infected $\operatorname{tg}(\mathrm{mo}) \operatorname{PrP} \mathrm{HpL3}-4$ cells and non-infected cells, respectively, were harvested by scraping and processed by ultracentrifugation (2.2.3.2). The pellet was resolved in PBS and digested with 0, 2.5, 5, 10, 20 and $30 \mu \mathrm{g} / \mathrm{ml}$ PK. The samples were analysed on Western blots and PrPres was detected with mAb ICSM-18. To decrease prion infectivity (Manuelidis, 1997) and expose epitopes of the prion protein the blotting membrane was incubated in $4 \mathrm{M}$ guanidine thiocyanate. The results are shown in figure 3.17. $\mathrm{PrP}^{\mathrm{c}}$ was completely degraded in non-infected cells, when treated with PK concentrations higher than $2.5 \mu \mathrm{g} / \mathrm{ml}$ (data not shown). In permanently 22Linfected cells prion protein was detectable in the range of $2.5-20 \mu \mathrm{g} / \mathrm{ml}$. Appearance of PrPres could be followed by the detection of a band at $\sim 16 \mathrm{kDa}$ (figure 3.17). A complete shift to the lower molecular weight of PK-digested PrPres was seen in the $20 \mathrm{~g} / \mathrm{ml} \mathrm{PK}$ samples. To ensure the complete digest of $\mathrm{PrP}^{\mathrm{c}}$ in non-infected cells without removing less stable PrPres in infected cells, the following experiments were accomplished with $10 \mu \mathrm{g} / \mathrm{ml}$ PK. For infection experiments with low PrPres propagation $1 \mu \mathrm{g} / \mathrm{ml}$ PK was used. 


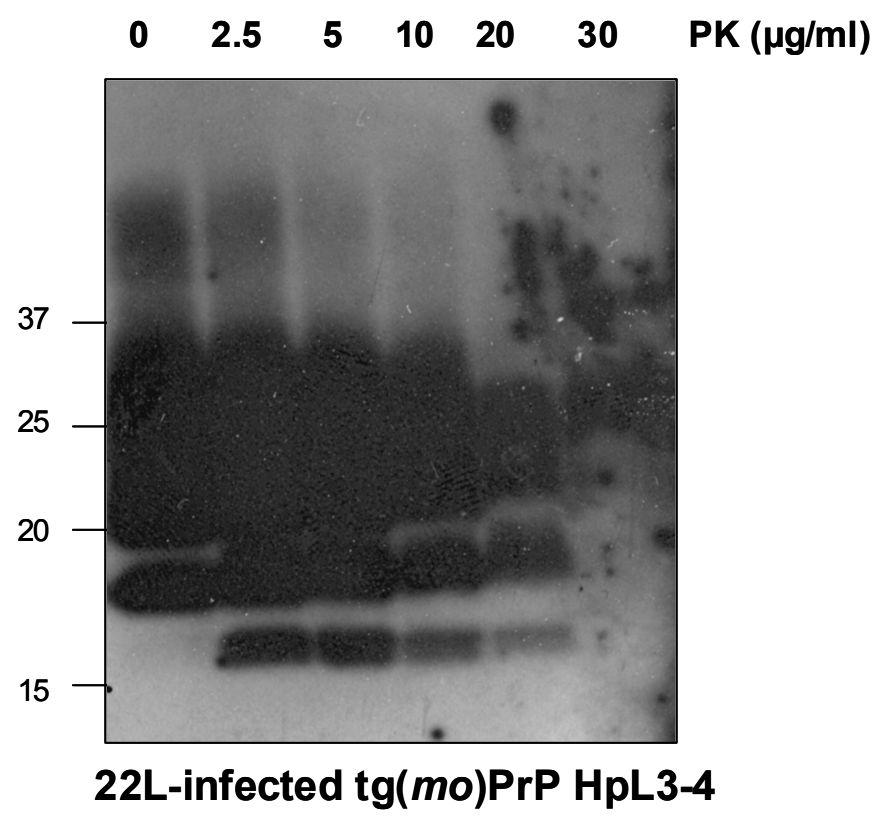

Figure 3.17: Detection of PrPres in 22L infected tg(mo)PrP HpL3-4 cells

Permanently 22L infected $\operatorname{tg}(\mathrm{mo}) \mathrm{PrP} \mathrm{HpL3}-4$ cells were harvested, processed by ultracentrifugation and digested with increasing amounts of proteinase K (PK). The samples were applied to Western blotting and $\mathrm{PrPc}^{\mathrm{c}}$ and $\mathrm{PrPres}$ was detected by $\mathrm{mAb}$ ICSM18. Corresponding molecular mass [kDa] are shown on the left.

\subsubsection{Influence of fetal calf serum on PrPres propagation in $\operatorname{tg}(m o) \operatorname{PrP} \mathrm{HpL3}$ - 4 cells}

An influence of different batches of fetal calf serum (FCS) on the stability of PrPres infection status in permissive cells was reported from different research groups (Profs. H.M. Schätzl, University of Wyoming, USA and I. Vorberg, DZNE, Bonn, personal communication). Thus, a failure to detect PrPres during cell culture infection could also be associated with the use of an inappropriate batch of FCS.

To minimise this effect on later infections of transgenic cells with various TSE inoculates, 8 different FCS batches were tested against a reference batch (Ref) known not to influence the propagation of PrPres in infected cells (kindly provided by Prof. I. Vorberg, Bonn). Tg(mo)PrP HpL3-4 and 22L permanently infected tg(mo)PrP HpL3-4 cells were seeded in 6-well plates and kept in cell culture medium supplemented with $10 \%$ FCS derived from different batches of the same supplier. Cells were cultured with continuous passaging and harvested at day 9. The cell lysates were processed as described (see above). Ultracentrifuge pellets were digested with $10 \mu \mathrm{g} / \mathrm{ml}$ Proteinase $\mathrm{K}$ (PK), according to the established protocol (see above). After Western blotting PrPres was detected with ICSM-18 (figure 3.18). 

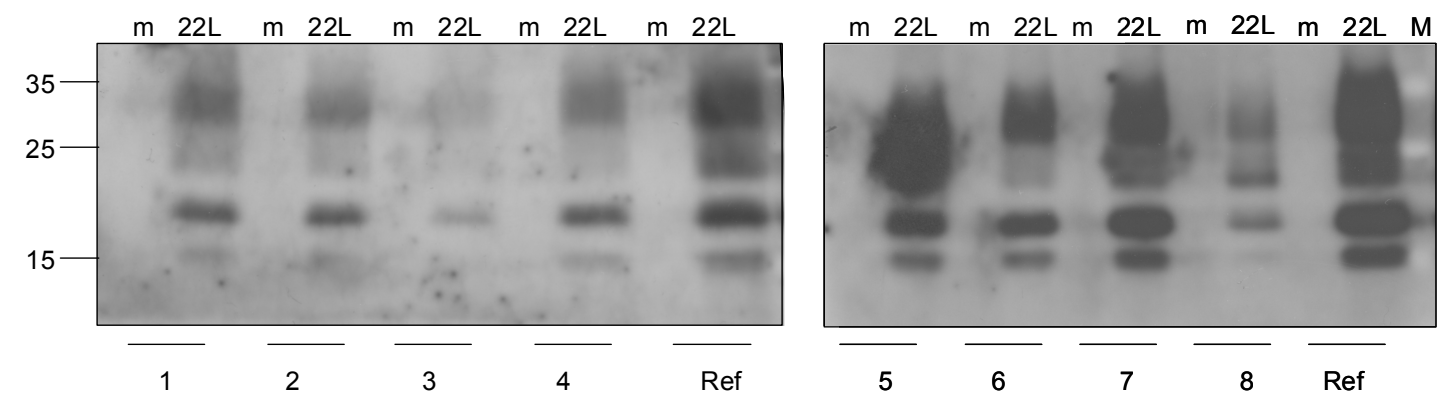

Figure 3.18: Influence of fetal calf serum on PrPres propagation in 22L infected $\operatorname{tg}(\mathrm{mo})$ PrP HpL3-4 cells

Permanently 22L infected $\operatorname{tg}(\mathrm{mo}) \mathrm{PrP} \mathrm{HpL} 3-4$ and non-infected $(\mathrm{m})$ cells were passaged for $9 \mathrm{~d}$ in media supplemented with different batches of FCS (1-8) or reference FCS (Ref). Lysates were processed by ultracentrifugation, digested with PK $(10 \mu \mathrm{g} / \mathrm{ml})$ and applied to Western blot analysis. PrPres was detected with mAb ICSM-18. The molecular mass $[\mathrm{kDa}]$ is indicated on the left.

As shown in figure 3.18, PK resistant PrP was not detected in all corresponding noninfected cell lysates. In all 22L infected samples PrPres was detectable, but in varying amounts. Use of FCS-3 showed the highest decrease in PrPres deposition (figure 3.17) in comparison to the reference FCS batch. Lower amounts of PrPres were also detected with FCS-1,-2,-4, -6 and -8. PrPres deposition was also not decreased for FCS-5 and 7. For the following infection experiments FCS-7 or the already tested reference FCS was used.

\subsubsection{Infection conditions for cell culture adapted 22L Scrapie in $\operatorname{tg}(m o) \operatorname{PrP}$ HpL3-4}

To test the susceptibility of and propagation of PrPres in the generated transgenic cell lines, different infection conditions were examined (adapted from H.M. Schätzl, personal communication) (Bian et al., 2010; Maas et al., 2007). Tg(mo)PrP HpL3-4 and EMC HpL3-4 cells were seeded in 6-well plates in duplicates. Cultures were inoculated on the following day with cell lysates derived from permanently Scrapie 22L-infected $\operatorname{tg}(m o)$ PrP HpL3-4 cells (which will be referred to as "cell culture adapted 22L"). The 22L inoculated EMC cells were carried along to monitor how long residual inoculum could be detected. The cell monolayers were incubated for $1 \mathrm{~d}$ with infecting lysate and then washed off. The cells were passaged at high confluency and lysate samples were obtained at 10, 13 and $18 \mathrm{dpi}$. After ultracentrifugation and PK digest with $1 \mu \mathrm{g} / \mathrm{ml}$, 
samples were applied to Western blot analysis, and PrPres was detected with mAb ICSM18.

Figure 3.18 shows the time course of an infection of $\operatorname{tg}(m o) \operatorname{PrP} \mathrm{HpL} 3-4$ cells with cell culture adapted 22L. Lane 1 shows PrPres in cell lysates of permanently infected cells after digestion with $10 \mu \mathrm{g} / \mathrm{ml}$ PK. This cell lysate corresponds to the amount of cell lysate used for the inoculation of $\operatorname{tg}(\mathrm{mo}) \operatorname{PrP} \mathrm{HpL3}-4$ cells. The signal from 22L inoculum further corresponds to the PrPres signals obtained after inoculation indicating a specific PrPres signal related to infection. This is further by absence of PrPres in the inoculated EMC control (cells, lane 8, 9). The $\operatorname{tg}(m o)$ PrP HpL3-4 cells (lane $2 \& 3$ ) showed PrPres with at $10 \mathrm{dpi}$. The $22 \mathrm{~L}$ was further propagated and stably detectable after 13 (lane 4, 5) and 18 dpi (lane 6, 7). At 18 dpi a strong increase of PrPres signal was detectable. Since after passaging 22L inoculated cells for $18 \mathrm{dpi}$ without loosing the PK resistant PrPres, cells were designated as permanently infected with the cell culture adapted Scrapie 22L strain. These established conditions were then used for all subsequent cell culture inoculation studies.

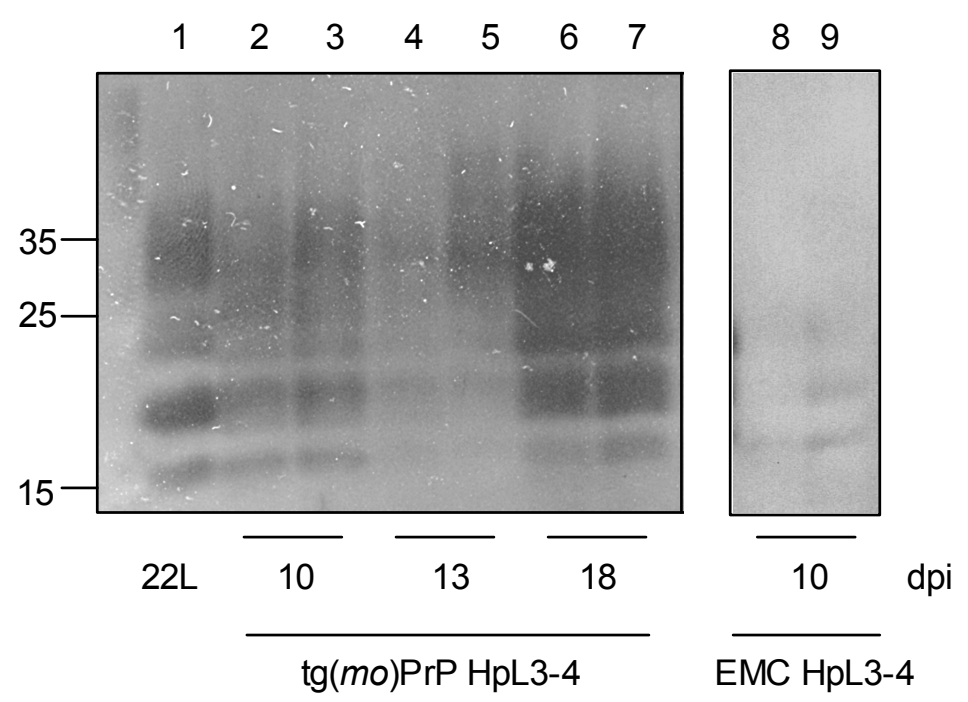

Figure 3.18: Infection of $\operatorname{tg}(\mathrm{mo}) \operatorname{PrP} \mathrm{HpL3}-4$ cells with cell culture adapted $22 \mathrm{~L}$

Two independent wells $\operatorname{tg}(\mathrm{mo}) \mathrm{PrP} \mathrm{HpL3}-4$ cells were inoculated with cell culture adapted 22L (lane 1), continuously passaged and expanded at dpi 10, 13 and 18. In parallel, EMC HpL3-4 cells (lane 8, 9) were also inoculated with cell culture adapted 22L and passaged for $10 \mathrm{~d}$. Cell lysates were processed by ultracentrifugation, PK digested and analysed by Western blot. PrPres was detected using mAb ICSM-18. The marker at the left indicates the molecular mass in $\mathrm{kDa}$. Duplicates correspond to independent infection of cells passaged and expanded in parallel for 18 days. 


\subsubsection{Inoculation of transgenic PrP expressing HpL3-4 and SH-SY5Y cell lines with cell culture adapted 22L}

To examine the interspecies transmission cell culture adapted $22 \mathrm{~L}$, the newly generated transgenic PrP HpL3-4 cells were inoculated (see above). Due to the PrP knock-out background of the murine host cell line (Kuwahara et al., 1999) endogenous mouse PrP could not interfere with the analysis of different PrP from several species.

Transgenic (homo)PrP-, (mafa)PrP-, (sai)PrP-, (wtd) PrP, (mo)PrP and EMC HpL3-4 cells were inoculated with cell culture adapted 22L in duplicates and passaged continuously. Samples were prepared at 10,17 and 24 and 43 dpi (corresponds to passage 3, 6, 9 and 17). Cells were harvested, processed by ultracentrifugation, PK digested $(1 \mu \mathrm{g} / \mathrm{ml})$ and analysed by Western blotting. As a positive control, cell lysates derived from permanently 22L infected cells were processed similarly except from the PK digest with $10 \mu \mathrm{g} / \mathrm{ml}$. PK-resistant PrP, indicating successful infection, was detected by mAb ICSM18.

In one of two replicates of 22L infected tg(homo)PrP HpL3-4 cells PrPres was detectable at $10 \mathrm{dpi}$ indicating a transient 22L infection of human PrP expressing cells (figure 3.19A). In comparison, transgenic (mafa)PrP and (wtd)PrP as well as EMC HpL3-4 cells did not contain PK resistant PrPres at all analysed time points. Obviously these cell lines were not susceptible to transient infection with the cell culture adapted 22L agent. In case of $\operatorname{tg}(w t d)$ PrP cells this may be caused by the low expression of PrP or the markedly reduced presentation of PrPres on the surface, as shown by Western blot (2.2.3.5) and flow cytometry (2.2.3.6). $\mathrm{Tg}(\mathrm{mo}) \mathrm{PrP} \mathrm{HpL} 3-4$ as a positive control showed PrPres in slightly variable amounts at all time points. Strikingly high amounts of PrPres were detected in both duplicates of $\operatorname{tg}($ sai $)$ PrP HpL3-4 cells at all time points with a strong increase from 10 to $17 \mathrm{dpi}$. This indicates a stable and permanent 22L infection of $\operatorname{tg}($ sai)PrP expressing HpL3-4 cells.

Take together, the inoculation with 22L revealed that $\operatorname{tg}($ sai $) \operatorname{PrP}$ expressing HpL3-4 cells, but not $\operatorname{tg}($ homo $) \operatorname{PrP}, \operatorname{tg}(m a f a) \operatorname{PrP}$ and $\operatorname{tg}(w t d) \operatorname{PrP}$ HpL3-4 cell lines, were susceptible to cell-culture adapted 22L and stably propagated PrPres. This partially reflects the in vivo situation as Scrapie was transmissible to squirrel monkeys (Gibbs et al., 1980). The propagation of cell culture adapted 22L in transgenic squirrel monkey PrP expressing cells was not shown before. 

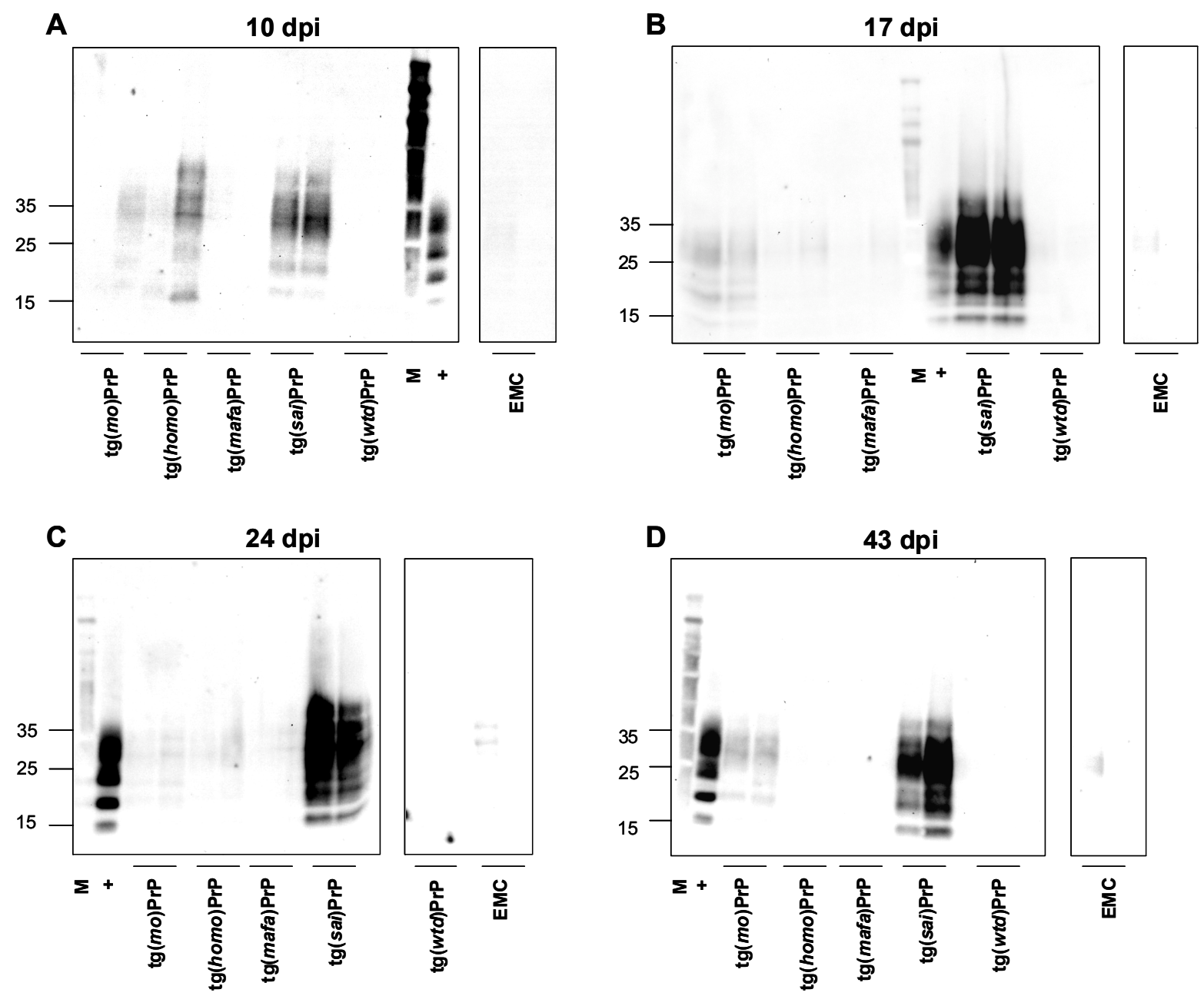

Figure 3.19: Infection of tg PrP HpL3-4 cells with cell culture adapted 22L

Transgenic HpL3-4 cells, expressing PrP from different species (M. musculus, H. sapiens, M. fascicularis, $S$. sciureus, 0 . virginianus) or EMC were inoculated in duplicates with cell culture adapted 22L lysates and passaged continuously. Cells from 10 (A), 17 (B), 24 (C) or 43 (D) dpi were expanded, harvested and processed by ultracentrifugation and PK digest. 22L infected $\operatorname{tg}(\mathrm{mo}) \mathrm{PrP}$ HpL3-4 cells (+) were treated with $10 \mu \mathrm{g} / \mathrm{ml} \mathrm{PK}$ and the 22L inoculated tg cell lines with $1 \mu \mathrm{g} / \mathrm{ml} \mathrm{PK}$. Samples were analysed by Western blotting and PrPres was detected with mAb ICSM-18. The molecular weight marker (M) shows the molecular mass in $\mathrm{kDa}$ (on the left of each blot). Note that duplicates correspond to independent infection of cells that were passaged and expanded in parallel for 18 days.

We then examined whether the donor species of the cell line influences the transmissibility of a prion-infectious agent. To date, the cell culture assays for prion diseases are usually performed on murine or rabbit host background, such as murine N2a, SMB, HpL3-4, GT-1 or rabbit RK13, or Rov cells (Vilette, 2008). With these cell lines, many co-interactors of the prion protein such as nucleic acids (Deleault, Lucassen, and Supattapone, 2003; Silva et al., 2010), receptors (Rieger et al., 1997), chaperones 
(Edenhofer et al., 1996) or others (Mays and Ryou, 2011) have been identified. One or more of those species-specific co-factors may be necessary for interspecies prion propagation. To analyse potential human susceptibility in cell culture, a humanized assay system is important. We inoculated the transgenic human SH-SY5Y cells according to the established protocol. To improve comparability the identical inoculum (Scrapie 22L lysate from permanently infected $\operatorname{tg}(\mathrm{mo}) \operatorname{PrP} \mathrm{HpL3}-4$ cells $)$ and a mock cell lysate derived from non-infected cells $\operatorname{tg}(m o) \operatorname{PrP}$ HpL3-4 cells was used. Tg(sai)PrP SH-SY5Y, and EMC SH-SY5Y were inoculated with the cell lysate in independent duplicates and passaged continuously. Additionally, $\operatorname{tg}($ sai)PrP SH-SY5Y cells were inoculated with mock lysates and carried along as a negative control to exclude that PrPres occurs spontaneously due to the high ectopic expression of the transgenes. Samples were continuously passaged and expanded at 8, 15, 20 and $40 \mathrm{dpi}$, harvested, processed by ultracentrifugation and PK digested. PrPres was detected by Western blotting with the mAb ICSM-18.

Tg(sai)PrP expressing SH-SY5Y cells did not support PrPres propagation (figure 3.20). Since we used inoculum that previously infected tg HpL3-4 cells we could exclude, that the inoculum was non-infectious. The conversion of $\operatorname{tg}($ sai $)$ PrP SH-SY5Y by cell culture adapted 22L was not successful, although the same transgene, expressed in a different host cell line (HpL3-4), resulted in conversion from $\mathrm{PrP}^{\mathrm{c}}$ to $\mathrm{PrPres}^{\mathrm{r}}$. The murine cell line may provide a cellular factor necessary for $22 \mathrm{~L}$ propagation. 

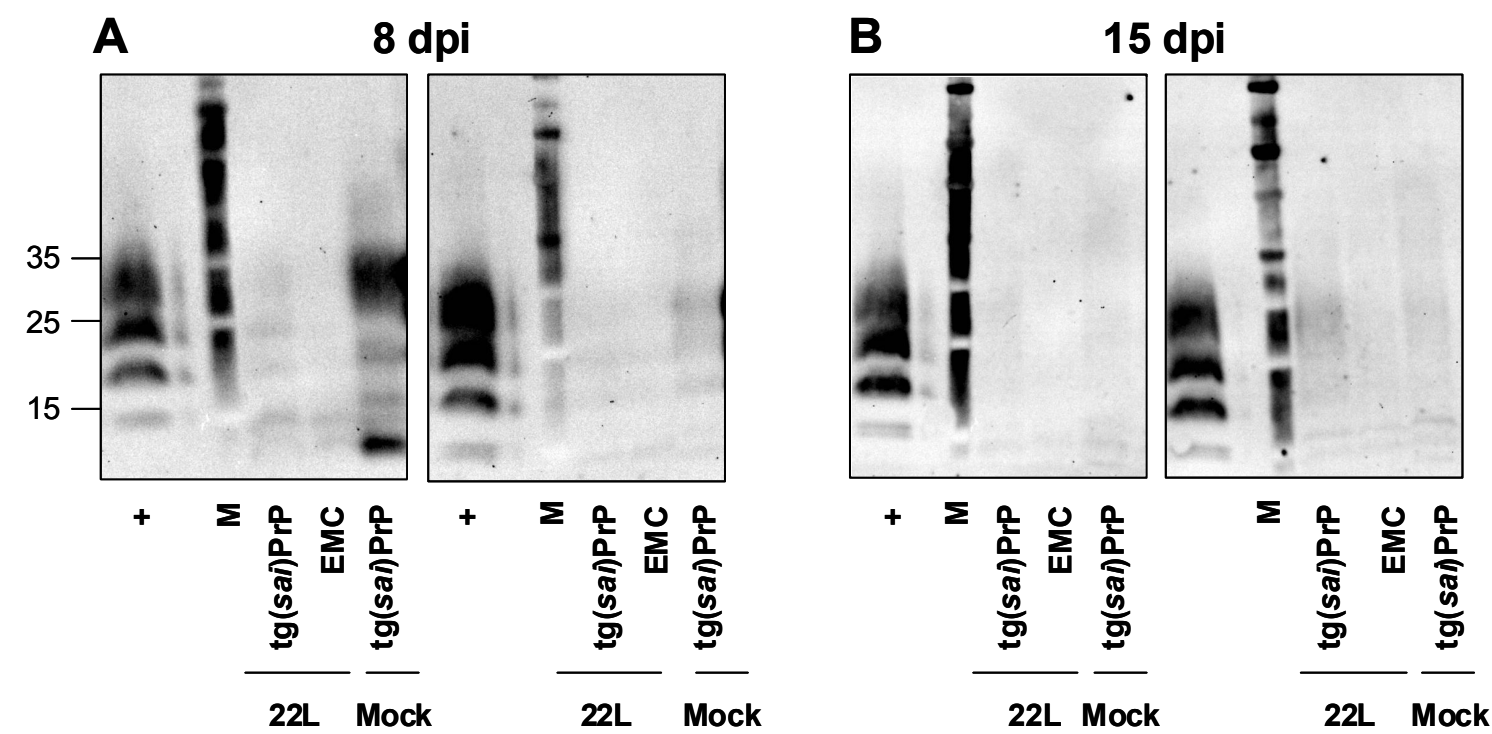

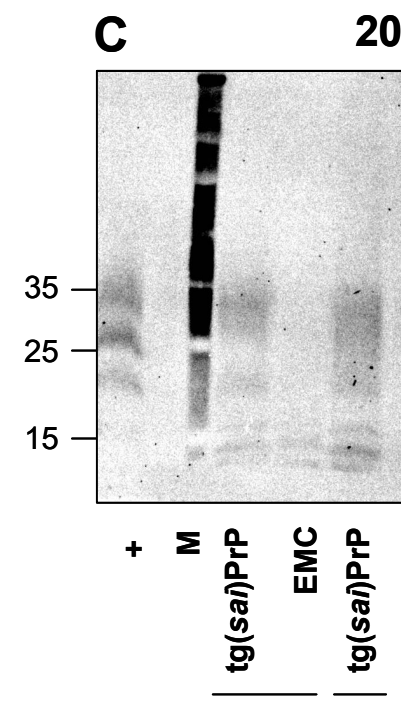

22L Mock
$20 \mathrm{dpi}$

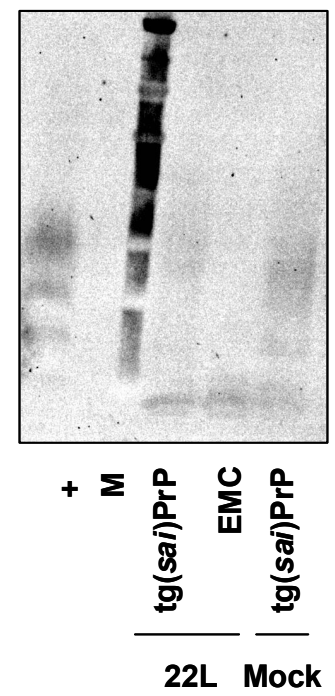

D

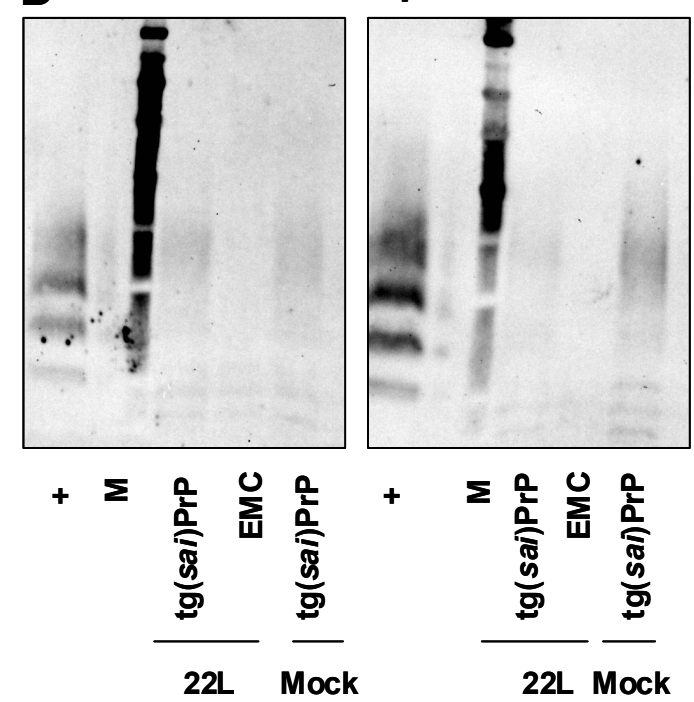

Figure 3.20: Inoculation of $\operatorname{tg}(s a i)$ PrP SH-SY5Y cells with cell culture adapted 22L Transgenic SH-SY5Y cells, expressing PrP from squirrel monkeys or EMC SH-SY5Y, were inoculated with cell culture adapted 22L lysate in duplicates and passaged continuously. Cells from 8 (A), 15 (B), 20 (C) and 40 (D) dpi were expanded, harvested and processed by ultracentrifugation and were PK digested. 22L infected $\operatorname{tg}(\mathrm{mo}) \mathrm{PrP}$ HpL3-4 cells $\mathrm{C}^{+}$ were treated with $10 \mu \mathrm{g} / \mathrm{ml} \mathrm{PK}$ and the 22L inoculated tg cell lines with $1 \mu \mathrm{g} / \mathrm{ml} \mathrm{PK}$. Samples were analysed by Western blot analysis and PrPres was detected with $\mathrm{mAb}$ ICSM-18. The molecular weight marker (M) shows the molecular mass in $\mathrm{kDa}$ (on the left of each blot). Note that duplicates correspond to independent infection of cells that were passaged and expanded in parallel for 18 days. 


\subsubsection{Inoculation of transgenic PrP expressing HpL3-4 cell lines with CWD}

To examine the interspecies transmission of primary Chronic wasting disease inoculum, the generated transgenic PrP expressing HpL3-4 cell line conversion to PrPres and subsequent propagation was analysed. A CWD transmission to humans, resulting in an acquired human prion disease such as VCJD so far has not been reported. Experimental CWD interspecies transmission to non-human primates had been accomplished. The preliminary results obtained in cynomolgus macaques did not show progression to prion-related disease so far (Race et al., 2009). The squirrel monkey has already been shown to be susceptible for CWD (Marsh et al., 2005; Race et al., 2009). Thus we hypothesised that $\operatorname{tg}(s a i)$ cells may be able to propagate CWD in cell culture. As described above, $\operatorname{tg}(s a i) \operatorname{PrP}$ HpL3-4 cells were permissive for cell-culture adapted 22L, but a susceptibility of HpL3-4 cells to primary prion-infectious agents was not reported so far. In order to test the interspecies transmission of CWD in vitro, $\operatorname{tg}($ homo $) \operatorname{PrP}$, $\operatorname{tg}(m a f a) \operatorname{PrP}$ and $\operatorname{tg}(s a i) \operatorname{PrP}$ HpL3-4 cell lines were inoculated with brain homogenate derived from CWD-infected and non-infected white-tailed deer (kindly provided by Prof. Jürgen Richt, (Hamir et al., 2008)). The cells were passaged and expanded for $30 \mathrm{dpi}$. Cell samples were processed by ultracentrifugation, PK digested and analysed by Western blot. PrPres was probed with mAb ICSM-18.

Our analysis revealed that PrPres was not detectable at 30 dpi (data not shown) in $\operatorname{tg}($ homo $)$ PrP HpL3-4 cells as well as in $\operatorname{tg}($ mafa $)$ PrP HpL3-4. Obviously, CWD infectious agent did not induce conversion of human or macaque $\mathrm{PrP}^{\mathrm{c}}$ to $\mathrm{PrP}^{\mathrm{res}}$ in this in vitro assay.

Surprisingly, infection of tg(sai) PrP expressing HpL3-4 cells with CWD was detectable in two independent, parallel infected replicates after 30 days (figure 3.21). The molecular weight of PrPres glycoforms was similar to that in 22L cell lysate. Thus, PrPres in $\operatorname{tg}(s a i) \operatorname{PrP}$ is due to the conversion of $\operatorname{PrPc}^{\mathrm{P}}$ to PrPres induced by CWD. EMC controls did not signal residual inoculum. However, one out of two parallel replicates of the mock inoculated $\operatorname{tg}(s a i) \operatorname{PrP}$ HpL3-4 cells showed a strong signal (compare figure $3.21 \mathrm{~A}$ with fig B). This was not caused by an incomplete PK digest (data not shown). A contamination during cell inoculation, passaging or harvesting of lysates cannot be excluded. A spontaneous conversion of transgenic (sai) PrP due to high ectopic 
expression of the transgene may also have occurred. However, spontaneous PrPres was not seen in other experiments.

Inoculation with primary CWD inoculum revealed that $\operatorname{tg}($ sai $) \operatorname{PrP}$ expressing HpL3-4 cells, but not $\operatorname{tg}($ homo $) \operatorname{PrP}, \operatorname{tg}(m a f a) \operatorname{PrP}$ and $\operatorname{tg}(w t d) \operatorname{PrP}$ HpL3-4 cell lines, were susceptible to primary CWD infectious agent and propagated PrPres. Although it was previously demonstrated that HpL3-4 cells can propagate PrPres after infection with mouse-passaged Scrapie (Maas et al., 2007), this is the first report on squirrel monkey PrP overexpressing HpL3-4 cells are permissive to infection with a primary prioninfectious agent, i.e. brain homogenate from CWD infected white-tailed deer. The results of this in vitro assay are supported by in vivo results demonstrating that CWD can infect squirrel monkeys (Marsh et al., 2005; Race et al., 2009).

\section{$30 \mathrm{dpi}$}

A

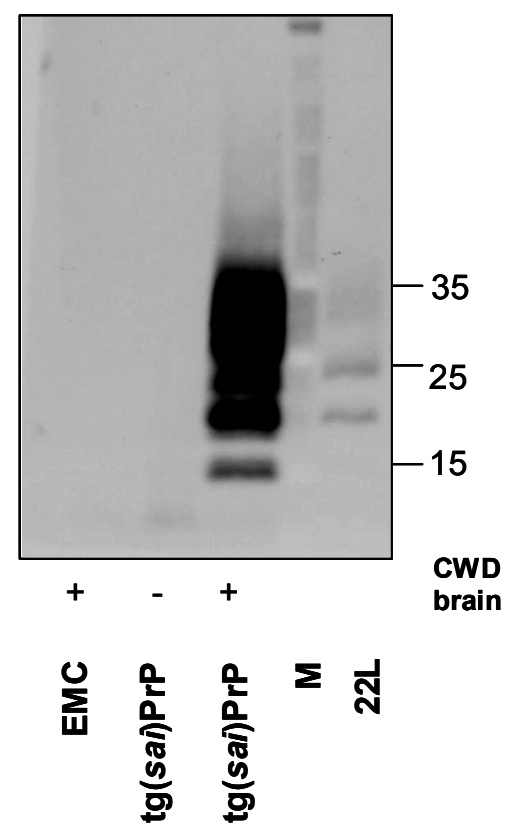

B

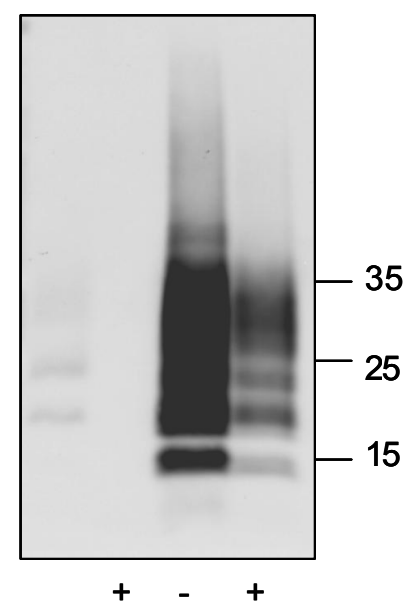

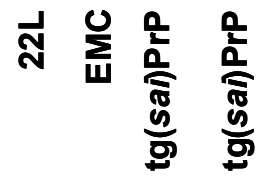

Figure 3.21: Infection of $\operatorname{tg}($ sai)PrP HpL3-4 cells with CWD brain

Tg(sai)PrP and EMC HpL3-4 cells were inoculated with CWD (+) or mock brain (-) homogenate in two parallel experiments (A, B), continuously passaged and expanded at $30 \mathrm{dpi}$. The cells were harvested, processed by ultracentrifugation, digested with PK and analysed by Western blot. PrPres was detected with mAb ICSM-18. 22L lysate was equally processed and applied as positive control (22L). The molecular mass in $\mathrm{kDa}$ is indicated on the right of each blot. Note, that duplicates correspond to independent infection of cells that were passaged and expanded in parallel for 18 days. Note the signal in mock-inoculated cells detected in one (B), but not in the other replicate (A). 


\subsection{Inoculation of cynomolgus macaques with CWD tissue using different inoculation routes}

In order to estimate the CWD transmission risk to humans, 18 cynomolgus macaques were inoculated with CWD infectious agents using different inoculation routes (table 2.15). The inoculation has started in May 2009; feeding of muscle tissue from CWD affected cervids is still ongoing. To date, all animals do not show prion disease-related clinical signs. Though, due to the long-lasting preclinical phase in prion diseases and experiences in prion transmission experiments to non-human primates (Drs. Dirk Motzkus, Edgar Holznagel, personal communication), clinical symptoms are so far not expected. 


\section{Discussion}

Chronic wasting disease is a neurodegenerative disease and classified as a transmissible spongiform encephalopathy occurring in four North American cervid species, Odocoileus virginianus, Odocoileus hemionus, Cervus elaphus nelsoni and Alces alces (Miller and Williams, 2004). It has recently emerged as a prion disease in captive as well as freeranging cervids most likely due to an apparent lack of biological and geographical barrieres. In the predominantly European roe deer species CWD has not been detected so far (De Bosschere et al., 2006; Roels et al., 2005; Schettler et al., 2006). However, a further, perhaps worldwide spread of CWD among cervids cannot be excluded. Despite culling of CWD harbouring farm animals the disease is on going. The rising incidence of CWD-positive free-ranging cervids remains an issue. The prevalence of CWD in endemic areas like Wyoming (USA) can reach up to $90 \%$ in captive herds and almost $50 \%$ in freeranging herds (Williams, 2005). The rapid spread via horizontal transmission and increase of prevalence in cervids within the last decade suggests that exposure of CWD to humans has already occurred in a large number of cases. Whether or not CWD, like BSE, is transmissible to humans cannot yet be answered with certainty. To date, surveillance data collected in the North American population did not hint to an increase of vCJD in CWD epidemic areas in North America. As we have learned from vCJD, which originated from cattle BSE, an animal prion disease can cross the species barrier to humans and other mammals by consumption of contaminated food. Hunting and meat processing practices suggest that consumed meat might have been in contact with highly infectious saliva, brain or lymphoid tissue. Moreover, PrPCWD was already detected in muscle tissue of infected deer (Angers et al., 2006). Game and hunt farms sheltered 54'000 cervids and accounted for \$60 million of the economy of Saskatchewan, Canada in 2010 (Douglas, 2011).

\subsection{CWD risk assessment using non-human primate in vivo and in vitro models}

In order to estimate the transmissibility of CWD to humans, a comprehensive CWD risk assessment study in cynomolgus macaques has been conducted at the German Primate 
Center, funded by the Alberta Prion Research Institute (Alberta, Canada) and the Ministry of Science and Culture (Lower Saxony, Germany).

To date, experimental transmission of CWD to cattle (Hamir et al., 2001) and transgenic mice (Browning et al., 2004) has been reported. Additionally, experimental transmission of CWD to four North American rodent species including meadow voles, red-backed voles, white-footed mice and deer mice was successful (Heisey et al., 2010). To test the zoonotic potential of CWD for humans it is necessary to use a species previously shown to mimic prion interspecies transmission of BSE to humans, such as the cynomolgus macaque (Lasmezas et al., 2005; Lasmezas et al., 1996). In addition, old world monkeys like cynomolgus macaques are evolutionary closer to humans (Hayasaka, Gojobori, and Horai, 1988). This close relationship between humans and cynomolgus macaque is also supported by the highly homologous amino acid sequence of PrP. Furthermore, except for one individual (Gibbs and Gajdusek, 1972), cynomolgus macaques are not susceptible to Scrapie and Scrapie infection is unknown in humans.

The zoonotic potential of CWD is evaluated by CWD transmission through different inoculation routes to groups of animals (table 2.15). To evaluate the putative CWDinfectivity of cervid-derived meat, muscle and brain tissue from diseased animals was used for oral transmission to cynomolgus macaques by multiple uptake events. Effective transmission of CWD to macaques was achieved by intracerebral transmission of brain homogenate from CWD-infective WTD and elk. Moreover, horizontal transmission was investigated by transfusion of blood from a sub-clinical CWD inoculated macaque.

Currently, the use of non-human primates for risk assessment studies of zoonotic prion diseases is the only experimentally feasible way to estimate the risk to CWD infection for humans. CWD transmission studies in non-human primates demonstrated susceptibility of the squirrel monkey (Marsh et al., 2005; Race et al., 2009), indicating that CWD can infect a non-human primate species with high attack and mortality rate. To date, CWD has not been transmitted to cynomolgus macaques (Race et al., 2009). However, due to the lack of sensitive intra vitam tests and an assumed long lasting preclinical incubation period for old world primates including humans CWD transmission to these species cannot yet be ruled out.

To shed some light on the potential in vivo transmission of CWD to primates including humans, $\operatorname{PrP}$ of H. sapiens, M. fascicularis, S. sciureus and O. viriginianus was cloned and overexpressed in suitable recipient cell lines for in vitro CWD transmission studies. To 
test the reliability of this cell culture assay, cell lines overexpressing PrP from CWDsusceptible white-tailed deer were generated as a positive control. The produced transgenic cell lines allow studies on prion infection in vitro and could be useful perhaps as a diagnostic tool in an ongoing study on CWD transmission to cynomolgus macaques in vivo.

\subsection{Modelling prion transmission in vitro}

A cell culture system to analyse the interspecies transmission of CWD was chosen. Cell cultures are versatile and provide a highly controlled system. Interfering factors such as genetic variance, reproducibility and implementation are assessed in a more reliable way compared to the analysis of a whole organism. The markedly reduced time from inoculation to PrPres accumulation, in conjunction with a high number of replicates, provides a powerful tool in a well controlled format with statistical reliability. Prion cell culture has two major challenges, the permissibility of cells to primary prion inocula and the stable propagation of prions. Only a few cell lines have been identified that provide these characteristics. To analyse the interspecies transmissibility of CWD, it is necessary to ensure that the endogenous protein expression does not interfere with the transgeneexpressed heterologous protein. An uncontrolled co-expression may lead to the inhibition of conversion or to false negative results, that has already been demonstrated in transgenic mouse models (Scott et al., 1993; Telling et al., 1995). To exclude interfering co-expression of endogenous PrP, the PrP ablated HpL3-4 cell line was chosen. This neuronal cell line was generated from the hippocampus epithelia of a $\mathrm{PrP} 0 / 0$ mouse (Kuwahara et al., 1999). The cell line is susceptible to and propagates mousepassaged Scrapie (Maas et al., 2007) when mouse PrP was ectopically expressed in these cells. This study demonstrated for the first time that prion infectivity could be rescued in PrP-deficient, resistant cell line by an ectopically expressed $\mathrm{PrPc}$.

The PrP-humanized mouse model is limited with respect to modelling the interspecies transmission of human prion diseases and unknown reasons for non-susceptibility (Bishop et al., 2006; Scott et al., 1993). Therefore, a human cell line with the characteristics of susceptible murine cell lines was also used for this study. The majority of permissive murine cell lines are derived from neuroblastomas with a fast cell division. 
They grow well in culture as the common murine neuroblastoma cell line N2a (Race, Fadness, and Chesebro, 1987).

These cell lines consistently propagate rodent-adapted Scrapie strains (Mahal et al., 2007) and have been used to analyse molecular mechanisms of prion conversion. Thus, the human neuroblastoma cell line SH-SY5Y was selected for work on this thesis. This cell line displays neuronal characteristics, e.g. neurite extension (Biedler, Helson, and Spengler, 1973), neurotransmitter synthesis (Biedler et al., 1978) and dopaminergic activity (Hillion et al., 2002). In dopaminergic regions of the brain, such as the substantia nigra, PrPSc deposition was detected (Hirose et al., 2006; Iwasaki et al., 2006; Vital et al., 2009). The loss of dopaminergic neurons in transgenic mouse models for neurodegeneration was demonstrated as well (Masliah et al., 2000). Furthermore, the SH-SY5Y cell line has been used in a prion overexpression study to analyse proteome expression changes (Weiss et al., 2010). Most importantly, this cell line was reported to be susceptible to sCJD prions in vitro (Ladogana et al., 1995).

\subsection{Overexpression of PrP in prion susceptible cell lines}

Prion infectivity in cell culture is dose dependent (Edgeworth et al., 2009; Klohn et al., 2003). Therefore it is necessary to provide these cells as transgenes $\mathrm{PrP}^{\mathrm{c}}$ for all species to be analysed for effective expression. Otherwise, the PrPres propagation cannot be linked to an interspecies transmission. Consequently, it was essential to produce cell lines that expressed comparable levels of transgenic $\mathrm{PrP}^{\mathrm{c}}$ independent of the transgene donor species. This is one pre-requisite for in vitro prion infection and propagation experiments. This condition allows discriminating between susceptibility and propagation of PrPres from the inoculum. The selection of donor species of PrP in trans has the advantage of an identical genetic background of the acceptor cell. Transduction is defined as virus-mediated gene delivery. Retroviral expression systems permanently integrate into the host cell genome and allow long-term observations (Varmus et al., 1977). For the generation of retroviral particles, gag, pol and env are necessary proteins. Viral particles with the VSV-G envelope protein were produced. Other commercially available systems often use the amphotropic envelope protein, 10A1 or an ecotropic envelope protein. However, these envelope proteins need defined cell surface receptor molecules for efficient transduction. These might be present on one, but not on another cell line. The VSV-G protein primarily recognises the phospholipid membrane present on 
all eukaryotic cells (Hall, Burson, and Huestis, 1998; Mastromarino et al., 1987) and as such can transduce different cell types of different host species. The use of retroviral vectors allows the transduction of a wide range of cells from different species together with the precise integration of genetic material and expression of transduced genes at high levels (Miller, 1997). The use of VSV-G revealed high efficient gene transfer into a variety of cells (Burns et al., 1993; Naldini et al., 1996; Yang et al., 1995). The transduction gene delivery system was combined with a retroviral expression system providing the integration of a transgene into the host cell genome.

To achieve highly comparable transgenic PrP expression among all cell lines, regulatory elements in the 5'- and the 3' UTR of the transgene should be eliminated. Such constructs solely amplify the ORF of the respective PrP cloned into the retroviral vector facilitating identical regulation for different transgenes. The relative transcription rate of the transgenic RNA only depends on the promoter activity of the vector construct provided by the flanking LTR regions. Integration of the retroviral transgene is undirected and requires the viral integrase and flanking LTRs. Integration does not prefer particular chromosomal loci. Thus, potential epigenetic effects on the transgenes are randomised leading to a comparable transgene RNA expression. The stability of retroviral integration and constant expression of transgenic PrP is beneficial for longterm infection experiments.

Transduction with viral GFP reporter particles was used to optimise the system. The final experimental set-up efficiently produced the transgene product in comparable amounts. Here, both neuronal cell lines originate from different species (mouse and human) but also from different tissues (hippocampus epithelium and neuroblastoma). Nonetheless, the optimised transduction conditions resulted in comparable levels of RNA expression in both cell lines confirming the suitability of the chosen conditions. Most likely transduction of other cell lines, e.g. lymphatic cells can be achieved with a similar outcome.

Probably the consistent RNA expression resulted from similar copy numbers of transgene integration events. The established transfection and transduction protocol combined with the antibiotic selection of positively transduced cells was highly effective and independent of the species and tissue origin of both the transgene donor and the acceptor host cell. 


\subsection{The amount of prion protein expression correlates with RNA expression data}

The propagation of prion-infectious agents strictly dependents on prion protein expression and cell surface display as $\mathrm{PrP}^{0 / 0}$ mice fail to develop prion disease after Scrapie inoculation(Bueler et al., 1993; Weissmann et al., 1994a; Weissmann et al., 1994b). Therefore, we assessed the prion protein expression in the produced cell lines by qPCR and Western blotting. In the EMC HpL3-4 and SH-SY5Y cells neither PrP-RNA expression nor endogenous prion protein was detectable. In transduced cell lines prion protein expression varied slightly among the different transgenes. This variation was presumably not caused by host specific regulation since promoter activity of flanking LTRs, identical in all cell lines, regulate transgene expression. Comparable prion protein expression levels were found in all HpL3-4 and SH-SY5Y cell lines, except $\operatorname{tg}(w t d) \mathrm{SH}-$ SY5Y. Here PrP expression was lower and reduced on the surface. But qPCR confirmed successful integration and robust transcription of cervid PrP. This effect was also not due to the vector-transgene construct. The ORF of cervid PrP was identical to the reference data base entries with functional start and stop codon proven by multiple sequencing.

Furthermore, this could be mediated by the choice of anti-prion antibody that may have resulted in different binding capacities to specific conformations of the prion protein (Martucci et al., 2009; Tang et al., 2010). However, the epitope recognised by the mAb ICSM-18 used for Western blotting is identical among all species like that of mAb 3B5 used in PrP surface detection. Moreover, for it is unlikely that the mAb 3B5 epitope is inaccessible. It binds to the flexible and less structured repetitive octapeptide region (Zahn et al., 2000) presenting multiple binding sites for the antibody. Since $\operatorname{tg}(w t d) \operatorname{PrP}$ RNA was present, but $\operatorname{tg}(w t d)$ prion protein was detected only faintly posttranscriptional mechanisms might be acting perhaps through miRNAs. MiRNA-342-3p is upregulated in CJD patients and BSE-infected macaques (Montag et al., 2009) and in Scrapie-infected mice the miRNA pattern changes (Saba et al., 2008) due to neuronal decay. MiRNA changes have also been observed in Alzheimer's disease (Provost, 2010). However, a cervid PrP regulating miRNA has so far not been described. The reason for the low expression of $\operatorname{tg}(w t d)$ PrP protein in HpL3-4 and SH-SY5Y cells remains unclear and warrants further investigations. 


\subsection{Flow cytometric quantification of PrP presenting HpL3-4 and SH- SY5Y cell lines}

Considering the random integration of PrP by retroviral transduction a heterogeneous population of PrP presenting cells can be expected. One cell clone may have numerous integration sites, some of them with higher transcriptional activity while others produce less. Therefore, we expected a heterogeneous expression and abundance of PrP on the cell surface as represented by a single population of cells with Gauss distribution of PrP on the cell surface. However, this was not observed. Most of the transgenic cell lines showed two distinct populations representing cells with low or high PrP abundance. Tg(sai) HpL3-4 high expressing cells have tenfold more surface PrP than the low expressing cells. Whether this distinct presentation of $\operatorname{PrP}$ on the surface of neurons is present in vivo is unknown. However prion expression varies among different brain regions among neuronal cell types (Beringue et al., 2003; Laine et al., 2001; Liu et al., 2001; Viegas et al., 2006) and also within the subcellular localisation, i.e. soma, dendrite or axon. The observed two cell populations with low or high PrP presentation may represent two different cell cycle stages (Gray et al., 1986; Rieseberg et al., 2001). Surface PrP may lower just after than prior to division. However, this would have resulted in a classical cell cycle pattern, which was not the case. Moreover, cultured cells tend to grow asynchronously and segmentation into two distinct cell populations seems unlikely.

Cell culture techniques can influence cell surface protein abundance. Harsh removal of cells from cell culture dishes decreases surface proteins (Canavan et al., 2005; Schmitt et al., 2005). To overcome this problem a large variety of mild detachment reagents are available for flow cytometry applications. As shown here the choice of detachment has a major influence on PrP abundance. Even a more gentle enzymatic detachment procedure dramatically decreases PrP surface concentration. Mechanical detachment left more PrP on the surface. Although single cell gating was achieved during the flow cytometry, the population with higher surface PrP may be explained by a cell clustering.

In conclusion, flow cytometric quantification of transgenic cell surface expression of PrP revealed an important new characteristic of the transgenic cell lines produced unassessible by mearly measuring RNA (qPCR) and/or total protein expression 
(Western blot). The abundance of $\mathrm{PrPc}^{\mathrm{c}}$ at the cell surface is a basic prerequisite for infection with a prion-infectious agent (Peretz et al., 2001; Priola and McNally, 2009).

After confirmation of $\mathrm{PrP}^{\mathrm{c}}$ expression and surface abundance, prion susceptible cells were inoculated with prion-infectious agents to analyse the species barrier in the $\mathrm{PrP}^{0 / 0}$ cell line HpL3-4 susceptible to mouse-passaged Scrapie by PrP overexpression (Maas et al., 2007). In this cell line the prion proteins from the species $H$. sapiens, $M$. fascicularis, $S$. sciureus and $O$. virginianus were overexpressed. The susceptibility to infection of the newly generated cell lines was probed by infection with 22L, a cell culture adapted mouse passaged Scrapie strain. An 22L lysate from permanently infected $\operatorname{tg}(\mathrm{mo}) \operatorname{PrP}$ HpL3-4 cells was applied (kindly provided by Dr. I. Vorberg). This cell culture adapted PrPres propagated permanently in the established cell lines.

Moreover assay parameters were optimised, such as FCS batch, cell density, and inoculum preparation, choice of time points for measuring PrPres and detection of PrPres by Western blot. Thus propagation of different prion-infectious agents should only depend on the transgene and the susceptibility of the cell line.

\subsection{The tg(sai)PrP HpL3-4 cell line is susceptible to cell culture adapted 22L}

Propagation of culture adapted 22L prions in $\operatorname{tg}($ homo $) \operatorname{PrP}, \operatorname{tg}($ mafa $) \operatorname{PrP}, \operatorname{tg}($ sai $) \operatorname{PrP}$ and $\operatorname{tg}(w t d)$ HpL3-4 cells was strongly species-dependent. Although experimental transmission of Scrapie to cervid species or PrP cervidized mice has been achieved (Green et al., 2008; Hamir et al., 2004a) our tg(wtd)PrP HpL3-4 cell line was not susceptible. Perhaps conversion of $\operatorname{tg}(w t d) \operatorname{PrP}$ to PrPres did not occur due to the low protein expression and the low cervid PrP surface abundance on this cell line critical for prion transmission (Bueler et al., 1993; Weissmann et al., 1994a).

Our inoculation experiments showed that cell-culture-adapted 22L did not stably infect $\operatorname{tg}($ homo $) \operatorname{PrP}$ and $\operatorname{tg}(\mathrm{mafa}) \operatorname{PrP}$ HpL3-4 cells. $\operatorname{tg}($ homo $) \operatorname{PrP}$ were only transiently infected in one replicate well at $10 \mathrm{dpi}$. Similarily, there have been no speculations that Scrapie can be transmitted to humans. However, experimental transmission of of Scrapie to a cynomolgus macaque has been published (Gibbs and Gajdusek, 1972) implying that transmission is possible although with a low attack rate and a long preclinical incubation time of more than 5 years. Within the same study, one rhesus macaque, one 
African green monkey and one chimpanzee was inoculated with the same inoculum and remained asymptomatic and so we cannot rule out that further passaging of cells might have revealed PrPres.

Interestingly, inoculation of $\operatorname{tg}($ sai $) \operatorname{PrP} \mathrm{HpL} 3-4$ cells with $22 \mathrm{~L}$ revealed that at all analysed time points PrPres was detectable. The squirrel monkey has previously been described as susceptible for Scrapie and CWD (Gibbs et al., 1980; Race et al., 2009). Taken together, this in vitro analysis showed high consistency to in vivo Scrapie transmission experiments.

\subsection{Susceptibility to prion infectious agents is cell line-dependent}

Both, $\operatorname{tg}($ sai $)$ PrP HpL3-4 and tg(sai)PrP SH-SY5Y express and present transgenic PrP of the squirrel monkey. To determine if susceptibility is transgene specific or influenced by the host cell line, we inoculated the $\operatorname{tg}($ sai $) \mathrm{PrP}$ SH-SY5Y. If only $\mathrm{PrPc}^{\mathrm{c}}$ abundance on the surface is necessary to induce conversion of $\operatorname{tg}($ sai $) \mathrm{PrP}^{c}$ to $\operatorname{tg}($ sai $) \mathrm{PrPres}$, each $\operatorname{tg}($ sai $) \mathrm{PrP}^{\mathrm{c}}$ overexpressing cell line should propagate PrPres after inoculation with prion-infectious agent; this was not the case. The tg(sai)PrP HpL3-4 cell line propagated PrPres from 22L cell culture adapted inoculum, but not $\operatorname{tg}($ sai) PrP SH-SY5Y cells. Our results indicate that the prion susceptibility and PrPres propagation is independent of prion protein overexpression and is more likely defined by the genetic background of the recipient cell line, because both, $\operatorname{tg}($ sai)PrP HpL3-4 and SH-SY5Y express and present squirrel monkey PrP on comparable levels. Therefore, the presentation of a functional $\mathrm{PrP}^{\mathrm{c}}$ on the cell surface cannot be the exclusive requirement for infection due to the fact that different cell lines, which comprise transgenic PrP abundance of the same species, are not equally susceptible. Moreover, none of the transgenic SHSY5Y was susceptible to any of the infectious reagents used.

In contrast, the susceptibility of wildtype SH-SY5Y cells to prion-infectious agent was reported on one occasion (Ladogana et al., 1995). The authors postulated that SH-SY5Y and subclones thereof could be persistently infected with human SCJD brain homogenate. Using a similar experimental setup PrPres propagation was not detected over a time period of $40 \mathrm{dpi}$, either with with mouse-adapted Scrape 22L cell lysate nor with primary CWD brain.

It was an exception that primary CJD agent did infect wild type SH-SY5Y. However, these results have not been confirmed so far (neither by the authors themselves or by other 
groups). Regarding the endogenous expression of PrPc, we did not detect traces of PrPRNA in SH-SY5Y. This is in conflict to the findings of Ladogana and colleagues, who showed CJD propagation in this human neuroblastoma cell line (Ladogana et al., 1995) indicating that the SH-SY5Y cell line expresses prion protein constitutively at high levels. However, the expression and quantification of PrP transcripts was not demonstrated within their study. We could show that in EMC SH-SY5Y PrP expression was not detectable, either by qPCR $(3.3 .1,3.3 .2)$, Western blot analysis (3.3.3) or flow cytometry (3.3.4) by the use of antibodies binding to different epitopes. The only reason for the susceptibility to primary inocula could be the multiple subcloning accomplished in the study of Lagodana and colleagues that could have led to PrPc expressing subpopulations, which gained susceptibility to prions.

\subsection{Tg(sai)PrP HpL3-4 cells are susceptible to infection with primary CWD inoculum}

To test transmissibility of CWD in vitro, it is necessary to generate a cell line, which is susceptible to primary inocula. Due to the lack of susceptibility of SH-SY5Y cell lines, the transgenic PrP expressing HpL3-4 cell lines were explored further. The susceptibility to mouse passaged Scrapie (Maas et al., 2007) and cell culture adapted 22L (3.3.4) was already demonstrated. Infection of cell cultures with mouse-adapted Scrapie strains is widely used to model the conversion of PrP in vitro. Furthermore, cell culture assays have been developed to discriminate and characterise mouse-adapted Scrapie strains. However, these assays are insufficient to mimic the species barrier of a particular prion disease due to their non-susceptibility to primary inocula. The inoculation with primary agents remains challenging. The only studies that demonstrate the susceptibility to a primary inoculum so far was transmission of sheep Scrapie to a rabbit kidney cell line (Vilette et al., 2001). The in vitro transmission of primary CWD inoculum has so far only been observed in primary mule deer neurons (Raymond et al., 2006). Additionally, the in vitro propagation in elk PrP overexpressing RK13 cells was only possible with mousepassaged CWD as primary inoculum (Bian et al., 2010). The authors concluded that the CWD infection was transient and only appeared between passage 3 to 5 . However, as shown in this thesis, at early time points residuals of inoculum are attached to the cells and can be detected by Western blot. False positive detection can only be ruled out using an adequate negative control, e.g. inoculation of EMC cells. However, this negative 
control was not mentioned by the authors (Bian et al., 2010) and is necessary to exclude false negative results, when analysing early time points in transient infections.

The most suitable species to test CWD infectivity in vitro as a proof of principle is a transgenic cell line expressing white-tailed deer PrP, because the horizontal transmission rate of CWD is highest in this species (Prof. Stefanie Czub, personal communication). In vivo transmission of CWD to hamster and PrP transgenic mice models has also been frequently demonstrated (Browning et al., 2004; Meade-White et al., 2007; Raymond et al., 2007; Seelig et al., 2010; Tamguney et al., 2006; Trifilo et al., 2007). To test the CWD interspecies transmission in vitro, we generated cervid $\operatorname{Pr} P$ overexpressing HpL3-4 cells. However, we could not detect susceptibility to primary CWD inoculum. This may be due to the low expression level of $\operatorname{tg}(w t d) \operatorname{PrP}$. The ultimate reason for the markedly lower expression of cervid PrP in these cells remains unclear and needs further investigation.

Furthermore, $\operatorname{tg}($ homo $)$ PrP HpL3-4 cells were tested for in vitro CWD susceptibility to model interspecies transmission from CWD to humans. The available experimental data do not suggest a zoonotic potential of CWD, but the transmission to humans due to the consumption of CWD contaminated food cannot be excluded. CWD transmission studies in PrP humanized mice revealed that these mice are not susceptible (Browning et al., 2004; Sandberg et al., 2010), the authors concluded that a substantial species barrier for transmission of CWD to humans must exist. Using the $\operatorname{tg}($ homo) PrP HpL3-4 cells we could confirm this result in our in vitro assay. The inoculation of $\operatorname{tg}($ homo)PrP HpL3-4 cells with primary CWD brain pool did not induce conversion and PrPres propagation was not detected.

However, the same humanized mouse models have been used to estimate the zoonotic potential of BSE (Asano et al., 2006; Kong et al., 2008) and these mice were not or only weakly susceptible to BSE, which does not reflect the in vivo transmissibility. The cynomolgus macaque is an accepted species to model human prion disease, the missing link between VCJD in humans and BSE in cattle was discovered by the inoculation of cynomolgus macaques with BSE-contaminated cattle products (Lasmezas et al., 2005). Cynomolgus macaques have already been inoculated with primary CWD isolates (Race et al., 2009) and as yet, no interspecies transmissibility was reported. Furthermore, estimating the species barrier of CWD in cynomolgus macaques by different inoculation routes has revealed no clinical symptoms to date, the inoculation started in May 2009. 
Whether or not CWD will induce prion disease in cynomolgus macaques cannot be predicted. After oral exposure with low doses of BSE, cynomolgus macaques remain asymptomatic for more than 4 years but the attack rate of the agent in six animals was 100\%. (D. Motzkus, personal communication). In humans the preclinical incubation periods can even last for decades (Collinge et al., 2008). The $\operatorname{tg}(\mathrm{mafa}$ )PrP HpL3-4 cells, which were used as a non-human primate in vitro model, showed that under the chosen conditions PrPres conversion was not induced.

However, non-susceptibility of the above mentioned species can only be assumed if the permissibility to primary CWD brain inoculum is confirmed for this cell line. Until now, the HpL3-4 cell line was only demonstrated for permissibility to mouse-passaged Scrapie. Surprisingly, HpL3-4 cells overexpressing PrP from the squirrel monkey, gained susceptibility to primary CWD inoculum. This in vitro transmission of primary CWD inoculum to a transgenic susceptible cell line was shown here for the first time. It should be emphasised that mock brain inoculated $\operatorname{tg}($ sai $) \operatorname{PrP}$ HpL3-4 cells did show PrPres in one out of two independently accomplished infections. If this is due to technical contaminations or spontaneous aggregation of $\mathrm{PrP}^{c}$ or due to $\mathrm{PrP}$ overexpression remains unclear. None of the other inoculation studies showed spontaneous PrPres formation in any of the produced transgenic cell lines (data not shown).

This is the first report that shows the susceptibility of a neuronal PrP rescued cell line to primary prion-infectious inoculum. This tg(sai) PrP cell line is susceptible to cell culture adapted mouse-passaged Scrapie and primary CWD inoculum. Interestingly, these transmission results fit to in vivo experimental data. The squirrel monkey was not only permissive for Scrapie infection (Gibbs et al., 1980), but also permissive to CWD infection (Marsh et al., 2005; Race et al., 2009). These new world monkeys showed prion-related clinical symptoms, characteristical spongiosis and $\mathrm{PrPSc}$ deposits. We found that after inoculation with primary CWD brain inoculum, $\operatorname{tg}($ sai) PrP HpL3-4 cells accumulate PrPres continuously. 


\section{Conclusion}

The use of cell culture in prion research is challenging; currently only a few susceptible cell lines are known to propagate infectivity of a primary inoculum. We established a transgenic cell culture assay to simulate CWD transmissibility in vitro. All of the produced transgenic cell lines were extensively characterised for PrP expression and surface abundance, and finally inoculated the cell lines with infectious agents derived from CWD-infected white-tailed deer. The outcome of this study showed CWD transmission only for the squirrel monkey PrP expressing cell line, which reflects the in vivo situation. The transmission to humanized cell lines could not be shown by this assay; however this does not exclude the potential risk to humans. In vitro assays can only assist the in vivo models to estimate the likelihood of transmission of CWD. There was a time, when BSE was also considered not to be risky to humans. 


\section{References}

Adjou, K. T., Simoneau, S., Sales, N., Lamoury, F., Dormont, D., Papy-Garcia, D., Barritault, D., Deslys, J. P., and Lasmezas, C. I. (2003). A novel generation of heparan sulfate mimetics for the treatment of prion diseases. J Gen Virol 84(Pt 9), 2595-603.

Aguzzi, A., Heikenwalder, M., and Polymenidou, M. (2007). Insights into prion strains and neurotoxicity. Nat Rev Mol Cell Biol 8(7), 552-61.

Aldhous, P. (1990). BSE: spongiform encephalopathy found in cat. Nature 345(6272), 194.

Alper, T., Haig, D. A., and Clarke, M. C. (1978). The scrapie agent: evidence against its dependence for replication on intrinsic nucleic acid. J Gen Virol 41(3), 503-16.

Angers, R. C., Browning, S. R., Seward, T. S., Sigurdson, C. J., Miller, M. W., Hoover, E. A., and Telling, G. C. (2006). Prions in skeletal muscles of deer with chronic wasting disease. Science 311(5764), 1117.

Angers, R. C., Kang, H. E., Napier, D., Browning, S., Seward, T., Mathiason, C., Balachandran, A., McKenzie, D., Castilla, J., Soto, C., Jewell, J., Graham, C., Hoover, E. A., and Telling, G. C. (2010). Prion strain mutation determined by prion protein conformational compatibility and primary structure. Science 328(5982), 1154-8.

Angers, R. C., Seward, T. S., Napier, D., Green, M., Hoover, E., Spraker, T., O'Rourke, K., Balachandran, A., and Telling, G. C. (2009). Chronic wasting disease prions in elk antler velvet. Emerg Infect Dis 15(5), 696-703.

Archer, F., Bachelin, C., Andreoletti, O., Besnard, N., Perrot, G., Langevin, C., Le Dur, A., Vilette, D., Baron-Van Evercooren, A., Vilotte, J. L., and Laude, H. (2004). Cultured peripheral neuroglial cells are highly permissive to sheep prion infection. J Virol 78(1), 482-90.

Asano, M., Mohri, S., Ironside, J. W., Ito, M., Tamaoki, N., and Kitamoto, T. (2006). vCJD prion acquires altered virulence through trans-species infection. Biochem Biophys Res Commun 342(1), 293-9.

Atarashi, R., Sim, V. L., Nishida, N., Caughey, B., and Katamine, S. (2006). Prion straindependent differences in conversion of mutant prion proteins in cell culture. $J$ Virol 80(16), 7854-62.

Aubin, R. A., Weinfeld, M., Taghavi, M., Mirzayans, R., and Paterson, M. C. (1997). Highly effective delivery of foreign DNA to adherent cells via polybrene/DMSO-assisted gene transfer. Methods Mol Biol 62, 319-42.

Baron, G. S., Magalhaes, A. C., Prado, M. A., and Caughey, B. (2006). Mouse-adapted scrapie infection of SN56 cells: greater efficiency with microsome-associated versus purified PrP-res. J Virol 80(5), 2106-17.

Basler, K., Oesch, B., Scott, M., Westaway, D., Walchli, M., Groth, D. F., McKinley, M. P., Prusiner, S. B., and Weissmann, C. (1986). Scrapie and cellular PrP isoforms are encoded by the same chromosomal gene. Cell 46(3), 417-28.

Belay, E. D., and Schonberger, L. B. (2002). Variant Creutzfeldt-Jakob disease and bovine spongiform encephalopathy. Clin Lab Med 22(4), 849-62, v-vi.

Ben-Zaken, O., Tzaban, S., Tal, Y., Horonchik, L., Esko, J. D., Vlodavsky, I., and Taraboulos, A. (2003). Cellular heparan sulfate participates in the metabolism of prions. J Biol Chem 278(41), 40041-9.

Benarroch, E. E., and Stotz-Potter, E. H. (1998). Dysautonomia in fatal familial insomnia as an indicator of the potential role of the thalamus in autonomic control. Brain Pathol 8(3), 527-30. 
Benestad, S. L., Sarradin, P., Thu, B., Schonheit, J., Tranulis, M. A., and Bratberg, B. (2003). Cases of scrapie with unusual features in Norway and designation of a new type, Nor98. Vet Rec 153(7), 202-8.

Beringue, V., Mallinson, G., Kaisar, M., Tayebi, M., Sattar, Z., Jackson, G., Anstee, D., Collinge, J., and Hawke, S. (2003). Regional heterogeneity of cellular prion protein isoforms in the mouse brain. Brain 126(Pt 9), 2065-73.

Bertani, G. (1951). Studies on lysogenesis. I. The mode of phage liberation by lysogenic Escherichia coli. J Bacteriol 62(3), 293-300.

Biacabe, A. G., Laplanche, J. L., Ryder, S., and Baron, T. (2004). Distinct molecular phenotypes in bovine prion diseases. EMBO Rep 5(1), 110-5.

Bian, J., Napier, D., Khaychuck, V., Angers, R., Graham, C., and Telling, G. (2010). Cellbased quantification of chronic wasting disease prions. J Virol 84(16), 8322-6.

Biedler, J. L., Helson, L., and Spengler, B. A. (1973). Morphology and growth, tumorigenicity, and cytogenetics of human neuroblastoma cells in continuous culture. Cancer Res 33(11), 2643-52.

Biedler, J. L., Roffler-Tarlov, S., Schachner, M., and Freedman, L. S. (1978). Multiple neurotransmitter synthesis by human neuroblastoma cell lines and clones. Cancer Res 38(11 Pt 1), 3751-7.

Birnboim, H. C. (1983). A rapid alkaline extraction method for the isolation of plasmid DNA. Methods Enzymol 100, 243-55.

Bishop, M. T., Hart, P., Aitchison, L., Baybutt, H. N., Plinston, C., Thomson, V., Tuzi, N. L., Head, M. W., Ironside, J. W., Will, R. G., and Manson, J. C. (2006). Predicting susceptibility and incubation time of human-to-human transmission of vCJD. Lancet Neurol 5(5), 393-8.

Bolton, D. C., McKinley, M. P., and Prusiner, S. B. (1982). Identification of a protein that purifies with the scrapie prion. Science 218(4579), 1309-11.

Borchelt, D. R., Taraboulos, A., and Prusiner, S. B. (1992). Evidence for synthesis of scrapie prion proteins in the endocytic pathway. J Biol Chem 267(23), 16188-99.

Bosque, P. J., and Prusiner, S. B. (2000). Cultured cell sublines highly susceptible to prion infection. J Virol 74(9), 4377-86.

Braun, U., Schicker, E., Pusterla, N., and Schonmann, M. (1998). [Clinical findings in 50 cows with bovine spongiform encephalopathy (BSE)]. Berl Munch Tierarztl Wochenschr 111(1), 27-32.

Brown, D. R., Besinger, A., Herms, J. W., and Kretzschmar, H. A. (1998a). Microglial expression of the prion protein. Neuroreport $\mathbf{9}(7), 1425-9$.

Brown, D. R., Qin, K., Herms, J. W., Madlung, A., Manson, J., Strome, R., Fraser, P. E., Kruck, T., von Bohlen, A., Schulz-Schaeffer, W., Giese, A., Westaway, D., and Kretzschmar, H. (1997). The cellular prion protein binds copper in vivo. Nature 390(6661), 684-7.

Brown, D. R., Schmidt, B., Groschup, M. H., and Kretzschmar, H. A. (1998b). Prion protein expression in muscle cells and toxicity of a prion protein fragment. Eur J Cell Biol 75(1), 29-37.

Brown, D. R., Schmidt, B., and Kretzschmar, H. A. (1996). Role of microglia and host prion protein in neurotoxicity of a prion protein fragment. Nature 380(6572), 345-7.

Brown, D. R., Schmidt, B., and Kretzschmar, H. A. (1997). Effects of oxidative stress on prion protein expression in PC12 cells. Int J Dev Neurosci 15(8), 961-72.

Brown, H. R., Goller, N. L., Rudelli, R. D., Merz, G. S., Wolfe, G. C., Wisniewski, H. M., and Robakis, N. K. (1990). The mRNA encoding the scrapie agent protein is present in a variety of non-neuronal cells. Acta Neuropathol 80(1), 1-6. 
Brown, P., Preece, M., Brandel, J.P., Sato, T., McShane, L., Zerr, I., Fletcher, A., Will, R.G., Pocchiari, M., Cashman, N.R., d'Aignaux, J.H., Cervenáková, L., Fradkin, J., Schonberger, L.B., Collins, S.J. (2000). Iatrogenic Creutzfeldt-Jakob disease at the millenium. Neurology 55, 1075-1081.

Browning, S. R., Mason, G. L., Seward, T., Green, M., Eliason, G. A., Mathiason, C., Miller, M. W., Williams, E. S., Hoover, E., and Telling, G. C. (2004). Transmission of prions from mule deer and elk with chronic wasting disease to transgenic mice expressing cervid PrP. J Virol 78(23), 13345-50.

Bruce, M., Chree, A., McConnell, I., Foster, J., Pearson, G., and Fraser, H. (1994). Transmission of bovine spongiform encephalopathy and scrapie to mice: strain variation and the species barrier. Philos Trans R Soc Lond B Biol Sci 343(1306), 405-11.

Bruce, M. E. (2003). TSE strain variation. Br Med Bull 66, 99-108.

Bryan, N., Andrews, K. D., Loughran, M. J., Rhodes, N. P., and Hunt, J. A. (2011). Elucidating the contribution of the elemental composition of fetal calf serum to antigenic expression of primary human umbilical-vein endothelial cells in vitro. Biosci Rep 31(3), 199-210.

Budka, H. (2007). Portrait of Creutzfeldt-Jakob Disease. Prions in Humans and Animals (editors: Hörnlimann B., Riesner D., Kretzschmar H.), 195-203.

Bueler, H., Aguzzi, A., Sailer, A., Greiner, R. A., Autenried, P., Aguet, M., and Weissmann, C. (1993). Mice devoid of PrP are resistant to scrapie. Cell 73(7), 1339-47.

Bueler, H., Fischer, M., Lang, Y., Bluethmann, H., Lipp, H. P., DeArmond, S. J., Prusiner, S. B., Aguet, M., and Weissmann, C. (1992). Normal development and behaviour of mice lacking the neuronal cell-surface PrP protein. Nature 356(6370), 577-82.

Burnette, W. N. (1981). "Western blotting": electrophoretic transfer of proteins from sodium dodecyl sulfate--polyacrylamide gels to unmodified nitrocellulose and radiographic detection with antibody and radioiodinated protein A. Anal Biochem 112(2), 195-203.

Burns, J. C., Friedmann, T., Driever, W., Burrascano, M., and Yee, J. K. (1993). Vesicular stomatitis virus $\mathrm{G}$ glycoprotein pseudotyped retroviral vectors: concentration to very high titer and efficient gene transfer into mammalian and nonmammalian cells. Proc Natl Acad Sci U S A 90(17), 8033-7.

Buschmann, A., Gretzschel, A., Biacabe, A. G., Schiebel, K., Corona, C., Hoffmann, C., Eiden, M., Baron, T., Casalone, C., and Groschup, M. H. (2006). Atypical BSE in Germany-proof of transmissibility and biochemical characterization. Vet Microbiol 117(24), 103-16.

Buschmann, A., Pfaff, E., Reifenberg, K., Muller, H. M., and Groschup, M. H. (2000). Detection of cattle-derived BSE prions using transgenic mice overexpressing bovine PrP(C). Arch Virol Suppl(16), 75-86.

Butler, D. A., Scott, M. R., Bockman, J. M., Borchelt, D. R., Taraboulos, A., Hsiao, K. K., Kingsbury, D. T., and Prusiner, S. B. (1988). Scrapie-infected murine neuroblastoma cells produce protease-resistant prion proteins. J Virol 62(5), 1558-64.

Calzolai, L., Lysek, D. A., Perez, D. R., Guntert, P., and Wuthrich, K. (2005). Prion protein NMR structures of chickens, turtles, and frogs. Proc Natl Acad Sci U S A 102(3), 651-5.

Canavan, H. E., Cheng, X., Graham, D. J., Ratner, B. D., and Castner, D. G. (2005). Cell sheet detachment affects the extracellular matrix: a surface science study comparing thermal liftoff, enzymatic, and mechanical methods. J Biomed Mater Res A 75(1), $1-13$. 
Carlson, G. A., Ebeling, C., Yang, S. L., Telling, G., Torchia, M., Groth, D., Westaway, D., DeArmond, S. J., and Prusiner, S. B. (1994). Prion isolate specified allotypic interactions between the cellular and scrapie prion proteins in congenic and transgenic mice. Proc Natl Acad Sci U S A 91(12), 5690-4.

Casalone, C., Zanusso, G., Acutis, P., Ferrari, S., Capucci, L., Tagliavini, F., Monaco, S., and Caramelli, M. (2004). Identification of a second bovine amyloidotic spongiform encephalopathy: molecular similarities with sporadic Creutzfeldt-Jakob disease. Proc Natl Acad Sci U S A 101(9), 3065-70.

Castilla, J., Gutierrez-Adan, A., Brun, A., Pintado, B., Parra, B., Ramirez, M. A., Salguero, F. J., Diaz San Segundo, F., Rabano, A., Cano, M. J., and Torres, J. M. (2004). Different behavior toward bovine spongiform encephalopathy infection of bovine prion protein transgenic mice with one extra repeat octapeptide insert mutation. $J$ Neurosci 24(9), 2156-64.

Caughey, B., and Kocisko, D. A. (2003). Prion diseases: a nucleic-acid accomplice? Nature 425(6959), 673-4.

Caughey, B., and Race, R. E. (1992). Potent inhibition of scrapie-associated PrP accumulation by congo red. J Neurochem 59(2), 768-71.

Caughey, B., and Raymond, G. J. (1991). The scrapie-associated form of PrP is made from a cell surface precursor that is both protease- and phospholipase-sensitive. J Biol Chem 266(27), 18217-23.

Caughey, B., and Raymond, G. J. (1993). Sulfated polyanion inhibition of scrapieassociated PrP accumulation in cultured cells. J Virol 67(2), 643-50.

Caughey, B., Raymond, G. J., Ernst, D., and Race, R. E. (1991). N-terminal truncation of the scrapie-associated form of PrP by lysosomal protease(s): implications regarding the site of conversion of PrP to the protease-resistant state. J Virol 65(12), 6597603.

Chen, S. G., Teplow, D. B., Parchi, P., Teller, J. K., Gambetti, P., and Autilio-Gambetti, L. (1995). Truncated forms of the human prion protein in normal brain and in prion diseases. J Biol Chem 270(32), 19173-80.

Chomczynski, P. (1993). A reagent for the single-step simultaneous isolation of RNA, DNA and proteins from cell and tissue samples. Biotechniques 15(3), 532-4, 5367.

Chomczynski, P., and Sacchi, N. (1987). Single-step method of RNA isolation by acid guanidinium thiocyanate-phenol-chloroform extraction. Anal Biochem 162(1), 156-9.

Clarke, M. C., and Haig, D. A. (1970). Evidence for the multiplication of scrapie agent in cell culture. Nature 225(5227), 100-1.

Clauss, M., Sauter-Louis, C., Chaher, E., Pottgiesser, C., Goebel, S., Selhorst, T., Wichmann, H. E., Klee, W., and Kienzle, E. (2006). Investigations of the potential risk factors associated with cases of bovine spongiform encephalopathy in Bavaria, Germany. Vet Rec 158(15), 509-13.

Clever, J., Sassetti, C., and Parslow, T. G. (1995). RNA secondary structure and binding sites for gag gene products in the 5 ' packaging signal of human immunodeficiency virus type 1.J Virol 69(4), 2101-9.

Cockcroft, P. D. (2004). The similarity of the physical sign frequencies of bovine spongiform encephalopathy and selected differential diagnoses. Vet J 167(2), 175-80.

Collinge, J. (1997). Human prion diseases and bovine spongiform encephalopathy (BSE). Hum Mol Genet 6(10), 1699-705. 
Collinge, J. (2005). Molecular neurology of prion disease. J Neurol Neurosurg Psychiatry 76(7), 906-19.

Collinge, J., Palmer, M. S., Sidle, K. C., Hill, A. F., Gowland, I., Meads, J., Asante, E., Bradley, R., Doey, L. J., and Lantos, P. L. (1995). Unaltered susceptibility to BSE in transgenic mice expressing human prion protein. Nature 378(6559), 779-83.

Collinge, J., Whitfield, J., McKintosh, E., Beck, J., Mead, S., Thomas, D. J., and Alpers, M. P. (2006). Kuru in the 21st century--an acquired human prion disease with very long incubation periods. Lancet 367(9528), 2068-74.

Collinge, J., Whitfield, J., McKintosh, E., Frosh, A., Mead, S., Hill, A. F., Brandner, S., Thomas, D., and Alpers, M. P. (2008). A clinical study of kuru patients with long incubation periods at the end of the epidemic in Papua New Guinea. Philos Trans $R$ Soc Lond B Biol Sci 363(1510), 3725-39.

Comoy, E. E., Casalone, C., Lescoutra-Etchegaray, N., Zanusso, G., Freire, S., Marce, D., Auvre, F., Ruchoux, M. M., Ferrari, S., Monaco, S., Sales, N., Caramelli, M., Leboulch, P., Brown, P., Lasmezas, C. I., and Deslys, J. P. (2008). Atypical BSE (BASE) transmitted from asymptomatic aging cattle to a primate. PLoS One 3(8), e3017.

Creutzfeldt, H. G. (1920). On a particular focal disease of the central nervous system (preliminary communication), 1920. Alzheimer Dis Assoc Disord 3(1-2), 3-25.

Croes, E. A., Theuns, J., Houwing-Duistermaat, J. J., Dermaut, B., Sleegers, K., Roks, G., Van den Broeck, M., van Harten, B., van Swieten, J. C., Cruts, M., Van Broeckhoven, C., and van Duijn, C. M. (2004). Octapeptide repeat insertions in the prion protein gene and early onset dementia. J Neurol Neurosurg Psychiatry 75(8), 1166-70.

Cronier, S., Laude, H., and Peyrin, J. M. (2004). Prions can infect primary cultured neurons and astrocytes and promote neuronal cell death. Proc Natl Acad Sci U S A 101(33), 12271-6.

Cuille, J., Chelle, P. (1939). Transmission experimentale de la tremblante chez la chevre. C. R. Acad. Sci. III(208), 1058-1060.

Darken, M. A. (1964). Puromycin Inhibition of Protein Synthesis. Pharmacol Rev 16, 22343.

Davis BJ, O. L. (1959). A new high resolution electrophoresis method. Delivered at the Society for the Study of Blood at the New York Academy of Medicine.

Dawson, M., Moore, R. C., and Bishop, S. C. (2008). Progress and limits of PrP gene selection policy. Vet Res 39(4), 25.

De Bosschere, H., Saegerman, C., Neukermans, A., Berkvens, D., Casaer, J., Vanopdenbosch, E., and Roels, S. (2006). First chronic wasting disease (CWD) surveillance of roe deer (Capreolus capreolus) in the northern part of Belgium. Vet $Q$ 28(2), 55-60.

Dealler, S., and Rainov, N. G. (2003). Pentosan polysulfate as a prophylactic and therapeutic agent against prion disease. IDrugs 6(5), 470-8.

DeArmond, S. J., Sanchez, H., Yehiely, F., Qiu, Y., Ninchak-Casey, A., Daggett, V., Camerino, A. P., Cayetano, J., Rogers, M., Groth, D., Torchia, M., Tremblay, P., Scott, M. R., Cohen, F. E., and Prusiner, S. B. (1997). Selective neuronal targeting in prion disease. Neuron 19(6), 1337-48.

DEFRA (2000). Report of the BSE inquiry published. Commun Dis Rep CDR Wkly 10(44), $391,394$.

Deleault, N. R., Lucassen, R. W., and Supattapone, S. (2003). RNA molecules stimulate prion protein conversion. Nature 425(6959), 717-20.

Di Bari, M. A., Chianini, F., Vaccari, G., Esposito, E., Conte, M., Eaton, S. L., Hamilton, S., Finlayson, J., Steele, P. J., Dagleish, M. P., Reid, H. W., Bruce, M., Jeffrey, M., Agrimi, 
U., and Nonno, R. (2008). The bank vole (Myodes glareolus) as a sensitive bioassay for sheep scrapie. J Gen Virol 89(Pt 12), 2975-85.

Diener, T. O., McKinley, M. P., and Prusiner, S. B. (1982). Viroids and prions. Proc Natl Acad Sci U S A 79(17), 5220-4.

Douglas, G. (2011). Workshop on Chronic Wasting Disease, Edmonton.

Ebeling, W., Hennrich, N., Klockow, M., Metz, H., Orth, H. D., and Lang, H. (1974). Proteinase K from Tritirachium album Limber. Eur J Biochem 47(1), 91-7.

Edenhofer, F., Rieger, R., Famulok, M., Wendler, W., Weiss, S., and Winnacker, E. L. (1996). Prion protein PrPc interacts with molecular chaperones of the Hsp60 family. J Virol 70(7), 4724-8.

Edgeworth, J. A., Farmer, M., Sicilia, A., Tavares, P., Beck, J., Campbell, T., Lowe, J., Mead, S., Rudge, P., Collinge, J., and Jackson, G. S. (2011). Detection of prion infection in variant Creutzfeldt-Jakob disease: a blood-based assay. Lancet 377(9764), 48793.

Edgeworth, J. A., Jackson, G. S., Clarke, A. R., Weissmann, C., and Collinge, J. (2009). Highly sensitive, quantitative cell-based assay for prions adsorbed to solid surfaces. Proc Natl Acad Sci U S A 106(9), 3479-83.

Enari, M., Flechsig, E., and Weissmann, C. (2001). Scrapie prion protein accumulation by scrapie-infected neuroblastoma cells abrogated by exposure to a prion protein antibody. Proc Natl Acad Sci U S A 98(16), 9295-9.

Engvall, E., and Perlmann, P. (1971). Enzyme-linked immunosorbent assay (ELISA). Quantitative assay of immunoglobulin G. Immunochemistry 8(9), 871-4.

EUROCJD (2011). vCJD cases worldwide. European Creutzfeldt Jakob Disease Surveillance Network.

Fasano, C., Campana, V., and Zurzolo, C. (2006). Prions: protein only or something more? Overview of potential prion cofactors. J Mol Neurosci 29(3), 195-214.

Felgner, P. L., Gadek, T. R., Holm, M., Roman, R., Chan, H. W., Wenz, M., Northrop, J. P., Ringold, G. M., and Danielsen, M. (1987). Lipofection: a highly efficient, lipidmediated DNA-transfection procedure. Proc Natl Acad Sci U S A 84(21), 7413-7.

Feraudet, C., Morel, N., Simon, S., Volland, H., Frobert, Y., Creminon, C., Vilette, D., Lehmann, S., and Grassi, J. (2005). Screening of 145 anti-PrP monoclonal antibodies for their capacity to inhibit PrPSc replication in infected cells. J Biol Chem 280(12), 11247-58.

Fevrier, B., Vilette, D., Archer, F., Loew, D., Faigle, W., Vidal, M., Laude, H., and Raposo, G. (2004). Cells release prions in association with exosomes. Proc Natl Acad Sci US A 101(26), 9683-8.

Field, E. J. (1968). Transmission of kuru to mice. Lancet 1(7549), 981-2.

Foster, J. D., Bruce, M., McConnell, I., Chree, A., and Fraser, H. (1996). Detection of BSE infectivity in brain and spleen of experimentally infected sheep. Vet Rec 138(22), 546-8.

Foster, J. D., Hope, J., McConnell, I., Bruce, M., and Fraser, H. (1994). Transmission of bovine spongiform encephalopathy to sheep, goats, and mice. Ann N Y Acad Sci 724, 300-3.

Gajdusek, D. C., Gibbs, C. J., and Alpers, M. (1966). Experimental transmission of a Kurulike syndrome to chimpanzees. Nature 209(5025), 794-6.

Gajdusek, D. C., and Zigas, V. (1957). Degenerative disease of the central nervous system in New Guinea; the endemic occurrence of kuru in the native population. $N$ Engl J Med 257(20), 974-8.

Gambetti, P., and Parchi, P. (1999). Insomnia in prion diseases: sporadic and familial. $N$ Engl J Med 340(21), 1675-7. 
Garner, B., Baoutina, A., Dean, R. T., and Jessup, W. (1997). Regulation of serum-induced lipid accumulation in human monocyte-derived macrophages by interferongamma. Correlations with apolipoprotein E production, lipoprotein lipase activity and LDL receptor-related protein expression. Atherosclerosis 128(1), 47-58.

Gauczynski, S., Peyrin, J. M., Haik, S., Leucht, C., Hundt, C., Rieger, R., Krasemann, S., Deslys, J. P., Dormont, D., Lasmezas, C. I., and Weiss, S. (2001). The 37-kDa/67$\mathrm{kDa}$ laminin receptor acts as the cell-surface receptor for the cellular prion protein. Embo J 20(21), 5863-75.

Gerstmann, J., Sträussler, E., Scheinker, I. (1936). Über eine eigenartige hereditärfamiliäre Erkrankung des Zentralnervensystems. Zugleich ein Beitrag zur Frage des vorzeitigen lokalen Alterns. Zeitschrift für die gesamte Neurologie und Psychiatrie 154, 736-762.

Gibbs, C. J., Jr., Amyx, H. L., Bacote, A., Masters, C. L., and Gajdusek, D. C. (1980). Oral transmission of kuru, Creutzfeldt-Jakob disease, and scrapie to nonhuman primates. J Infect Dis 142(2), 205-8.

Gibbs, C. J., Jr., and Gajdusek, D. C. (1972). Transmission of scrapie to the cynomolgus monkey (Macaca fascicularis). Nature 236(5341), 73-4.

Gibbs, C. J., Jr., Gajdusek, D. C., Asher, D. M., Alpers, M. P., Beck, E., Daniel, P. M., and Matthews, W. B. (1968). Creutzfeldt-Jakob disease (spongiform encephalopathy): transmission to the chimpanzee. Science 161(839), 388-9.

Giese, A., Brown, D. R., Groschup, M. H., Feldmann, C., Haist, I., and Kretzschmar, H. A. (1998). Role of microglia in neuronal cell death in prion disease. Brain Pathol 8(3), 449-57.

Gonzalez, L., Dagleish, M. P., Martin, S., Dexter, G., Steele, P., Finlayson, J., and Jeffrey, M. (2008). Diagnosis of preclinical scrapie in live sheep by the immunohistochemical examination of rectal biopsies. Vet Rec 162(13), 397-403.

Gossert, A. D., Bonjour, S., Lysek, D. A., Fiorito, F., and Wuthrich, K. (2005). Prion protein NMR structures of elk and of mouse/elk hybrids. Proc Natl Acad Sci U S A 102(3), 646-50.

Gough, K. C., and Maddison, B. C. (2010). Prion transmission: prion excretion and occurrence in the environment. Prion 4(4), 275-82.

Gousset, K., Schiff, E., Langevin, C., Marijanovic, Z., Caputo, A., Browman, D. T., Chenouard, N., de Chaumont, F., Martino, A., Enninga, J., Olivo-Marin, J. C., Mannel, D., and Zurzolo, C. (2009). Prions hijack tunnelling nanotubes for intercellular spread. Nat Cell Biol 11(3), 328-36.

Graham, F. L., Smiley, J., Russell, W. C., and Nairn, R. (1977). Characteristics of a human cell line transformed by DNA from human adenovirus type 5. J Gen Virol 36(1), 59-74.

Gray, J. W., Dolbeare, F., Pallavicini, M. G., Beisker, W., and Waldman, F. (1986). Cell cycle analysis using flow cytometry. Int J Radiat Biol Relat Stud Phys Chem Med 49(2), 237-55.

Green, K. M., Browning, S. R., Seward, T. S., Jewell, J. E., Ross, D. L., Green, M. A., Williams, E. S., Hoover, E. A., and Telling, G. C. (2008). The elk PRNP codon 132 polymorphism controls cervid and scrapie prion propagation. J Gen Virol 89(Pt 2), 598-608.

Gregory, L. G., Harbottle, R. P., Lawrence, L., Knapton, H. J., Themis, M., and Coutelle, C. (2003). Enhancement of adenovirus-mediated gene transfer to the airways by DEAE dextran and sodium caprate in vivo. Mol Ther 7(1), 19-26. 
Hall, M. P., Burson, K. K., and Huestis, W. H. (1998). Interactions of a vesicular stomatitis virus $\mathrm{G}$ protein fragment with phosphatidylserine: NMR and fluorescence studies. Biochim Biophys Acta 1415(1), 101-13.

Hamir, A. N., Cutlip, R. C., Miller, J. M., Williams, E. S., Stack, M. J., Miller, M. W., O'Rourke, K. I., and Chaplin, M. J. (2001). Preliminary findings on the experimental transmission of chronic wasting disease agent of mule deer to cattle. J Vet Diagn Invest 13(1), 91-6.

Hamir, A. N., Miller, J. M., Cutlip, R. C., Kunkle, R. A., Jenny, A. L., Stack, M. J., Chaplin, M. J., and Richt, J. A. (2004a). Transmission of sheep scrapie to elk (Cervus elaphus nelsoni) by intracerebral inoculation: final outcome of the experiment. $J$ Vet Diagn Invest 16(4), 316-21.

Hamir, A. N., Miller, J. M., O'Rourke, K. I., Bartz, J. C., Stack, M. J., and Chaplin, M. J. (2004b). Transmission of transmissible mink encephalopathy to raccoons (Procyon lotor) by intracerebral inoculation. J Vet Diagn Invest 16(1), 57-63.

Hamir, A. N., Richt, J. A., Miller, J. M., Kunkle, R. A., Hall, S. M., Nicholson, E. M., O'Rourke, K. I., Greenlee, J. J., and Williams, E. S. (2008). Experimental transmission of chronic wasting disease (CWD) of elk (Cervus elaphus nelsoni), white-tailed deer (Odocoileus virginianus), and mule deer (Odocoileus hemionus hemionus) to white-tailed deer by intracerebral route. Vet Pathol 45(3), 297-306.

Hardt, M., Baron, T., and Groschup, M. H. (2000). A comparative study of immunohistochemical methods for detecting abnormal prion protein with monoclonal and polyclonal antibodies. J Comp Pathol 122(1), 43-53.

Hayasaka, K., Gojobori, T., and Horai, S. (1988). Molecular phylogeny and evolution of primate mitochondrial DNA. Mol Biol Evol 5(6), 626-44.

Heisey, D. M., Mickelsen, N. A., Schneider, J. R., Johnson, C. J., Johnson, C. J., Langenberg, J. A., Bochsler, P. N., Keane, D. P., and Barr, D. J. (2010). Chronic wasting disease (CWD) susceptibility of several North American rodents that are sympatric with cervid CWD epidemics. J Virol 84(1), 210-5.

Herms, J., Tings, T., Gall, S., Madlung, A., Giese, A., Siebert, H., Schurmann, P., Windl, O., Brose, N., and Kretzschmar, H. (1999). Evidence of presynaptic location and function of the prion protein. J Neurosci 19(20), 8866-75.

Herzog, C., Riviere, J., Lescoutra-Etchegaray, N., Charbonnier, A., Leblanc, V., Sales, N., Deslys, J. P., and Lasmezas, C. I. (2005). PrPTSE distribution in a primate model of variant, sporadic, and iatrogenic Creutzfeldt-Jakob disease. J Virol 79(22), 1433945.

Herzog, C., Sales, N., Etchegaray, N., Charbonnier, A., Freire, S., Dormont, D., Deslys, J. P., and Lasmezas, C. I. (2004). Tissue distribution of bovine spongiform encephalopathy agent in primates after intravenous or oral infection. Lancet 363(9407), 422-8.

Hillion, J., Canals, M., Torvinen, M., Casado, V., Scott, R., Terasmaa, A., Hansson, A., Watson, S., Olah, M. E., Mallol, J., Canela, E. I., Zoli, M., Agnati, L. F., Ibanez, C. F., Lluis, C., Franco, R., Ferre, S., and Fuxe, K. (2002). Coaggregation, cointernalization, and codesensitization of adenosine $\mathrm{A} 2 \mathrm{~A}$ receptors and dopamine D2 receptors. J Biol Chem 277(20), 18091-7.

Hirose, K., Iwasaki, Y., Izumi, M., Yoshida, M., Hashizume, Y., Kitamoto, T., and Sahashi, K. (2006). MM2-thalamic-type sporadic Creutzfeldt-Jakob disease with widespread neocortical pathology. Acta Neuropathol 112(4), 503-11.

Holznagel, E., Yutzy, B., Schulz-Schaeffer, W., Hanschman, K. M., Stuke, A., Hahmann, U., Torner, M., Coulibaly, C., Hoffmann, A., Hunsmann, G., and Lower, J. (2010). Increase in CD230 (cellular prion protein) fluorescence on blood lymphocytes in 
bovine spongiform encephalopathy-infected nonhuman primates. Transfusion $\mathbf{5 0}(2), 452-66$.

Hornabrook, R. W. (1968). Kuru--a subacute cerebellar degeneration. The natural history and clinical features. Brain 91(1), 53-74.

Hornabrook, R. W., and Field, E. J. (1969). Clinical and pathological findings in kuru. Lancet 1(7594), 576.

Hunter, N., Goldmann, W., Smith, G., and Hope, J. (1994). Frequencies of PrP gene variants in healthy cattle and cattle with BSE in Scotland. Vet Rec 135(17), 400-3.

Ironside, J. W., and Head, M. W. (2004). Neuropathology and molecular biology of variant Creutzfeldt-Jakob disease. Curr Top Microbiol Immunol 284, 133-59.

Iwasaki, Y., Iijima, M., Kimura, S., Yoshida, M., Hashizume, Y., Yamada, M., Kitamoto, T., and Sobue, G. (2006). Autopsy case of sporadic Creutzfeldt-Jakob disease presenting with signs suggestive of brainstem and spinal cord involvement. Neuropathology 26(6), 550-6.

Jackson, G. S., and Clarke, A. R. (2000). Mammalian prion proteins. Curr Opin Struct Biol 10(1), 69-74.

Jakob, A. M. (1921). Über eine eigenartige Erkrankung des Zentralnervensystems mit bemerkenswerten anatomischen Befunden. Dtsch Z Nervenheilk 70, 132-46.

Jansen, C., Parchi, P., Capellari, S., Strammiello, R., Dopper, E. G., van Swieten, J. C., Kamphorst, W., and Rozemuller, A. J. (2011). A Second Case of GerstmannStraussler-Scheinker Disease Linked to the G131V Mutation in the Prion Protein Gene in a Dutch Patient. J Neuropathol Exp Neurol 70(8), 698-702.

Jendroska, K., Heinzel, F. P., Torchia, M., Stowring, L., Kretzschmar, H. A., Kon, A., Stern, A., Prusiner, S. B., and DeArmond, S. J. (1991). Proteinase-resistant prion protein accumulation in Syrian hamster brain correlates with regional pathology and scrapie infectivity. Neurology 41(9), 1482-90.

Jewell, J. E., Brown, J., Kreeger, T., and Williams, E. S. (2006). Prion protein in cardiac muscle of elk (Cervus elaphus nelsoni) and white-tailed deer (Odocoileus virginianus) infected with chronic wasting disease. J Gen Virol 87(Pt 11), 344350.

Johnson, C., Johnson, J., Clayton, M., McKenzie, D., and Aiken, J. (2003). Prion protein gene heterogeneity in free-ranging white-tailed deer within the chronic wasting disease affected region of Wisconsin. J Wildl Dis 39(3), 576-81.

Johnson, C. J., Bennett, J. P., Biro, S. M., Duque-Velasquez, J. C., Rodriguez, C. M., Bessen, R. A., and Rocke, T. E. (2011). Degradation of the disease-associated prion protein by a serine protease from lichens. PLoS One 6(5), e19836.

Keane, D., Barr, D., Osborn, R., Langenberg, J., O'Rourke, K., Schneider, D., and Bochsler, P. (2009). Validation of use of rectoanal mucosa-associated lymphoid tissue for immunohistochemical diagnosis of chronic wasting disease in white-tailed deer (Odocoileus virginianus). J Clin Microbiol 47(5), 1412-7.

Kim, Y. S., Carp, R. I., Callahan, S. M., Natelli, M., and Wisniewski, H. M. (1990a). Vacuolization, incubation period and survival time analyses in three mouse genotypes injected stereotactically in three brain regions with the $22 \mathrm{~L}$ scrapie strain. J Neuropathol Exp Neurol 49(2), 106-13.

Kim, Y. S., Carp, R. I., Callahan, S. M., and Wisniewski, H. M. (1990b). Pathogenesis and pathology of scrapie after stereotactic injection of strain 22L in intact and bisected cerebella. J Neuropathol Exp Neurol 49(2), 114-21.

Kirkwood, J. K., and Cunningham, A. A. (1994). Epidemiological observations on spongiform encephalopathies in captive wild animals in the British Isles. Vet Rec 135(13), 296-303. 
Klingeborn, M., Race, B., Meade-White, K. D., Rosenke, R., Striebel, J. F., and Chesebro, B. (2011). Crucial role for prion protein membrane anchoring in the neuroinvasion and neural spread of prion infection. J Virol 85(4), 1484-94.

Klohn, P. C., Stoltze, L., Flechsig, E., Enari, M., and Weissmann, C. (2003). A quantitative, highly sensitive cell-based infectivity assay for mouse scrapie prions. Proc Natl Acad Sci U S A 100(20), 11666-71.

Kocisko, D. A., Baron, G. S., Rubenstein, R., Chen, J., Kuizon, S., and Caughey, B. (2003). New inhibitors of scrapie-associated prion protein formation in a library of 2000 drugs and natural products. J Virol 77(19), 10288-94.

Kocisko, D. A., Priola, S. A., Raymond, G. J., Chesebro, B., Lansbury, P. T., Jr., and Caughey, B. (1995). Species specificity in the cell-free conversion of prion protein to protease-resistant forms: a model for the scrapie species barrier. Proc Natl Acad Sci U S A 92(9), 3923-7.

Kocisko, D. A., Vaillant, A., Lee, K. S., Arnold, K. M., Bertholet, N., Race, R. E., Olsen, E. A., Juteau, J. M., and Caughey, B. (2006). Potent antiscrapie activities of degenerate phosphorothioate oligonucleotides. Antimicrob Agents Chemother 50(3), 103444.

Kong, Q., Zheng, M., Casalone, C., Qing, L., Huang, S., Chakraborty, B., Wang, P., Chen, F., Cali, I., Corona, C., Martucci, F., Iulini, B., Acutis, P., Wang, L., Liang, J., Wang, M., Li, X., Monaco, S., Zanusso, G., Zou, W. Q., Caramelli, M., and Gambetti, P. (2008). Evaluation of the human transmission risk of an atypical bovine spongiform encephalopathy prion strain. J Virol 82(7), 3697-701.

Korth, C., Kaneko, K., and Prusiner, S. B. (2000). Expression of unglycosylated mutated prion protein facilitates $\operatorname{PrP}(\mathrm{Sc})$ formation in neuroblastoma cells infected with different prion strains. J Gen Virol 81(Pt 10), 2555-63.

Kovacs, G. G., Trabattoni, G., Hainfellner, J. A., Ironside, J. W., Knight, R. S., and Budka, H. (2002). Mutations of the prion protein gene phenotypic spectrum. J Neurol 249(11), 1567-82.

Kralovicova, S., Fontaine, S. N., Alderton, A., Alderman, J., Ragnarsdottir, K. V., Collins, S. J., and Brown, D. R. (2009). The effects of prion protein expression on metal metabolism. Mol Cell Neurosci 41(2), 135-47.

Kramer, M. L., Kratzin, H. D., Schmidt, B., Romer, A., Windl, O., Liemann, S., Hornemann, S., and Kretzschmar, H. (2001). Prion protein binds copper within the physiological concentration range. J Biol Chem 276(20), 16711-9.

Krasemann, S., Groschup, M., Hunsmann, G., and Bodemer, W. (1996a). Induction of antibodies against human prion proteins (PrP) by DNA-mediated immunization of PrP0/0 mice. J Immunol Methods 199(2), 109-18.

Krasemann, S., Groschup, M. H., Harmeyer, S., Hunsmann, G., and Bodemer, W. (1996b). Generation of monoclonal antibodies against human prion proteins in PrP0/0 mice. Mol Med 2(6), 725-34.

Kubler, E., Oesch, B., and Raeber, A. J. (2003). Diagnosis of prion diseases. Br Med Bull 66, 267-79.

Kupfer, L., Hinrichs, W., and Groschup, M. H. (2009). Prion protein misfolding. Curr Mol Med 9(7), 826-35.

Kuwahara, C., Takeuchi, A. M., Nishimura, T., Haraguchi, K., Kubosaki, A., Matsumoto, Y., Saeki, K., Matsumoto, Y., Yokoyama, T., Itohara, S., and Onodera, T. (1999). Prions prevent neuronal cell-line death. Nature 400(6741), 225-6.

Ladogana, A., Liu, Q., Xi, Y. G., and Pocchiari, M. (1995). Proteinase-resistant protein in human neuroblastoma cells infected with brain material from Creutzfeldt-Jakob patient. Lancet 345(8949), 594-5. 
Laemmli, U. K. (1970). Cleavage of structural proteins during the assembly of the head of bacteriophage T4. Nature 227(5259), 680-5.

Laine, J., Marc, M. E., Sy, M. S., and Axelrad, H. (2001). Cellular and subcellular morphological localization of normal prion protein in rodent cerebellum. Eur J Neurosci 14(1), 47-56.

Lasmezas, C. I., Comoy, E., Hawkins, S., Herzog, C., Mouthon, F., Konold, T., Auvre, F., Correia, E., Lescoutra-Etchegaray, N., Sales, N., Wells, G., Brown, P., and Deslys, J. P. (2005). Risk of oral infection with bovine spongiform encephalopathy agent in primates. Lancet 365(9461), 781-3.

Lasmezas, C. I., Deslys, J. P., Demaimay, R., Adjou, K. T., Lamoury, F., Dormont, D., Robain, O., Ironside, J., and Hauw, J. J. (1996). BSE transmission to macaques. Nature 381(6585), 743-4.

Lawson, V. A., Collins, S. J., Masters, C. L., and Hill, A. F. (2005). Prion protein glycosylation. J Neurochem 93(4), 793-801.

Le Dur, A., Beringue, V., Andreoletti, O., Reine, F., Lai, T. L., Baron, T., Bratberg, B., Vilotte, J. L., Sarradin, P., Benestad, S. L., and Laude, H. (2005). A newly identified type of scrapie agent can naturally infect sheep with resistant PrP genotypes. Proc Natl Acad Sci U S A 102(44), 16031-6.

Leblanc, P., Alais, S., Porto-Carreiro, I., Lehmann, S., Grassi, J., Raposo, G., and Darlix, J. L. (2006). Retrovirus infection strongly enhances scrapie infectivity release in cell culture. Embo J 25(12), 2674-85.

Leucht, C., Simoneau, S., Rey, C., Vana, K., Rieger, R., Lasmezas, C. I., and Weiss, S. (2003). The $37 \mathrm{kDa} / 67 \mathrm{kDa}$ laminin receptor is required for $\operatorname{PrP}(\mathrm{Sc})$ propagation in scrapie-infected neuronal cells. EMBO Rep 4(3), 290-5.

Liberski, P. P., and Budka, H. (2004). Gerstmann-Straussler-Scheinker disease. I. Human diseases. Folia Neuropathol 42 Suppl B, 120-40.

Lima, L. M., Cordeiro, Y., Tinoco, L. W., Marques, A. F., Oliveira, C. L., Sampath, S., Kodali, R., Choi, G., Foguel, D., Torriani, I., Caughey, B., and Silva, J. L. (2006). Structural insights into the interaction between prion protein and nucleic acid. Biochemistry 45(30), 9180-7.

Linden, R., Martins, V. R., Prado, M. A., Cammarota, M., Izquierdo, I., and Brentani, R. R. (2008). Physiology of the prion protein. Physiol Rev 88(2), 673-728.

Liu, T., Zwingman, T., Li, R., Pan, T., Wong, B. S., Petersen, R. B., Gambetti, P., Herrup, K., and Sy, M. S. (2001). Differential expression of cellular prion protein in mouse brain as detected with multiple anti-PrP monoclonal antibodies. Brain Res 896(12), 118-29.

Llewelyn, C. A., Hewitt, P. E., Knight, R. S., Amar, K., Cousens, S., Mackenzie, J., and Will, R. G. (2004). Possible transmission of variant Creutzfeldt-Jakob disease by blood transfusion. Lancet 363(9407), 417-21.

Lugaresi, E., Medori, R., Montagna, P., Baruzzi, A., Cortelli, P., Lugaresi, A., Tinuper, P., Zucconi, M., and Gambetti, P. (1986). Fatal familial insomnia and dysautonomia with selective degeneration of thalamic nuclei. $N$ Engl J Med 315(16), 997-1003.

Luhr, K. M., Nordstrom, E. K., Low, P., and Kristensson, K. (2004a). Cathepsin B and L are involved in degradation of prions in GT1-1 neuronal cells. Neuroreport 15(10), 1663-7.

Luhr, K. M., Nordstrom, E. K., Low, P., Ljunggren, H. G., Taraboulos, A., and Kristensson, K. (2004b). Scrapie protein degradation by cysteine proteases in CD11c+ dendritic cells and GT1-1 neuronal cells. J Virol 78(9), 4776-82.

Lysek, D. A., Schorn, C., Nivon, L. G., Esteve-Moya, V., Christen, B., Calzolai, L., von Schroetter, C., Fiorito, F., Herrmann, T., Guntert, P., and Wuthrich, K. (2005). Prion 
protein NMR structures of cats, dogs, pigs, and sheep. Proc Natl Acad Sci U S A 102(3), 640-5.

Maas, E., Geissen, M., Groschup, M. H., Rost, R., Onodera, T., Schatzl, H., and Vorberg, I. M. (2007). Scrapie infection of prion protein-deficient cell line upon ectopic expression of mutant prion proteins. J Biol Chem 282(26), 18702-10.

Madec, J. Y., Vanier, A., Dorier, A., Bernillon, J., Belli, P., and Baron, T. (1997). Biochemical properties of protease resistant prion protein PrPsc in natural sheep scrapie. Arch Virol 142(8), 1603-12.

Mahal, S. P., Baker, C. A., Demczyk, C. A., Smith, E. W., Julius, C., and Weissmann, C. (2007). Prion strain discrimination in cell culture: the cell panel assay. Proc Natl Acad Sci U S A 104(52), 20908-13.

Mange, A., Nishida, N., Milhavet, O., McMahon, H. E., Casanova, D., and Lehmann, S. (2000). Amphotericin B inhibits the generation of the scrapie isoform of the prion protein in infected cultures. J Virol 74(7), 3135-40.

Manuelidis, L. (1997). Decontamination of Creutzfeldt-Jakob disease and other transmissible agents. J Neurovirol 3(1), 62-5.

Marques, A. F., Cordeiro, Y., Silva, J. L., and Lima, L. M. (2009). Enhanced prion protein stability coupled to DNA recognition and milieu acidification. Biophys Chem 141(2-3), 135-9.

Marsh, R. F., Burger, D., Eckroade, R., Zu Rhein, G. M., and Hanson, R. P. (1969). A preliminary report on the experimental host range of the transmissible mink encephalopathy agent. J Infect Dis 120(6), 713-9.

Marsh, R. F., Kincaid, A. E., Bessen, R. A., and Bartz, J. C. (2005). Interspecies transmission of chronic wasting disease prions to squirrel monkeys (Saimiri sciureus). J Virol 79(21), 13794-6.

Martucci, F., Acutis, P., Mazza, M., Nodari, S., Colussi, S., Corona, C., Barocci, S., Gabrielli, A., Caramelli, M., Casalone, C., and Moroncini, G. (2009). Detection of typical and atypical bovine spongiform encephalopathy and scrapie prion strains by prion protein motif-grafted antibodies. J Gen Virol 90(Pt 4), 1048-53.

Masliah, E., Rockenstein, E., Veinbergs, I., Mallory, M., Hashimoto, M., Takeda, A., Sagara, Y., Sisk, A., and Mucke, L. (2000). Dopaminergic loss and inclusion body formation in alpha-synuclein mice: implications for neurodegenerative disorders. Science 287(5456), 1265-9.

Masters, C. L., Harris, J. O., Gajdusek, D. C., Gibbs, C. J., Jr., Bernoulli, C., and Asher, D. M. (1979). Creutzfeldt-Jakob disease: patterns of worldwide occurrence and the significance of familial and sporadic clustering. Ann Neurol 5(2), 177-88.

Mastrianni, J. A. (1998). The prion diseases: Creutzfeldt-Jakob, Gerstmann-StrausslerScheinker, and related disorders. J Geriatr Psychiatry Neurol 11(2), 78-97.

Mastromarino, P., Conti, C., Goldoni, P., Hauttecoeur, B., and Orsi, N. (1987). Characterization of membrane components of the erythrocyte involved in vesicular stomatitis virus attachment and fusion at acidic pH. J Gen Virol 68 ( Pt 9), 2359-69.

Mathiason, C. K., Powers, J. G., Dahmes, S. J., Osborn, D. A., Miller, K. V., Warren, R. J., Mason, G. L., Hays, S. A., Hayes-Klug, J., Seelig, D. M., Wild, M. A., Wolfe, L. L., Spraker, T. R., Miller, M. W., Sigurdson, C. J., Telling, G. C., and Hoover, E. A. (2006). Infectious prions in the saliva and blood of deer with chronic wasting disease. Science 314(5796), 133-6.

May, B. C., Fafarman, A. T., Hong, S. B., Rogers, M., Deady, L. W., Prusiner, S. B., and Cohen, F. E. (2003). Potent inhibition of scrapie prion replication in cultured cells by bisacridines. Proc Natl Acad Sci U S A 100(6), 3416-21. 
Mays, C. E., and Ryou, C. (2011). Plasminogen: A cellular protein cofactor for PrPSc propagation. Prion 5(1), 22-7.

McGowan, J. P. (1922). Scrapie in Sheep. Scottish Journal of Agriculture 5, 365-75.

McKinley, M. P., Bolton, D. C., and Prusiner, S. B. (1983). A protease-resistant protein is a structural component of the scrapie prion. Cell 35(1), 57-62.

McNally, K. L., Ward, A. E., and Priola, S. A. (2009). Cells expressing anchorless prion protein are resistant to scrapie infection. J Virol 83(9), 4469-75.

Mead, S. (2006). Prion disease genetics. Eur J Hum Genet 14(3), 273-81.

Meade-White, K., Race, B., Trifilo, M., Bossers, A., Favara, C., Lacasse, R., Miller, M., Williams, E., Oldstone, M., Race, R., and Chesebro, B. (2007). Resistance to chronic wasting disease in transgenic mice expressing a naturally occurring allelic variant of deer prion protein. J Virol 81(9), 4533-9.

Medori, R., Montagna, P., Tritschler, H. J., LeBlanc, A., Cortelli, P., Tinuper, P., Lugaresi, E., and Gambetti, P. (1992). Fatal familial insomnia: a second kindred with mutation of prion protein gene at codon 178. Neurology 42(3 Pt 1), 669-70.

Milhavet, O., Casanova, D., Chevallier, N., McKay, R. D., and Lehmann, S. (2006). Neural stem cell model for prion propagation. Stem Cells 24(10), 2284-91.

Miller, A. D. (1997). Development and Applications of Retroviral Vectors.

Miller, M. W., Wild, M. A., and Williams, E. S. (1998). Epidemiology of chronic wasting disease in captive Rocky Mountain elk. J Wildl Dis 34(3), 532-8.

Miller, M. W., and Williams, E. S. (2003). Prion disease: horizontal prion transmission in mule deer. Nature 425(6953), 35-6.

Miller, M. W., and Williams, E. S. (2004). Chronic wasting disease of cervids. Curr Top Microbiol Immunol 284, 193-214.

Miller, M. W., Williams, E. S., Hobbs, N. T., and Wolfe, L. L. (2004). Environmental sources of prion transmission in mule deer. Emerg Infect Dis 10(6), 1003-6.

Montag, J., Hitt, R., Opitz, L., Schulz-Schaeffer, W. J., Hunsmann, G., and Motzkus, D. (2009). Upregulation of miRNA hsa-miR-342-3p in experimental and idiopathic prion disease. Mol Neurodegener 4, 36.

Montagna, P., Gambetti, P., Cortelli, P., and Lugaresi, E. (2003). Familial and sporadic fatal insomnia. Lancet Neurol 2(3), 167-76.

Morgenstern, J. P., and Land, H. (1990). Advanced mammalian gene transfer: high titre retroviral vectors with multiple drug selection markers and a complementary helper-free packaging cell line. Nucleic Acids Res 18(12), 3587-96.

Mullis, K., Faloona, F., Scharf, S., Saiki, R., Horn, G., and Erlich, H. (1986). Specific enzymatic amplification of DNA in vitro: the polymerase chain reaction. Cold Spring Harb Symp Quant Biol 51 Pt 1, 263-73.

Naldini, L., Blomer, U., Gallay, P., Ory, D., Mulligan, R., Gage, F. H., Verma, I. M., and Trono, D. (1996). In vivo gene delivery and stable transduction of nondividing cells by a lentiviral vector. Science 272(5259), 263-7.

Narayanan, K., and Makino, S. (2001). Cooperation of an RNA packaging signal and a viral envelope protein in coronavirus RNA packaging. J Virol 75(19), 9059-67.

Nathanson, N., Wilesmith, J., and Griot, C. (1997). Bovine spongiform encephalopathy (BSE): causes and consequences of a common source epidemic. Am J Epidemiol 145(11), 959-69.

Neale, M. H., Mountjoy, S. J., Edwards, J. C., Vilette, D., Laude, H., Windl, O., and Saunders, G. C. (2010). Infection of cell lines with experimental and natural ovine scrapie agents. J Virol 84(5), 2444-52. 
Neary, K., Caughey, B., Ernst, D., Race, R. E., and Chesebro, B. (1991). Protease sensitivity and nuclease resistance of the scrapie agent propagated in vitro in neuroblastoma cells. J Virol 65(2), 1031-4.

Neuendorf, E., Weber, A., Saalmueller, A., Schatzl, H., Reifenberg, K., Pfaff, E., and Groschup, M. H. (2004). Glycosylation deficiency at either one of the two glycan attachment sites of cellular prion protein preserves susceptibility to bovine spongiform encephalopathy and scrapie infections. J Biol Chem 279(51), 5330616.

Nishida, N., Harris, D. A., Vilette, D., Laude, H., Frobert, Y., Grassi, J., Casanova, D., Milhavet, O., and Lehmann, S. (2000). Successful transmission of three mouseadapted scrapie strains to murine neuroblastoma cell lines overexpressing wildtype mouse prion protein. J Virol 74(1), 320-5.

Novakofski, J., Brewer, M. S., Mateus-Pinilla, N., Killefer, J., and McCusker, R. H. (2005). Prion biology relevant to bovine spongiform encephalopathy. J Anim Sci 83(6), 1455-76.

Nunziante, M., Ackermann, K., Dietrich, K., Wolf, H., Gadtke, L., Gilch, S., Vorberg, I., Groschup, M., and Schatzl, H. M. (2011). Proteasomal dysfunction and ER stress enhance trafficking of prion protein aggregates through the secretory pathway and increase accumulation of PrPSc. J Biol Chem.

O'Rourke, K. I., Baszler, T. V., Besser, T. E., Miller, J. M., Cutlip, R. C., Wells, G. A., Ryder, S. J., Parish, S. M., Hamir, A. N., Cockett, N. E., Jenny, A., and Knowles, D. P. (2000). Preclinical diagnosis of scrapie by immunohistochemistry of third eyelid lymphoid tissue. J Clin Microbiol 38(9), 3254-9.

O'Rourke, K. I., Besser, T. E., Miller, M. W., Cline, T. F., Spraker, T. R., Jenny, A. L., Wild, M. A., Zebarth, G. L., and Williams, E. S. (1999). PrP genotypes of captive and freeranging Rocky Mountain elk (Cervus elaphus nelsoni) with chronic wasting disease. J Gen Virol 80 ( Pt 10), 2765-9.

Oesch, B., Westaway, D., Walchli, M., McKinley, M. P., Kent, S. B., Aebersold, R., Barry, R. A., Tempst, P., Teplow, D. B., Hood, L. E., and et al. (1985). A cellular gene encodes scrapie PrP 27-30 protein. Cell 40(4), 735-46.

Oliveira, C., de Souza, M., Saraiva, N., Tetzner, T., Lima, M., Lopes, F., and Garcia, J. (2011). In vitro Culture of Bovine Embryos in Murine ES Cell Conditioned Media Negatively Affects Expression of Pluripotency-Related Markers OCT4, SOX2 and SSEA1. Reprod Domest Anim.

Owen, F., Poulter, M., Collinge, J., and Crow, T. J. (1990). A codon 129 polymorphism in the PRIP gene. Nucleic Acids Res 18(10), 3103.

Pan, T., Wong, P., Chang, B., Li, C., Li, R., Kang, S. C., Wisniewski, T., and Sy, M. S. (2005). Biochemical fingerprints of prion infection: accumulations of aberrant full-length and N-terminally truncated PrP species are common features in mouse prion disease. J Virol 79(2), 934-43.

Parchi, P., Capellari, S., Chen, S. G., Petersen, R. B., Gambetti, P., Kopp, N., Brown, P., Kitamoto, T., Tateishi, J., Giese, A., and Kretzschmar, H. (1997). Typing prion isoforms. Nature 386(6622), 232-4.

Parchi, P., Castellani, R., Capellari, S., Ghetti, B., Young, K., Chen, S. G., Farlow, M., Dickson, D. W., Sima, A. A., Trojanowski, J. Q., Petersen, R. B., and Gambetti, P. (1996). Molecular basis of phenotypic variability in sporadic Creutzfeldt-Jakob disease. Ann Neurol 39(6), 767-78.

Parchi, P., Chen, S. G., Brown, P., Zou, W., Capellari, S., Budka, H., Hainfellner, J., Reyes, P. F., Golden, G. T., Hauw, J. J., Gajdusek, D. C., and Gambetti, P. (1998a). Different patterns of truncated prion protein fragments correlate with distinct phenotypes 
in P102L Gerstmann-Straussler-Scheinker disease. Proc Natl Acad Sci U S A 95(14), 8322-7.

Parchi, P., Giese, A., Capellari, S., Brown, P., Schulz-Schaeffer, W., Windl, O., Zerr, I., Budka, H., Kopp, N., Piccardo, P., Poser, S., Rojiani, A., Streichemberger, N., Julien, J., Vital, C., Ghetti, B., Gambetti, P., and Kretzschmar, H. (1999). Classification of sporadic Creutzfeldt-Jakob disease based on molecular and phenotypic analysis of 300 subjects. Ann Neurol 46(2), 224-33.

Parchi, P., Petersen, R. B., Chen, S. G., Autilio-Gambetti, L., Capellari, S., Monari, L., Cortelli, P., Montagna, P., Lugaresi, E., and Gambetti, P. (1998b). Molecular pathology of fatal familial insomnia. Brain Pathol 8(3), 539-48.

Peden, A. H., Head, M. W., Ritchie, D. L., Bell, J. E., and Ironside, J. W. (2004). Preclinical vCJD after blood transfusion in a PRNP codon 129 heterozygous patient. Lancet 364(9433), 527-9.

Peretz, D., Williamson, R. A., Kaneko, K., Vergara, J., Leclerc, E., Schmitt-Ulms, G., Mehlhorn, I. R., Legname, G., Wormald, M. R., Rudd, P. M., Dwek, R. A., Burton, D. R., and Prusiner, S. B. (2001). Antibodies inhibit prion propagation and clear cell cultures of prion infectivity. Nature 412(6848), 739-43.

Perrott, M. R., Sigurdson, C. J., Mason, G. L., and Hoover, E. A. (2011). Evidence for distinct CWD strains in experimental CWD in ferrets. J Gen Virol.

Piccardo, P., Dlouhy, S. R., Lievens, P. M., Young, K., Bird, T. D., Nochlin, D., Dickson, D. W., Vinters, H. V., Zimmerman, T. R., Mackenzie, I. R., Kish, S. J., Ang, L. C., De Carli, C., Pocchiari, M., Brown, P., Gibbs, C. J., Jr., Gajdusek, D. C., Bugiani, O., Ironside, J., Tagliavini, F., and Ghetti, B. (1998). Phenotypic variability of GerstmannStraussler-Scheinker disease is associated with prion protein heterogeneity. $J$ Neuropathol Exp Neurol 57(10), 979-88.

Piccardo, P., Ghetti, B., Dickson, D. W., Vinters, H. V., Giaccone, G., Bugiani, O., Tagliavini, F., Young, K., Dlouhy, S. R., Seiler, C., and et al. (1995). Gerstmann-StrausslerScheinker disease (PRNP P102L): amyloid deposits are best recognized by antibodies directed to epitopes in PrP region 90-165. J Neuropathol Exp Neurol 54(6), 790-801.

Piccardo, P., Liepnieks, J. J., William, A., Dlouhy, S. R., Farlow, M. R., Young, K., Nochlin, D., Bird, T. D., Nixon, R. R., Ball, M. J., DeCarli, C., Bugiani, O., Tagliavini, F., Benson, M. D., and Ghetti, B. (2001). Prion proteins with different conformations accumulate in Gerstmann-Straussler-Scheinker disease caused by A117V and F198S mutations. Am J Pathol 158(6), 2201-7.

Pick, E., Lau, O. S., Tsuge, T., Menon, S., Tong, Y., Dohmae, N., Plafker, S. M., Deng, X. W., and Wei, N. (2007). Mammalian DET1 regulates Cul4A activity and forms stable complexes with E2 ubiquitin-conjugating enzymes. Mol Cell Biol 27(13), 4708-19.

Pocchiari, M., Schmittinger, S., and Masullo, C. (1987). Amphotericin B delays the incubation period of scrapie in intracerebrally inoculated hamsters. J Gen Virol 68 ( Pt 1), 219-23.

Prasher, D. C., Eckenrode, V. K., Ward, W. W., Prendergast, F. G., and Cormier, M. J. (1992). Primary structure of the Aequorea victoria green-fluorescent protein. Gene 111(2), 229-33.

Prendergast, F. G., and Mann, K. G. (1978). Chemical and physical properties of aequorin and the green fluorescent protein isolated from Aequorea forskalea. Biochemistry 17(17), 3448-53.

Price, J., and Verner, K. (1993). Puromycin inhibits protein import into mitochondria by interfering with an intramitochondrial ATP-dependent reaction. Biochim Biophys Acta 1150(1), 89-97. 
Priola, S. A., and McNally, K. L. (2009). The role of the prion protein membrane anchor in prion infection. Prion 3(3), 134-8.

Provost, P. (2010). MicroRNAs as a molecular basis for mental retardation, Alzheimer's and prion diseases. Brain Res 1338, 58-66.

Prusiner, S. B. (1982). Novel proteinaceous infectious particles cause scrapie. Science 216(4542), 136-44.

Prusiner, S. B., Groth, D. F., McKinley, M. P., Cochran, S. P., Bowman, K. A., and Kasper, K. C. (1981). Thiocyanate and hydroxyl ions inactivate the scrapie agent. Proc Natl Acad Sci U S A 78(7), 4606-10.

Prusiner, S. B., Hadlow, W. J., Garfin, D. E., Cochran, S. P., Baringer, J. R., Race, R. E., and Eklund, C. M. (1978). Partial purification and evidence for multiple molecular forms of the scrapie agent. Biochemistry 17(23), 4993-9.

Prusiner, S. B., McKinley, M. P., Bowman, K. A., Bolton, D. C., Bendheim, P. E., Groth, D. F., and Glenner, G. G. (1983). Scrapie prions aggregate to form amyloid-like birefringent rods. Cell 35(2 Pt 1), 349-58.

Race, B., Meade-White, K. D., Miller, M. W., Barbian, K. D., Rubenstein, R., LaFauci, G., Cervenakova, L., Favara, C., Gardner, D., Long, D., Parnell, M., Striebel, J., Priola, S. A., Ward, A., Williams, E. S., Race, R., and Chesebro, B. (2009). Susceptibilities of nonhuman primates to chronic wasting disease. Emerg Infect Dis 15(9), 1366-76.

Race, R. E., Fadness, L. H., and Chesebro, B. (1987). Characterization of scrapie infection in mouse neuroblastoma cells. J Gen Virol 68 ( Pt 5), 1391-9.

Race, R. E., Priola, S. A., Bessen, R. A., Ernst, D., Dockter, J., Rall, G. F., Mucke, L., Chesebro, B., and Oldstone, M. B. (1995). Neuron-specific expression of a hamster prion protein minigene in transgenic mice induces susceptibility to hamster scrapie agent. Neuron 15(5), 1183-91.

Raymond, G. J., Olsen, E. A., Lee, K. S., Raymond, L. D., Bryant, P. K., 3rd, Baron, G. S., Caughey, W. S., Kocisko, D. A., McHolland, L. E., Favara, C., Langeveld, J. P., van Zijderveld, F. G., Mayer, R. T., Miller, M. W., Williams, E. S., and Caughey, B. (2006). Inhibition of protease-resistant prion protein formation in a transformed deer cell line infected with chronic wasting disease. J Virol 80(2), 596-604.

Raymond, G. J., Raymond, L. D., Meade-White, K. D., Hughson, A. G., Favara, C., Gardner, D., Williams, E. S., Miller, M. W., Race, R. E., and Caughey, B. (2007). Transmission and adaptation of chronic wasting disease to hamsters and transgenic mice: evidence for strains. J Virol 81(8), 4305-14.

Raymond, S., and Weintraub, L. (1959). Acrylamide gel as a supporting medium for zone electrophoresis. Science 130, 711.

Renart, J., Reiser, J., and Stark, G. R. (1979). Transfer of proteins from gels to diazobenzyloxymethyl-paper and detection with antisera: a method for studying antibody specificity and antigen structure. Proc Natl Acad Sci U S A 76(7), 311620.

Rieger, R., Edenhofer, F., Lasmezas, C. I., and Weiss, S. (1997). The human 37-kDa laminin receptor precursor interacts with the prion protein in eukaryotic cells. Nat Med 3(12), 1383-8.

Riek, R., Hornemann, S., Wider, G., Glockshuber, R., and Wuthrich, K. (1997). NMR characterization of the full-length recombinant murine prion protein, $\mathrm{mPrP}(23-$ 231). FEBS Lett 413(2), 282-8.

Rieseberg, M., Kasper, C., Reardon, K. F., and Scheper, T. (2001). Flow cytometry in biotechnology. Appl Microbiol Biotechnol 56(3-4), 350-60.

Riesner, D. (2003). Biochemistry and structure of $\operatorname{PrP}(\mathrm{C})$ and $\operatorname{PrP}(\mathrm{Sc}) . \mathrm{Br}$ Med Bull 66, 21-33. 
Roels, S., Saegerman, C., De Bosschere, H., Berkvens, D., Gregoire, F., Hoyoux, A., Mousset, B., Desmecht, D., Vanopdenbosch, E., and Linden, A. (2005). First results of chronic wasting disease (CWD) surveillance in the south-eastern part of Belgium. Vet $Q$ 27(3), 98-104.

Rogers, M., Taraboulos, A., Scott, M., Groth, D., and Prusiner, S. B. (1990). Intracellular accumulation of the cellular prion protein after mutagenesis of its Asn-linked glycosylation sites. Glycobiology 1(1), 101-9.

Rousculp, M. D., Goldsmith, K. T., and Garver, R. I., Jr. (1992). Quantitative evaluation of retroviral gene transduction efficiency in human lung cancer cells. Hum Gene Ther 3(5), 471-7.

Rudd, P. M., Wormald, M. R., Wing, D. R., Prusiner, S. B., and Dwek, R. A. (2001). Prion glycoprotein: structure, dynamics, and roles for the sugars. Biochemistry 40(13), 3759-66.

Rüdiger, J. F. (1710). Für das Draben. Der redliche und aufrichtige Schäfer, 208.

Russelakis-Carneiro, M., Saborio, G. P., Anderes, L., and Soto, C. (2002). Changes in the glycosylation pattern of prion protein in murine scrapie. Implications for the mechanism of neurodegeneration in prion diseases. J Biol Chem 277(39), 368727.

Saba, R., Goodman, C. D., Huzarewich, R. L., Robertson, C., and Booth, S. A. (2008). A miRNA signature of prion induced neurodegeneration. PLoS One 3(11), e3652.

Sabuncu, E., Petit, S., Le Dur, A., Lan Lai, T., Vilotte, J. L., Laude, H., and Vilette, D. (2003). PrP polymorphisms tightly control sheep prion replication in cultured cells. $J$ Virol 77(4), 2696-700.

Safar, J. G., Lessard, P., Tamguney, G., Freyman, Y., Deering, C., Letessier, F., Dearmond, S. J., and Prusiner, S. B. (2008). Transmission and detection of prions in feces. $J$ Infect Dis 198(1), 81-9.

Safar, J. G., Scott, M., Monaghan, J., Deering, C., Didorenko, S., Vergara, J., Ball, H., Legname, G., Leclerc, E., Solforosi, L., Serban, H., Groth, D., Burton, D. R., Prusiner, S. B., and Williamson, R. A. (2002). Measuring prions causing bovine spongiform encephalopathy or chronic wasting disease by immunoassays and transgenic mice. Nat Biotechnol 20(11), 1147-50.

Sandberg, M. K., Al-Doujaily, H., Sigurdson, C. J., Glatzel, M., O'Malley, C., Powell, C., Asante, E. A., Linehan, J. M., Brandner, S., Wadsworth, J. D., and Collinge, J. (2010). Chronic wasting disease prions are not transmissible to transgenic mice overexpressing human prion protein. J Gen Virol 91(Pt 10), 2651-7.

Sanger, F., Nicklen, S., and Coulson, A. R. (1977). DNA sequencing with chain-terminating inhibitors. Proc Natl Acad Sci U S A 74(12), 5463-7.

Schatzl, H. M., Laszlo, L., Holtzman, D. M., Tatzelt, J., DeArmond, S. J., Weiner, R. I., Mobley, W. C., and Prusiner, S. B. (1997). A hypothalamic neuronal cell line persistently infected with scrapie prions exhibits apoptosis. J Virol 71(11), 8821-31.

Schettler, E., Steinbach, F., Eschenbacher-Kaps, I., Gerst, K., Muessdoerffer, F., Risch, K., Streich, W. J., and Frolich, K. (2006). Surveillance for prion disease in cervids, Germany. Emerg Infect Dis 12(2), 319-22.

Schmitt, A., Meiners, I., Schmitt, J., Noller, J., Ihling, C., Munch, G., Sinz, A., and Nieber, K. (2005). Two analytical methods to study the interaction of AGEs with cell surface proteins. J Biochem Biophys Methods 65(2-3), 121-36.

Schonberger, O., Horonchik, L., Gabizon, R., Papy-Garcia, D., Barritault, D., and Taraboulos, A. (2003). Novel heparan mimetics potently inhibit the scrapie prion protein and its endocytosis. Biochem Biophys Res Commun 312(2), 473-9. 
Schreuder, B. E., van Keulen, L. J., Vromans, M. E., Langeveld, J. P., and Smits, M. A. (1998). Tonsillar biopsy and PrPSc detection in the preclinical diagnosis of scrapie. Vet Rec 142(21), 564-8.

Schulz-Schaeffer, W. J., Giese, A., Windl, O., and Kretzschmar, H. A. (1996). Polymorphism at codon 129 of the prion protein gene determines cerebellar pathology in Creutzfeldt-Jakob disease. Clin Neuropathol 15(6), 353-7.

Scott, M., Groth, D., Foster, D., Torchia, M., Yang, S. L., DeArmond, S. J., and Prusiner, S. B. (1993). Propagation of prions with artificial properties in transgenic mice expressing chimeric PrP genes. Cell 73(5), 979-88.

Scott, M. R., Peretz, D., Nguyen, H. O., Dearmond, S. J., and Prusiner, S. B. (2005). Transmission barriers for bovine, ovine, and human prions in transgenic mice. $J$ Virol 79(9), 5259-71.

Seelig, D. M., Mason, G. L., Telling, G. C., and Hoover, E. A. (2010). Pathogenesis of chronic wasting disease in cervidized transgenic mice. Am J Pathol 176(6), 2785-97.

Shimomura, O., Johnson, F. H., and Saiga, Y. (1962). Extraction, purification and properties of aequorin, a bioluminescent protein from the luminous hydromedusan, Aequorea. J Cell Comp Physiol 59, 223-39.

Shoemaker, C., Hoffman, J., Goff, S. P., and Baltimore, D. (1981). Intramolecular integration within Moloney murine leukemia virus DNA. J Virol 40(1), 164-72.

Sigurdson, C. J., Williams, E. S., Miller, M. W., Spraker, T. R., O'Rourke, K. I., and Hoover, E. A. (1999). Oral transmission and early lymphoid tropism of chronic wasting disease PrPres in mule deer fawns (Odocoileus hemionus). J Gen Virol 80 ( Pt 10), 2757-64.

Silva, J. L., Gomes, M. P., Vieira, T. C., and Cordeiro, Y. (2010). PrP interactions with nucleic acids and glycosaminoglycans in function and disease. Front Biosci 15, 132-50.

Silveira, J. R., Raymond, G. J., Hughson, A. G., Race, R. E., Sim, V. L., Hayes, S. F., and Caughey, B. (2005). The most infectious prion protein particles. Nature 437(7056), 257-61.

Simmons, M. M., Harris, P., Jeffrey, M., Meek, S. C., Blamire, I. W., and Wells, G. A. (1996). BSE in Great Britain: consistency of the neurohistopathological findings in two random annual samples of clinically suspect cases. Vet Rec 138(8), 175-7.

Sklaviadis, T., Akowitz, A., Manuelidis, E. E., and Manuelidis, L. (1993). Nucleic acid binding proteins in highly purified Creutzfeldt-Jakob disease preparations. Proc Natl Acad Sci U S A 90(12), 5713-7.

Solassol, J., Crozet, C., and Lehmann, S. (2003). Prion propagation in cultured cells. $\mathrm{Br}$ Med Bull 66, 87-97.

Soto, C. (2004). Diagnosing prion diseases: needs, challenges and hopes. Nat Rev Microbiol 2(10), 809-19.

Spraker, T. R., Miller, M. W., Williams, E. S., Getzy, D. M., Adrian, W. J., Schoonveld, G. G., Spowart, R. A., O'Rourke, K. I., Miller, J. M., and Merz, P. A. (1997). Spongiform encephalopathy in free-ranging mule deer (Odocoileus hemionus), white-tailed deer (Odocoileus virginianus) and Rocky Mountain elk (Cervus elaphus nelsoni) in northcentral Colorado. J Wildl Dis 33(1), 1-6.

Stahl, N., Baldwin, M. A., Teplow, D. B., Hood, L., Gibson, B. W., Burlingame, A. L., and Prusiner, S. B. (1993). Structural studies of the scrapie prion protein using mass spectrometry and amino acid sequencing. Biochemistry 32(8), 1991-2002.

Suter, S. E., Gouthro, T. A., McSweeney, P. A., Nash, R. A., Haskins, M. E., Felsburg, P. J., and Henthorn, P. S. (2006). Optimized transduction of canine paediatric CD34(+) cells using an MSCV-based bicistronic vector. Vet Res Commun 30(8), 881-901. 
Tamguney, G., Giles, K., Bouzamondo-Bernstein, E., Bosque, P. J., Miller, M. W., Safar, J., DeArmond, S. J., and Prusiner, S. B. (2006). Transmission of elk and deer prions to transgenic mice.J Virol 80(18), 9104-14.

Tang, Y., Thorne, J., Whatling, K., Jacobs, J. G., Langeveld, J., and Sauer, M. J. (2010). A single step multiplex immunofluorometric assay for differential diagnosis of BSE and scrapie. J Immunol Methods 356(1-2), 29-38.

Taraboulos, A., Raeber, A. J., Borchelt, D. R., Serban, D., and Prusiner, S. B. (1992). Synthesis and trafficking of prion proteins in cultured cells. Mol Biol Cell 3(8), 851-63.

Taraboulos, A., Rogers, M., Borchelt, D. R., McKinley, M. P., Scott, M., Serban, D., and Prusiner, S. B. (1990). Acquisition of protease resistance by prion proteins in scrapie-infected cells does not require asparagine-linked glycosylation. Proc Natl Acad Sci U S A 87(21), 8262-6.

Tateishi, J., Brown, P., Kitamoto, T., Hoque, Z. M., Roos, R., Wollman, R., Cervenakova, L., and Gajdusek, D. C. (1995). First experimental transmission of fatal familial insomnia. Nature 376(6539), 434-5.

Telling, G. C., Scott, M., Mastrianni, J., Gabizon, R., Torchia, M., Cohen, F. E., DeArmond, S. J., and Prusiner, S. B. (1995). Prion propagation in mice expressing human and chimeric PrP transgenes implicates the interaction of cellular PrP with another protein. Cell 83(1), 79-90.

Thackray, A. M., Hopkins, L., Klein, M. A., and Bujdoso, R. (2007). Mouse-adapted ovine scrapie prion strains are characterized by different conformers of PrPSc. J Virol 81(22), 12119-27.

Tobler, I., Gaus, S. E., Deboer, T., Achermann, P., Fischer, M., Rulicke, T., Moser, M., Oesch, B., McBride, P. A., and Manson, J. C. (1996). Altered circadian activity rhythms and sleep in mice devoid of prion protein. Nature 380(6575), 639-42.

Tremblay, P., Meiner, Z., Galou, M., Heinrich, C., Petromilli, C., Lisse, T., Cayetano, J., Torchia, M., Mobley, W., Bujard, H., DeArmond, S. J., and Prusiner, S. B. (1998). Doxycycline control of prion protein transgene expression modulates prion disease in mice. Proc Natl Acad Sci U S A 95(21), 12580-5.

Trifilo, M. J., Ying, G., Teng, C., and Oldstone, M. B. (2007). Chronic wasting disease of deer and elk in transgenic mice: oral transmission and pathobiology. Virology 365(1), 136-43.

Ukekawa, R., Miki, K., Fujii, M., Hirano, H., and Ayusawa, D. (2007). Accumulation of multiple forms of lamin A with down-regulation of FACE-1 suppresses growth in senescent human cells. Genes Cells 12(3), 397-406.

Vara, J., Perez-Gonzalez, J. A., and Jimenez, A. (1985). Biosynthesis of puromycin by Streptomyces alboniger: characterization of puromycin $\mathrm{N}$-acetyltransferase. Biochemistry 24(27), 8074-81.

Varmus, H. E., Padgett, T., Heasley, S., Simon, G., and Bishop, J. M. (1977). Cellular functions are required for the synthesis and integration of avian sarcoma virusspecific DNA. Cell 11(2), 307-19.

Viegas, P., Chaverot, N., Enslen, H., Perriere, N., Couraud, P. O., and Cazaubon, S. (2006). Junctional expression of the prion protein PrPC by brain endothelial cells: a role in trans-endothelial migration of human monocytes. J Cell Sci 119(Pt 22), 463443.

Vilette, D. (2008). Cell models of prion infection. Vet Res 39(4), 10.

Vilette, D., Andreoletti, O., Archer, F., Madelaine, M. F., Vilotte, J. L., Lehmann, S., and Laude, H. (2001). Ex vivo propagation of infectious sheep scrapie agent in 
heterologous epithelial cells expressing ovine prion protein. Proc Natl Acad Sci U $S$ A 98(7), 4055-9.

Vital, A., Fernagut, P. O., Canron, M. H., Joux, J., Bezard, E., Martin-Negrier, M. L., Vital, C., and Tison, F. (2009). The nigrostriatal pathway in Creutzfeldt-Jakob disease. $J$ Neuropathol Exp Neurol 68(7), 809-15.

Vital, A., Laplanche, J. L., Bastard, J. R., Xiao, X., Zou, W. Q., and Vital, C. (2011). A case of Gerstmann-Straussler-Scheinker disease with a novel six octapeptide repeat insertion. Neuropathol Appl Neurobiol 37(5), 554-9.

VLA (2011). Cattle BSE surveillance statistics. U.K. Veterinary Laboratories Agency.

Vorberg, I., and Priola, S. A. (2002). Molecular basis of scrapie strain glycoform variation. J Biol Chem 277(39), 36775-81.

Vorberg, I., Raines, A., and Priola, S. A. (2004). Acute formation of protease-resistant prion protein does not always lead to persistent scrapie infection in vitro. J Biol Chem 279(28), 29218-25.

Vorberg, I., Raines, A., Story, B., and Priola, S. A. (2004). Susceptibility of common fibroblast cell lines to transmissible spongiform encephalopathy agents. J Infect Dis 189(3), 431-9.

Wadsworth, J. D., Joiner, S., Linehan, J. M., Asante, E. A., Brandner, S., and Collinge, J. (2008). Review. The origin of the prion agent of kuru: molecular and biological strain typing. Philos Trans R Soc Lond B Biol Sci 363(1510), 3747-53.

Walsh, D. M., and Selkoe, D. J. (2004). Oligomers on the brain: the emerging role of soluble protein aggregates in neurodegeneration. Protein Pept Lett 11(3), 21328.

Ward, R. L., Porter, D. D., and Stevens, J. G. (1974). Nature of the scrapie agent: evidence against a viroid. J Virol 14(5), 1099-103.

Watt, N. T., and Hooper, N. M. (2003). The prion protein and neuronal zinc homeostasis. Trends Biochem Sci 28(8), 406-10.

Watzlawik, J., Skora, L., Frense, D., Griesinger, C., Zweckstetter, M., Schulz-Schaeffer, W. J., and Kramer, M. L. (2006). Prion protein helix1 promotes aggregation but is not converted into beta-sheet. J Biol Chem 281(40), 30242-50.

Weiss, E., Ramljak, S., Asif, A. R., Ciesielczyk, B., Schmitz, M., Gawinecka, J., SchulzSchaeffer, W., Behrens, C., and Zerr, I. (2010). Cellular prion protein overexpression disturbs cellular homeostasis in SH-SY5Y neuroblastoma cells but does not alter p53 expression: a proteomic study. Neuroscience 169(4), 1640-50.

Weissmann, C., Bueler, H., Fischer, M., Sailer, A., Aguzzi, A., and Aguet, M. (1994a). PrPdeficient mice are resistant to scrapie. Ann N Y Acad Sci 724, 235-40.

Weissmann, C., Bueler, H., Fischer, M., Sauer, A., and Aguet, M. (1994b). Susceptibility to scrapie in mice is dependent on PrPC. Philos Trans $R$ Soc Lond B Biol Sci 343(1306), 431-3.

Wells, G. A. (1993). Pathology of nonhuman spongiform encephalopathies: variations and their implications for pathogenesis. Dev Biol Stand 80, 61-9.

Wells, G. A., Scott, A. C., Johnson, C. T., Gunning, R. F., Hancock, R. D., Jeffrey, M., Dawson, M., and Bradley, R. (1987). A novel progressive spongiform encephalopathy in cattle. Vet Rec 121(18), 419-20.

Wemheuer, W. M., Benestad, S. L., Wrede, A., Schulze-Sturm, U., Wemheuer, W. E., Hahmann, U., Gawinecka, J., Schutz, E., Zerr, I., Brenig, B., Bratberg, B., Andreoletti, O., and Schulz-Schaeffer, W. J. (2009). Similarities between forms of sheep scrapie and Creutzfeldt-Jakob disease are encoded by distinct prion types. Am J Pathol 175(6), 2566-73. 
Wild, M. A., Spraker, T. R., Sigurdson, C. J., O'Rourke, K. I., and Miller, M. W. (2002). Preclinical diagnosis of chronic wasting disease in captive mule deer (Odocoileus hemionus) and white-tailed deer (Odocoileus virginianus) using tonsillar biopsy. J Gen Virol 83(Pt 10), 2629-34.

Wilesmith, J. W., Wells, G. A., Cranwell, M. P., and Ryan, J. B. (1988). Bovine spongiform encephalopathy: epidemiological studies. Vet Rec 123(25), 638-44.

Will, R. G., Ironside, J. W., Zeidler, M., Cousens, S. N., Estibeiro, K., Alperovitch, A., Poser, S., Pocchiari, M., Hofman, A., and Smith, P. G. (1996). A new variant of CreutzfeldtJakob disease in the UK. Lancet 347(9006), 921-5.

Williams, E. S. (2005). Chronic wasting disease. Vet Pathol 42(5), 530-49.

Williams, E. S., and Young, S. (1980). Chronic wasting disease of captive mule deer: a spongiform encephalopathy. J Wildl Dis 16(1), 89-98.

Williams, E. S., and Young, S. (1993). Neuropathology of chronic wasting disease of mule deer (Odocoileus hemionus) and elk (Cervus elaphus nelsoni). Vet Pathol 30(1), 36-45.

Windl, O., Buchholz, M., Neubauer, A., Schulz-Schaeffer, W., Groschup, M., Walter, S., Arendt, S., Neumann, M., Voss, A. K., and Kretzschmar, H. A. (2005). Breaking an absolute species barrier: transgenic mice expressing the mink PrP gene are susceptible to transmissible mink encephalopathy. J Virol 79(23), 14971-5.

Wolfe, L. L., Spraker, T. R., Gonzalez, L., Dagleish, M. P., Sirochman, T. M., Brown, J. C., Jeffrey, M., and Miller, M. W. (2007). PrPCWD in rectal lymphoid tissue of deer (Odocoileus spp.). J Gen Virol 88(Pt 7), 2078-82.

Wood, J. L., McGill, I. S., Done, S. H., and Bradley, R. (1997). Neuropathology of scrapie: a study of the distribution patterns of brain lesions in 222 cases of natural scrapie in sheep, 1982-1991. Vet Rec 140(7), 167-74.

Yadavalli, R., Guttmann, R. P., Seward, T., Centers, A. P., Williamson, R. A., and Telling, G. C. (2004). Calpain-dependent endoproteolytic cleavage of PrPSc modulates scrapie prion propagation. J Biol Chem 279(21), 21948-56.

Yang, Y., Vanin, E. F., Whitt, M. A., Fornerod, M., Zwart, R., Schneiderman, R. D., Grosveld, G., and Nienhuis, A. W. (1995). Inducible, high-level production of infectious murine leukemia retroviral vector particles pseudotyped with vesicular stomatitis virus G envelope protein. Hum Gene Ther 6(9), 1203-13.

Yee, J. K., Friedmann, T., and Burns, J. C. (1994). Generation of high-titer pseudotyped retroviral vectors with very broad host range. Methods Cell Biol 43 Pt A, 99-112.

Yokoyama, T., Masujin, K., Iwamaru, Y., Imamura, M., and Mohri, S. (2009). Alteration of the biological and biochemical characteristics of bovine spongiform encephalopathy prions during interspecies transmission in transgenic mice models. J Gen Virol 90(Pt 1), 261-8.

Zahn, R., Liu, A., Luhrs, T., Riek, R., von Schroetter, C., Lopez Garcia, F., Billeter, M., Calzolai, L., Wider, G., and Wuthrich, K. (2000). NMR solution structure of the human prion protein. Proc Natl Acad Sci US A 97(1), 145-50.

Zlotnik, I., and Rennie, J. C. (1962). The pathology of the brain of mice inoculated with tissues from scrapie sheep. J Comp Pathol 72, 360-5.

Zlotnik, I., and Rennie, J. C. (1965). Experimental Transmission of Mouse Passaged Scrapie to Goats, Sheep, Rats and Hamsters. J Comp Pathol 75, 147-57. 


\title{
7 Appendix
}

\subsection{PrP ORF of Saimiri sciureus}

GeneBank Submission ID: 1485013

\begin{abstract}
AtG GCG AAC CTT GGC TAC TGG ATG CTG GTT CTC TTT GTG GCC ACA TGG AGT GAC CTG GGC CTC TGT AAg AAg CGC CCG AAA CCT GGA GGA TGG AAT ACT GGG GGG AGC CGA TAC CCA GGC CAG GGC AGC CCT GGA GGC AAC CGC TAC CCA CCC CAG GGT GGT GGC TGG GGG CAG CCT CAT GGT GGT GGC TGG GGG CAA CCC CAT GGT GGC GGC TGG GGA CAG CCC CAT GGT GGC GGC TGG GGA CAG CCC CAT GGT GGT GGC TGG GGA CAG CCT CAT GGT GGC GGC TGG GGT CAA GGA GGT GGC ACC CAC AAT CAA TGG AAC AAG CCC AGT AAG CCA AAA ACC AAC ATG AAG CAC ATG GCT GGT GCT GCA GCG GCT GGG GCA GTG GTG GGG GGC CTT GGT GGC TAC ATG CTG GGA AGT GCC ATg AGC AGg CCC CTC ATA CAT TTT GGC AAT GAC TAT GAG GAC CGT TAC TAT CGT GAA AAC ATG TAC CGT TAC CCC AGC CAA GTA TAC TAC AAG CCC GTG GAT CAG TAC AGC AAC CAG AAC AAC TTT GTG CAC GAC TGC GTC AAC GTC ACG ATC AAG CAG CAC ACA GTC ACC ACC ACC ACC AAA GGG GAG AAC TTC ACT GAG ACC GAC GTG AAg ATg ATG GAg CGC GTg GTT GAg CAg ATg TGT ATC ACt CAg tAC GAG AAg GAA TCC CAG GCC TAT TAC CAG AgA GGA TCG AGC ATG GTC CTC TTC TCC TCC CCA CCT GTG ATC CTC CTG ATC TCT TTC CTC ATC TTC CTG ATA GTG GGA TGA
\end{abstract}

\subsection{Prion protein sequence of Saimiri sciureus}

MANLGYWMLVLFVATWSDLGLCKKRPKPGGWNTGGSRYPGQGSPGGNRYPPQGGGWGQPHGG GWGQPHGGGWGQPHGGGWGQPHGGGWGQPHGGGWGQGGGTHNQWNKPSKPKTNMKHMAGAAA AGAVVGGLGGYMLGSAMSRPLIHFGNDYEDRYYRENMYRYPSQVYYKPVDQYSNQNNFVHDC 
VNVTIKQHTVTTTTKGENFTETDVKMMERVVEQMCITQYEKESQAYYQRGSSMVLFSSPPVI LLISFLIFLIVG* 


\section{Curriculum Vitae}

Ann-Christin Schmädicke

born $2^{\text {nd }}$ January 1984 in Potsdam, Germany

Kreuzbergring 21, 37075 Göttingen

Email: schmaedicke@dpz.eu

Education

2008-present German Primate Center GmbH, Leibniz Institute for Primate Research, Göttingen, Unit of Infection Models, PhD thesis:

"Estimating Chronic wasting disease infectivity in cell culture"

2008-present Georg-August University, Göttingen, International PhD program "Molecular Medicine"

2007-2008 German Primate Center GmbH, Leibniz Institute for Primate Research, Göttingen, Dept. of Virology and Immunology, Master thesis: "Differential expression of microRNAs upon monkey pox infection"

2006-2008 Georg-August University, Göttingen, International Master program "Molecular Medicine",

2006 Institute for biomedical optics, University of Lübeck, Bachelor thesis: "Photodynamic therapy in combination with an antiseptic to kill gram-positive and gram-negative bacteria"

2003-2006 University of Lübeck, Bachelor program "Molecular life science"

2003

Sally-Bein Gymnasium, Beelitz, Abitur (A-level)

Additional activities

Student spokeperson for the PhD program "Molecular Medicine"

Membership in the examination board for the PhD program

"Molecular Medicine"

Publication Greenwood AD, Vincendeau M, Schmädicke AC, Montag J, Seifarth W and Motzkus D. "Bovine spongiform encephalopathy infection alters endogenous retrovirus expression in distinct brain regions of cynomolgus macaques (Macaca fascicularis)." Molecular Neurodegeneration, published June 23, 2011 


\section{Private Danksagung}

Ich möchte mich ganz herzlich bei allen Personen bedanken, die mich während meines Studiums und meiner Promotion unterstützt haben.

Lieber Dirk, diese Doktorarbeit war eine Zeit voller neuer Herausforderungen! Danke, dass du mich da sicher durchmanövriert hast.

Besonders bedanken möchte ich mich bei Sabine, du hast mir während meiner gesamten Zeit mit Rat und Tat zur Seite gestanden. Durch dich war ich immer in guter Gesellschaft, gemeinsam haben wir die Hochs und Tiefs der Prion Zellkultur durchgestanden. Du bist die Beste!

Meinen Mädels aus dem DPZ möchte ich auch herzlich danken. Liebe Aneela, Katharina R., Katharina T., Katrin, Kelly, Tina und vor allem Wiebke I. (du warst eine echt coole Wegbegleiterin), ihr wart immer da, wenn es mal schwierig war, mit Zuspruch, mit Ablenkung, mit Schokolade und Tee. Vor allem durch euch war mit einem zweiten Blick dann doch alles easy!

Ein riesiges Dankeschön geht an Christiane, Katherine, Kelly, Wiebke W. und Vanessa für eure Hilfe bei den Korrekturen, Formatierungen und für die kritische Auseinandersetzung mit meiner Arbeit.

Mein besonderer Dank gilt meinen Eltern. Lieber Papa, ich wünschte du könntest das hier noch alles miterleben; du hast mir meine wissenschaftliche Neugier und meinen Ehrgeiz mit auf den Weg gegeben. Liebe Mama, danke für deine Unterstützung und den Rückhalt während der letzten Jahre. Außerdem danke ich meiner gesamten Großfamilie für die Motivation in den letzten Jahren. Insbesondere bei Antje und Andi bedanke ich mich; euer kleines Paradies war gerade in der letzten Zeit ein wunderbarer Rückzugsort zum Erholen.

Mein größter Dank geht an alle Freunde, die mir in den letzten Jahren beigestanden haben. Und liebe Anna, liebe Wiebke, und liebe Van, die Zeit mit euch hier in Göttingen war toll! Ihr seit immer für mich da. Egal womit ich euch nerve, ich bin bei euch immer gut aufgehoben. Ihr seid meine emotionale Stütze! 